Supporting Information for

\title{
Mechanistic Study on Effect of Electron Donors in Propylene Polymerization using Ziegler-Natta Catalyst
}

Xing Guo, ${ }^{a}$ Liang Cui, ${ }^{b}$ Yisen Wang, ${ }^{b}$ Jianjun Yi, ${ }^{b}$ Jingwen Sun, ${ }^{a}$ Zhen Liu, $^{* a}$ Boping Liu ${ }^{* c}$ aSchool of Chemical Engineering, East China University of Science and Technology, Shanghai 200237, China

${ }^{b}$ Polyolefin Research Department, Petrochina Petrochemical Research Institute, Beijing 102206, China ${ }^{c}$ College of Materials and Energy, South China Agricultural University, Guangzhou 510642, China

*Corresponding author:

Zhen Liu, Email: liuzhen@ecust.edu.cn

Boping Liu, Email: boping@scau.edu.cn 


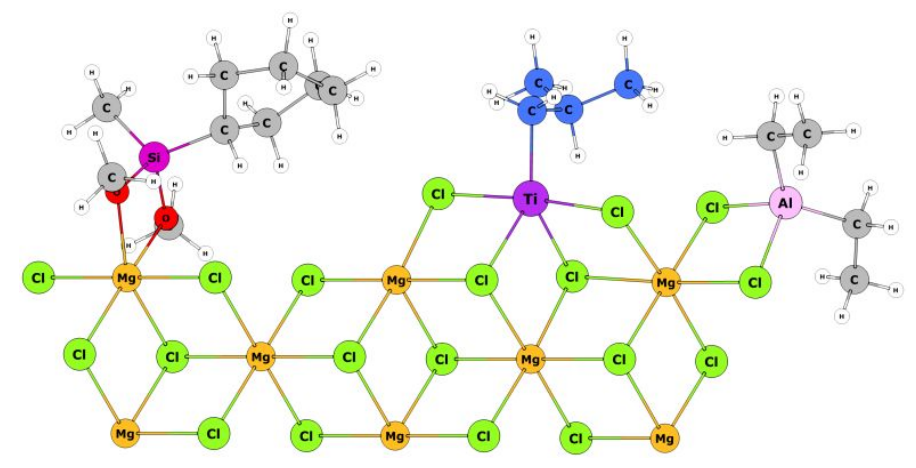

Figure S1. The Structure of the co-presence of CMDMS and $\mathrm{AlEt}_{2} \mathrm{Cl}$ around the Ti active site.

For the DiBS, CMDMS and DCPDMS studied here, the conformational analysis was performed using the Tinker program. The atoms were assigned the appropriate atom types based on the MMFF force field definition. All the initial structures obtained at the molecular mechanic (MM) level were then fully optimized using the Gaussian 09 program.

For the DiBS molecule, we found more than 20,000 conformers. Only the most stable one is given in the manuscript and supporting information. For the CMDMS and DCPDMS, we found 38 and 68 conformers, respectively. The energies of various conformational CMDMS and DCPDMS are listed in Table S1 and Table S2.

Table S1. Energies of various conformational CMDMS.

\begin{tabular}{cc}
\hline Conformer & Relative Energy $(\mathrm{kcal} / \mathrm{mol})$ \\
\hline CMDMS-1 & 0.000 \\
CMDMS-2 & 0.259 \\
CMDMS-3 & 0.264 \\
CMDMS-4 & 0.304 \\
CMDMS-5 & 0.353 \\
CMDMS-6 & 0.382 \\
CMDMS-7 & 0.750 \\
CMDMS-8 & 0.841 \\
CMDMS-9 & 0.921 \\
CMDMS-10 & 1.000 \\
CMDMS-11 & 1.025 \\
CMDMS-12 & 1.098 \\
CMDMS-13 & 1.139 \\
CMDMS-14 & 1.168 \\
CMDMS-15 & 1.175 \\
CMDMS-16 & 1.180 \\
CMDMS-17 & 1.282
\end{tabular}




\begin{tabular}{ll} 
CMDMS-18 & 1.287 \\
CMDMS-19 & 1.307 \\
CMDMS-20 & 1.347 \\
CMDMS-21 & 1.384 \\
CMDMS-22 & 1.395 \\
CMDMS-23 & 1.462 \\
CMDMS-24 & 1.510 \\
CMDMS-25 & 1.564 \\
CMDMS-26 & 1.579 \\
CMDMS-27 & 1.675 \\
CMDMS-28 & 1.842 \\
CMDMS-29 & 1.844 \\
CMDMS-30 & 1.940 \\
CMDMS-31 & 1.992 \\
CMDMS-32 & 2.369 \\
CMDMS-33 & 2.588 \\
CMDMS-34 & 2.609 \\
CMDMS-35 & 2.642 \\
CMDMS-36 & 3.193 \\
CMDMS-37 & 3.205 \\
CMDMS-38 & 3.286 \\
\hline
\end{tabular}

Table S2. Energies of various conformational DCPDMS.

\begin{tabular}{cc}
\hline Conformer & Relative Energy $(\mathrm{kcal} / \mathrm{mol})$ \\
\hline DCPDMS-1 & 0.000 \\
DCPDMS-2 & 0.031 \\
DCPDMS-3 & 0.153 \\
DCPDMS-4 & 0.287 \\
DCPDMS-5 & 0.740 \\
DCPDMS-6 & 0.749 \\
DCPDMS-7 & 0.848 \\
DCPDMS-8 & 0.848 \\
DCPDMS-9 & 0.923 \\
DCPDMS-10 & 1.025 \\
DCPDMS-11 & 1.233 \\
DCPDMS-12 & 1.256 \\
DCPDMS-13 & 1.350 \\
DCPDMS-14 & 1.362 \\
DCPDMS-15 & 1.407 \\
DCPDMS-16 & 1.485 \\
DCPDMS-17 & 1.488 \\
DCPDMS-18 & 1.492
\end{tabular}




\begin{tabular}{|c|c|}
\hline DCPDMS-19 & 1.504 \\
\hline DCPDMS-20 & 1.551 \\
\hline DCPDMS-21 & 1.610 \\
\hline DCPDMS-22 & 1.652 \\
\hline DCPDMS-23 & 1.707 \\
\hline DCPDMS-24 & 1.758 \\
\hline DCPDMS-25 & 1.780 \\
\hline DCPDMS-26 & 1.794 \\
\hline DCPDMS-27 & 1.829 \\
\hline DCPDMS-28 & 1.864 \\
\hline DCPDMS-29 & 1.884 \\
\hline DCPDMS-30 & 1.897 \\
\hline DCPDMS-31 & 1.915 \\
\hline DCPDMS-32 & 1.936 \\
\hline DCPDMS-33 & 1.944 \\
\hline DCPDMS-34 & 1.993 \\
\hline DCPDMS-35 & 2.161 \\
\hline DCPDMS-36 & 2.242 \\
\hline DCPDMS-37 & 2.255 \\
\hline DCPDMS-38 & 2.262 \\
\hline DCPDMS-39 & 2.293 \\
\hline DCPDMS-40 & 2.368 \\
\hline DCPDMS-41 & 2.420 \\
\hline DCPDMS-42 & 2.423 \\
\hline DCPDMS-43 & 2.468 \\
\hline DCPDMS-44 & 2.470 \\
\hline DCPDMS-45 & 2.474 \\
\hline DCPDMS-46 & 2.488 \\
\hline DCPDMS-47 & 2.826 \\
\hline DCPDMS-48 & 2.876 \\
\hline DCPDMS-49 & 3.072 \\
\hline DCPDMS-50 & 3.113 \\
\hline DCPDMS-51 & 3.205 \\
\hline DCPDMS-52 & 3.278 \\
\hline DCPDMS-53 & 3.281 \\
\hline DCPDMS-54 & 3.288 \\
\hline DCPDMS-55 & 3.494 \\
\hline DCPDMS-56 & 3.522 \\
\hline DCPDMS-57 & 3.572 \\
\hline DCPDMS-58 & 3.587 \\
\hline DCPDMS-59 & 3.711 \\
\hline DCPDMS-60 & 3.742 \\
\hline DCPDMS-61 & 3.761 \\
\hline DCPDMS-62 & 3.814 \\
\hline
\end{tabular}




\begin{tabular}{ll} 
DCPDMS-63 & 3.908 \\
DCPDMS-64 & 4.179 \\
DCPDMS-65 & 4.307 \\
DCPDMS-66 & 4.588 \\
DCPDMS-67 & 5.621 \\
DCPDMS-68 & 5.948 \\
\hline
\end{tabular}

List of Cartesian coordinates

Propylene
$\begin{array}{lrrr}\text { C } & -1.28396100 & -0.22595400 & 0.00000200 \\ \text { H } & -1.29674100 & -1.32226700 & -0.00002100 \\ \text { H } & -2.25434700 & 0.27882400 & 0.00003400 \\ \text { C } & -0.13791000 & 0.46083000 & -0.00001300 \\ \text { H } & -0.18779000 & 1.55735700 & -0.00000200 \\ \text { C } & 1.24077400 & -0.15689900 & -0.00000100 \\ \text { H } & 2.03230100 & 0.60749900 & -0.00098600 \\ \text { H } & 1.39692500 & -0.79383400 & 0.88753800 \\ \text { H } & 1.39623500 & -0.79544700 & -0.88649600\end{array}$

Ethyl benzoate (EB)

$\begin{array}{lrrr}\text { C } & -3.34809200 & -0.65301900 & 0.00000000 \\ \text { C } & -3.06252500 & 0.71741900 & 0.00000800 \\ \text { C } & -1.73733400 & 1.15293800 & -0.00000400 \\ \text { C } & -0.68878000 & 0.21949200 & -0.00002500 \\ \text { C } & -0.97801300 & -1.15455000 & -0.00003400 \\ \text { C } & -2.30577300 & -1.58668700 & -0.00002100 \\ \text { H } & -2.52952700 & -2.65642600 & -0.00002800 \\ \text { H } & -4.38657200 & -0.99456700 & 0.00001000 \\ \text { H } & -3.87651400 & 1.44658100 & 0.00002500 \\ \text { H } & -1.48775300 & 2.21578300 & 0.00000300 \\ \text { H } & -0.15822700 & -1.87389700 & -0.00005000 \\ \text { C } & 0.71116000 & 0.74219400 & -0.00004000 \\ \text { O } & 1.63080800 & -0.24141100 & -0.00001300 \\ \text { C } & 3.00841900 & 0.16484200 & 0.00002900 \\ \text { C } & 3.85879800 & -1.08814700 & 0.00005600 \\ \text { H } & 3.19667500 & 0.79522900 & -0.88494500 \\ \text { H } & 3.19662200 & 0.79523100 & 0.88501300 \\ \text { H } & 3.65364200 & -1.69974300 & -0.89213000 \\ \text { H } & 3.65358900 & -1.69974100 & 0.89223200 \\ \text { H } & 4.92690500 & -0.82086200 & 0.00008800 \\ \text { O } & 1.00219300 & 1.91710000 & 0.00000900\end{array}$


Diethyl-2,3-diisobutylsuccinate (DiBS)

\begin{tabular}{|c|c|c|c|}
\hline$C$ & -0.68116700 & -0.39057500 & -0.82917000 \\
\hline $\mathrm{H}$ & -0.59273300 & -1.14804500 & -1.62157000 \\
\hline & 0.68121000 & 0.39039200 & -0.82921000 \\
\hline & 0.59282700 & 1.14769700 & -1.62177300 \\
\hline & -0.85170900 & -1.12649400 & 0.49180300 \\
\hline & 0.85167400 & 1.12658900 & 0.49161900 \\
\hline & 0.28011300 & 2.34202700 & 0.46623500 \\
\hline & -0.28010000 & -2.34191600 & 0.46672600 \\
\hline C & 0.18749700 & 3.05753300 & 1.71701100 \\
\hline & 1.09564800 & 2.86438700 & 2.30702900 \\
\hline & 0.16155100 & 4.11969200 & 1.43331500 \\
\hline C & -1.06196300 & 2.64151700 & 2.47552000 \\
\hline $\mathrm{H}$ & -1.96779300 & 2.87534600 & 1.89458100 \\
\hline H & -1.05210100 & 1.55935300 & 2.66906300 \\
\hline & -1.11644200 & 3.17857600 & 3.43602800 \\
\hline C & -0.18755600 & -3.05714300 & 1.71766600 \\
\hline $\mathrm{H}$ & -1.09575700 & -2.86389400 & 2.30757400 \\
\hline $\mathrm{H}$ & -0.16155700 & -4.11936400 & 1.43420800 \\
\hline C & 1.06183500 & -2.64091700 & 2.47617300 \\
\hline $\mathrm{H}$ & 1.11626000 & -3.17775400 & 3.43680900 \\
\hline $\mathrm{H}$ & 1.96771600 & -2.87485100 & 1.89535500 \\
\hline $\mathrm{H}$ & 1.05192500 & -1.55870900 & 2.66946700 \\
\hline & -1.40490600 & -0.66060400 & 1.46139400 \\
\hline 0 & 1.40475600 & 0.66087600 & 1.46136200 \\
\hline C & -1.87836600 & 0.52807600 & -1.11289400 \\
\hline 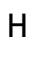 & -1.70864300 & 1.00167800 & -2.09696500 \\
\hline$H$ & -1.88069600 & 1.34190800 & -0.37308600 \\
\hline C & -3.26044400 & -0.14967100 & -1.11713800 \\
\hline $\mathrm{H}$ & -3.45092500 & -0.51731800 & -0.09412500 \\
\hline C & -4.34384800 & 0.88389300 & -1.44772400 \\
\hline $\mathrm{H}$ & -5.35120100 & 0.44067900 & -1.39134300 \\
\hline $\mathrm{H}$ & -4.31048200 & 1.73638600 & -0.74990100 \\
\hline & -4.21113000 & 1.28283300 & -2.46866400 \\
\hline 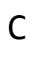 & -3.33116300 & -1.34533700 & -2.07555000 \\
\hline $\mathrm{H}$ & -2.64830700 & -2.15729800 & -1.77886300 \\
\hline $\mathrm{H}$ & -4.34799000 & -1.76925400 & -2.10253100 \\
\hline n & -3.06899000 & -1.04314400 & -3.10493000 \\
\hline C & 1.87842300 & -0.52832200 & -1.11266800 \\
\hline $\mathrm{H}$ & 1.88071100 & -1.34199500 & -0.37268500 \\
\hline H & 1.70875400 & -1.00213500 & -2.09664700 \\
\hline C & 3.26050400 & 0.14942000 & -1.11698200 \\
\hline & 3.45092600 & 0.51729300 & -0.0940400 \\
\hline
\end{tabular}




$\begin{array}{lrrr}\mathrm{C} & 3.33128000 & 1.34487300 & -2.07565600 \\ \mathrm{H} & 4.34811000 & 1.76878100 & -2.10267200 \\ \mathrm{H} & 3.06916500 & 1.04245200 & -3.10498300 \\ \mathrm{H} & 2.64841000 & 2.15690200 & -1.77918800 \\ \mathrm{C} & 4.34392400 & -0.88422000 & -1.44727700 \\ \mathrm{H} & 4.21126400 & -1.28338700 & -2.46813600 \\ \mathrm{H} & 5.35127400 & -0.44099700 & -1.39093600 \\ \mathrm{H} & 4.31051500 & -1.73655800 & -0.74926700\end{array}$

Cyclohexylmethyldimethoxysilane (CMDMS)

$\begin{array}{lrrr}\text { C } & -0.69569800 & -0.00119200 & -0.18066700 \\ \text { C } & -1.34585400 & -1.40776100 & -0.28481600 \\ \text { C } & -1.53576300 & 0.91235400 & 0.73446900 \\ \text { H } & -0.69762600 & 0.45243200 & -1.19046700 \\ \text { C } & -2.88634200 & -1.35263800 & -0.15483300 \\ \text { H } & -0.94642700 & -2.08226900 & 0.49343300 \\ \text { H } & -1.05755700 & -1.85932400 & -1.24755200 \\ \text { C } & -2.93983700 & 1.16629300 & 0.13837400 \\ \text { H } & -1.63184100 & 0.42850400 & 1.72292000 \\ \text { H } & -1.02243800 & 1.87044900 & 0.91994600 \\ \text { C } & -3.44073100 & -0.03210900 & -0.69920500 \\ \text { H } & -3.17807900 & -1.44781500 & 0.90623700 \\ \text { H } & -3.34055900 & -2.21324700 & -0.67267900 \\ \text { H } & -2.91936400 & 2.07136300 & -0.49227500 \\ \text { H } & -3.65014000 & 1.37813300 & 0.95577000 \\ \text { H } & -3.11536400 & 0.08070900 & -1.74850200 \\ \text { H } & -4.54259800 & -0.04790400 & -0.72291600 \\ \text { C } & 1.42724300 & -0.71691700 & 2.03686300 \\ \text { H } & 2.49871600 & -0.66242600 & 2.28722000 \\ \text { H } & 0.87866600 & -0.12333400 & 2.78548600 \\ \text { H } & 1.10533800 & -1.76670900 & 2.12508100 \\ \text { O } & 1.85284600 & -0.99988000 & -0.85153300 \\ \text { O } & 1.77559500 & 1.48068200 & 0.30874800 \\ \text { C } & 3.21530000 & -1.35875200 & -0.85583600 \\ \text { H } & 3.44921200 & -1.86482200 & -1.80688500 \\ \text { H } & 3.87942600 & -0.47822900 & -0.76458300 \\ \text { H } & 3.46334200 & -2.05624300 & -0.03240600 \\ \text { C } & 1.71842300 & 2.36163800 & -0.78998000 \\ \text { H } & 2.05556800 & 1.88252600 & -1.72870600 \\ \text { H } & 0.69433400 & 2.74865600 & -0.95619200 \\ \text { H } & 2.37584000 & 3.22418800 & -0.59195700 \\ & 1.11239900 & -0.05761100 & 0.31679300\end{array}$

Dicyclopentyldimethoxysilane (DCPDMS) 


\begin{tabular}{lrrr} 
C & 1.50996200 & -0.57673900 & -0.42289200 \\
C & 2.83015900 & 0.23328400 & -0.62347500 \\
C & 1.86447900 & -1.62702000 & 0.65808500 \\
H & 1.28243400 & -1.12273000 & -1.35657500 \\
C & 3.95750500 & -0.61200100 & 0.03682400 \\
H & 2.77322100 & 1.22473900 & -0.14847400 \\
H & 3.02049800 & 0.40442200 & -1.69473400 \\
C & 3.32304100 & -1.97990200 & 0.33964400 \\
H & 1.18871300 & -2.49762400 & 0.64750000 \\
H & 1.80577700 & -1.17701100 & 1.66548700 \\
H & 4.85610300 & -0.69021900 & -0.59496200 \\
H & 4.27174800 & -0.13880500 & 0.98170000 \\
H & 3.35564300 & -2.62451800 & -0.55674000 \\
H & 3.83211000 & -2.52228000 & 1.15276000 \\
C & -1.50996200 & -0.57673900 & 0.42289200 \\
C & -2.83015900 & 0.23328500 & 0.62347500 \\
C & -1.86447900 & -1.62701900 & -0.65808500 \\
H & -1.28243400 & -1.12273000 & 1.35657500 \\
C & -3.95750500 & -0.61200100 & -0.03682300 \\
H & -2.77322100 & 1.22473900 & 0.14847300 \\
H & -3.02049800 & 0.40442200 & 1.69473400 \\
C & -3.32304100 & -1.97990200 & -0.33964400 \\
H & -1.18871300 & -2.49762400 & -0.64750000 \\
H & -1.80577800 & -1.17701000 & -1.66548700 \\
H & -4.85610300 & -0.69021900 & 0.59496300 \\
H & -4.27174900 & -0.13880500 & -0.98169900 \\
H & -3.35564300 & -2.62451800 & 0.55674000 \\
H & -3.83211000 & -2.52228000 & -1.15276000 \\
O & 0.50624400 & 1.41557700 & 1.27136200 \\
O & -0.50624400 & 1.41557700 & -1.27136200 \\
C & 0.28298900 & 2.35969900 & -1.95630900 \\
H & 1.05053100 & 1.87607700 & -2.59011500 \\
H & -0.36744600 & 2.96088800 & -2.61299000 \\
H & 0.80333700 & 3.04977800 & -1.26538700 \\
H & -0.28298800 & 2.35970000 & 1.95630900 \\
\hline & 0.36744700 & 2.96088800 & 2.61299000 \\
-0.80333600 & 3.04977800 & 1.26538700 \\
H & 0.00000000 & 0.44981800 & 0.00000000
\end{tabular}

AlEt ${ }_{2}$ Cl
$\begin{array}{lllr}\text { C } & -2.03478000 & -0.56232700 & -0.01845600 \\ \text { H } & -2.22990300 & -1.24441200 & 0.83133800 \\ \text { H } & -2.22738900 & -1.18530600 & -0.91346500\end{array}$




$\begin{array}{lrrr}\text { C } & 0.63094900 & 1.67778900 & -0.00931900 \\ \text { H } & 0.20273300 & 2.19793700 & -0.88957700 \\ \text { H } & 0.18520600 & 2.20934400 & 0.85518500 \\ \text { C } & 2.16159500 & 1.81900700 & 0.00485500 \\ \text { H } & 2.48053900 & 2.87546500 & 0.00013200 \\ \text { H } & 2.60557100 & 1.34620100 & 0.89614200 \\ \text { H } & 2.62327600 & 1.33284500 & -0.87008600 \\ \text { C } & -3.00335600 & 0.63348600 & 0.01911100 \\ \text { H } & -2.85962400 & 1.30728300 & -0.84263200 \\ \text { H } & -4.05937700 & 0.31346500 & 0.00389100 \\ \text { H } & -2.86653300 & 1.24550400 & 0.92682700 \\ \text { Cl } & 1.24551300 & -1.76768800 & 0.00627600 \\ \text { Al } & -0.11959000 & -0.13502800 & -0.00627600\end{array}$

$\mathrm{MgCl}_{2}$-Ti-isobutyl

$\begin{array}{lrrr}\text { Cl } & 8.08445300 & -0.06746000 & -1.42821200 \\ \text { Mg } & 7.30372700 & -1.82182800 & 0.18129800 \\ \text { Cl } & 8.85282500 & 2.23251800 & 1.01529800 \\ \text { Mg } & 6.77693700 & 1.74837000 & -0.30146000 \\ \text { Cl } & 5.22784100 & -2.30597700 & -1.13546000 \\ \text { Cl } & 1.84445100 & -0.97427800 & -1.32547700 \\ \text { Cl } & 4.70105100 & 1.26422300 & -1.61821900 \\ \text { Cl } & 3.13960000 & -2.24451300 & 1.60080300 \\ \text { Mg } & 1.06373000 & -2.72865400 & 0.28405100 \\ \text { Cl } & 5.99621200 & -0.00599700 & 1.30805100 \\ \text { Mg } & 3.92033500 & -0.49012200 & -0.00873300 \\ \text { Cl } & 2.61280300 & 1.32567800 & 1.11805700 \\ \text { Mg } & 0.53661700 & 0.84135100 & -0.19885100 \\ \text { Cl } & -1.01210300 & -3.21275200 & -1.03272600 \\ \text { Cl } & -4.39556900 & -1.88108300 & -1.22281400 \\ \text { Cl } & -1.54681900 & 0.43878400 & -1.61752700 \\ \text { Cl } & -3.10052000 & -3.15136100 & 1.70348100 \\ \text { Mg } & -5.17630000 & -3.63550000 & 0.38678200 \\ \text { Cl } & -0.24383000 & -0.91279200 & 1.41087800 \\ \text { Mg } & -2.31960400 & -1.39697000 & 0.09423200 \\ \text { Cl } & -3.59141900 & 0.52839000 & 1.28150400 \\ \text { Mg } & -5.70282600 & -0.06528800 & -0.09595300 \\ \text { Cl } & -7.77898600 & -0.54944900 & -1.41271300 \\ \text { Cl } & -6.48382700 & -1.81964500 & 1.51355600 \\ \text { Ti } & -2.83608700 & 2.32863900 & -0.43578400 \\ \text { Cl } & -4.92552800 & 1.76839000 & -1.53967900 \\ \text { Cl } & -0.63956400 & 2.79598300 & 0.57886700 \\ \text { C } & -3.16656900 & 4.34975900 & -0.72352200 \\ \text { C } & -3.85800400 & 4.34755300 & 0.62600700\end{array}$




$\begin{array}{lrrr}\text { H } & -2.23459200 & 4.92992500 & -0.77138600 \\ \text { H } & -3.82339900 & 4.54536700 & -1.58255400 \\ \text { H } & -3.83051500 & 3.27519300 & 1.04665100 \\ \text { C } & -3.13283400 & 5.17445100 & 1.69694500 \\ \text { H } & -3.19678000 & 6.24298500 & 1.43737500 \\ \text { H } & -3.59312200 & 5.03348300 & 2.68754400 \\ \text { H } & -2.07119000 & 4.89901300 & 1.76615000 \\ \text { C } & -5.35722000 & 4.67042800 & 0.55615300 \\ \text { H } & -5.86583300 & 4.04261800 & -0.18877900 \\ \text { H } & -5.84314100 & 4.51925700 & 1.53328600 \\ \text { H } & -5.48934200 & 5.72545700 & 0.26866000\end{array}$

$\begin{array}{lrrr}\text { Donor } & \text { Coordination } & & \\ \text { MgCl }{ }_{2}-\text { Ti-isobutyl-EB-Mg1 } & \\ \text { Cl } & -7.65993900 & -1.28569900 & 0.94229800 \\ \text { Mg } & -6.63052000 & -3.10091200 & -0.44448300 \\ \text { Cl } & -8.53719200 & 0.66458200 & -1.75545400 \\ \text { Mg } & -6.48883300 & 0.53514700 & -0.31843200 \\ \text { Cl } & -4.58216200 & -3.23034800 & 0.99253800 \\ \text { Cl } & -1.36267900 & -1.53894300 & 1.16883700 \\ \text { Cl } & -4.44047500 & 0.40571100 & 1.11858900 \\ \text { Cl } & -2.38163900 & -3.22471400 & -1.65497700 \\ \text { Mg } & -0.33333400 & -3.35414400 & -0.21799600 \\ \text { Cl } & -5.45941600 & -1.28006600 & -1.70521500 \\ \text { Mg } & -3.41106200 & -1.40949200 & -0.26818500 \\ \text { Cl } & -2.23996800 & 0.41133300 & -1.52890300 \\ \text { Mg } & -0.19124400 & 0.28189200 & -0.09179900 \\ \text { Cl } & 1.71500100 & -3.48353600 & 1.21906700 \\ \text { Cl } & 4.93453600 & -1.79212100 & 1.39545400 \\ \text { Cl } & 1.97126200 & 0.27192300 & 1.33447200 \\ \text { Cl } & 3.91570600 & -3.47794200 & -1.42838200 \\ \text { Mg } & 5.96395600 & -3.60739200 & 0.00857900 \\ \text { Cl } & 0.83787500 & -1.53329900 & -1.47870100 \\ \text { Mg } & 2.88613400 & -1.66285200 & -0.04184300 \\ \text { Cl } & 4.13163300 & 0.16818900 & -1.47058100 \\ \text { Mg } & 6.10530300 & 0.02866900 & 0.13458500 \\ \text { Cl } & 8.15401600 & -0.10077300 & 1.57163200 \\ \text { Cl } & 7.13508700 & -1.78652000 & -1.25216800 \\ \text { Ti } & 3.02785800 & 2.07734400 & -0.13389000 \\ \text { Cl } & 5.09229000 & 1.99792200 & 1.18552800 \\ \text { Cl } & 1.05290400 & 1.92029600 & -1.52927300 \\ \text { C } & 3.00821300 & 4.14455900 & -0.12094100 \\ \text { C } & 3.82695900 & 4.09199000 & -1.39899700 \\ \text { H } & 1.99336400 & 4.55210600 & -0.23473200\end{array}$




$\begin{array}{lrrr}\text { H } & 3.53067600 & 4.57076100 & 0.74737000 \\ \text { H } & 4.00654800 & 2.98942600 & -1.67256900 \\ \text { C } & -3.89627100 & 5.15657300 & -0.89251000 \\ \text { C } & -2.70713400 & 4.56570800 & -1.33131900 \\ \text { C } & -2.02612400 & 3.67003200 & -0.50688900 \\ \text { C } & -2.53355400 & 3.37039800 & 0.76677000 \\ \text { C } & -3.72424600 & 3.97293900 & 1.21222000 \\ \text { C } & -4.40378600 & 4.86104500 & 0.38053200 \\ \text { H } & -5.33241600 & 5.32561600 & 0.72098900 \\ \text { H } & -4.43352600 & 5.85055500 & -1.54416700 \\ \text { H } & -2.31314400 & 4.79214700 & -2.32444200 \\ \text { H } & -1.10762900 & 3.19387000 & -0.84636700 \\ \text { H } & -4.11459000 & 3.72206600 & 2.19878800 \\ \text { C } & -1.83358100 & 2.37525500 & 1.60261300 \\ \text { O } & -2.27381800 & 2.28873700 & 2.83785100 \\ \text { C } & -1.76134800 & 1.22635800 & 3.68149200 \\ \text { C } & -2.55991300 & 1.24721600 & 4.96463600 \\ \text { H } & -1.87283200 & 0.27630100 & 3.14079200 \\ \text { H } & -0.68552900 & 1.40065200 & 3.84207800 \\ \text { H } & -3.62508300 & 1.06862000 & 4.75350600 \\ \text { H } & -2.45841000 & 2.21401300 & 5.48098100 \\ \text { H } & -2.20033700 & 0.45343800 & 5.63703600 \\ \text { O } & -0.90144300 & 1.66742400 & 1.20259500 \\ \text { C } & 5.24223500 & 4.66596600 & -1.24900200 \\ \text { H } & 5.84198400 & 4.48862200 & -2.15599100 \\ \text { H } & 5.18138800 & 5.75365500 & -1.08550800 \\ \text { H } & 5.76220100 & 4.21426600 & -0.39288900 \\ \text { C } & 3.09592700 & 4.65661600 & -2.62462200 \\ \text { H } & 2.96618900 & 5.74389400 & -2.50308400 \\ \text { H } & 3.67090000 & 4.48017100 & -3.54749300 \\ \text { H } & 2.10413700 & 4.19811900 & -2.74226400\end{array}$

$\mathrm{MgCl}_{2}$-Ti-i sobutyl-EB-Mg2

$\begin{array}{lrrr}\text { Cl } & 7.24347800 & -1.86824400 & -1.78020900 \\ \text { Mg } & 6.30516700 & -3.37647700 & -0.01305000 \\ \text { Cl } & 8.42567200 & 0.47357500 & 0.44895600 \\ \text { Mg } & 6.24617200 & 0.18790300 & -0.75357600 \\ \text { Cl } & 4.12573400 & -3.66212900 & -1.21553600 \\ \text { Cl } & 0.94907100 & -1.89158000 & -1.39139000 \\ \text { Cl } & 4.06680600 & -0.09771500 & -1.95607200 \\ \text { Cl } & 2.19025800 & -3.11412700 & 1.57825800 \\ \text { Mg } & 0.01087600 & -3.39977100 & 0.37580400 \\ \text { Cl } & 5.30801200 & -1.32025600 & 1.01363100 \\ \text { Mg } & 3.12851900 & -1.60589400 & -0.18888800\end{array}$




\begin{tabular}{|c|c|c|c|}
\hline $\mathrm{Cl}$ & 2.13133000 & 0.45027500 & 0.83772800 \\
\hline Mg & -0.04852800 & 0.16463700 & -0.36483900 \\
\hline $\mathrm{Cl}$ & -2.16854200 & -3.68538700 & -0.82674600 \\
\hline $\mathrm{Cl}$ & -5.34527700 & -1.91484000 & -1.0026910 \\
\hline $\mathrm{Cl}$ & -2.22892800 & -0.02812700 & -1.6733780 \\
\hline $\mathrm{Cl}$ & -4.10421400 & -3.13742500 & 1.9670130 \\
\hline Mg & -6.28354300 & -3.42309300 & 0.76456706 \\
\hline $\mathrm{Cl}$ & -0.98644900 & -1.34354800 & 1.4024310 \\
\hline Mg & -3.16578000 & -1.62937400 & 0.20008606 \\
\hline $\mathrm{Cl}$ & -4.11946500 & 0.53512100 & 1.28639406 \\
\hline Mg & -6.34207100 & 0.14133400 & 0.02409906 \\
\hline $\mathrm{Cl}$ & -8.52193400 & -0.14433000 & -1.1784170 \\
\hline $\mathrm{Cl}$ & -7.28077800 & -1.36685300 & 1.79122306 \\
\hline $\mathrm{Ti}$ & -3.18059500 & 2.08416300 & -0.5790930 \\
\hline $\mathrm{Cl}$ & -5.38331600 & 1.76734100 & $-1.5621760 e$ \\
\hline $\mathrm{Cl}$ & -0.93368100 & 2.27798500 & 0.3889910 \\
\hline C & -3.18847200 & 4.09896500 & $-1.0468400 €$ \\
\hline C & -3.80628800 & 4.33580000 & 0.31828906 \\
\hline $\mathrm{H}$ & -2.17304300 & 4.49975200 & -1.17584006 \\
\hline $\mathrm{H}$ & -3.83960400 & 4.33326400 & -1.90062300 \\
\hline $\mathrm{H}$ & -3.92285900 & 3.31880200 & $0.8472360 e$ \\
\hline C & 1.30364600 & 4.65483200 & -1.30291800 \\
\hline C & 2.16962200 & 3.90974400 & -2.11091006 \\
\hline C & 3.29154000 & 3.29562900 & -1.55245900 \\
\hline C & 3.54814500 & 3.42679000 & -0.17872400 \\
\hline C & 2.67735700 & 4.17698300 & 0.63029506 \\
\hline C & 1.55962300 & 4.78907500 & 0.06664400 \\
\hline $\mathrm{H}$ & 0.87481100 & 5.35998600 & 0.69777500 \\
\hline $\mathrm{H}$ & 0.42046500 & 5.12861700 & -1.74021000 \\
\hline $\mathrm{H}$ & 1.96803500 & 3.80175700 & -3.17942400 \\
\hline $\mathrm{H}$ & 3.95631900 & 2.68400800 & -2.16128300 \\
\hline $\mathrm{H}$ & 2.87384200 & 4.25436000 & 1.69977100 \\
\hline C & 4.70164300 & 2.70233300 & 0.40547000 \\
\hline 0 & 4.83783000 & 2.85353700 & 1.70297300 \\
\hline C & 5.87722900 & 2.10673100 & 2.39715800 \\
\hline C & 6.03904800 & 2.72025900 & 3.76869400 \\
\hline $\mathrm{H}$ & 5.54194200 & 1.05982900 & 2.44916400 \\
\hline $\mathrm{H}$ & 6.80410000 & 2.12289600 & 1.80454100 \\
\hline $\mathrm{H}$ & 5.09705200 & 2.68244300 & 4.33747700 \\
\hline $\mathrm{H}$ & 6.36163600 & 3.77026900 & 3.69392100 \\
\hline $\mathrm{H}$ & 6.80709000 & 2.16310700 & 4.32658500 \\
\hline 0 & 5.46945000 & 2.00698400 & -0.26615300 \\
\hline C & -5.23942500 & 4.88235300 & 0.26240000 \\
\hline $\mathrm{H}$ & -5.69483100 & 4.90912100 & 1.2651110 \\
\hline
\end{tabular}




$\begin{array}{llll}\text { H } & -5.22384900 & 5.90980500 & -0.13414400 \\ \text { H } & -5.87173100 & 4.26717600 & -0.39305400 \\ \text { C } & -2.91088200 & 5.13910200 & 1.27228600 \\ \text { H } & -2.82379500 & 6.17376100 & 0.90436600 \\ \text { H } & -3.33703200 & 5.17062900 & 2.28735100 \\ \text { H } & -1.90236000 & 4.70635700 & 1.33377800\end{array}$

$\mathrm{MgCl}_{2}$-Ti-isobutyl-EB-Ti

\begin{tabular}{lrrr} 
Cl & -8.44344100 & 0.19119300 & 1.49525400 \\
Mg & -7.80719000 & -1.92585100 & 0.31577200 \\
Cl & -9.29540900 & 1.87189600 & -1.38579000 \\
Mg & -7.15801700 & 1.63253800 & -0.10055800 \\
Cl & -5.66979900 & -2.16520900 & 1.60100400 \\
Cl & -2.24695900 & -0.96323000 & 1.29043600 \\
Cl & -5.02062600 & 1.39317900 & 1.18467600 \\
Cl & -3.74812700 & -2.84090500 & -1.17429200 \\
Mg & -1.61078100 & -3.08025700 & 0.11091100 \\
Cl & -6.52176800 & -0.48450400 & -1.28004200 \\
Mg & -4.38438000 & -0.72385000 & 0.00520100 \\
Cl & -3.09897400 & 0.71747900 & -1.59058600 \\
Mg & -0.96131200 & 0.47806700 & -0.30537300 \\
Cl & 0.52658800 & -3.31956600 & 1.39617200 \\
Cl & 3.94947600 & -2.11759100 & 1.08564600 \\
Cl & 1.10376700 & 0.15367400 & 1.08502000 \\
Cl & 2.44843100 & -3.99531500 & -1.37907100 \\
Mg & 4.58570500 & -4.23467700 & -0.09389300 \\
Cl & -0.32527200 & -1.63893000 & -1.48487900 \\
Mg & 1.81201000 & -1.87834000 & -0.19974500 \\
Cl & 2.99691100 & -0.42147500 & -1.83562300 \\
Mg & 5.23466300 & -0.67625800 & -0.51017700 \\
Cl & 7.37230000 & -0.91565900 & 0.77498800 \\
Cl & 5.87115000 & -2.79331500 & -1.68972500 \\
Ti & 2.74744200 & 2.09332900 & -1.11472900 \\
Cl & 5.21969500 & 1.73755500 & -0.92103200 \\
Cl & 0.22831200 & 2.31673400 & -1.36040900 \\
C & 2.94838400 & 3.74840400 & -2.31380700 \\
C & 2.86077900 & 2.87337200 & -3.55910800 \\
H & 2.12797900 & 4.46947300 & -2.18975200 \\
H & 3.92845000 & 4.21827200 & -2.14179600 \\
H & 2.61676200 & 1.81104700 & -3.23950000 \\
C & 4.51664500 & 0.24812000 & 4.64691100 \\
& 5.04619500 & 0.47679900 & 3.37139200 \\
C & 4.39729000 & 1.36087800 & 2.51171500 \\
\hline & 3.22428200 & 2.01863700 & 2.91990100
\end{tabular}




$\begin{array}{lrrr}\text { C } & 2.70521000 & 1.79753700 & 4.20799400 \\ \text { C } & 3.35235400 & 0.90784700 & 5.06375900 \\ \text { H } & 2.94432500 & 0.72076600 & 6.05983100 \\ \text { H } & 5.01517800 & -0.45017900 & 5.32431400 \\ \text { H } & 5.95270200 & -0.02755200 & 3.02378000 \\ \text { H } & 4.80553000 & 1.55868100 & 1.52161100 \\ \text { H } & 1.79080800 & 2.30550100 & 4.51721600 \\ \text { C } & 2.53950000 & 2.90700400 & 1.96805400 \\ \text { O } & 1.66567500 & 3.73567500 & 2.48747600 \\ \text { C } & 0.89353500 & 4.58946200 & 1.59753100 \\ \text { C } & -0.19762200 & 5.23384600 & 2.42112500 \\ \text { H } & 1.58428600 & 5.32807000 & 1.16013500 \\ \text { H } & 0.49607000 & 3.97343000 & 0.77789800 \\ \text { H } & 0.22477900 & 5.82632400 & 3.24672300 \\ \text { H } & -0.86950700 & 4.47143000 & 2.84386300 \\ \text { H } & -0.79417600 & 5.90191500 & 1.78141300 \\ \text { O } & 2.76157700 & 2.89715700 & 0.74396500 \\ \text { C } & 4.19341900 & 2.75824000 & -4.31190300 \\ \text { H } & 4.12343900 & 2.02521400 & -5.13102300 \\ \text { H } & 4.46083300 & 3.73501400 & -4.74639700 \\ \text { H } & 5.00472700 & 2.44697300 & -3.63846400 \\ \text { C } & 1.69984100 & 3.25601300 & -4.48791800 \\ \text { H } & 1.88328700 & 4.25501500 & -4.91555300 \\ \text { H } & 1.60322900 & 2.53888100 & -5.31861700 \\ \text { H } & 0.74600300 & 3.28021800 & -3.94238600\end{array}$

$\begin{array}{lrrr}\text { MgCl }_{2} \text {-Ti-isobutyl-DiBS-Mg2-M } & \\ \text { Cl } & -5.15968100 & -3.73801300 & 1.49970200 \\ \text { Mg } & -3.73774900 & -5.14635800 & -0.00780000 \\ \text { Cl } & -6.71103100 & -2.05411000 & -1.07106000 \\ \text { Mg } & -4.63108500 & -1.62674400 & 0.25889800 \\ \text { Cl } & -1.65781400 & -4.71900900 & 1.32217000 \\ \text { Cl } & 0.95067700 & -2.18038300 & 1.41136400 \\ \text { Cl } & -2.55114400 & -1.19938300 & 1.58892700 \\ \text { Cl } & 0.29269200 & -4.01608100 & -1.42611700 \\ \text { Mg } & 2.37260600 & -3.58873200 & -0.09615600 \\ \text { Cl } & -3.20916900 & -3.03509100 & -1.24858300 \\ \text { Mg } & -1.12928600 & -2.60771700 & 0.08137200 \\ \text { Cl } & -0.60064000 & -0.49647100 & -1.15940900 \\ \text { Mg } & 1.47963000 & -0.06916500 & 0.17071200 \\ \text { Cl } & 4.45246900 & -3.16135600 & 1.23382800 \\ \text { Cl } & 7.06104600 & -0.62271900 & 1.32309300 \\ \text { Cl } & 3.53510600 & 0.45062300 & 1.59148800 \\ \text { Cl } & 6.40316900 & -2.45842300 & -1.51439700\end{array}$




\begin{tabular}{|c|c|c|c|}
\hline Mg & 8.48300000 & -2.03111000 & -0.18448900 \\
\hline $\mathrm{Cl}$ & 2.90121900 & -1.47742000 & -1.33701700 \\
\hline Mg & 4.98106000 & -1.05014700 & -0.00715900 \\
\hline $\mathrm{Cl}$ & 5.43743300 & 1.14563300 & -1.31878000 \\
\hline Mg & 7.58940500 & 1.48841300 & 0.08220400 \\
\hline $\mathrm{Cl}$ & 9.66959400 & 1.91585500 & 1.41217200 \\
\hline $\mathrm{Cl}$ & 9.01158400 & 0.08018600 & -1.42529600 \\
\hline $\mathrm{Ti}$ & 4.01316300 & 2.61320300 & 0.28548200 \\
\hline $\mathrm{Cl}$ & 6.13953400 & 2.96886500 & 1.40917300 \\
\hline $\mathrm{Cl}$ & 1.81708800 & 2.14328200 & -0.73230200 \\
\hline $\mathrm{C}$ & 3.53280200 & 4.61863600 & 0.45146100 \\
\hline C & 4.19437500 & 4.80644700 & -0.90011300 \\
\hline $\mathrm{H}$ & 2.44863600 & 4.79627200 & 0.47140400 \\
\hline $\mathrm{H}$ & 4.04983800 & 5.10001600 & 1.29327900 \\
\hline $\mathrm{H}$ & 4.59175500 & 3.78522200 & -1.25562400 \\
\hline C & 3.22401300 & 5.22422600 & -2.01370200 \\
\hline $\mathrm{H}$ & 2.86263300 & 6.24603600 & -1.81738000 \\
\hline $\mathrm{H}$ & 3.72041700 & 5.21836100 & -2.99686200 \\
\hline $\mathrm{H}$ & 2.35477100 & 4.55323600 & -2.06098500 \\
\hline C & 5.45094000 & 5.68718900 & -0.85879300 \\
\hline $\mathrm{H}$ & 6.14793200 & 5.34754300 & -0.07996500 \\
\hline $\mathrm{H}$ & 5.97496100 & 5.67865000 & -1.82779200 \\
\hline $\mathrm{H}$ & 5.16094900 & 6.72613700 & -0.63596200 \\
\hline C & -7.05926000 & 2.66952700 & -0.44676300 \\
\hline $\mathrm{H}$ & -7.32147300 & 2.92700900 & -1.48162300 \\
\hline C & -5.56418900 & 2.20328000 & -0.49503800 \\
\hline $\mathrm{H}$ & -4.95709000 & 3.12156300 & -0.49467000 \\
\hline C & -7.96323300 & 1.52365700 & -0.00755600 \\
\hline C & -5.20481600 & 1.44963600 & 0.76989300 \\
\hline 0 & -4.97608200 & 2.21653600 & 1.81157100 \\
\hline 0 & -8.68805400 & 1.03393500 & -1.00969100 \\
\hline C & -4.72231400 & 1.58252200 & 3.10041000 \\
\hline $\mathrm{H}$ & -4.05440100 & 0.72462600 & 2.93956800 \\
\hline $\mathrm{H}$ & -4.18613900 & 2.35512500 & 3.66833700 \\
\hline C & -6.02291400 & 1.18070100 & 3.76990100 \\
\hline $\mathrm{H}$ & -6.66856300 & 2.05651200 & 3.93427600 \\
\hline $\mathrm{H}$ & -6.57884800 & 0.46480000 & 3.14990600 \\
\hline $\mathrm{H}$ & -5.80051800 & 0.71775200 & 4.74433200 \\
\hline C & -9.53554400 & -0.10577700 & -0.72571600 \\
\hline $\mathrm{H}$ & -8.96043900 & -0.82259900 & -0.12400700 \\
\hline $\mathrm{H}$ & -10.39602600 & 0.24991900 & -0.13385000 \\
\hline C & -9.94497300 & -0.70516500 & -2.05200100 \\
\hline $\mathrm{H}$ & -10.62137100 & -1.55742100 & -1.88126600 \\
\hline $\mathrm{H}$ & -10.46424700 & 0.03298100 & -2.68366700 \\
\hline
\end{tabular}




$\begin{array}{llll}\text { H } & -9.05164300 & -1.07356900 & -2.57696200 \\ \text { O } & -8.01276000 & 1.12516300 & 1.13605800 \\ \text { O } & -5.13750600 & 0.22610200 & 0.83441200 \\ \text { C } & -7.27838100 & 3.89640100 & 0.45501000 \\ \text { H } & -6.66836600 & 4.72453600 & 0.05002800 \\ \text { H } & -6.88354300 & 3.67596800 & 1.45706100 \\ \text { C } & -8.73880100 & 4.36492100 & 0.58834200 \\ \text { H } & -9.29561800 & 3.56354300 & 1.10712500 \\ \text { C } & -8.80466200 & 5.61767900 & 1.47000700 \\ \text { H } & -9.84669700 & 5.93099800 & 1.64370500 \\ \text { H } & -8.33608200 & 5.44321000 & 2.45229600 \\ \text { H } & -8.27703900 & 6.46216900 & 0.99322100 \\ \text { C } & -9.41218800 & 4.60822800 & -0.76869600 \\ \text { H } & -9.50073000 & 3.68166200 & -1.35660900 \\ \text { H } & -10.42931200 & 5.01097800 & -0.63586400 \\ \text { H } & -8.84038700 & 5.33950900 & -1.36685200 \\ \text { C } & -5.27607100 & 1.36138700 & -1.74697300 \\ \text { H } & -5.89708100 & 0.45148800 & -1.70707900 \\ \text { H } & -5.62488600 & 1.93575800 & -2.62248200 \\ \text { C } & -3.80535800 & 0.96980500 & -1.98346000 \\ \text { H } & -3.42326100 & 0.43026900 & -1.09628900 \\ \text { C } & -2.88900800 & 2.18415000 & -2.17472300 \\ \text { H } & -1.85249900 & 1.86183800 & -2.35344600 \\ \text { H } & -3.21751600 & 2.78861100 & -3.03855500 \\ \text { H } & -2.87430400 & 2.84118300 & -1.28970200 \\ \text { C } & -3.71038000 & 0.01139000 & -3.17653900 \\ \text { H } & -4.02156400 & 0.51855300 & -4.10620600 \\ \text { H } & -2.67802500 & -0.34646100 & -3.30725300 \\ \text { H } & -4.36607000 & -0.86243500 & -3.03591800 \\ & & & \end{array}$

\section{$\mathrm{MgCl}_{2}$-Ti-isobutyl-DiBS-Mg2-C}

$\begin{array}{lrrr}\text { Cl } & -5.79211900 & -3.18292800 & 1.30042000 \\ \text { Mg } & -4.49666500 & -4.62579800 & -0.28653900 \\ \text { Cl } & -7.17988100 & -1.22666100 & -1.16864000 \\ \text { Mg } & -5.07506700 & -1.06246600 & 0.17818100 \\ \text { Cl } & -2.39045100 & -4.45938600 & 1.06047400 \\ \text { Cl } & 0.43264400 & -2.17099700 & 1.28478100 \\ \text { Cl } & -2.97000600 & -0.89449400 & 1.52399200 \\ \text { Cl } & -0.37809100 & -3.78033900 & -1.64894600 \\ \text { Mg } & 1.72799300 & -3.61393100 & -0.30202400 \\ \text { Cl } & -3.77956400 & -2.50389400 & -1.40903600 \\ \text { Mg } & -1.67346600 & -2.33756400 & -0.06198300 \\ \text { Cl } & -0.95648300 & -0.21569500 & -1.18462900 \\ \text { Mg } & 1.15005200 & -0.04936100 & 0.16252700\end{array}$




\begin{tabular}{|c|c|c|c|}
\hline $\mathrm{Cl}$ & 3.83402400 & -3.44749300 & 1.04491900 \\
\hline $\mathrm{Cl}$ & 6.65727200 & -1.15922500 & 1.26946100 \\
\hline $\mathrm{Cl}$ & 3.24383900 & 0.21456300 & 1.60230200 \\
\hline $\mathrm{Cl}$ & 5.84668800 & -2.76845400 & -1.66435700 \\
\hline Mg & 7.95268400 & -2.60207200 & -0.31749100 \\
\hline $\mathrm{Cl}$ & 2.44511000 & -1.49203100 & -1.42456800 \\
\hline Mg & 4.55111000 & -1.32569100 & -0.07775200 \\
\hline $\mathrm{Cl}$ & 5.20519700 & 0.88779800 & -1.27117600 \\
\hline Mg & 7.37412100 & 0.96251600 & 0.14688500 \\
\hline $\mathrm{Cl}$ & 9.48048600 & 1.12899100 & 1.49381900 \\
\hline $\mathrm{Cl}$ & 8.66977300 & -0.48019300 & -1.43997600 \\
\hline $\mathrm{Ti}$ & 3.91225500 & 2.38809800 & 0.40993500 \\
\hline $\mathrm{Cl}$ & 6.06067000 & 2.50719500 & 1.54070300 \\
\hline $\mathrm{Cl}$ & 1.68046200 & 2.16845600 & -0.62676100 \\
\hline C & 3.60088100 & 4.41667700 & 0.67560900 \\
\hline C & 4.27456600 & 4.61752200 & -0.66824400 \\
\hline $\mathrm{H}$ & 2.53422100 & 4.67831400 & 0.70815400 \\
\hline $\mathrm{H}$ & 4.15381700 & 4.81440200 & 1.53803300 \\
\hline $\mathrm{H}$ & 4.58089000 & 3.58645600 & -1.07998500 \\
\hline C & 3.34284200 & 5.17664700 & -1.75260000 \\
\hline $\mathrm{H}$ & 3.07252600 & 6.21434700 & -1.50084300 \\
\hline $\mathrm{H}$ & 3.83564300 & 5.17933800 & -2.73760500 \\
\hline $\mathrm{H}$ & 2.41839700 & 4.58739000 & -1.82926000 \\
\hline C & 5.60179900 & 5.38441500 & -0.58844300 \\
\hline $\mathrm{H}$ & 6.26918300 & 4.94495700 & 0.16575200 \\
\hline $\mathrm{H}$ & 6.12103300 & 5.38365600 & -1.56006700 \\
\hline $\mathrm{H}$ & 5.40218700 & 6.43073100 & -0.30811800 \\
\hline C & -5.63247700 & 2.63470300 & -0.51213900 \\
\hline $\mathrm{H}$ & -5.37266400 & 3.65885700 & -0.81524700 \\
\hline C & -5.45126800 & 2.57841000 & 1.03414200 \\
\hline $\mathrm{H}$ & -5.93546500 & 3.48630000 & 1.41940100 \\
\hline C & -4.68116800 & 1.72215000 & -1.26282100 \\
\hline C & -6.21769500 & 1.42044500 & 1.64660900 \\
\hline 0 & -7.25728900 & 1.80429600 & 2.36491800 \\
\hline 0 & -4.10683000 & 2.33227400 & -2.28399600 \\
\hline C & -8.12162100 & 0.76789400 & 2.91541100 \\
\hline $\mathrm{H}$ & -7.48851200 & -0.03246200 & 3.32534100 \\
\hline $\mathrm{H}$ & -8.64477500 & 1.27638000 & 3.73680400 \\
\hline C & -9.08046600 & 0.23704000 & 1.86467000 \\
\hline $\mathrm{H}$ & -9.67430900 & 1.05737500 & 1.43225100 \\
\hline $\mathrm{H}$ & -8.54962000 & -0.28665800 & 1.05387100 \\
\hline $\mathrm{H}$ & -9.77309600 & -0.47707200 & 2.33817900 \\
\hline C & -3.23419600 & 1.59416900 & -3.18349900 \\
\hline $\mathrm{H}$ & -3.05408500 & 0.59407600 & -2.77141400 \\
\hline
\end{tabular}




$\begin{array}{llll}\text { H } & -3.79069200 & 1.50183600 & -4.12933200 \\ \text { C } & -1.94846900 & 2.37480900 & -3.35136200 \\ \text { H } & -1.41177000 & 2.43954200 & -2.39387800 \\ \text { H } & -1.29347400 & 1.85235100 & -4.06544300 \\ \text { O } & -4.47790700 & 0.54878600 & -0.99404700 \\ \text { O } & -5.93316400 & 0.23954400 & 1.51164000 \\ \text { C } & -7.08579700 & 2.32395900 & -0.94110500 \\ \text { H } & -7.74590300 & 3.04157600 & -0.42199000 \\ \text { H } & -7.34493900 & 1.31400600 & -0.58844600 \\ \text { C } & -7.37312200 & 2.35458800 & -2.45129100 \\ \text { H } & -6.71674900 & 1.60364400 & -2.92732600 \\ \text { C } & -8.81459300 & 1.89333800 & -2.69658100 \\ \text { H } & -9.04988500 & 1.88320300 & -3.77330500 \\ \text { H } & -8.95934700 & 0.87543800 & -2.30283800 \\ \text { H } & -9.53498400 & 2.56968700 & -2.20316000 \\ \text { C } & -7.09385900 & 3.72152700 & -3.08588200 \\ \text { H } & -6.03243600 & 4.00222800 & -3.00659600 \\ \text { H } & -7.35448500 & 3.71756800 & -4.15680100 \\ \text { H } & -7.69666200 & 4.50993000 & -2.60134200 \\ \text { C } & -3.96781100 & 2.56692800 & 1.45362200 \\ \text { H } & -3.45991200 & 1.72272300 & 0.96671500 \\ \text { H } & -3.50953400 & 3.49256600 & 1.06009100 \\ \text { C } & -3.69400300 & 2.46217300 & 2.96323700 \\ \text { H } & -4.10759500 & 1.49581000 & 3.30493900 \\ \text { C } & -4.35356800 & 3.58694800 & 3.77005700 \\ \text { H } & -4.09700800 & 3.50712800 & 4.83872700 \\ \text { H } & -4.01169100 & 4.57667000 & 3.41909900 \\ \text { H } & -5.45216700 & 3.56076300 & 3.69500100 \\ \text { C } & -2.18019800 & 2.40779300 & 3.20271000 \\ \text { H } & -1.69229900 & 3.33416100 & 2.85124200 \\ \text { H } & -1.94979200 & 2.29238400 & 4.27399300 \\ & -1.73771900 & 1.55378800 & 2.66629400 \\ -2.14491600 & 3.39026200 & -3.72912800\end{array}$

$$
\mathrm{MgCl}_{2} \text {-Ti-isobutyl-DiBS-Mg1(2) }
$$

Cl $\quad 6.83238900 \quad-2.68430500$

$-0.93934400$

Mg $\quad 5.56344400 \quad-4.35004500$

0.43633200

Cl $\quad 7.91550700$

$-0.85284200$

1.76687700

Mg $\quad 5.88573600$

$-0.72523500$

0.30355700

Cl $\quad 3.53364300$

$-4.22297800$

$-1.02717000$

Cl $\quad 0.55730200$

$-2.13730100$

$-1.24759100$

Cl $\quad 3.85625500$

$-0.59915400$

$-1.16002100$

Cl 1.31808500

$-3.93031100$

1.59147700

$\mathrm{Mg}$

$-0.71162000$

$-3.80324500$

0.12805000 


\begin{tabular}{|c|c|c|c|}
\hline $\mathrm{Cl}$ & 4.61681500 & -2.39161500 & 1.67914800 \\
\hline Mg & 2.58700800 & -2.26462500 & 0.21546600 \\
\hline $\mathrm{Cl}$ & 1.64052800 & -0.30606000 & 1.45870300 \\
\hline Mg & -0.38963900 & -0.17907400 & -0.00502606 \\
\hline $\mathrm{Cl}$ & -2.74127600 & -3.67614300 & -1.3353950 \\
\hline $\mathrm{Cl}$ & -5.71770700 & -1.59060100 & -1.5560730 \\
\hline $\mathrm{Cl}$ & -2.46438000 & 0.14117800 & -1.4338740 \\
\hline $\mathrm{Cl}$ & -4.95709800 & -3.38349700 & 1.2831120 \\
\hline Mg & -6.98671800 & -3.25645500 & $-0.180257 \theta$ \\
\hline $\mathrm{Cl}$ & -1.65831000 & -1.84474000 & 1.3709700 \\
\hline Mg & -3.68793800 & -1.71775400 & -0.0923500 \\
\hline $\mathrm{Cl}$ & -4.72683000 & 0.20176700 & 1.32558606 \\
\hline Mg & -6.66410500 & 0.36776600 & -0.3131050 \\
\hline $\mathrm{Cl}$ & -8.69411100 & 0.49487800 & -1.7765600 \\
\hline $\mathrm{Cl}$ & -7.93337600 & -1.29796100 & 1.06261106 \\
\hline $\mathrm{Ti}$ & -3.40435900 & 2.01636600 & 0.0918040 \\
\hline $\mathrm{Cl}$ & -5.38517300 & 2.17224400 & -1.34229306 \\
\hline $\mathrm{Cl}$ & -1.49190700 & 1.57029200 & 1.48600606 \\
\hline C & -3.28994300 & 4.07708400 & 0.24 \\
\hline C & -4.22418800 & 3.96403800 & 1.43209800 \\
\hline $\mathrm{H}$ & -2.28098200 & 4.43624900 & 0.47432406 \\
\hline $\mathrm{H}$ & -3.71712500 & 4.58634900 & -0.63469200 \\
\hline $\mathrm{H}$ & -4.51586200 & 2.86237900 & 1.56106206 \\
\hline C & -3.57406700 & 4.32021300 & $2.7754640 e$ \\
\hline $\mathrm{H}$ & -3.33052800 & 5.39432700 & 2.79672300 \\
\hline H & -4.25698600 & 4.10674700 & 3.61284700 \\
\hline $\mathrm{H}$ & -2.64765900 & 3.75175900 & 2.93199900 \\
\hline C & -5.57124500 & 4.67184700 & 1.23077400 \\
\hline $\mathrm{H}$ & -6.04000600 & 4.37002800 & 0.28389200 \\
\hline H & -6.26811500 & 4.44513400 & 2.05361600 \\
\hline $\mathrm{H}$ & -5.41168300 & 5.76163500 & $1.2086450 e$ \\
\hline C & 2.49183000 & 3.07027600 & -0.59988200 \\
\hline $\mathrm{H}$ & 2.37652700 & 3.89773600 & -1.31913800 \\
\hline C & 4.06692900 & 3.00727200 & -0.47318400 \\
\hline $\mathrm{H}$ & 4.29521400 & 4.03557300 & -0.14848300 \\
\hline C & 1.76052900 & 1.93939500 & -1.33509500 \\
\hline C & 4.65302600 & 2.17423700 & 0.67083000 \\
\hline 0 & 4.39183300 & 2.64454500 & 1.86720400 \\
\hline 0 & 2.21613000 & 1.76379100 & -2.55411400 \\
\hline C & 5.02703300 & 2.02100000 & 3.02971000 \\
\hline $\mathrm{H}$ & 6.03452200 & 1.68421800 & 2.74140900 \\
\hline $\mathrm{H}$ & 5.10343100 & 2.84725700 & $3.7492880 e$ \\
\hline C & 4.19560100 & 0.87629000 & 3.56685506 \\
\hline $\mathrm{H}$ & 3.17051700 & 1.19646500 & $3.801511 e$ \\
\hline
\end{tabular}




\begin{tabular}{|c|c|c|c|}
\hline $\mathrm{H}$ & 4.13396200 & 0.05310800 & 2.84516800 \\
\hline $\mathrm{H}$ & 4.67140000 & 0.48881300 & 4.48097900 \\
\hline C & 1.63620400 & 0.72481900 & -3.38419500 \\
\hline $\mathrm{H}$ & 1.54873500 & -0.18820800 & -2.78321600 \\
\hline $\mathrm{H}$ & 0.62375800 & 1.05000800 & -3.67475200 \\
\hline C & 2.55505000 & 0.52575900 & -4.56735700 \\
\hline $\mathrm{H}$ & 2.13123700 & -0.23738700 & -5.23791200 \\
\hline $\mathrm{H}$ & 2.68184600 & 1.45924500 & -5.13682900 \\
\hline $\mathrm{H}$ & 3.54024300 & 0.17775900 & -4.22377200 \\
\hline 0 & 0.76743200 & 1.34433000 & -0.91892400 \\
\hline 0 & 5.41349500 & 1.22280600 & 0.51143700 \\
\hline C & 1.80416900 & 3.47318600 & 0.71211000 \\
\hline $\mathrm{H}$ & 2.52192800 & 4.06600200 & 1.29767400 \\
\hline $\mathrm{H}$ & 1.60043100 & 2.56878600 & 1.30490500 \\
\hline C & 0.51712400 & 4.30771500 & 0.58527100 \\
\hline $\mathrm{H}$ & -0.26064700 & 3.67100900 & 0.13318000 \\
\hline C & 0.06059700 & 4.70120600 & 1.99527200 \\
\hline $\mathrm{H}$ & -0.88079800 & 5.27031300 & 1.97526500 \\
\hline $\mathrm{H}$ & -0.09417900 & 3.81271600 & 2.62456800 \\
\hline $\mathrm{H}$ & 0.81959200 & 5.33823200 & 2.48134500 \\
\hline C & 0.68302700 & 5.56016900 & -0.28546900 \\
\hline $\mathrm{H}$ & 0.89354400 & 5.32430400 & -1.34104900 \\
\hline $\mathrm{H}$ & -0.23765200 & 6.16556200 & -0.27406100 \\
\hline $\mathrm{H}$ & 1.50362900 & 6.19823700 & 0.08735800 \\
\hline C & 4.82108800 & 2.74931100 & -1.78685100 \\
\hline $\mathrm{H}$ & 4.74463400 & 1.68391400 & -2.03277300 \\
\hline $\mathrm{H}$ & 4.29644400 & 3.28275000 & -2.59552400 \\
\hline C & 6.30081500 & 3.18229500 & -1.79097300 \\
\hline $\mathrm{H}$ & 6.79005300 & 2.76985000 & -0.89159100 \\
\hline C & 6.46138200 & 4.70770500 & -1.76574900 \\
\hline $\mathrm{H}$ & 7.52485100 & 4.99439900 & -1.74475400 \\
\hline $\mathrm{H}$ & 6.00976900 & 5.16356800 & -2.66408700 \\
\hline $\mathrm{H}$ & 5.98525700 & 5.16716500 & -0.88384400 \\
\hline C & 7.00826400 & 2.57792900 & -3.00991200 \\
\hline $\mathrm{H}$ & 6.53775100 & 2.92137500 & -3.94764600 \\
\hline $\mathrm{H}$ & 8.07082700 & 2.86681300 & -3.04207700 \\
\hline $\mathrm{H}$ & 6.95745600 & 1.47704400 & -2.99465000 \\
\hline
\end{tabular}

$\mathrm{MgCl}_{2}$-Ti-i sobutyl-CMDMS-Mg1

$\begin{array}{lrrr}\text { Cl } & 8.17589900 & 0.07697400 & -0.87747800 \\ \text { Mg } & 7.43819700 & -2.26135000 & -0.36181600 \\ \text { Cl } & 8.85958800 & 0.88396400 & 2.39855200 \\ \text { Mg } & 6.80743100 & 1.06697800 & 0.97281800 \\ \text { Cl } & 5.38604200 & -2.07833500 & -1.78755100\end{array}$




\begin{tabular}{|c|c|c|c|}
\hline $\mathrm{Cl}$ & 1.96540700 & -0.90520700 & -1.36305400 \\
\hline $\mathrm{Cl}$ & 4.75527700 & 1.24999300 & -0.45291300 \\
\hline $\mathrm{Cl}$ & 3.27987300 & -3.42665300 & 0.57840800 \\
\hline Mg & 1.22776200 & -3.24364500 & -0.84729100 \\
\hline $\mathrm{Cl}$ & 6.06973000 & -1.27134700 & 1.48848100 \\
\hline Mg & 4.01757300 & -1.08829400 & 0.06272800 \\
\hline $\mathrm{Cl}$ & 2.64909200 & -0.09818700 & 1.91305200 \\
\hline Mg & 0.59674400 & 0.08418400 & 0.48712800 \\
\hline $\mathrm{Cl}$ & -0.82440900 & -3.06057000 & -2.27306800 \\
\hline $\mathrm{Cl}$ & -4.24505700 & -1.88755400 & -1.84852700 \\
\hline $\mathrm{Cl}$ & -1.68446100 & 0.31127700 & -0.77818400 \\
\hline $\mathrm{Cl}$ & -2.93072400 & -4.40892600 & 0.09280900 \\
\hline Mg & -4.98276900 & -4.22592700 & -1.33284100 \\
\hline $\mathrm{Cl}$ & -0.14087200 & -2.25363600 & 1.00303900 \\
\hline Mg & -2.19261100 & -2.07051800 & -0.42248100 \\
\hline $\mathrm{Cl}$ & -3.61427700 & -1.22457300 & 1.58768800 \\
\hline Mg & -5.61342400 & -0.89758700 & 0.00174700 \\
\hline $\mathrm{Cl}$ & -7.66569500 & -0.71460100 & -1.42392500 \\
\hline $\mathrm{Cl}$ & -6.35122100 & -3.23592000 & 0.51749100 \\
\hline $\mathrm{Ti}$ & -2.89226100 & 1.24343200 & 1.36730600 \\
\hline $\mathrm{Cl}$ & -4.97986700 & 1.47109300 & 0.09463200 \\
\hline $\mathrm{Cl}$ & -0.73616000 & 0.92548200 & 2.43340300 \\
\hline C & -3.33974800 & 2.87806200 & 2.56783800 \\
\hline C & -3.92902400 & 1.88995900 & 3.55190800 \\
\hline $\mathrm{H}$ & -2.44563100 & 3.41080100 & 2.92038000 \\
\hline $\mathrm{H}$ & -4.06654600 & 3.55728600 & 2.10605500 \\
\hline $\mathrm{H}$ & -3.77050800 & 0.83142200 & 3.14564200 \\
\hline C & -3.20636900 & 1.85797900 & 4.90567700 \\
\hline $\mathrm{H}$ & -3.37999000 & 2.80776100 & 5.43637800 \\
\hline $\mathrm{H}$ & -3.57938100 & 1.03672200 & 5.53793600 \\
\hline $\mathrm{H}$ & -2.12326400 & 1.72687900 & 4.77185100 \\
\hline C & -5.45312600 & 2.00051500 & 3.69866900 \\
\hline $\mathrm{H}$ & -5.94700400 & 1.98076400 & 2.71637600 \\
\hline $\mathrm{H}$ & -5.85571600 & 1.17668800 & 4.30937500 \\
\hline $\mathrm{H}$ & -5.70857700 & 2.95092400 & 4.19376800 \\
\hline C & -0.83190900 & 4.09661500 & -1.39988000 \\
\hline C & -1.32949800 & 4.73617900 & -2.73010900 \\
\hline C & -2.03862300 & 3.64014000 & -0.55824000 \\
\hline $\mathrm{H}$ & -0.31505400 & 4.88467300 & -0.82130400 \\
\hline C & -2.69795900 & 5.43675600 & -2.57335700 \\
\hline $\mathrm{H}$ & -1.41105700 & 3.97126700 & -3.52148200 \\
\hline $\mathrm{H}$ & -0.56906700 & 5.45518500 & -3.07549100 \\
\hline C & -2.91229200 & 4.84048400 & -0.12769500 \\
\hline $\mathrm{H}$ & -2.64296800 & 2.93161400 & -1.14428900 \\
\hline
\end{tabular}




$\begin{array}{lrrr}\text { H } & -1.67536900 & 3.09887800 & 0.34249000 \\ \text { C } & -2.87053000 & 5.98923200 & -1.15679700 \\ \text { H } & -3.51266200 & 4.71945100 & -2.77486100 \\ \text { H } & -2.79965600 & 6.23499000 & -3.32601400 \\ \text { H } & -2.58305700 & 5.20936400 & 0.85708900 \\ \text { H } & -3.94889100 & 4.49023400 & -0.00081200 \\ \text { H } & -2.03020200 & 6.66952200 & -0.93271800 \\ \text { H } & -3.78579100 & 6.59735000 & -1.07887600 \\ \text { C } & 0.27758600 & 1.65947600 & -3.14486500 \\ \text { H } & 1.02006000 & 0.85049900 & -3.12999800 \\ \text { H } & -0.72258100 & 1.20836500 & -3.12426300 \\ \text { H } & 0.38886800 & 2.21192500 & -4.09214000 \\ \text { O } & 1.83033200 & 3.86671000 & -2.13258100 \\ \text { O } & 0.95163600 & 2.05070700 & -0.27563800 \\ \text { C } & 3.01517900 & 3.53860400 & -2.83591100 \\ \text { H } & 3.51831300 & 4.47509000 & -3.12413400 \\ \text { H } & 3.70334600 & 2.94515600 & -2.21384600 \\ \text { H } & 2.80210400 & 2.96760800 & -3.75828000 \\ \text { C } & 1.50309000 & 2.92306000 & 0.75047300 \\ \text { H } & 1.93486400 & 3.81976100 & 0.28655700 \\ \text { H } & 0.70847500 & 3.19457000 & 1.46008300 \\ \text { H } & 2.29236600 & 2.37369600 & 1.27546000 \\ \text { Si } & 0.55087800 & 2.87304200 & -1.75790200\end{array}$

\section{$\mathrm{MgCl}_{2}$-Ti-i sobutyl-CMDMS-Mg2}

$\begin{array}{lrrr}\text { Cl } & -6.91902100 & -2.26392500 & 1.37318600 \\ \text { Mg } & -5.77261600 & -3.94509800 & -0.08881600 \\ \text { Cl } & -8.08602800 & -0.36236600 & -1.24850600 \\ \text { Mg } & -5.98055500 & -0.31544200 & 0.10884000 \\ \text { Cl } & -3.66704600 & -3.89816500 & 1.26835800 \\ \text { Cl } & -0.62294600 & -1.90269000 & 1.36111500 \\ \text { Cl } & -3.87489900 & -0.26846200 & 1.46584700 \\ \text { Cl } & -1.58215500 & -3.63077600 & -1.45806800 \\ \text { Mg } & 0.52345000 & -3.58384300 & -0.10086400 \\ \text { Cl } & -4.83411800 & -1.99652000 & -1.35330900 \\ \text { Mg } & -2.72855400 & -1.94963500 & 0.00393300 \\ \text { Cl } & -1.79004600 & -0.00110400 & -1.26051800 \\ \text { Mg } & 0.31530700 & 0.04560400 & 0.09664900 \\ \text { Cl } & 2.62893000 & -3.53688200 & 1.25628500 \\ \text { Cl } & 5.67306900 & -1.54143800 & 1.34905500 \\ \text { Cl } & 2.40986800 & 0.20163700 & 1.54555900 \\ \text { Cl } & 4.71396300 & -3.26952800 & -1.47006400 \\ \text { Mg } & 6.81941300 & -3.22259100 & -0.11298600 \\ \text { Cl } & 1.46191100 & -1.63533300 & -1.36540100\end{array}$




\begin{tabular}{|c|c|c|c|}
\hline Mg & 3.56734400 & -1.58792800 & -0.00829100 \\
\hline $\mathrm{Cl}$ & 4.45172500 & 0.45839300 & -1.34399000 \\
\hline Mg & 6.61191700 & 0.40693500 & 0.08471000 \\
\hline $\mathrm{Cl}$ & 8.71715700 & 0.45402400 & 1.44171600 \\
\hline $\mathrm{Cl}$ & 7.75789500 & -1.27404300 & $-1.3774570 e$ \\
\hline $\mathrm{Ti}$ & 3.30535900 & 2.20047100 & 0.21104000 \\
\hline $\mathrm{Cl}$ & 5.45254700 & 2.17519700 & 1.35249000 \\
\hline $\mathrm{Cl}$ & 1.09503800 & 2.11181200 & -0.87565900 \\
\hline C & -3.88033800 & 3.22211300 & 0.41695300 \\
\hline C & -2.97757300 & 3.60445000 & -0.79006500 \\
\hline C & -3.89075500 & 4.35656500 & 1.45807500 \\
\hline $\mathrm{H}$ & -3.44222000 & 2.32772700 & 0.89314300 \\
\hline C & -1.76126900 & 4.45521500 & -0.35123300 \\
\hline $\mathrm{H}$ & -3.54890200 & 4.16636100 & -1.55203300 \\
\hline $\mathrm{H}$ & -2.63226700 & 2.67780300 & -1.27263200 \\
\hline C & -2.48057200 & 4.53041600 & 2.07674200 \\
\hline $\mathrm{H}$ & -4.20671100 & 5.29412000 & 0.96696000 \\
\hline $\mathrm{H}$ & -4.62457200 & 4.17323600 & 2.26015400 \\
\hline C & -1.36061700 & 4.13226500 & 1.09060500 \\
\hline $\mathrm{H}$ & -2.00421500 & 5.53068000 & -0.41674200 \\
\hline $\mathrm{H}$ & -0.91787200 & 4.28132900 & -1.03718700 \\
\hline $\mathrm{H}$ & -2.39888000 & 3.91873700 & 2.99071300 \\
\hline $\mathrm{H}$ & -2.34683600 & 5.57900600 & 2.39264300 \\
\hline $\mathrm{H}$ & -1.15905500 & 3.04883300 & 1.16347000 \\
\hline $\mathrm{H}$ & -0.41593500 & 4.63352400 & 1.35954400 \\
\hline C & -6.72914000 & 3.72064200 & -0.97061600 \\
\hline $\mathrm{H}$ & -7.58488700 & 3.12934300 & -1.33441400 \\
\hline $\mathrm{H}$ & -7.09457200 & 4.46268800 & -0.24219700 \\
\hline $\mathrm{H}$ & -6.28138400 & 4.26824100 & -1.81495100 \\
\hline 0 & -5.20350800 & 1.22041900 & -1.16221200 \\
\hline 0 & -6.30318000 & 1.61012600 & 1.00868600 \\
\hline C & -5.18959800 & 1.14319800 & -2.59054300 \\
\hline $\mathrm{H}$ & -4.32263000 & 0.53803800 & -2.88906700 \\
\hline $\mathrm{H}$ & -6.12555700 & 0.66661400 & -2.91886800 \\
\hline $\mathrm{H}$ & -5.09584900 & 2.14614300 & -3.03645700 \\
\hline C & -6.42956500 & 1.84621400 & 2.40655800 \\
\hline $\mathrm{H}$ & -5.45766700 & 1.71807800 & 2.90947200 \\
\hline $\mathrm{H}$ & -6.82676800 & 2.85567200 & 2.60343800 \\
\hline $\mathrm{H}$ & -7.13612400 & 1.10593700 & 2.80837400 \\
\hline Si & -5.52027500 & 2.55439600 & -0.17574100 \\
\hline C & 3.18896700 & 4.26070700 & 0.34709200 \\
\hline $\mathrm{H}$ & 2.15197600 & 4.62520700 & 0.35398400 \\
\hline $\mathrm{H}$ & 3.77545500 & 4.65891100 & 1.18689500 \\
\hline C & 3.88066600 & 4.31110200 & -1.00198400 \\
\hline
\end{tabular}




$\begin{array}{llll}\text { C } & 5.27808400 & 4.94472000 & -0.95976400 \\ \text { C } & 3.01017700 & 4.89513200 & -2.12359000 \\ \text { H } & 4.08165500 & 3.23121500 & -1.34920900 \\ \text { H } & 5.79352300 & 4.83641100 & -1.92736300 \\ \text { H } & 5.89924900 & 4.48437200 & -0.17897600 \\ \text { H } & 5.18457300 & 6.02001500 & -0.74042500 \\ \text { H } & 3.50062600 & 4.78922500 & -3.10402800 \\ \text { H } & 2.84575400 & 5.96794500 & -1.93562500 \\ \text { H } & 2.03093200 & 4.39878700 & -2.17126700\end{array}$

$\mathrm{MgCl}_{2}$-Ti-isobutyl-CMDMS-Ti

$\begin{array}{lrrr}\text { Cl } & 8.61123200 & 1.76944400 & -1.42953500 \\ \text { Mg } & 8.41620200 & -0.66581900 & -0.87336900 \\ \text { Cl } & 9.33680500 & 2.73579300 & 1.79393400 \\ \text { Mg } & 7.19784000 & 2.47127600 & 0.51627200 \\ \text { Cl } & 6.27724000 & -0.93033700 & -2.15103200 \\ \text { Cl } & 2.72485500 & -0.49303200 & -1.48290100 \\ \text { Cl } & 5.05887600 & 2.20676100 & -0.76139000 \\ \text { Cl } & 4.66881800 & -2.66376600 & 0.35094100 \\ \text { Mg } & 2.52991600 & -2.92827900 & -0.92667900 \\ \text { Cl } & 7.00281100 & 0.03601300 & 1.07243800 \\ \text { Mg } & 4.86385300 & -0.22841600 & -0.20525400 \\ \text { Cl } & 3.45046400 & 0.47335000 & 1.74056500 \\ \text { Mg } & 1.31086500 & 0.20846000 & 0.46288100 \\ \text { Cl } & 0.39095000 & -3.19273600 & -2.20436400 \\ \text { Cl } & -3.16150100 & -2.75543800 & -1.53628100 \\ \text { Cl } & -0.80276700 & -0.05083200 & -0.88691100 \\ \text { Cl } & -1.21764500 & -4.92622800 & 0.29755300 \\ \text { Mg } & -3.35650500 & -5.19074600 & -0.98008400 \\ \text { Cl } & 1.11647100 & -2.22644700 & 1.01920400 \\ \text { Mg } & -1.02231400 & -2.49108200 & -0.25868600 \\ \text { Cl } & -2.35792900 & -1.71050000 & 1.71092300 \\ \text { Mg } & -4.57432400 & -2.05337800 & 0.40962100 \\ \text { Cl } & -6.71382700 & -2.31818600 & -0.86808100 \\ \text { Cl } & -4.76992000 & -4.48890300 & 0.96576300 \\ \text { Ti } & -2.47811800 & 0.96460700 & 1.40660400 \\ \text { Cl } & -4.81917700 & 0.31182900 & 0.93771600 \\ \text { Cl } & -0.08709700 & 1.36237400 & 2.06400300 \\ \text { C } & -3.10170300 & 2.31046600 & 2.85486100 \\ \text { C } & -3.11863200 & 1.19436800 & 3.88964100 \\ \text { H } & -2.35070800 & 3.09009100 & 3.04565300 \\ \text { H } & -4.08413400 & 2.75508500 & 2.63972500 \\ \text { C } & -2.79443000 & 0.22886200 & 3.38893600 \\ & -2.09893800 & 1.39688700 & 5.02024800\end{array}$




\begin{tabular}{|c|c|c|c|}
\hline $\mathrm{H}$ & -2.38674000 & 2.27249300 & 5.62452400 \\
\hline $\mathrm{H}$ & -2.06500800 & 0.51849100 & 5.68432300 \\
\hline $\mathrm{H}$ & -1.08806100 & 1.56686700 & 4.62438000 \\
\hline C & -4.52129900 & 0.89225400 & 4.43599300 \\
\hline $\mathrm{H}$ & -5.23850300 & 0.71847500 & 3.62214500 \\
\hline $\mathrm{H}$ & -4.51092800 & 0.00246800 & 5.08567200 \\
\hline $\mathrm{H}$ & -4.87598100 & 1.74757200 & 5.03365200 \\
\hline C & -4.81765100 & 3.78329300 & -1.43728500 \\
\hline C & -5.66853300 & 3.91611400 & -2.73371900 \\
\hline C & -5.72654100 & 3.52404400 & -0.21827900 \\
\hline $\mathrm{H}$ & -4.29775700 & 4.74474400 & -1.26877600 \\
\hline C & -7.07772300 & 4.48296300 & -2.44720000 \\
\hline $\mathrm{H}$ & -5.77621900 & 2.93918900 & -3.23708900 \\
\hline $\mathrm{H}$ & -5.13198900 & 4.56620900 & -3.44382500 \\
\hline C & -6.66102400 & 4.72590800 & 0.04207300 \\
\hline $\mathrm{H}$ & -6.31986100 & 2.61328400 & -0.40152400 \\
\hline $\mathrm{H}$ & -5.13093100 & 3.30162000 & 0.68012500 \\
\hline C & -7.05226400 & 5.45463800 & -1.26359000 \\
\hline $\mathrm{H}$ & -7.77251000 & 3.65705500 & -2.21554100 \\
\hline $\mathrm{H}$ & -7.47515000 & 4.97212800 & -3.35122500 \\
\hline $\mathrm{H}$ & -6.17119400 & 5.43823500 & 0.72784600 \\
\hline $\mathrm{H}$ & -7.56573800 & 4.37063400 & 0.56315600 \\
\hline $\mathrm{H}$ & -6.32357400 & 6.25602500 & -1.48029400 \\
\hline $\mathrm{H}$ & -8.02700100 & 5.95468400 & -1.14523900 \\
\hline C & -3.98433500 & 0.82642300 & -2.19664900 \\
\hline $\mathrm{H}$ & -3.29923100 & 0.03181500 & -1.88057100 \\
\hline $\mathrm{H}$ & -4.99385000 & 0.56851700 & -1.84803800 \\
\hline $\mathrm{H}$ & -3.98957300 & 0.83680500 & -3.29923900 \\
\hline 0 & -2.35549200 & 3.18174300 & -2.69581200 \\
\hline 0 & -2.60257100 & 2.47924300 & -0.08651200 \\
\hline C & -1.20671800 & 2.55950700 & -3.24017500 \\
\hline $\mathrm{H}$ & -0.87904700 & 3.13582300 & -4.11925100 \\
\hline $\mathrm{H}$ & -0.38040200 & 2.53579700 & -2.50803400 \\
\hline $\mathrm{H}$ & -1.40202700 & 1.51881700 & -3.55255200 \\
\hline C & -1.97847300 & 3.74264000 & 0.25603400 \\
\hline $\mathrm{H}$ & -1.69852100 & 4.27690100 & -0.66229600 \\
\hline $\mathrm{H}$ & -2.67973900 & 4.35074400 & 0.84591000 \\
\hline $\mathrm{H}$ & -1.07631100 & 3.54178200 & 0.84612600 \\
\hline Si & -3.45588600 & 2.52090000 & -1.63905506 \\
\hline
\end{tabular}

$\mathrm{MgCl}_{2}-\mathrm{Ti}$ - isobutyl-DCPDMS-Mg1

$\begin{array}{lrrr}\text { Cl } & -8.26076900 & -0.03000300 & 0.93089100 \\ \text { Mg } & -7.54501400 & -2.28521800 & 0.10641500 \\ \text { Cl } & -8.97768000 & 1.19968400 & -2.20358600\end{array}$




\begin{tabular}{|c|c|c|c|}
\hline Mg & -6.90761900 & 1.18514400 & -0.79213300 \\
\hline $\mathrm{Cl}$ & -5.47497400 & -2.29969100 & 1.51790200 \\
\hline $\mathrm{Cl}$ & -2.05181700 & -1.09923000 & 1.20618600 \\
\hline $\mathrm{Cl}$ & -4.83761100 & 1.17060300 & 0.61928100 \\
\hline $\mathrm{Cl}$ & -3.40606100 & -3.33975600 & -1.02959200 \\
\hline Mg & -1.33612200 & -3.35430700 & 0.38176100 \\
\hline $\mathrm{Cl}$ & -6.19186500 & -1.07007300 & -1.61661100 \\
\hline Mg & -4.12169800 & -1.08470700 & -0.20513900 \\
\hline $\mathrm{Cl}$ & -2.76924000 & 0.13044800 & -1.92790500 \\
\hline Mg & -0.69730700 & 0.11664300 & -0.51696700 \\
\hline $\mathrm{Cl}$ & 0.73399100 & -3.36882500 & 1.79327300 \\
\hline $\mathrm{Cl}$ & 4.15726700 & -2.16823200 & 1.48176400 \\
\hline $\mathrm{Cl}$ & 1.63531600 & 0.18180500 & 0.66375700 \\
\hline $\mathrm{Cl}$ & 2.80303200 & -4.40885200 & -0.75414800 \\
\hline Mg & 4.87301400 & -4.42339700 & 0.65725600 \\
\hline $\mathrm{Cl}$ & 0.01686700 & -2.13926400 & -1.34108000 \\
\hline Mg & 2.08694900 & -2.15390400 & 0.07042600 \\
\hline $\mathrm{Cl}$ & 3.51555800 & -1.09793800 & -1.86737500 \\
\hline Mg & 5.51016200 & -0.95302000 & -0.24116700 \\
\hline $\mathrm{Cl}$ & 7.58048000 & -0.96762000 & 1.17013800 \\
\hline $\mathrm{Cl}$ & 6.22614800 & -3.20823400 & -1.06582700 \\
\hline $\mathrm{Ti}$ & 2.80367100 & 1.30801100 & -1.36881900 \\
\hline $\mathrm{Cl}$ & 4.86850400 & 1.40361300 & -0.03242200 \\
\hline $\mathrm{Cl}$ & 0.62599700 & 1.16632600 & -2.41378100 \\
\hline C & 3.24433300 & 3.10351200 & -2.30963300 \\
\hline C & 3.88975800 & 2.27648100 & -3.40111800 \\
\hline $\mathrm{H}$ & 2.35143000 & 3.66054300 & -2.61927500 \\
\hline $\mathrm{H}$ & 3.93772700 & 3.72598900 & -1.73007500 \\
\hline $\mathrm{H}$ & 3.78279400 & 1.16736400 & -3.13279500 \\
\hline C & 3.17808100 & 2.38225100 & -4.75711500 \\
\hline $\mathrm{H}$ & 3.30648800 & 3.39935500 & -5.16055800 \\
\hline $\mathrm{H}$ & 3.59599900 & 1.66775000 & -5.48386900 \\
\hline $\mathrm{H}$ & 2.10176900 & 2.18227400 & -4.65499500 \\
\hline C & 5.40743200 & 2.47752000 & -3.51260700 \\
\hline $\mathrm{H}$ & 5.89473800 & 2.36005500 & -2.53400100 \\
\hline $\mathrm{H}$ & 5.85573500 & 1.75768200 & -4.21591100 \\
\hline $\mathrm{H}$ & 5.61698600 & 3.49402600 & -3.88207300 \\
\hline C & 1.40558300 & 3.81065200 & 0.79419200 \\
\hline C & 0.91570800 & 4.81303600 & -0.28455100 \\
\hline C & 2.35953600 & 4.66565600 & 1.65638400 \\
\hline $\mathrm{H}$ & 2.03540600 & 3.03832400 & 0.30288400 \\
\hline C & 2.06000700 & 5.85279000 & -0.45937700 \\
\hline $\mathrm{H}$ & 0.00888000 & 5.31956300 & 0.07759000 \\
\hline $\mathrm{H}$ & 0.64875700 & 4.30246700 & -1.22068800 \\
\hline
\end{tabular}




$\begin{array}{lrrr}\text { C } & 3.11227600 & 5.51155200 & 0.61900200 \\ \text { H } & 3.03829900 & 4.06715600 & 2.27777300 \\ \text { H } & 1.76945600 & 5.31264800 & 2.32990500 \\ \text { H } & 2.49295100 & 5.82090700 & -1.47000500 \\ \text { H } & 1.66972700 & 6.87255400 & -0.31685900 \\ \text { H } & 3.92046300 & 4.89780900 & 0.18792000 \\ \text { H } & 3.58652500 & 6.40347400 & 1.05663500 \\ \mathrm{C} & 0.51461900 & 1.94947800 & 3.26483600 \\ \mathrm{C} & -0.02034400 & 0.50688500 & 3.52576100 \\ \mathrm{C} & 2.03179200 & 1.89383700 & 3.60759600 \\ \mathrm{H} & 0.06358400 & 2.60665100 & 4.03035300 \\ \mathrm{C} & 1.15166000 & -0.27776500 & 4.16264700 \\ \mathrm{H} & -0.36115500 & 0.01098900 & 2.61558300 \\ \mathrm{H} & -0.89470600 & 0.54437900 & 4.19437100 \\ \mathrm{C} & 2.11907500 & 0.79550100 & 4.67579100 \\ \mathrm{H} & 2.41176900 & 2.86093200 & 3.96757300 \\ \mathrm{H} & 2.63926800 & 1.61001500 & 2.73451600 \\ \mathrm{H} & 0.81699200 & -0.97343700 & 4.94754700 \\ \mathrm{H} & 1.65160800 & -0.88476100 & 3.39157500 \\ \mathrm{H} & 1.76932800 & 1.19281500 & 5.64558700 \\ \mathrm{H} & 3.14544700 & 0.42342700 & 4.82149500 \\ \mathrm{O} & -1.03358000 & 4.10686700 & 2.17592200 \\ \mathrm{O} & -0.86426700 & 2.00034200 & 0.48979900 \\ \mathrm{C} & -1.73728000 & 2.81644400 & -0.35364400 \\ \mathrm{H} & -1.34384400 & 2.81353100 & -1.37829800 \\ \mathrm{H} & -2.73792100 & 2.37614800 & -0.33957900 \\ \mathrm{H} & -1.78065600 & 3.84286400 & 0.02385400 \\ \mathrm{C} & -2.25162900 & 3.89283100 & 2.86394500 \\ \mathrm{H} & -2.69843500 & 4.87116000 & 3.09994300 \\ \mathrm{H} & -2.97277000 & 3.31658900 & 2.25559400 \\ \mathrm{H} \mathrm{i} & -2.10050900 & 3.34761200 & 3.81458600 \\ & 0.02603100 & 2.90253700 & 1.70351400\end{array}$

$\mathrm{MgCl}_{2}$-Ti-i sobutyl-DCPDMS-Mg2

$\begin{array}{lrrr}\text { Cl } & 6.22267400 & -3.00670300 & -1.35237200 \\ \text { Mg } & 4.93345000 & -4.55453300 & 0.13795100 \\ \text { Cl } & 7.56227200 & -1.17592300 & 1.23697100 \\ \text { Mg } & 5.46601700 & -0.96243200 & -0.11862900 \\ \text { Cl } & 2.83721100 & -4.34037900 & -1.21756600 \\ \text { Cl } & -0.01554600 & -2.08122000 & -1.33936700 \\ \text { Cl } & 3.36968500 & -0.74744000 & -1.47390400 \\ \text { Cl } & 0.79161300 & -3.84309800 & 1.50638400 \\ \text { Mg } & -1.30461600 & -3.62894000 & 0.15086800 \\ \text { Cl } & 4.17676600 & -2.50919800 & 1.37190600\end{array}$




\begin{tabular}{|c|c|c|c|}
\hline $\mathrm{Mg}$ & 2.08076600 & -2.29554100 & 0.01601500 \\
\hline $\mathrm{Cl}$ & 1.32414800 & -0.25040500 & 1.24991000 \\
\hline Mg & -0.77243300 & -0.03632400 & -0.10577600 \\
\hline $\mathrm{Cl}$ & -3.40079700 & -3.41475100 & -1.20467100 \\
\hline $\mathrm{Cl}$ & -6.25363400 & -1.15559400 & -1.32654500 \\
\hline $\mathrm{Cl}$ & -2.84877900 & 0.27306000 & -1.55738900 \\
\hline $\mathrm{Cl}$ & -5.44658300 & -2.91748600 & 1.51921800 \\
\hline Mg & -7.54272700 & -2.70335500 & 0.16375500 \\
\hline $\mathrm{Cl}$ & -2.06117800 & -1.58391300 & 1.38467600 \\
\hline Mg & -4.15732800 & -1.36982500 & 0.02926300 \\
\hline $\mathrm{Cl}$ & -4.85226700 & 0.77175200 & 1.33069400 \\
\hline Mg & -7.00992500 & 0.88928000 & -0.09270600 \\
\hline $\mathrm{Cl}$ & -9.10643800 & 1.10350300 & -1.44823300 \\
\hline $\mathrm{Cl}$ & -8.29925800 & -0.65834100 & 1.39751000 \\
\hline $\mathrm{Ti}$ & -3.56244000 & 2.37166100 & -0.26411800 \\
\hline $\mathrm{Cl}$ & -5.70346700 & 2.51541700 & -1.40461000 \\
\hline $\mathrm{Cl}$ & -1.35855800 & 2.11590300 & 0.81187100 \\
\hline $\mathrm{C}$ & -3.26040200 & 4.40982900 & -0.44449600 \\
\hline C & -3.95699000 & 4.55397400 & 0.89507100 \\
\hline $\mathrm{H}$ & -2.19439300 & 4.67678700 & -0.44901800 \\
\hline $\mathrm{H}$ & -3.80127200 & 4.83825200 & -1.29997900 \\
\hline $\mathrm{H}$ & -4.26305400 & 3.50525700 & 1.26104700 \\
\hline C & -3.04615400 & 5.07636600 & 2.01487100 \\
\hline $\mathrm{H}$ & -2.77710600 & 6.12387600 & 1.80608200 \\
\hline $\mathrm{H}$ & -3.55508700 & 5.04019900 & 2.99094200 \\
\hline $\mathrm{H}$ & -2.11995900 & 4.48917900 & 2.08511200 \\
\hline C & -5.28770700 & 5.31577000 & 0.82409100 \\
\hline $\mathrm{H}$ & -5.94110600 & 4.90283800 & 0.04308400 \\
\hline $\mathrm{H}$ & -5.82173200 & 5.27379600 & 1.78674500 \\
\hline $\mathrm{H}$ & -5.09022600 & 6.37347100 & 0.58853400 \\
\hline C & 4.16744500 & 3.10035200 & -0.78401900 \\
\hline C & 2.72907300 & 2.50924200 & -0.89734100 \\
\hline C & 3.96693000 & 4.43373300 & -0.01776400 \\
\hline $\mathrm{H}$ & 4.52003300 & 3.36668300 & -1.79849800 \\
\hline C & 1.75546200 & 3.69187100 & -0.62566400 \\
\hline $\mathrm{H}$ & 2.57125900 & 1.70268400 & -0.17074400 \\
\hline $\mathrm{H}$ & 2.57575100 & 2.05198300 & -1.88475800 \\
\hline C & 2.63425200 & 4.95355000 & -0.56940700 \\
\hline $\mathrm{H}$ & 4.80320600 & 5.13807300 & -0.14908300 \\
\hline $\mathrm{H}$ & 3.86599000 & 4.24945900 & 1.06828600 \\
\hline $\mathrm{H}$ & 0.95888000 & 3.76788500 & -1.38323100 \\
\hline $\mathrm{H}$ & 1.25029600 & 3.54266500 & 0.34184900 \\
\hline $\mathrm{H}$ & 2.79696000 & 5.35527600 & -1.58521700 \\
\hline $\mathrm{H}$ & 2.19761200 & 5.76341000 & 0.0369480 \\
\hline
\end{tabular}




$\begin{array}{lrrr}\text { C } & 6.87932200 & 3.04068600 & 0.68058400 \\ \text { C } & 8.03536100 & 2.27780200 & 1.37635600 \\ \text { C } & 7.56583100 & 3.84898900 & -0.48160900 \\ \text { H } & 6.38817400 & 3.73300600 & 1.38546300 \\ \text { C } & 9.02594400 & 2.00962800 & 0.23997800 \\ \text { H } & 7.73003900 & 1.34689300 & 1.87341500 \\ \text { H } & 8.49944800 & 2.93624600 & 2.13270100 \\ \text { C } & 9.02520200 & 3.32586800 & -0.55250100 \\ \text { H } & 7.53228200 & 4.93208400 & -0.28687400 \\ \text { H } & 7.05459200 & 3.71269900 & -1.45090300 \\ \text { H } & 10.02501300 & 1.72178200 & 0.60164000 \\ \text { H } & 8.65077200 & 1.16953800 & -0.36678300 \\ \text { H } & 9.69882400 & 4.05017600 & -0.06441800 \\ \text { H } & 9.38036200 & 3.20977200 & -1.58900400 \\ \text { O } & 4.90489300 & 0.80920300 & 1.00200300 \\ \text { O } & 6.06645700 & 0.83002600 & -1.15123500 \\ \text { C } & 6.19446300 & 0.92662900 & -2.56601200 \\ \text { H } & 5.20665400 & 1.04532500 & -3.03880000 \\ \text { H } & 6.85262000 & 1.76492600 & -2.84516100 \\ \text { H } & 6.64465700 & -0.00982100 & -2.92488200 \\ \text { C } & 4.80018400 & 0.82375400 & 2.42799700 \\ \text { H } & 3.78731000 & 0.49512500 & 2.70094500 \\ \text { H } & 5.55524700 & 0.13317500 & 2.83313300 \\ \text { H } & 4.97680000 & 1.83521200 & 2.82779500 \\ \text { Si } & 5.50089800 & 2.00038700 & -0.04270100\end{array}$

$\mathrm{MgCl}_{2}$ - $\mathrm{Ti}$-i sobutyl-DCPDMS-Ti

$\begin{array}{lrrr}\text { Cl } & -8.76402800 & -1.72267400 & -1.58652400 \\ \text { Mg } & -8.62682100 & 0.65389000 & -0.80487300 \\ \text { Cl } & -9.47183500 & -3.00223700 & 1.53000600 \\ \text { Mg } & -7.33765000 & -2.57022100 & 0.29057900 \\ \text { Cl } & -6.49263800 & 1.08590700 & -2.04429900 \\ \text { Cl } & -2.93208900 & 0.67034600 & -1.40662600 \\ \text { Cl } & -5.20346700 & -2.13820700 & -0.94884800 \\ \text { Cl } & -4.92905600 & 2.61492100 & 0.61445800 \\ \text { Mg } & -2.79490800 & 3.04692200 & -0.62495000 \\ \text { Cl } & -7.20044500 & -0.19365800 & 1.07223200 \\ \text { Mg } & -5.06626300 & 0.23831000 & -0.16721700 \\ \text { Cl } & -3.63987300 & -0.60916900 & 1.70993500 \\ \text { Mg } & -1.50534700 & -0.17685900 & 0.47028800 \\ \text { Cl } & -0.66072200 & 3.47887700 & -1.86440500 \\ \text { Cl } & 2.89987200 & 3.06333900 & -1.22674400 \\ \text { Cl } & 0.59169800 & 0.24321400 & -0.89519500 \\ \text { Cl } & 0.90300600 & 5.00798400 & 0.79429300\end{array}$




\begin{tabular}{|c|c|c|c|}
\hline Mg & 3.03705400 & 5.43998000 & -0.44505600 \\
\hline $\mathrm{Cl}$ & -1.36847600 & 2.19939700 & 1.25220500 \\
\hline Mg & 0.76555200 & 2.63135900 & 0.01286500 \\
\hline $\mathrm{Cl}$ & 2.15597300 & 1.59036600 & 1.85860000 \\
\hline Mg & 4.32598200 & 2.21578200 & 0.65038700 \\
\hline $\mathrm{Cl}$ & 6.46042200 & 2.64791200 & -0.58905300 \\
\hline $\mathrm{Cl}$ & 4.46348600 & 4.59242000 & 1.43207100 \\
\hline $\mathrm{Ti}$ & 2.27351500 & -0.96137100 & 1.03050700 \\
\hline $\mathrm{Cl}$ & 4.52783900 & -0.21023600 & 0.48948500 \\
\hline $\mathrm{Cl}$ & -0.08561600 & -1.45031500 & 1.92180100 \\
\hline $\mathrm{C}$ & 2.87942200 & -2.03845900 & 2.70261900 \\
\hline C & 2.79001600 & -1.26481600 & 4.03071300 \\
\hline $\mathrm{H}$ & 2.22497000 & -2.92828900 & 2.69710700 \\
\hline $\mathrm{H}$ & 3.91190600 & -2.37070300 & 2.49497000 \\
\hline $\mathrm{H}$ & 1.90139900 & -0.60644000 & 3.99842200 \\
\hline C & 2.58972300 & -2.22379500 & 5.21479000 \\
\hline $\mathrm{H}$ & 3.43343200 & -2.93113400 & 5.28856700 \\
\hline $\mathrm{H}$ & 2.52392800 & -1.67189800 & 6.16749100 \\
\hline $\mathrm{H}$ & 1.66412400 & -2.80942200 & 5.09734900 \\
\hline C & 4.02653800 & -0.38241900 & 4.24567800 \\
\hline $\mathrm{H}$ & 4.18910100 & 0.29948300 & 3.39826400 \\
\hline $\mathrm{H}$ & 3.92842800 & 0.22658400 & 5.15819700 \\
\hline $\mathrm{H}$ & 4.93320500 & -1.00319800 & 4.34579400 \\
\hline C & 5.09287500 & -3.57867400 & -0.07450200 \\
\hline C & 4.62063900 & -4.78270100 & 0.77511100 \\
\hline C & 6.43326800 & -4.05574400 & -0.71948400 \\
\hline $\mathrm{H}$ & 5.31438000 & -2.74418300 & 0.60576500 \\
\hline C & 5.93854500 & -5.34045100 & 1.32615600 \\
\hline $\mathrm{H}$ & 4.13324500 & -5.53599900 & 0.13347000 \\
\hline $\mathrm{H}$ & 3.90175100 & -4.49629400 & 1.55711700 \\
\hline C & 6.88046200 & -5.29408400 & 0.11004700 \\
\hline $\mathrm{H}$ & 7.17731000 & -3.24596000 & -0.69777300 \\
\hline $\mathrm{H}$ & 6.30946700 & -4.33312000 & -1.77935100 \\
\hline $\mathrm{H}$ & 6.31050900 & -4.67607400 & 2.12581300 \\
\hline $\mathrm{H}$ & 5.83979700 & -6.34945200 & 1.75711100 \\
\hline $\mathrm{H}$ & 7.94235000 & -5.24645800 & 0.39615300 \\
\hline $\mathrm{H}$ & 6.75102900 & -6.21084100 & -0.48808300 \\
\hline C & 4.29469700 & -1.77481800 & -2.67697100 \\
\hline C & 3.37903000 & -0.54679600 & -2.86893400 \\
\hline C & 5.73562100 & -1.18005200 & -2.64130800 \\
\hline $\mathrm{H}$ & 4.21992800 & -2.36562600 & -3.60925800 \\
\hline C & 4.17656600 & 0.32939400 & -3.84287400 \\
\hline $\mathrm{H}$ & 3.24674700 & -0.01227000 & -1.91840400 \\
\hline $\mathrm{H}$ & 2.37103800 & -0.80441500 & -3.23120700 \\
\hline
\end{tabular}




$\begin{array}{lrrr}\text { C } & 5.63958200 & 0.19219100 & -3.37090500 \\ \text { H } & 6.45052200 & -1.86656200 & -3.12083100 \\ \text { H } & 6.08692800 & -1.02660200 & -1.61147700 \\ \text { H } & 4.05686300 & -0.06282900 & -4.86838600 \\ \text { H } & 3.82702000 & 1.37379400 & -3.84733400 \\ \text { H } & 6.34725200 & 0.26108500 & -4.21166900 \\ \text { H } & 5.89406200 & 1.00041900 & -2.66901500 \\ \text { O } & 3.34346700 & -4.44390800 & -2.05732800 \\ \text { O } & 2.39958500 & -2.46602000 & -0.44371600 \\ \text { C } & 1.15092400 & -3.07417200 & -0.86790800 \\ \text { H } & 0.36910500 & -2.79432500 & -0.15568100 \\ \text { H } & 0.88089800 & -2.71128900 & -1.87102500 \\ \text { H } & 1.25546600 & -4.16609400 & -0.87269500 \\ \text { C } & 2.73865700 & -4.72979700 & -3.29557600 \\ \text { H } & 3.14785700 & -5.67498700 & -3.68761000 \\ \text { H } & 1.64657000 & -4.85188100 & -3.18099500 \\ \text { H } & 2.91322700 & -3.94161900 & -4.05024000 \\ \text { Si } & 3.84607100 & -3.02369600 & -1.34322800\end{array}$

\section{Propylene Insertion}

$\begin{array}{lrrr}\mathrm{MgCl}_{2} \text {-Ti-isobutyl-Propylene-1,2-re-coordination } \\ \mathrm{Cl} & 8.18056000 & 0.00471600 & -1.46617800 \\ \mathrm{Mg} & 7.48220600 & -1.80466600 & 0.12010800 \\ \mathrm{Cl} & 8.87842400 & 2.29332100 & 1.00896000 \\ \mathrm{Mg} & 6.81233600 & 1.74945400 & -0.29987600 \\ \mathrm{Cl} & 5.41611900 & -2.34853500 & -1.18872800 \\ \mathrm{Cl} & 1.98180700 & -1.14765800 & -1.33127000 \\ \mathrm{Cl} & 4.74625000 & 1.20558700 & -1.60871200 \\ \mathrm{Cl} & 3.34954200 & -2.41317500 & 1.56386100 \\ \mathrm{Mg} & 1.28348300 & -2.95703700 & 0.25504500 \\ \mathrm{Cl} & 6.11398300 & -0.05992700 & 1.28641100 \\ \mathrm{Mg} & 4.04790500 & -0.60377200 & -0.02245000 \\ \mathrm{Cl} & 2.67966500 & 1.14095900 & 1.14388200 \\ \mathrm{Mg} & 0.61335000 & 0.59678800 & -0.16507600 \\ \mathrm{Cl} & -0.78256600 & -3.50086300 & -1.05380800 \\ \mathrm{Cl} & -4.21692900 & -2.30000600 & -1.19642700 \\ \mathrm{Cl} & -1.47389700 & 0.09339900 & -1.50893400 \\ \mathrm{Cl} & -2.84931500 & -3.56556600 & 1.69871600 \\ \mathrm{Mg} & -4.91530500 & -4.10942400 & 0.38993000 \\ \mathrm{Cl} & -0.08483300 & -1.21224400 & 1.42144700 \\ \mathrm{Mg} & -2.15072300 & -1.75601900 & 0.11251300 \\ \mathrm{Cl} & -3.49091200 & 0.07829500 & 1.32850600 \\ \mathrm{Mg} & -5.58496400 & -0.55534900 & -0.02993400 \\ \mathrm{Cl} & -7.65125400 & -1.09916300 & -1.33887700\end{array}$




$\begin{array}{lrrr}\text { Cl } & -6.28352600 & -2.36467600 & 1.55624400 \\ \text { Ti } & -2.90824600 & 2.10349300 & -0.18444600 \\ \text { Cl } & -4.94069900 & 1.46216400 & -1.31530300 \\ \text { Cl } & -0.56071300 & 2.60363600 & 0.49176400 \\ \text { C } & -3.53328000 & 3.50714300 & 1.19333700 \\ \text { H } & -4.51155500 & 3.66860800 & 0.68979800 \\ \text { H } & -3.73904100 & 2.88173500 & 2.08330800 \\ \text { C } & -2.83938300 & 4.82453200 & 1.58116900 \\ \text { C } & -2.11362200 & 4.68360200 & 2.92423700 \\ \text { C } & -3.82786300 & 5.99966800 & 1.60194600 \\ \text { H } & -2.06580700 & 5.05749600 & 0.82560900 \\ \text { H } & -1.55168700 & 5.59910400 & 3.17004500 \\ \text { H } & -1.40278600 & 3.84446300 & 2.90383200 \\ \text { H } & -2.83697500 & 4.50154200 & 3.73760800 \\ \text { H } & -3.32126400 & 6.94215300 & 1.86820300 \\ \text { H } & -4.62807400 & 5.82497400 & 2.34081500 \\ \text { H } & -4.30803400 & 6.14199900 & 0.61924200 \\ \text { C } & -2.22986900 & 3.25931900 & -2.42516400 \\ \text { H } & -2.64680900 & 2.55226800 & -3.14986400 \\ \text { H } & -1.16541900 & 3.17891100 & -2.19556600 \\ \text { C } & -2.97348100 & 4.26939100 & -1.92037300 \\ \text { H } & -2.48674300 & 4.96192700 & -1.22229300 \\ \text { C } & -4.38000400 & 4.60634200 & -2.30343800 \\ \text { H } & -5.02102200 & 4.72598800 & -1.41600300 \\ \text { H } & -4.37974400 & 5.57904900 & -2.82611800 \\ \text { H } & -4.82705800 & 3.84881000 & -2.96061000\end{array}$

$\begin{array}{lrrr}\text { MgCl }_{2} \text {-Ti-isobutyl-Propylene-1,2-re-insertion-TS } & \text { - } \\ \text { Cl } & 8.18859900 & 0.07313400 & -1.45733800 \\ \text { Mg } & 7.50476400 & -1.76979300 & 0.09632100 \\ \text { Cl } & 8.87330400 & 2.32096200 & 1.05850900 \\ \text { Mg } & 6.80955300 & 1.78662000 & -0.25791800 \\ \text { Cl } & 5.44101500 & -2.30413700 & -1.22010500 \\ \text { Cl } & 1.99823600 & -1.12497100 & -1.33712300 \\ \text { Cl } & 4.74580600 & 1.25227800 & -1.57434400 \\ \text { Cl } & 3.37813900 & -2.43357300 & 1.53297500 \\ \text { Mg } & 1.31445700 & -2.96790400 & 0.21660500 \\ \text { Cl } & 6.12572100 & -0.05630600 & 1.29574200 \\ \text { Mg } & 4.06199300 & -0.59059400 & -0.02074000 \\ \text { Cl } & 2.68292200 & 1.12288300 & 1.17872900 \\ \text { Mg } & 0.61858600 & 0.58799200 & -0.13775200 \\ \text { Cl } & -0.74929200 & -3.50221000 & -1.09987200 \\ \text { Cl } & -4.19214400 & -2.32307300 & -1.21697400 \\ \text { Cl } & -1.45592600 & 0.09246700 & -1.52385000\end{array}$




$\begin{array}{lrrr}\text { Cl } & -2.81235000 & -3.63170000 & 1.65314700 \\ \text { Mg } & -4.87599500 & -4.16604000 & 0.33676500 \\ \text { Cl } & -0.06470200 & -1.25435000 & 1.41611900 \\ \text { Mg } & -2.12816200 & -1.78864200 & 0.09947400 \\ \text { Cl } & -3.43097200 & 0.01694400 & 1.32618900 \\ \text { Mg } & -5.57079600 & -0.60952700 & -0.01732600 \\ \text { Cl } & -7.63494700 & -1.14396600 & -1.33388400 \\ \text { Cl } & -6.25505000 & -2.45255200 & 1.53617900 \\ \text { Ti } & -2.83335400 & 2.04901500 & -0.29767200 \\ \text { Cl } & -5.08101100 & 1.51692000 & -1.13074700 \\ \text { Cl } & -0.62744600 & 2.49621800 & 0.70051900 \\ \text { C } & -3.63506700 & 3.84187300 & 0.60942600 \\ \text { H } & -4.68255700 & 4.09892000 & 0.39787900 \\ \text { H } & -3.71005800 & 2.88663000 & 1.21949400 \\ \text { C } & -2.92320500 & 4.89949800 & 1.45869400 \\ \text { C } & -3.03259200 & 4.54591900 & 2.95228900 \\ \text { C } & -3.45762800 & 6.31193700 & 1.19117800 \\ \text { H } & -1.84909000 & 4.87992900 & 1.20682100 \\ \text { H } & -2.51964700 & 5.29951700 & 3.57121100 \\ \text { H } & -2.56850100 & 3.56871600 & 3.15996200 \\ \text { H } & -4.08678400 & 4.50388300 & 3.27474400 \\ \text { H } & -2.93116200 & 7.05062600 & 1.81575200 \\ \text { H } & -4.53309500 & 6.37633200 & 1.42926200 \\ \text { H } & -3.32815700 & 6.61950100 & 0.14214000 \\ \text { C } & -2.34509000 & 3.14442500 & -2.14367100 \\ \text { H } & -2.88185000 & 2.60148600 & -2.92940700 \\ \text { H } & -1.25516900 & 3.05743400 & -2.14466100 \\ \text { C } & -2.93750500 & 4.26683600 & -1.54888100 \\ \text { H } & -2.26214900 & 4.97873000 & -1.06736700 \\ \text { C } & -4.23673900 & 4.82214100 & -2.06822000 \\ \text { H } & -4.71159600 & 5.52747800 & -1.37467200 \\ \text { H } & -4.01336800 & 5.36804900 & -3.00142600 \\ \text { H } & -4.94715400 & 4.01885400 & -2.30682400 \\ & -200 & \end{array}$

$\mathrm{MgCl}_{2}$-Ti-isobutyl-Propylene-1,2-si-coordination

$\begin{array}{lrrr}\text { Cl } & 8.29052500 & 0.16316000 & -1.33139300 \\ \mathrm{Mg} & 7.58041700 & -1.69260400 & 0.19495900 \\ \mathrm{Cl} & 8.92630000 & 2.39186100 & 1.21413600 \\ \mathrm{Mg} & 6.88769600 & 1.86577000 & -0.14412900 \\ \mathrm{Cl} & 5.54181600 & -2.21869600 & -1.16330600 \\ \mathrm{Cl} & 2.10039600 & -1.04215800 & -1.33431900 \\ \mathrm{Cl} & 4.84909400 & 1.33968100 & -1.50239400 \\ \mathrm{Cl} & 3.42888000 & -2.37184900 & 1.55031000 \\ \mathrm{Mg} & 1.39029400 & -2.89793200 & 0.19205000\end{array}$




\begin{tabular}{|c|c|c|c|}
\hline $\mathrm{Cl}$ & 6.17758900 & 0.01000600 & 1.38222300 \\
\hline Mg & 4.13899600 & -0.51606000 & 0.02393300 \\
\hline $\mathrm{Cl}$ & 2.73615100 & 1.18651800 & 1.2112330 \\
\hline Mg & 0.69726100 & 0.66024000 & -0.1471930 \\
\hline $\mathrm{Cl}$ & -0.64825600 & -3.42396900 & $-1.166231 e$ \\
\hline $\mathrm{Cl}$ & -4.08975100 & -2.24746300 & $-1.337317 €$ \\
\hline $\mathrm{Cl}$ & -1.35938700 & 0.15785300 & $-1.542004 \ell$ \\
\hline $\mathrm{Cl}$ & -2.76136400 & -3.57720200 & 1.547323 \\
\hline Mg & -4.79986000 & -4.10328000 & 0.189118 \\
\hline $\mathrm{Cl}$ & -0.01258200 & -1.19529500 & 1.379390 \\
\hline Mg & -2.05107100 & -1.72141500 & 0.021236 \\
\hline $\mathrm{Cl}$ & -3.43239500 & 0.05518300 & 1.258227 \\
\hline Mg & -5.49232000 & -0.54487900 & -0.149943 \\
\hline $\mathrm{Cl}$ & -7.53119600 & -1.07099500 & $-1.508213 t$ \\
\hline $\mathrm{Cl}$ & -6.20270300 & -2.40064700 & 1.376404 \\
\hline $\mathrm{Ti}$ & -2.83590500 & 2.11824100 & -0.1841326 \\
\hline $\mathrm{Cl}$ & -4.84072000 & 1.52236400 & -1.372320 \\
\hline $\mathrm{Cl}$ & -0.48195200 & 2.67415000 & 0.4855780 \\
\hline C & -3.40940100 & 3.35020600 & 1.38275 \\
\hline $\mathrm{H}$ & -2.96558300 & 4.35870000 & 1.3105596 \\
\hline $\mathrm{H}$ & -2.86389500 & 2.83160100 & 2.1962460 \\
\hline C & -4.91812300 & 3.40143100 & 1.685679 \\
\hline C & -5.15896500 & 3.76355700 & 3.1607770 \\
\hline $\mathrm{H}$ & -6.23663500 & 3.78775700 & 3.3942540 \\
\hline $\mathrm{H}$ & -4.73937800 & 4.75820800 & 3.38783806 \\
\hline $\mathrm{H}$ & -4.68030400 & 3.03489600 & 3.8339590 \\
\hline C & -2.15606600 & 3.34473800 & -2.3482226 \\
\hline H & -2.56509400 & 2.52003200 & -2.9396860 \\
\hline $\mathrm{H}$ & -1.06861300 & 3.40534900 & -2.254145 \\
\hline C & -2.95795000 & 4.32071100 & -1.865356 \\
\hline $\mathrm{H}$ & -4.03637700 & 4.24062500 & $-2.045691 €$ \\
\hline C & -2.47456200 & 5.56740000 & -1.191060 \\
\hline $\mathrm{H}$ & -3.14414900 & 5.86925000 & -0.373530 \\
\hline $\mathrm{H}$ & -1.45335200 & 5.44998000 & -0.7998420 \\
\hline $\mathrm{H}$ & -2.47440400 & 6.39264700 & -1.9253406 \\
\hline $\mathrm{H}$ & -5.34252500 & 2.39150000 & $1.527174 €$ \\
\hline C & -5.66210500 & 4.36908600 & $0.759191 €$ \\
\hline $\mathrm{H}$ & -5.55786000 & 4.07743700 & -0.2958960 \\
\hline H & -6.73890700 & 4. 39156100 & 0.989784 \\
\hline & -5.27450100 & 5.39577000 & 0.87775 \\
\hline
\end{tabular}

$\mathrm{MgCl}_{2}$-Ti-isobutyl-Propylene-1,2-si-insertion-TS

$\begin{array}{lrrr}\text { Cl } & 8.25889000 & 0.25745500 & -1.37221900 \\ \text { Mg } & 7.59164700 & -1.65246100 & 0.10591100\end{array}$




\begin{tabular}{|c|c|c|c|}
\hline $\mathrm{Cl}$ & 8.89629800 & 2.41742000 & 1.23149000 \\
\hline Mg & 6.84842700 & 1.90483700 & -0.11797200 \\
\hline $\mathrm{Cl}$ & 5.54377900 & -2.16504600 & -1.24355100 \\
\hline $\mathrm{Cl}$ & 2.08538900 & -1.03025700 & -1.33881700 \\
\hline $\mathrm{Cl}$ & 4.80055900 & 1.39225500 & -1.46743300 \\
\hline $\mathrm{Cl}$ & 3.46607500 & -2.42758000 & 1.48882700 \\
\hline Mg & 1.41822300 & -2.94015400 & 0.13936800 \\
\hline $\mathrm{Cl}$ & 6.18118500 & -0.00508000 & 1.36015800 \\
\hline Mg & 4.13332200 & -0.51764600 & 0.01068000 \\
\hline $\mathrm{Cl}$ & 2.72284000 & 1.12971200 & 1.26496600 \\
\hline Mg & 0.67490400 & 0.61702400 & -0.08463900 \\
\hline $\mathrm{Cl}$ & -0.62959200 & -3.45268500 & -1.21010200 \\
\hline $\mathrm{Cl}$ & -4.08798100 & -2.31789400 & -1.30542600 \\
\hline $\mathrm{Cl}$ & -1.37989400 & 0.14280400 & -1.50168400 \\
\hline $\mathrm{Cl}$ & -2.70746200 & -3.71530300 & 1.52220800 \\
\hline Mg & -4.75523400 & -4.22786700 & 0.17280800 \\
\hline $\mathrm{Cl}$ & 0.00769700 & -1.29274300 & 1.39368200 \\
\hline Mg & -2.04011800 & -1.80534400 & 0.04437300 \\
\hline $\mathrm{Cl}$ & -3.38257800 & -0.06697200 & 1.32888100 \\
\hline Mg & -5.49825700 & -0.67063000 & -0.05107900 \\
\hline $\mathrm{Cl}$ & -7.54633700 & -1.18314900 & -1.40051400 \\
\hline $\mathrm{Cl}$ & -6.16570300 & -2.58046000 & 1.42708700 \\
\hline $\mathrm{Ti}$ & -2.78991300 & 2.02859100 & -0.21275200 \\
\hline $\mathrm{Cl}$ & -5.01791500 & 1.48743100 & -1.10857400 \\
\hline $\mathrm{Cl}$ & -0.60616200 & 2.47446600 & 0.82368500 \\
\hline C & -3.60121000 & 3.77046900 & 0.77642000 \\
\hline $\mathrm{H}$ & -2.88485100 & 4.40193100 & 1.32268300 \\
\hline $\mathrm{H}$ & -3.61645400 & 2.79624700 & 1.36105300 \\
\hline C & -5.02304600 & 4.34001300 & 0.79511900 \\
\hline C & -5.78277400 & 3.81192000 & 2.02503600 \\
\hline $\mathrm{H}$ & -6.81115600 & 4.20651500 & 2.04927800 \\
\hline $\mathrm{H}$ & -5.28209000 & 4.10996600 & 2.96176700 \\
\hline $\mathrm{H}$ & -5.84705700 & 2.71200500 & 2.00686200 \\
\hline C & -2.31956000 & 3.20116600 & -2.02178100 \\
\hline $\mathrm{H}$ & -2.96812100 & 2.68660100 & -2.73760800 \\
\hline $\mathrm{H}$ & -1.24084300 & 3.08966500 & -2.17014900 \\
\hline C & -2.79796000 & 4.31909600 & -1.32774800 \\
\hline $\mathrm{H}$ & -3.83346800 & 4.61216100 & -1.51744300 \\
\hline C & -1.85454300 & 5.40462600 & -0.88107300 \\
\hline $\mathrm{H}$ & -2.29605200 & 6.08080600 & -0.13865700 \\
\hline $\mathrm{H}$ & -0.92910700 & 4.97725300 & -0.47002700 \\
\hline $\mathrm{H}$ & -1.59012100 & 6.00611500 & -1.76813800 \\
\hline $\mathrm{H}$ & -5.55613600 & 3.96120700 & -0.09266900 \\
\hline C & -5.04387300 & 5.87310400 & 0.76405800 \\
\hline
\end{tabular}




$\begin{array}{lllr}\text { H } & -4.58487800 & 6.28267800 & -0.14838300 \\ \text { H } & -6.07945200 & 6.24566700 & 0.80285500 \\ \text { H } & -4.50382500 & 6.29235200 & 1.63023900\end{array}$

\begin{tabular}{|c|c|c|c|}
\hline $\mathrm{Cl}$ & 8.12255200 & -0.17568600 & -1.38596000 \\
\hline Mg & 7.36704700 & -1.94721900 & 0.21677000 \\
\hline $\mathrm{Cl}$ & 8.85110600 & 2.12823500 & 1.06601800 \\
\hline Mg & 6.78503300 & 1.61580400 & -0.25546600 \\
\hline $\mathrm{Cl}$ & 5.30097500 & -2.45965200 & -1.10471400 \\
\hline $\mathrm{Cl}$ & 1.89737700 & -1.18059400 & -1.29571000 \\
\hline $\mathrm{Cl}$ & 4.71896300 & 1.10337400 & -1.57695100 \\
\hline $\mathrm{Cl}$ & 3.20795100 & -2.43969200 & 1.62851000 \\
\hline Mg & 1.14190600 & -2.95212000 & 0.30704800 \\
\hline $\mathrm{Cl}$ & 6.02952800 & -0.15572900 & 1.34726500 \\
\hline Mg & 3.96346400 & -0.66814200 & 0.02576000 \\
\hline $\mathrm{Cl}$ & 2.62592700 & 1.12333500 & 1.15628900 \\
\hline Mg & 0.55967800 & 0.61066600 & -0.16535500 \\
\hline $\mathrm{Cl}$ & -0.92412400 & -3.46451000 & -1.01445300 \\
\hline $\mathrm{Cl}$ & -4.32775800 & -2.18545100 & -1.20553700 \\
\hline $\mathrm{Cl}$ & -1.52769800 & 0.13712800 & -1.51396400 \\
\hline $\mathrm{Cl}$ & -3.01732200 & -3.44460400 & 1.71870900 \\
\hline Mg & -5.08329700 & -3.95703100 & 0.39727200 \\
\hline $\mathrm{Cl}$ & -0.19571300 & -1.16056400 & 1.43763800 \\
\hline Mg & -2.26157900 & -1.67296100 & 0.11603800 \\
\hline $\mathrm{Cl}$ & -3.57365100 & 0.19303300 & 1.30880000 \\
\hline Mg & -5.66514800 & -0.39406500 & -0.07481100 \\
\hline $\mathrm{Cl}$ & -7.73136900 & -0.90643200 & -1.39644700 \\
\hline $\mathrm{Cl}$ & -6.42081200 & -2.16553600 & 1.52777300 \\
\hline $\mathrm{Ti}$ & -2.91047300 & 2.19668900 & -0.18999300 \\
\hline $\mathrm{Cl}$ & -4.93588400 & 1.59029000 & -1.37696000 \\
\hline $\mathrm{Cl}$ & -0.56924700 & 2.64278100 & 0.48651400 \\
\hline C & -3.52388600 & 3.59686800 & 1.18837100 \\
\hline $\mathrm{H}$ & -4.49428700 & 3.74899400 & 0.66405500 \\
\hline $\mathrm{H}$ & -3.74177700 & 2.99975300 & 2.09389200 \\
\hline C & -2.82618300 & 4.92248100 & 1.53603200 \\
\hline C & -2.07916100 & 4.81032500 & 2.87006200 \\
\hline C & -3.81761500 & 6.09529200 & 1.54759400 \\
\hline $\mathrm{H}$ & -2.06731200 & 5.14087100 & 0.76166700 \\
\hline $\mathrm{H}$ & -1.51499700 & 5.73153500 & 3.08792900 \\
\hline H & -1.36780700 & 3.97144100 & 2.85743700 \\
\hline H & -2.78968200 & 4.64496500 & 3.69821000 \\
\hline $\mathrm{H}$ & -3.30906500 & 7.04473000 & 1.78425400 \\
\hline $\mathrm{H}$ & -4.60428000 & 5.93504900 & 2.30418100 \\
\hline
\end{tabular}




$\begin{array}{llll}\text { H } & -4.31436300 & 6.21274300 & 0.56989700 \\ \mathrm{C} & -2.14796300 & 3.26969800 & -2.65844500 \\ \text { H } & -2.68953400 & 2.49496000 & -3.21689300 \\ \mathrm{C} & -2.87087400 & 4.14821100 & -1.92803500 \\ \mathrm{H} & -2.38185100 & 4.95704700 & -1.37644800 \\ \mathrm{H} & -3.96318000 & 4.14721600 & -1.95863100 \\ \mathrm{C} & -0.66575800 & 3.32071400 & -2.85719900 \\ \mathrm{H} & -0.21934200 & 2.31772500 & -2.78044300 \\ \mathrm{H} & -0.45847800 & 3.67709200 & -3.88161100 \\ \mathrm{H} & -0.17387400 & 3.99267800 & -2.14096900\end{array}$

$\mathrm{MgCl}_{2}$-Ti-isobutyl-Propylene-2,1-re-insertion-TS

$\begin{array}{lrrr}\text { Cl } & 8.13553000 & -0.08781500 & -1.40259600 \\ \mathrm{Mg} & 7.40140400 & -1.90206500 & 0.16182600 \\ \mathrm{Cl} & 8.85476900 & 2.16518200 & 1.09895100 \\ \mathrm{Mg} & 6.78768900 & 1.66582700 & -0.22595900 \\ \mathrm{Cl} & 5.33432400 & -2.40142300 & -1.16308400 \\ \mathrm{Cl} & 1.91937500 & -1.14714600 & -1.31137300 \\ \mathrm{Cl} & 4.72061100 & 1.16647300 & -1.55087000 \\ \mathrm{Cl} & 3.25235700 & -2.46202800 & 1.57797400 \\ \mathrm{Mg} & 1.18533000 & -2.96139100 & 0.25313100 \\ \mathrm{Cl} & 6.05356300 & -0.14842300 & 1.33846400 \\ \mathrm{Mg} & 3.98650300 & -0.64773500 & 0.01350500 \\ \mathrm{Cl} & 2.63865600 & 1.10590100 & 1.19015500 \\ \mathrm{Mg} & 0.57102100 & 0.60610800 & -0.13471500 \\ \mathrm{Cl} & -0.88173800 & -3.46069900 & -1.07183900 \\ \mathrm{Cl} & -4.29669700 & -2.20641000 & -1.22018200 \\ \mathrm{Cl} & -1.50467900 & 0.14269800 & -1.51919000 \\ \mathrm{Cl} & -2.96390000 & -3.52132500 & 1.66917500 \\ \mathrm{Mg} & -5.03085100 & -4.02069500 & 0.34430600 \\ \mathrm{Cl} & -0.16261300 & -1.20762900 & 1.42987900 \\ \mathrm{Mg} & -2.22923000 & -1.70708300 & 0.10452500 \\ \mathrm{Cl} & -3.50376100 & 0.12884100 & 1.31281600 \\ \mathrm{Mg} & -5.64435000 & -0.45268700 & -0.04326000 \\ \mathrm{Cl} & -7.71162200 & -0.95218000 & -1.36841700 \\ \mathrm{Cl} & -6.37871700 & -2.26707300 & 1.52090600 \\ \mathrm{Ti} & -2.82404200 & 2.13363700 & -0.30970700 \\ \mathrm{Cl} & -5.05987600 & 1.62735700 & -1.20923600 \\ \mathrm{Cl} & -0.66326400 & 2.49540200 & 0.78022500 \\ \mathrm{C} & -3.62811300 & 3.92470000 & 0.56990700 \\ \mathrm{H} & -4.67147000 & 4.19580700 & 0.35220700 \\ \mathrm{H} & -3.71246600 & 2.98179800 & 1.19885800 \\ \mathrm{C} & -2.88750600 & 5.00572400 & 1.36368200 \\ \mathrm{C} & -2.87284900 & 4.66117000 & 2.86246300\end{array}$




$\begin{array}{llll}\mathrm{C} & -3.49494300 & 6.39362500 & 1.12412100 \\ \mathrm{H} & -1.83556900 & 5.01912400 & 1.03043700 \\ \mathrm{H} & -2.33872100 & 5.43578900 & 3.43582500 \\ \mathrm{H} & -2.36513600 & 3.70024400 & 3.03946800 \\ \mathrm{H} & -3.89848600 & 4.59145000 & 3.26286700 \\ \mathrm{H} & -2.91944300 & 7.17055500 & 1.65163900 \\ \mathrm{H} & -4.53321100 & 6.43271800 & 1.49501800 \\ \mathrm{H} & -3.51563800 & 6.66188100 & 0.05579300 \\ \mathrm{C} & -2.28994300 & 3.24021000 & -2.22962000 \\ \mathrm{H} & -2.84050900 & 2.56563400 & -2.89802300 \\ \mathrm{C} & -3.02625500 & 4.24731100 & -1.60921400 \\ \mathrm{H} & -2.51138300 & 5.13960000 & -1.24797000 \\ \mathrm{H} & -4.08837900 & 4.35880400 & -1.83034800 \\ \mathrm{C} & -0.80321500 & 3.37833000 & -2.48647400 \\ \mathrm{H} & -0.30746000 & 2.39970900 & -2.56434400 \\ \mathrm{H} & -0.64084800 & 3.89297600 & -3.44952000 \\ \mathrm{H} & -0.30497400 & 3.95916000 & -1.69770100\end{array}$

$\mathrm{MgCl}_{2}$-Ti-isobutyl-Propylene-2,1-si-coordination

$\begin{array}{lrrr}\text { Cl } & -8.16307300 & -0.20214000 & 1.40909900 \\ \text { Mg } & -7.38915000 & -1.91274500 & -0.25003700 \\ \text { Cl } & -8.90657000 & 2.17592500 & -0.96641300 \\ \text { Mg } & -6.83879100 & 1.63739900 & 0.34196200 \\ \text { Cl } & -5.32137200 & -2.45127200 & 1.05833800 \\ \text { Cl } & -1.92930800 & -1.15025800 & 1.29958100 \\ \text { Cl } & -4.77101600 & 1.09887500 & 1.65033800 \\ \text { Cl } & -3.22316600 & -2.32233500 & -1.66793600 \\ \text { Mg } & -1.15541800 & -2.86085600 & -0.35958600 \\ \text { Cl } & -6.06486800 & -0.07320600 & -1.31717600 \\ \text { Mg } & -3.99709700 & -0.61171500 & -0.00878100 \\ \text { Cl } & -2.67279600 & 1.22781500 & -1.07595500 \\ \text { Mg } & -0.60484200 & 0.68905100 & 0.23258900 \\ \text { Cl } & 0.91232100 & -3.39934300 & 0.94881000 \\ \text { Cl } & 4.30442700 & -2.09833300 & 1.19014300 \\ \text { Cl } & 1.50714100 & 0.17812000 & 1.56899100 \\ \text { Cl } & 3.01070000 & -3.27046100 & -1.77740200 \\ \text { Mg } & 5.07836300 & -3.80897400 & -0.46909200 \\ \text { Cl } & 0.16896700 & -1.02124600 & -1.42681400 \\ \text { Mg } & 2.23652700 & -1.55975000 & -0.11831500 \\ \text { Cl } & 3.50467300 & 0.32976700 & -1.24891000 \\ \text { Mg } & 5.62854800 & -0.25889800 & 0.12274400 \\ \text { Cl } & 7.69650800 & -0.79734700 & 1.43129000 \\ \text { Cl } & 6.40270500 & -1.96943900 & -1.53619300 \\ \text { Ti } & 2.85942500 & 2.30580200 & 0.27422000\end{array}$




$\begin{array}{lrrr}\text { Cl } & 4.99473400 & 1.81526200 & 1.30318500 \\ \text { Cl } & 0.44365700 & 2.82661200 & -0.14020000 \\ \text { C } & 3.42320400 & 3.66427300 & -1.17219200 \\ \text { H } & 3.61874200 & 4.53793600 & -0.51367900 \\ \text { H } & 4.41468000 & 3.25251000 & -1.45247400 \\ \text { C } & 2.61425200 & 4.07146300 & -2.40819900 \\ \text { C } & 2.28873000 & 2.87307600 & -3.30717900 \\ \text { C } & 3.34797800 & 5.16716500 & -3.19884800 \\ \text { H } & 1.65862700 & 4.49319000 & -2.05140800 \\ \text { H } & 1.72451900 & 3.19349500 & -4.19744000 \\ \text { H } & 1.68240500 & 2.12461600 & -2.77610100 \\ \text { H } & 3.21077000 & 2.37464600 & -3.65086900 \\ \text { H } & 2.74683000 & 5.50331700 & -4.06006400 \\ \text { H } & 4.31055900 & 4.79151400 & -3.58610400 \\ \text { H } & 3.56022600 & 6.04695400 & -2.56974800 \\ \text { C } & 2.15803200 & 3.18199200 & 2.92135300 \\ \text { C } & 2.68509000 & 4.14139700 & 2.13057500 \\ \text { H } & 2.04505200 & 4.71016100 & 1.44845800 \\ \text { H } & 3.73064500 & 4.44896900 & 2.23034800 \\ \text { H } & 1.09373400 & 2.94786000 & 2.79772400 \\ \text { C } & 2.88815500 & 2.42722000 & 3.98379300 \\ \text { H } & 2.44460700 & 2.66755600 & 4.96597300 \\ \text { H } & 2.76880100 & 1.34226700 & 3.83877800 \\ \text { H } & 3.95983100 & 2.66681000 & 4.00297800\end{array}$

$\mathrm{MgCl}_{2}$-Ti-isobutyl-Propylene-2,1-si-insertion-TS

$\begin{array}{lrrr}\text { Cl } & -8.16989900 & 0.00022500 & 1.43767800 \\ \text { Mg } & -7.46358800 & -1.83199300 & -0.11858700 \\ \text { Cl } & -8.88709300 & 2.24128400 & -1.07515300 \\ \text { Mg } & -6.81496300 & 1.73293700 & 0.23840000 \\ \text { Cl } & -5.39145900 & -2.34034300 & 1.19496800 \\ \text { Cl } & -1.96403600 & -1.11623100 & 1.30928200 \\ \text { Cl } & -4.74283700 & 1.22459000 & 1.55195600 \\ \text { Cl } & -3.33021100 & -2.43984600 & -1.56057500 \\ \text { Mg } & -1.25825300 & -2.94820900 & -0.24724000 \\ \text { Cl } & -6.10865200 & -0.09928200 & -1.31786600 \\ \text { Mg } & -4.03658500 & -0.60747000 & -0.00415200 \\ \text { Cl } & -2.68181000 & 1.12541200 & -1.20311100 \\ \text { Mg } & -0.60914000 & 0.61699700 & 0.11056500 \\ \text { Cl } & 0.81393600 & -3.45631500 & 1.06674700 \\ \text { Cl } & 4.24080900 & -2.23165500 & 1.18082700 \\ \text { Cl } & 1.47198500 & 0.13613800 & 1.49776600 \\ \text { Cl } & 2.87551700 & -3.55621900 & -1.68870600 \\ \text { Mg } & 4.94745400 & -4.06451800 & -0.37529900\end{array}$




$\begin{array}{lrrr}\text { Cl } & 0.09656200 & -1.21579600 & -1.44706600 \\ \text { Mg } & 2.16924900 & -1.72462700 & -0.13408200 \\ \text { Cl } & 3.43778700 & 0.10336700 & -1.34874400 \\ \text { Mg } & 5.59580900 & -0.49931700 & -0.01862400 \\ \text { Cl } & 7.66803300 & -1.00768100 & 1.29535800 \\ \text { Cl } & 6.30267000 & -2.33178500 & -1.57446200 \\ \text { Ti } & 2.83182100 & 2.12362200 & 0.29175100 \\ \text { Cl } & 5.11172200 & 1.64243800 & 1.06334200 \\ \text { Cl } & 0.61412100 & 2.54951400 & -0.69967100 \\ \text { C } & 3.60333000 & 3.92683000 & -0.60114600 \\ \text { H } & 4.63811300 & 4.18060200 & -0.32657100 \\ \text { H } & 3.69150000 & 2.97568600 & -1.21760900 \\ \text { C } & 2.94536900 & 5.00116600 & -1.46689600 \\ \text { C } & 3.19464300 & 4.70801500 & -2.95690100 \\ \text { C } & 3.43601700 & 6.40680200 & -1.09763700 \\ \text { H } & 1.85376800 & 4.95226800 & -1.31076300 \\ \text { H } & 2.72747800 & 5.48015000 & -3.58910100 \\ \text { H } & 2.76834500 & 3.73394600 & -3.24540200 \\ \text { H } & 4.27412300 & 4.69335300 & -3.18355900 \\ \text { H } & 2.92688300 & 7.17016600 & -1.70670200 \\ \text { H } & 4.52031200 & 6.50020500 & -1.27886100 \\ \text { H } & 3.25324400 & 6.65067200 & -0.03992900 \\ \text { C } & 2.28252300 & 3.21702100 & 2.19593300 \\ \text { C } & 2.70011400 & 4.31535100 & 1.44548400 \\ \text { H } & 1.95732700 & 4.92160000 & 0.92556000 \\ \text { H } & 3.63983100 & 4.81239300 & 1.69711500 \\ \text { H } & 1.20863400 & 2.99607000 & 2.16357400 \\ \text { C } & 3.03136300 & 2.68900900 & 3.40005000 \\ \text { H } & 2.59311500 & 3.11804300 & 4.31841300 \\ \text { H } & 2.95976100 & 1.59501100 & 3.48175600 \\ \text { H } & 4.09608200 & 2.95653100 & 3.36479600\end{array}$

$\mathrm{MgCl}_{2}$-Ti-isobutyl-DiBS-Mg2-C-Propylene-1, 2-re-coordination

$\begin{array}{lrrr}\text { Cl } & 5.94460100 & -3.23880200 & -1.32271400 \\ \text { Mg } & 4.68522800 & -4.71756200 & 0.26024500 \\ \text { Cl } & 7.29621100 & -1.26528200 & 1.15266400 \\ \text { Mg } & 5.18463500 & -1.14016800 & -0.18775500 \\ \text { Cl } & 2.57220200 & -4.59026100 & -1.08035000 \\ \text { Cl } & -0.30063700 & -2.36279900 & -1.28552100 \\ \text { Cl } & 3.07273200 & -1.01128900 & -1.52714200 \\ \text { Cl } & 0.55297500 & -3.96893200 & 1.63779700 \\ \text { Mg } & -1.55987700 & -3.84164900 & 0.29735300 \\ \text { Cl } & 3.92518000 & -2.61749200 & 1.39546700 \\ \text { Mg } & 1.81230400 & -2.49023100 & 0.05478900\end{array}$




\begin{tabular}{|c|c|c|c|}
\hline $\mathrm{Cl}$ & 1.05233600 & -0.39019800 & 1.19022600 \\
\hline Mg & -1.06120700 & -0.26302600 & -0.15051100 \\
\hline $\mathrm{Cl}$ & -3.67277600 & -3.71430200 & -1.04324106 \\
\hline $\mathrm{Cl}$ & -6.54576800 & -1.48698300 & $-1.248633 e$ \\
\hline $\mathrm{Cl}$ & -3.19566600 & -0.06468100 & $-1.508101 €$ \\
\hline $\mathrm{Cl}$ & -5.69231100 & -3.09298600 & 1.6747600 \\
\hline Mg & -7.80512000 & -2.96569800 & 0.3343210 \\
\hline $\mathrm{Cl}$ & -2.31997000 & -1.74158000 & 1.4325820 \\
\hline Mg & -4.43280400 & -1.61443800 & 0.0921310 \\
\hline $\mathrm{Cl}$ & -5.14130000 & 0.54130700 & 1.2962860 \\
\hline Mg & -7.30538100 & 0.61296400 & -0.1133190 \\
\hline $\mathrm{Cl}$ & -9.41873000 & 0.74031200 & -1.4538440 \\
\hline $\mathrm{Cl}$ & -8.56513400 & -0.86564500 & 1.4695580 \\
\hline $\mathrm{Ti}$ & -3.90363800 & 2.27817500 & -0.1750070 \\
\hline $\mathrm{Cl}$ & -6.03548300 & 2.37296700 & -1.3126750 \\
\hline $\mathrm{Cl}$ & -1.48944200 & 2.04026300 & 0.4380390 \\
\hline C & 5.65384500 & 2.56823200 & 0.50826300 \\
\hline $\mathrm{H}$ & 5.37499000 & 3.58590400 & 0.81590206 \\
\hline C & 5.45817000 & 2.51297800 & -1.03663700 \\
\hline $\mathrm{H}$ & 5.91435100 & 3.43441800 & -1.42404100 \\
\hline C & 4.72470700 & 1.63483800 & 1.26203006 \\
\hline C & 6.24934700 & 1.37730600 & -1.65876300 \\
\hline 0 & 7.27123500 & 1.78966100 & -2.38693600 \\
\hline 0 & 4.12806700 & 2.23963200 & 2.27352306 \\
\hline C & 8.15923400 & 0.77603500 & -2.94252200 \\
\hline $\mathrm{H}$ & 7.54460700 & -0.03867800 & -3.35235906 \\
\hline $\mathrm{H}$ & 8.66686000 & 1.29932300 & $-3.7643640 e$ \\
\hline C & 9.13481500 & 0.26583200 & -1.89684900 \\
\hline $\mathrm{H}$ & 9.70772700 & 1.09962900 & -1.46196800 \\
\hline $\mathrm{H}$ & 8.62120600 & -0.27643100 & -1.08709006 \\
\hline $\mathrm{H}$ & 9.84505500 & -0.42685700 & -2.37607900 \\
\hline C & 3.25737000 & 1.49383100 & 3.16869200 \\
\hline $\mathrm{H}$ & 3.10573700 & 0.48506800 & 2.76651700 \\
\hline H & 3.80046800 & 1.42599300 & 4.12429400 \\
\hline C & 1.95246900 & 2.24902900 & 3.30650300 \\
\hline $\mathrm{H}$ & 1.42977600 & 2.29264400 & 2.34015000 \\
\hline $\mathrm{H}$ & 1.29644100 & 1.72052100 & 4.01516100 \\
\hline 0 & 4.55514100 & 0.45430000 & 1.00104100 \\
\hline 0 & 6.00095100 & 0.18890300 & -1.52010400 \\
\hline C & 7.11578600 & 2.28701800 & 0.92547200 \\
\hline $\mathrm{H}$ & 7.75734600 & 3.02016200 & 0.40471700 \\
\hline $\mathrm{H}$ & 7.39485900 & 1.28392700 & 0.56853700 \\
\hline C & 7.41035700 & 2.31946600 & 2.43428906 \\
\hline $\mathrm{H}$ & 6.77372500 & 1.55193900 & 2.9108100 \\
\hline
\end{tabular}




\begin{tabular}{|c|c|c|c|}
\hline C & 8.86310600 & 1.89049800 & 2.67109900 \\
\hline $\mathrm{H}$ & 9.10381000 & 1.88228500 & 3.74664800 \\
\hline $\mathrm{H}$ & 9.02867400 & 0.87734100 & 2.27334400 \\
\hline $\mathrm{H}$ & 9.56551400 & 2.58463100 & 2.17639300 \\
\hline C & 7.10263800 & 3.67757800 & 3.07483900 \\
\hline $\mathrm{H}$ & 6.03443700 & 3.93364400 & 3.00262300 \\
\hline $\mathrm{H}$ & 7.36953800 & 3.67647400 & 4.14422200 \\
\hline $\mathrm{H}$ & 7.68389200 & 4.48144600 & 2.58932700 \\
\hline C & 3.96995100 & 2.46507700 & -1.43659300 \\
\hline $\mathrm{H}$ & 3.49046900 & 1.60584700 & -0.94735900 \\
\hline $\mathrm{H}$ & 3.49370900 & 3.37648300 & -1.03130200 \\
\hline C & 3.67583300 & 2.36028800 & -2.94196500 \\
\hline $\mathrm{H}$ & 4.10540400 & 1.40455500 & -3.29364700 \\
\hline C & 4.29732900 & 3.50284300 & -3.75388200 \\
\hline $\mathrm{H}$ & 4.02811200 & 3.42067300 & -4.81931900 \\
\hline $\mathrm{H}$ & 3.93746800 & 4.48315700 & -3.39442100 \\
\hline $\mathrm{H}$ & 5.39720100 & 3.50239300 & -3.69359300 \\
\hline C & 2.15966400 & 2.27244200 & -3.15772500 \\
\hline $\mathrm{H}$ & 1.65661800 & 3.18556300 & -2.79257000 \\
\hline $\mathrm{H}$ & 1.91492500 & 2.15747500 & -4.22594100 \\
\hline $\mathrm{H}$ & 1.74583700 & 1.40584500 & -2.61867800 \\
\hline $\mathrm{H}$ & 2.12103300 & 3.27230200 & 3.67664500 \\
\hline C & -4.03538900 & 3.75812400 & 1.25796300 \\
\hline $\mathrm{H}$ & -4.91927400 & 4.22109100 & 0.76459900 \\
\hline $\mathrm{H}$ & -4.42302500 & 3.20499100 & 2.13478200 \\
\hline C & -2.97851100 & 4.79349300 & 1.67528000 \\
\hline C & -2.27771200 & 4.36649900 & 2.97084900 \\
\hline C & -3.58703800 & 6.19731100 & 1.81359700 \\
\hline $\mathrm{H}$ & -2.20004300 & 4.84618500 & 0.89154800 \\
\hline $\mathrm{H}$ & -1.46605600 & 5.06499500 & 3.23135900 \\
\hline $\mathrm{H}$ & -1.84346500 & 3.36077100 & 2.87623600 \\
\hline $\mathrm{H}$ & -2.99412200 & 4.35311700 & 3.81023000 \\
\hline $\mathrm{H}$ & -2.82208900 & 6.93706900 & 2.10288600 \\
\hline $\mathrm{H}$ & -4.37540700 & 6.20628300 & 2.58497600 \\
\hline $\mathrm{H}$ & -4.04205100 & 6.53641600 & 0.86811700 \\
\hline C & -2.91307900 & 3.15809400 & -2.44894900 \\
\hline $\mathrm{H}$ & -3.58621800 & 2.68844800 & -3.17337800 \\
\hline $\mathrm{H}$ & -1.95064000 & 2.67532900 & -2.26819900 \\
\hline C & -3.21226500 & 4.34624800 & -1.87839700 \\
\hline $\mathrm{H}$ & -2.48085000 & 4.77545400 & -1.18182600 \\
\hline C & -4.40410000 & 5.19712600 & -2.18883700 \\
\hline $\mathrm{H}$ & -4.91085200 & 5.53201600 & -1.27056800 \\
\hline $\mathrm{H}$ & -4.06182000 & 6.10852200 & -2.71007500 \\
\hline $\mathrm{H}$ & -5.13282300 & 4.67697100 & -2.82453600 \\
\hline
\end{tabular}




\begin{tabular}{|c|c|c|c|}
\hline $\mathrm{Cl}$ & 6.02110400 & -3.17458600 & -1.65983700 \\
\hline Mg & 4.82403900 & -4.69703100 & -0.06995800 \\
\hline $\mathrm{Cl}$ & 7.43863500 & -1.24516300 & 0.81343200 \\
\hline Mg & 5.28385900 & -1.10702400 & -0.45501500 \\
\hline $\mathrm{Cl}$ & 2.66778800 & -4.55671100 & -1.33849200 \\
\hline $\mathrm{Cl}$ & -0.22556900 & -2.34732000 & -1.40170600 \\
\hline $\mathrm{Cl}$ & 3.12875300 & -0.96514500 & -1.72234200 \\
\hline $\mathrm{Cl}$ & 0.73358100 & -4.01014100 & 1.45646000 \\
\hline Mg & -1.42252800 & -3.86982900 & 0.18802600 \\
\hline $\mathrm{Cl}$ & 4.08669900 & -2.62803500 & 1.13515400 \\
\hline Mg & 1.93058200 & -2.48778500 & -0.13343400 \\
\hline $\mathrm{Cl}$ & 1.19337300 & -0.41882600 & 1.07183000 \\
\hline Mg & -0.96332000 & -0.27871800 & -0.19674800 \\
\hline $\mathrm{Cl}$ & -3.57859700 & -3.72948100 & -1.08044400 \\
\hline $\mathrm{Cl}$ & -6.47212500 & -1.52024000 & -1.14387900 \\
\hline $\mathrm{Cl}$ & -3.11422200 & -0.10171300 & -1.53810300 \\
\hline $\mathrm{Cl}$ & -5.51311500 & -3.18292100 & 1.71436300 \\
\hline Mg & -7.66913900 & -3.04262600 & 0.44597300 \\
\hline $\mathrm{Cl}$ & -2.15986900 & -1.80086400 & 1.39317800 \\
\hline Mg & -4.31583900 & -1.66033700 & 0.12474600 \\
\hline $\mathrm{Cl}$ & -4.94851600 & 0.48138700 & 1.34854800 \\
\hline Mg & -7.20915000 & 0.54852100 & 0.06129400 \\
\hline $\mathrm{Cl}$ & -9.36558400 & 0.68901700 & -1.20715900 \\
\hline $\mathrm{Cl}$ & -8.40648100 & -0.97363300 & 1.65108300 \\
\hline $\mathrm{Ti}$ & -3.75576700 & 2.19315400 & -0.33319400 \\
\hline $\mathrm{Cl}$ & -6.07362700 & 2.38095900 & -1.12049900 \\
\hline $\mathrm{Cl}$ & -1.51056800 & 1.93128700 & 0.65326600 \\
\hline C & 5.36810900 & 2.52354700 & 0.78834800 \\
\hline $\mathrm{H}$ & 5.02941100 & 3.43873700 & 1.29399000 \\
\hline C & 4.92330700 & 2.64615000 & -0.70492500 \\
\hline H & 5.17033300 & 3.67254800 & -1.00918000 \\
\hline C & 4.68684900 & 1.36357200 & 1.48165300 \\
\hline C & 5.78121500 & 1.75467200 & -1.59009700 \\
\hline 0 & 6.60371600 & 2.44217600 & -2.36481600 \\
\hline 0 & 4.19462800 & 1.68126700 & 2.66502700 \\
\hline C & 7.58178900 & 1.68950700 & -3.13867400 \\
\hline $\mathrm{H}$ & 7.06606000 & 0.85376900 & -3.63421800 \\
\hline $\mathrm{H}$ & 7.91955100 & 2.40823300 & -3.89756200 \\
\hline C & 8.72716600 & 1.19964100 & -2.26802600 \\
\hline $\mathrm{H}$ & 9.20158800 & 2.04132200 & -1.74013400 \\
\hline H & 8.39355400 & 0.45608600 & -1.52742200 \\
\hline $\mathrm{H}$ & 9.48675400 & 0.72449800 & -2.90902900 \\
\hline
\end{tabular}




\begin{tabular}{|c|c|c|c|}
\hline C & 3.58784800 & 0.64828400 & 3.49113100 \\
\hline $\mathrm{H}$ & 3.39353000 & -0.23742900 & 2.87373100 \\
\hline $\mathrm{H}$ & 4.33779100 & 0.39089900 & 4.25577200 \\
\hline C & 2.31935800 & 1.20983900 & 4.09491700 \\
\hline $\mathrm{H}$ & 1.59227800 & 1.44243200 & 3.30340500 \\
\hline $\mathrm{H}$ & 1.86601700 & 0.45844600 & 4.76004400 \\
\hline 0 & 4.61402400 & 0.24130900 & 1.01332000 \\
\hline 0 & 5.78863300 & 0.53221200 & -1.59244800 \\
\hline C & 6.89876200 & 2.37511900 & 0.95253500 \\
\hline $\mathrm{H}$ & 7.37554300 & 3.25482500 & 0.48463100 \\
\hline $\mathrm{H}$ & 7.22420900 & 1.48289500 & 0.39759200 \\
\hline C & 7.41514700 & 2.20596900 & 2.39133400 \\
\hline $\mathrm{H}$ & 6.94374800 & 1.29574200 & 2.80389400 \\
\hline C & 8.92317100 & 1.93270600 & 2.35225700 \\
\hline H & 9.32582200 & 1.78985800 & 3.36820300 \\
\hline $\mathrm{H}$ & 9.12661500 & 1.01913600 & 1.77242200 \\
\hline $\mathrm{H}$ & 9.46747200 & 2.77662400 & 1.89222900 \\
\hline C & 7.06994700 & 3.39417400 & 3.29534600 \\
\hline $\mathrm{H}$ & 5.98266800 & 3.51669300 & 3.41636400 \\
\hline $\mathrm{H}$ & 7.49881200 & 3.25883900 & 4.30154700 \\
\hline $\mathrm{H}$ & 7.48025300 & 4.33361600 & 2.88423100 \\
\hline C & 3.40390900 & 2.41682700 & -0.85605600 \\
\hline $\mathrm{H}$ & 3.14278000 & 1.42757300 & -0.46140800 \\
\hline $\mathrm{H}$ & 2.90638800 & 3.15865700 & -0.20448300 \\
\hline C & 2.81078300 & 2.51400800 & -2.26988700 \\
\hline H & 3.29097400 & 1.73196200 & -2.88501300 \\
\hline C & 3.05275800 & 3.87509100 & -2.93127900 \\
\hline H & 2.57996400 & 3.92235300 & -3.92558700 \\
\hline $\mathrm{H}$ & 2.62973400 & 4.69245800 & -2.32077300 \\
\hline H & 4.12665600 & 4.07797400 & -3.07063600 \\
\hline C & 1.31275300 & 2.17253700 & -2.20637400 \\
\hline $\mathrm{H}$ & 0.77112200 & 2.87406500 & -1.54788000 \\
\hline $\mathrm{H}$ & 0.84987900 & 2.21155600 & -3.20580400 \\
\hline H & 1.18014300 & 1.15317600 & -1.80699900 \\
\hline $\mathrm{H}$ & 2.52396100 & 2.11939200 & 4.68088800 \\
\hline C & -3.92417700 & 4.16729800 & 0.53794600 \\
\hline $\mathrm{H}$ & -4.83587100 & 4.74447900 & 0.32889900 \\
\hline $\mathrm{H}$ & -4.29367900 & 3.30134600 & 1.17304700 \\
\hline C & -2.89329200 & 4.95584900 & 1.35218100 \\
\hline C & -3.06184800 & 4.66490100 & 2.85339100 \\
\hline C & -2.96790500 & 6.46217400 & 1.07391700 \\
\hline $\mathrm{H}$ & -1.88722700 & 4.59947400 & 1.07198100 \\
\hline H & -2.32035200 & 5.22390200 & 3.44666100 \\
\hline $\mathrm{H}$ & -2.92151500 & 3.59262600 & 3.0628130 \\
\hline
\end{tabular}




$\begin{array}{llll}\text { H } & -4.06596900 & 4.95730500 & 3.20465200 \\ \text { H } & -2.21860500 & 7.00565300 & 1.67082900 \\ \text { H } & -3.96206300 & 6.86091400 & 1.33852600 \\ \text { H } & -2.78118900 & 6.70337700 & 0.01606400 \\ \text { C } & -2.95777600 & 3.04026100 & -2.20348800 \\ \text { H } & -3.64654400 & 2.67667000 & -2.97412900 \\ \text { H } & -1.94869500 & 2.61797800 & -2.20933000 \\ \text { C } & -3.16357500 & 4.30417700 & -1.63400000 \\ \text { H } & -2.29354200 & 4.78112100 & -1.17560000 \\ \text { C } & -4.23357800 & 5.22413500 & -2.15875300 \\ \text { H } & -4.45761400 & 6.05585700 & -1.47892500 \\ \text { H } & -3.86580200 & 5.65407800 & -3.10668600 \\ \text { H } & -5.16069000 & 4.67470700 & -2.37148800\end{array}$

$\mathrm{MgCl}_{2}$-Ti-isobutyl-DiBS-Mg2-C-Propylene-1,2-si-coordination

$\begin{array}{lrrr}\text { Cl } & 6.10315000 & -3.11973500 & -1.45906000 \\ \text { Mg } & 4.88155600 & -4.62660100 & 0.12699600 \\ \text { Cl } & 7.44642200 & -1.13877500 & 1.01491300 \\ \text { Mg } & 5.32096100 & -1.03944800 & -0.30557100 \\ \text { Cl } & 2.75460900 & -4.52511100 & -1.19364100 \\ \text { Cl } & -0.15441000 & -2.34181800 & -1.36031300 \\ \text { Cl } & 3.19512500 & -0.93636800 & -1.62500100 \\ \text { Cl } & 0.75091700 & -3.95023500 & 1.54613800 \\ \text { Mg } & -1.37589700 & -3.84874500 & 0.22559200 \\ \text { Cl } & 4.09926400 & -2.54487900 & 1.28075700 \\ \text { Mg } & 1.97244400 & -2.44346000 & -0.03992900 \\ \text { Cl } & 1.19026800 & -0.36178200 & 1.11399200 \\ \text { Mg } & -0.93703700 & -0.26035400 & -0.20678100 \\ \text { Cl } & -3.50266300 & -3.74722500 & -1.09497400 \\ \text { Cl } & -6.41182700 & -1.56405400 & -1.26187500 \\ \text { Cl } & -3.07958300 & -0.12385900 & -1.55948800 \\ \text { Cl } & -5.50665600 & -3.17236500 & 1.64466400 \\ \text { Mg } & -7.63338500 & -3.07089700 & 0.32417000 \\ \text { Cl } & -2.15819600 & -1.76703600 & 1.37940100 \\ \text { Mg } & -4.28494100 & -1.66562500 & 0.05899300 \\ \text { Cl } & -5.01842400 & 0.48253200 & 1.26106200 \\ \text { Mg } & -7.19380300 & 0.51750800 & -0.10806100 \\ \text { Cl } & -9.32095300 & 0.61906000 & -1.42859400 \\ \text { Cl } & -8.41565800 & -0.98920200 & 1.47792200 \\ \text { Ti } & -3.82075100 & 2.21460900 & -0.24650200 \\ \text { Cl } & -5.95206700 & 2.27943900 & -1.36542300 \\ \text { Cl } & -1.38147700 & 2.04546500 & 0.35562100 \\ \text { C } & 5.52887000 & 2.63193800 & 0.73152300 \\ \text { H } & 5.19454300 & 3.58803000 & 1.15840700\end{array}$




\begin{tabular}{|c|c|c|c|}
\hline c & 5.21150100 & 2.69288200 & -0.79491200 \\
\hline H & 5.54794800 & 3.68433600 & -1.12825500 \\
\hline c & 4.74375700 & 1.54895500 & 1.44301300 \\
\hline C & 6.06446300 & 1.70112900 & -1.56823500 \\
\hline 0 & 6.99502200 & 2.28297800 & -2.30480200 \\
\hline 0 & 4.20460500 & 1.96984200 & 2.57258700 \\
\hline C & 7.95638300 & 1.41972600 & -2.97885000 \\
\hline $\mathrm{H}$ & 7.40750600 & 0.59827700 & -3.46233800 \\
\hline $\mathrm{H}$ & 8.39101500 & 2.06985500 & -3.75028600 \\
\hline C & 9.00644100 & 0.89571900 & -2.01388900 \\
\hline $\mathrm{H}$ & 9.51612000 & 1.72849700 & -1.50489000 \\
\hline $\mathrm{H}$ & 8.57171500 & 0.22604500 & -1.25497200 \\
\hline $\mathrm{H}$ & 9.76056200 & 0.32592600 & -2.57994100 \\
\hline C & 3.48646100 & 1.03703700 & 3.42763900 \\
\hline $\mathrm{H}$ & 3.32541300 & 0.09919800 & 2.88191200 \\
\hline $\mathrm{H}$ & 4.14959300 & 0.84388400 & 4.28550200 \\
\hline C & 2.18257000 & 1.68245000 & 3.84424300 \\
\hline $\mathrm{H}$ & 1.54440800 & 1.85395800 & 2.96535700 \\
\hline $\mathrm{H}$ & 1.64136800 & 1.00825400 & 4.52606500 \\
\hline 0 & 4.63064400 & 0.40317700 & 1.04160200 \\
\hline 0 & 5.95928400 & 0.48398700 & -1.52546800 \\
\hline C & 7.03757900 & 2.43964100 & 1.01516000 \\
\hline $\mathrm{H}$ & 7.57666500 & 3.27740600 & 0.53793300 \\
\hline $\mathrm{H}$ & 7.36738400 & 1.50969000 & 0.52861000 \\
\hline C & 7.44810300 & 2.33361900 & 2.49343200 \\
\hline $\mathrm{H}$ & 6.91648400 & 1.46448000 & 2.92097400 \\
\hline C & 8.94477000 & 2.01137100 & 2.57448400 \\
\hline $\mathrm{H}$ & 9.27218700 & 1.91197400 & 3.62215100 \\
\hline $\mathrm{H}$ & 9.15620300 & 1.06240800 & 2.05808500 \\
\hline $\mathrm{H}$ & 9.54786200 & 2.81180800 & 2.11005100 \\
\hline C & 7.08644000 & 3.58141300 & 3.30627400 \\
\hline $\mathrm{H}$ & 5.99912800 & 3.74857300 & 3.34028100 \\
\hline $\mathrm{H}$ & 7.43690600 & 3.48648300 & 4.34687500 \\
\hline $\mathrm{H}$ & 7.56189300 & 4.48160600 & 2.87791400 \\
\hline C & 3.70131400 & 2.54124800 & -1.07337600 \\
\hline $\mathrm{H}$ & 3.34214500 & 1.59965400 & -0.63803100 \\
\hline H & 3.19229500 & 3.35966300 & -0.53170900 \\
\hline C & 3.26139600 & 2.55918200 & -2.54548000 \\
\hline $\mathrm{H}$ & 3.72765600 & 1.68710200 & -3.03877900 \\
\hline C & 3.69430100 & 3.82991900 & -3.28516500 \\
\hline $\mathrm{H}$ & 3.32557700 & 3.82833700 & -4.32368700 \\
\hline $\mathrm{H}$ & 3.29259900 & 4.73148800 & -2.78963200 \\
\hline $\mathrm{H}$ & 4.79084000 & 3.92745600 & -3.32729500 \\
\hline C & 1.74178800 & 2.34971300 & -2.6165730 \\
\hline
\end{tabular}




$\begin{array}{lrrr}\mathrm{H} & 1.20484400 & 3.16101100 & -2.09356500 \\ \mathrm{H} & 1.39045100 & 2.32633700 & -3.66078500 \\ \mathrm{H} & 1.46774800 & 1.39396300 & -2.14215500 \\ \mathrm{H} & 2.35800400 & 2.63905000 & 4.36057400 \\ \mathrm{C} & -3.92077600 & 3.59753200 & 1.29767500 \\ \mathrm{H} & -3.16381900 & 4.39586100 & 1.20392200 \\ \mathrm{H} & -3.56862700 & 2.93594300 & 2.11457700 \\ \mathrm{C} & -5.31650200 & 4.16203900 & 1.61838700 \\ \mathrm{C} & -5.38543100 & 4.62582400 & 3.08308700 \\ \mathrm{H} & -6.38738900 & 5.01440800 & 3.33133700 \\ \mathrm{H} & -4.65346600 & 5.42993800 & 3.26981500 \\ \mathrm{H} & -5.16002000 & 3.79858400 & 3.77462100 \\ \mathrm{C} & -2.84089400 & 3.07366100 & -2.49309200 \\ \mathrm{H} & -3.53707500 & 2.43314700 & -3.04266000 \\ \mathrm{H} & -1.79678300 & 2.75292400 & -2.44199200 \\ \mathrm{C} & -3.23274900 & 4.27178400 & -2.00468600 \\ \mathrm{H} & -4.27925900 & 4.56897400 & -2.14017000 \\ \mathrm{C} & -2.31649200 & 5.28078500 & -1.38308400 \\ \mathrm{H} & -2.79479900 & 5.79188400 & -0.53564100 \\ \mathrm{H} & -1.37447400 & 4.82365900 & -1.04645100 \\ \mathrm{H} & -2.08065100 & 6.05723700 & -2.13247900 \\ \mathrm{H} & -6.05986200 & 3.35089000 & 1.50030700 \\ \mathrm{C} & -5.71227600 & 5.29754400 & 0.66816100 \\ \mathrm{H} & -5.74831000 & 4.95483000 & -0.37616200 \\ \mathrm{H} & -6.70829200 & 5.69613400 & 0.91778600 \\ \mathrm{H} & -4.99233400 & 6.13150600 & 0.73743900\end{array}$

$\mathrm{MgCl}_{2}$-Ti-i sobutyl-DiBS-Mg2-C-Propylene-1, 2-si-TS

$\begin{array}{lrrr}\text { Cl } & 6.09401700 & -3.06661200 & -1.60489300 \\ \text { Mg } & 4.90036000 & -4.63543400 & -0.05813200 \\ \text { Cl } & 7.45696300 & -1.16595400 & 0.92078100 \\ \text { Mg } & 5.31483600 & -1.03277100 & -0.36944200 \\ \text { Cl } & 2.75672400 & -4.50010000 & -1.34842100 \\ \text { Cl } & -0.16597600 & -2.32943600 & -1.40272500 \\ \text { Cl } & 3.17232500 & -0.89588300 & -1.65848000 \\ \text { Cl } & 0.78394400 & -4.03364200 & 1.43406200 \\ \text { Mg } & -1.35955000 & -3.89831200 & 0.14386500 \\ \text { Cl } & 4.12104900 & -2.60017100 & 1.17763400 \\ \text { Mg } & 1.97755000 & -2.46492200 & -0.11270200 \\ \text { Cl } & 1.19837500 & -0.42969700 & 1.12321700 \\ \text { Mg } & -0.94557500 & -0.29444700 & -0.16719800 \\ \text { Cl } & -3.50299000 & -3.76294700 & -1.14635100 \\ \text { Cl } & -6.42584200 & -1.59239900 & -1.20088700 \\ \text { Cl } & -3.08489500 & -0.12400600 & -1.52930200\end{array}$




\begin{tabular}{|c|c|c|c|}
\hline $\mathrm{Cl}$ & -5.47607900 & -3.29650400 & 1.63599700 \\
\hline Mg & -7.61947800 & -3.16120000 & 0.34585600 \\
\hline $\mathrm{Cl}$ & -2.13885700 & -1.86306000 & 1.37968500 \\
\hline Mg & -4.28226400 & -1.72780000 & 0.08959200 \\
\hline $\mathrm{Cl}$ & -4.96423800 & 0.38154600 & 1.34691206 \\
\hline Mg & -7.20487700 & 0.44270300 & 0.03494706 \\
\hline $\mathrm{Cl}$ & -9.34865500 & 0.57808300 & -1.2552520 \\
\hline $\mathrm{Cl}$ & -8.39876800 & -1.12596400 & 1.58161100 \\
\hline $\mathrm{Ti}$ & -3.76831100 & 2.13502900 & -0.28572300 \\
\hline $\mathrm{Cl}$ & -6.07987200 & 2.30892800 & -1.10921506 \\
\hline $\mathrm{Cl}$ & -1.53000400 & 1.89165800 & 0.71658806 \\
\hline C & 5.48168500 & 2.59545500 & 0.82867200 \\
\hline $\mathrm{H}$ & 5.14576700 & 3.52596500 & 1.30768600 \\
\hline C & 5.11607900 & 2.71696400 & -0.68405300 \\
\hline $\mathrm{H}$ & 5.42173900 & 3.72875800 & -0.98431300 \\
\hline C & 4.73270000 & 1.46829600 & 1.50972300 \\
\hline C & 5.96912400 & 1.77914100 & -1.52270400 \\
\hline 0 & 6.86502800 & 2.41575900 & -2.25710000 \\
\hline 0 & 4.21181700 & 1.82804500 & 2.66863100 \\
\hline C & 7.83283900 & 1.60684300 & -2.98707200 \\
\hline $\mathrm{H}$ & 7.29397500 & 0.79464600 & -3.49682700 \\
\hline $\mathrm{H}$ & 8.23668300 & 2.30096900 & -3.73656200 \\
\hline C & 8.91436700 & 1.06604300 & -2.06690100 \\
\hline $\mathrm{H}$ & 9.41462100 & 1.88730800 & -1.53063200 \\
\hline $\mathrm{H}$ & 8.51144800 & 0.35245900 & -1.33080500 \\
\hline $\mathrm{H}$ & 9.67005000 & 0.54089500 & -2.67260500 \\
\hline C & 3.52413800 & 0.84542500 & 3.49247600 \\
\hline $\mathrm{H}$ & 3.36288200 & -0.06705000 & 2.90541300 \\
\hline $\mathrm{H}$ & 4.20790700 & 0.61944300 & 4.32576300 \\
\hline C & 2.22165100 & 1.45245000 & 3.96696000 \\
\hline $\mathrm{H}$ & 1.56273700 & 1.65706800 & 3.11071000 \\
\hline $\mathrm{H}$ & 1.70359300 & 0.73921300 & 4.62666400 \\
\hline 0 & 4.62930200 & 0.34070100 & 1.05807600 \\
\hline 0 & 5.89894500 & 0.55877600 & -1.52757400 \\
\hline C & 6.99999300 & 2.41418800 & 1.06240400 \\
\hline $\mathrm{H}$ & 7.51456000 & 3.28172900 & 0.61206500 \\
\hline $\mathrm{H}$ & 7.33089000 & 1.51339800 & 0.52477000 \\
\hline C & 7.45046800 & 2.24331100 & 2.52287500 \\
\hline $\mathrm{H}$ & 6.94173100 & 1.34655900 & 2.92014400 \\
\hline C & 8.95275100 & 1.93873300 & 2.54939400 \\
\hline $\mathrm{H}$ & 9.30897200 & 1.79254800 & 3.58207200 \\
\hline $\mathrm{H}$ & 9.16230100 & 1.01910000 & 1.98164900 \\
\hline $\mathrm{H}$ & 9.53299500 & 2.76951500 & 2.1097860 \\
\hline C & 7.09402600 & 3.44503600 & 3.4044660 \\
\hline
\end{tabular}




\begin{tabular}{|c|c|c|c|}
\hline $\mathrm{H}$ & 6.00586200 & 3.59433200 & 3.47579100 \\
\hline $\mathrm{H}$ & 7.47405000 & 3.30561900 & 4.42955900 \\
\hline $\mathrm{H}$ & 7.54525700 & 4.37173700 & 3.00756700 \\
\hline C & 3.59977100 & 2.54774100 & -0.91769600 \\
\hline $\mathrm{H}$ & 3.27214400 & 1.58362000 & -0.50812200 \\
\hline $\mathrm{H}$ & 3.09747000 & 3.33412900 & -0.32456300 \\
\hline C & 3.10301900 & 2.61608100 & -2.37002600 \\
\hline $\mathrm{H}$ & 3.56220100 & 1.77153300 & -2.91469200 \\
\hline C & 3.48674000 & 3.92148800 & -3.07531600 \\
\hline $\mathrm{H}$ & 3.07852600 & 3.95384000 & -4.09843400 \\
\hline $\mathrm{H}$ & 3.09125100 & 4.79674900 & -2.53000800 \\
\hline $\mathrm{H}$ & 4.57927300 & 4.03824200 & -3.15505000 \\
\hline C & 1.58459000 & 2.38522100 & -2.38968400 \\
\hline $\mathrm{H}$ & 1.05639300 & 3.17448500 & -1.82574300 \\
\hline $\mathrm{H}$ & 1.19304200 & 2.38389700 & -3.41966000 \\
\hline $\mathrm{H}$ & 1.34370800 & 1.41222400 & -1.93129400 \\
\hline $\mathrm{H}$ & 2.39616900 & 2.38547800 & 4.52507300 \\
\hline C & -3.94336700 & 4.07884500 & 0.64607400 \\
\hline $\mathrm{H}$ & -3.05177800 & 4.45380000 & 1.17010900 \\
\hline $\mathrm{H}$ & -4.27378600 & 3.18386100 & 1.26343400 \\
\hline C & -5.09606200 & 5.08778700 & 0.64352900 \\
\hline C & -5.98205100 & 4.87766300 & 1.88410000 \\
\hline $\mathrm{H}$ & -6.82085000 & 5.59198000 & 1.89256200 \\
\hline $\mathrm{H}$ & -5.40631000 & 5.01852500 & 2.81455800 \\
\hline $\mathrm{H}$ & -6.40854700 & 3.86178100 & 1.89522200 \\
\hline C & -2.97624700 & 3.03305300 & -2.13915800 \\
\hline $\mathrm{H}$ & -3.77851100 & 2.74403100 & -2.82564700 \\
\hline $\mathrm{H}$ & -1.99984500 & 2.56627400 & -2.30431100 \\
\hline C & -3.03794000 & 4.26551400 & -1.47774600 \\
\hline $\mathrm{H}$ & -3.91875400 & 4.88404300 & -1.66580300 \\
\hline C & -1.77438500 & 4.98248400 & -1.08128500 \\
\hline $\mathrm{H}$ & -1.94413000 & 5.78766400 & -0.35566200 \\
\hline $\mathrm{H}$ & -1.03696800 & 4.27897400 & -0.67030900 \\
\hline $\mathrm{H}$ & -1.34438400 & 5.43454000 & -1.99190100 \\
\hline $\mathrm{H}$ & -5.72980600 & 4.88164900 & -0.23509500 \\
\hline C & -4.60534400 & 6.53867100 & 0.56756300 \\
\hline $\mathrm{H}$ & -4.03667100 & 6.74334100 & -0.35208200 \\
\hline $\mathrm{H}$ & -5.45685900 & 7.23674900 & 0.58599200 \\
\hline $\mathrm{H}$ & -3.95494200 & 6.77982900 & 1.42585800 \\
\hline
\end{tabular}

$\mathrm{MgCl}_{2}$-Ti-isobutyl-DiBS-Mg2-C-Propylene-2,1-re-coordination

$\begin{array}{llll}\text { Cl } & -5.85774100 & -3.29496200 & 1.23360900 \\ \text { Mg } & -4.56812300 & -4.74163000 & -0.35464600 \\ \text { Cl } & -7.22261600 & -1.31891000 & -1.23245200\end{array}$




\begin{tabular}{|c|c|c|c|}
\hline Mg & -5.12099600 & -1.17578700 & 0.12174300 \\
\hline $\mathrm{Cl}$ & -2.46508800 & -4.59630100 & 0.99975300 \\
\hline $\mathrm{Cl}$ & 0.37452500 & -2.33026500 & 1.24184100 \\
\hline $\mathrm{Cl}$ & -3.01910000 & -1.02888500 & 1.47494300 \\
\hline $\mathrm{Cl}$ & -0.43886100 & -3.92233600 & -1.70058500 \\
\hline Mg & 1.66398200 & -3.77703400 & -0.34636600 \\
\hline $\mathrm{Cl}$ & -3.83132000 & -2.62101100 & -1.46676600 \\
\hline Mg & -1.72845600 & -2.47570700 & -0.11224600 \\
\hline $\mathrm{Cl}$ & -0.99170100 & -0.35513300 & -1.22461700 \\
\hline Mg & 1.11200100 & -0.20996400 & 0.12992200 \\
\hline $\mathrm{Cl}$ & 3.76691100 & -3.63165500 & 1.00805900 \\
\hline $\mathrm{Cl}$ & 6.60665500 & -1.36576100 & 1.25037400 \\
\hline $\mathrm{Cl}$ & 3.22458300 & -0.00411200 & 1.50324000 \\
\hline $\mathrm{Cl}$ & 5.79345500 & -2.95769500 & -1.69212400 \\
\hline Mg & 7.89627800 & -2.81237600 & -0.33788400 \\
\hline $\mathrm{Cl}$ & 2.40084800 & -1.65641900 & -1.45846200 \\
\hline Mg & 4.50369400 & -1.51127800 & -0.10420500 \\
\hline $\mathrm{Cl}$ & 5.19690100 & 0.67084400 & -1.28447600 \\
\hline Mg & 7.34294400 & 0.75475100 & 0.13814500 \\
\hline $\mathrm{Cl}$ & 9.44639800 & 0.90011200 & 1.49249400 \\
\hline $\mathrm{Cl}$ & 8.63303500 & -0.69178800 & -1.45001600 \\
\hline $\mathrm{Ti}$ & 3.91123200 & 2.36563300 & 0.19152000 \\
\hline $\mathrm{Cl}$ & 6.00762700 & 2.42784700 & 1.40912700 \\
\hline $\mathrm{Cl}$ & 1.55841400 & 2.06616400 & -0.54065900 \\
\hline $\mathrm{C}$ & -5.66555000 & 2.53578900 & -0.48966800 \\
\hline $\mathrm{H}$ & -5.40643000 & 3.56686100 & -0.76869100 \\
\hline C & -5.49496400 & 2.44843000 & 1.05653900 \\
\hline $\mathrm{H}$ & -5.98122100 & 3.34850300 & 1.45736400 \\
\hline C & -4.70365700 & 1.64286800 & -1.25163900 \\
\hline C & -6.26540100 & 1.27809200 & 1.63933200 \\
\hline 0 & -7.30749800 & 1.64620500 & 2.36230700 \\
\hline 0 & -4.10328600 & 2.28741900 & -2.23571000 \\
\hline C & -8.17490800 & 0.59744900 & 2.88452900 \\
\hline $\mathrm{H}$ & -7.54389900 & -0.21262700 & 3.27830600 \\
\hline $\mathrm{H}$ & -8.70146200 & 1.08696600 & 3.71522000 \\
\hline C & -9.12916200 & 0.09155200 & 1.81744500 \\
\hline $\mathrm{H}$ & -9.72155000 & 0.92173700 & 1.40216600 \\
\hline $\mathrm{H}$ & -8.59484400 & -0.41305900 & 0.99684100 \\
\hline $\mathrm{H}$ & -9.82345900 & -0.63376700 & 2.27106400 \\
\hline C & -3.20676500 & 1.58416700 & -3.14029000 \\
\hline $\mathrm{H}$ & -3.04662400 & 0.56490800 & -2.76915600 \\
\hline $\mathrm{H}$ & -3.73382900 & 1.54090400 & -4.10618200 \\
\hline C & -1.91104200 & 2.36168400 & -3.23231100 \\
\hline $\mathrm{H}$ & -1.40239300 & 2.37925600 & -2.25778900 \\
\hline
\end{tabular}




\begin{tabular}{|c|c|c|c|}
\hline $\mathrm{H}$ & -1.23708900 & 1.86685100 & -3.94835000 \\
\hline 0 & -4.51451700 & 0.45885400 & -1.02062300 \\
\hline 0 & -5.98133300 & 0.10075700 & 1.47770400 \\
\hline C & -7.11346300 & 2.22930300 & -0.93709700 \\
\hline $\mathrm{H}$ & -7.78104900 & 2.93456400 & -0.41062400 \\
\hline $\mathrm{H}$ & -7.37281400 & 1.21158700 & -0.60740100 \\
\hline C & -7.38558100 & 2.28828500 & -2.44921200 \\
\hline $\mathrm{H}$ & -6.72443900 & 1.54577800 & -2.93204800 \\
\hline C & -8.82449700 & 1.83207700 & -2.71755400 \\
\hline $\mathrm{H}$ & -9.04866400 & 1.84142100 & -3.79664500 \\
\hline $\mathrm{H}$ & -8.97339500 & 0.80734300 & -2.34355000 \\
\hline $\mathrm{H}$ & -9.54964300 & 2.49986800 & -2.21943700 \\
\hline C & -7.09937100 & 3.66657200 & -3.05590100 \\
\hline $\mathrm{H}$ & -6.03856000 & 3.94513400 & -2.96168000 \\
\hline $\mathrm{H}$ & -7.34999300 & 3.68249800 & -4.12909700 \\
\hline $\mathrm{H}$ & -7.70613400 & 4.44656400 & -2.56271000 \\
\hline C & -4.01337500 & 2.42821100 & 1.48309100 \\
\hline $\mathrm{H}$ & -3.50154900 & 1.59746700 & 0.97712300 \\
\hline $\mathrm{H}$ & -3.55522900 & 3.36437600 & 1.11501600 \\
\hline C & -3.74501900 & 2.28457500 & 2.99050700 \\
\hline $\mathrm{H}$ & -4.14835800 & 1.30423200 & 3.30279600 \\
\hline C & -4.41962900 & 3.37905400 & 3.82595600 \\
\hline $\mathrm{H}$ & -4.16668700 & 3.27193600 & 4.89316300 \\
\hline $\mathrm{H}$ & -4.08745300 & 4.38197700 & 3.50421000 \\
\hline $\mathrm{H}$ & -5.51741000 & 3.34315000 & 3.74517500 \\
\hline C & -2.23158300 & 2.24201900 & 3.23348200 \\
\hline $\mathrm{H}$ & -1.75674000 & 3.18596500 & 2.91312500 \\
\hline $\mathrm{H}$ & -2.00192600 & 2.09627700 & 4.30143800 \\
\hline $\mathrm{H}$ & -1.77434700 & 1.41275100 & 2.67143500 \\
\hline $\mathrm{H}$ & -2.08872200 & 3.39460600 & -3.56987700 \\
\hline C & 4.07348900 & 3.88421200 & -1.19132100 \\
\hline $\mathrm{H}$ & 4.94173800 & 4.33502400 & -0.65967800 \\
\hline $\mathrm{H}$ & 4.47846200 & 3.37939600 & -2.08858600 \\
\hline C & 3.00092100 & 4.92331700 & -1.55936600 \\
\hline C & 2.34785100 & 4.58115000 & -2.90391500 \\
\hline C & 3.57465200 & 6.34795700 & -1.56530700 \\
\hline $\mathrm{H}$ & 2.19940000 & 4.89447600 & -0.79792600 \\
\hline $\mathrm{H}$ & 1.52717200 & 5.27946700 & -3.13528400 \\
\hline $\mathrm{H}$ & 1.93511300 & 3.56163800 & -2.89774700 \\
\hline $\mathrm{H}$ & 3.08742900 & 4.64647400 & -3.72048900 \\
\hline $\mathrm{H}$ & 2.79826500 & 7.08989900 & -1.81632600 \\
\hline $\mathrm{H}$ & 4.38410200 & 6.44031600 & -2.30900700 \\
\hline $\mathrm{H}$ & 3.99358700 & 6.61737100 & -0.58129700 \\
\hline C & 2.77374600 & 3.13987900 & 2.63639900 \\
\hline
\end{tabular}




$\begin{array}{llll}\text { H } & 3.50860500 & 2.57007400 & 3.22006300 \\ \text { C } & 3.21607500 & 4.19733800 & 1.91897900 \\ \text { H } & 2.52056600 & 4.81667000 & 1.34401500 \\ \text { H } & 4.25380500 & 4.53325500 & 1.98582800 \\ \text { C } & 1.34271100 & 2.73056600 & 2.78584500 \\ \text { H } & 1.22772500 & 1.63948900 & 2.70074100 \\ \text { H } & 0.99779900 & 2.99849500 & 3.79971600 \\ \text { H } & 0.68959400 & 3.21965800 & 2.05144000\end{array}$

$\mathrm{MgCl}_{2}$-Ti-isobutyl-DiBS-Mg2-C-Propylene-2,1-re-TS

$\begin{array}{lrrr}\text { Cl } & 5.86472600 & -3.24400100 & -1.37744300 \\ \text { Mg } & 4.59353000 & -4.75100700 & 0.16900600 \\ \text { Cl } & 7.24770300 & -1.35505500 & 1.14612200 \\ \text { Mg } & 5.13319800 & -1.16868400 & -0.18251100 \\ \text { Cl } & 2.47760000 & -4.56242800 & -1.15973700 \\ \text { Cl } & -0.36966700 & -2.29702900 & -1.29301500 \\ \text { Cl } & 3.01841400 & -0.97857200 & -1.51000800 \\ \text { Cl } & 0.47500800 & -3.99266700 & 1.58191300 \\ \text { Mg } & -1.64077300 & -3.80409700 & 0.25327700 \\ \text { Cl } & 3.86193900 & -2.67425600 & 1.36424400 \\ \text { Mg } & 1.74612600 & -2.48575700 & 0.03545800 \\ \text { Cl } & 1.01466000 & -0.40903200 & 1.23083800 \\ \text { Mg } & -1.10163500 & -0.22054500 & -0.09805100 \\ \text { Cl } & -3.75651600 & -3.61549000 & -1.07540000 \\ \text { Cl } & -6.60402400 & -1.35024000 & -1.20899600 \\ \text { Cl } & -3.21650600 & -0.01447000 & -1.51419500 \\ \text { Cl } & -5.75943700 & -3.04573900 & 1.66608800 \\ \text { Mg } & -7.87515900 & -2.85719700 & 0.33747300 \\ \text { Cl } & -2.37238700 & -1.72736100 & 1.44854200 \\ \text { Mg } & -4.48812500 & -1.53883500 & 0.12000100 \\ \text { Cl } & -5.12205200 & 0.60935400 & 1.31744800 \\ \text { Mg } & -7.33527400 & 0.72637100 & -0.01365700 \\ \text { Cl } & -9.45142600 & 0.91505600 & -1.34225700 \\ \text { Cl } & -8.60671300 & -0.78044800 & 1.53274300 \\ \text { Ti } & -3.84155800 & 2.27739600 & -0.36810900 \\ \text { Cl } & -6.11776100 & 2.53109800 & -1.19499900 \\ \text { Cl } & -1.63833200 & 1.98777800 & 0.73388500 \\ \text { C } & 5.65455000 & 2.51318300 & 0.60761900 \\ \text { H } & 5.39333300 & 3.52574400 & 0.94595700 \\ \text { C } & 5.44746400 & 2.50273300 & -0.93707300 \\ \text { H } & 5.91265800 & 3.42836600 & -1.30302600 \\ \text { C } & 4.71795300 & 1.57171900 & 1.34177900 \\ \text { C } & 6.22007200 & 1.37410100 & -1.59447900 \\ \text { O } & 7.24317100 & 1.79262300 & -2.31729700\end{array}$




\begin{tabular}{|c|c|c|c|}
\hline 0 & 4.13747900 & 2.15322600 & 2.37607000 \\
\hline C & 8.11615100 & 0.78252100 & -2.90289600 \\
\hline$H$ & 7.48948700 & -0.01349600 & -3.33082500 \\
\hline 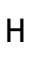 & 8.62688400 & 1.32062500 & -3.71312800 \\
\hline$C$ & 9.08945400 & 0.23326700 & -1.87511000 \\
\hline H & 9.67389300 & 1.04829700 & -1.42047000 \\
\hline - & 8.57262300 & -0.32449300 & -1.07806900 \\
\hline H & 9.78954900 & -0.45467400 & -2.37559100 \\
\hline$C$ & 3.26570500 & 1.38921100 & 3.25495900 \\
\hline$H$ & 3.09890500 & 0.39542600 & 2.82261200 \\
\hline H & 3.81592800 & 1.28504500 & 4.20314900 \\
\hline C & 1.97071000 & 2.15372100 & 3.42740800 \\
\hline H & 1.44056700 & 2.23225200 & 2.46722900 \\
\hline$H$ & 1.31488300 & 1.61035800 & 4.12489000 \\
\hline 0 & 4.52910300 & 0.40219700 & 1.04713600 \\
\hline 0 & 5.95715300 & 0.18573200 & -1.48567300 \\
\hline$C$ & 7.11564500 & 2.19881200 & 1.00520000 \\
\hline $\mathrm{H}$ & 7.76380000 & 2.93783000 & 0.50122000 \\
\hline $\mathrm{H}$ & 7.37716900 & 1.20293800 & 0.61640900 \\
\hline C & 7.42202100 & 2.18231400 & 2.51205300 \\
\hline $\mathrm{H}$ & 6.77844900 & 1.40951200 & 2.97042500 \\
\hline C & 8.87049400 & 1.72691600 & 2.72467600 \\
\hline $\mathrm{H}$ & 9.11870800 & 1.68331700 & 3.79764100 \\
\hline $\mathrm{H}$ & 9.01947500 & 0.72391700 & 2.29606500 \\
\hline $\mathrm{H}$ & 9.57870400 & 2.42618700 & 2.24571400 \\
\hline C & 7.13865900 & 3.52478400 & 3.19505000 \\
\hline $\mathrm{H}$ & 6.07378500 & 3.79803300 & 3.13908000 \\
\hline $\mathrm{H}$ & 7.41334500 & 3.48775800 & 4.26175900 \\
\hline $\mathrm{H}$ & 7.72796900 & 4.33447900 & 2.72936200 \\
\hline C & 3.95627500 & 2.48439700 & -1.32801800 \\
\hline $\mathrm{H}$ & 3.46866200 & 1.61966000 & -0.85697300 \\
\hline $\mathrm{H}$ & 3.49518400 & 3.39206300 & -0.89735600 \\
\hline C & 3.65005300 & 2.42008800 & -2.83338100 \\
\hline $\mathrm{H}$ & 4.05794300 & 1.46443900 & -3.20993900 \\
\hline C & 4.28731300 & 3.56869600 & -3.62419800 \\
\hline $\mathrm{H}$ & 4.00743800 & 3.51661700 & -4.68876800 \\
\hline $\mathrm{H}$ & 3.95032200 & 4.54742900 & -3.23908600 \\
\hline $\mathrm{H}$ & 5.38734700 & 3.54489700 & -3.57387800 \\
\hline C & 2.13117700 & 2.36711500 & -3.03926500 \\
\hline $\mathrm{H}$ & 1.64947700 & 3.28455700 & -2.65785000 \\
\hline $\mathrm{H}$ & 1.87506300 & 2.27354800 & -4.10693500 \\
\hline $\mathrm{H}$ & 1.70136800 & 1.50201500 & -2.51054500 \\
\hline $\mathrm{H}$ & 2.15398200 & 3.16316000 & 3.82746100 \\
\hline C & -3.95684500 & 4.23898300 & 0.52335100 \\
\hline
\end{tabular}




$\begin{array}{llll}\text { H } & -4.85335800 & 4.83368300 & 0.29636200 \\ \text { H } & -4.34895300 & 3.39454100 & 1.17786400 \\ \text { C } & -2.90272300 & 5.03466700 & 1.30044200 \\ \text { C } & -2.97037100 & 4.71170000 & 2.80206000 \\ \text { C } & -3.04747500 & 6.54174700 & 1.05367600 \\ \text { H } & -1.90628500 & 4.71595500 & 0.95018600 \\ \text { H } & -2.20789100 & 5.28003900 & 3.35868000 \\ \text { H } & -2.79030500 & 3.64037800 & 2.98078400 \\ \text { H } & -3.95770300 & 4.97348900 & 3.21876000 \\ \text { H } & -2.24908800 & 7.10169400 & 1.56585000 \\ \text { H } & -4.01513600 & 6.90862000 & 1.43599200 \\ \text { H } & -2.99787700 & 6.79542000 & -0.01731400 \\ \text { C } & -2.93818100 & 3.11855500 & -2.27065000 \\ \text { H } & -3.67668600 & 2.64545500 & -2.93318200 \\ \text { C } & -3.32368000 & 4.31488300 & -1.66856600 \\ \text { H } & -2.55449100 & 5.00816500 & -1.32312300 \\ \text { H } & -4.29693100 & 4.75002000 & -1.89994000 \\ \text { C } & -1.48580100 & 2.77827600 & -2.53203000 \\ \text { H } & -1.32039100 & 1.69283400 & -2.59684400 \\ \text { H } & -1.16840500 & 3.20291800 & -3.49995900 \\ \text { H } & -0.82761600 & 3.18027200 & -1.74988200\end{array}$

$\begin{array}{lrrr}\mathrm{MgCl}_{2} \text {-Ti-isobutyl-DiBS-Mg2-C-Propylene-2,1-si-coordination } \\ \mathrm{Cl} & 5.93728400 & 3.27793500 & 1.39192700 \\ \mathrm{Mg} & 4.67723700 & 4.68769700 & -0.25226400 \\ \mathrm{Cl} & 7.35050900 & 1.24947000 & -1.00332700 \\ \mathrm{Mg} & 5.22347500 & 1.13387900 & 0.31329600 \\ \mathrm{Cl} & 2.54878500 & 4.56990500 & 1.06458500 \\ \mathrm{Cl} & -0.29336900 & 2.30659900 & 1.30227800 \\ \mathrm{Cl} & 3.09618400 & 1.01449000 & 1.62900200 \\ \mathrm{Cl} & 0.57506800 & 3.83415000 & -1.65842100 \\ \mathrm{Mg} & -1.55322600 & 3.71637800 & -0.34171700 \\ \mathrm{Cl} & 3.96337300 & 2.54219500 & -1.33111700 \\ \mathrm{Mg} & 1.83498700 & 2.42453800 & -0.01430200 \\ \mathrm{Cl} & 1.12138000 & 0.27889800 & -1.09310400 \\ \mathrm{Mg} & -1.00827100 & 0.16074200 & 0.22362200 \\ \mathrm{Cl} & -3.68162700 & 3.59853700 & 0.97519500 \\ \mathrm{Cl} & -6.52386000 & 1.33514600 & 1.21302000 \\ \mathrm{Cl} & -3.17371400 & -0.01412200 & 1.56045500 \\ \mathrm{Cl} & -5.65557700 & 2.86273900 & -1.74776900 \\ \mathrm{Mg} & -7.78379700 & 2.74501900 & -0.43103200 \\ \mathrm{Cl} & -2.26710900 & 1.57080500 & -1.42061100 \\ \mathrm{Mg} & -4.39494100 & 1.45417500 & -0.10350300 \\ \mathrm{Cl} & -5.03901500 & -0.71930100 & -1.25098000 \\ & & & \\ & & & 5\end{array}$




\begin{tabular}{|c|c|c|c|}
\hline Mg & -7.23672900 & -0.81026800 & 0.13414300 \\
\hline $\mathrm{Cl}$ & -9.36598000 & -0.92789700 & 1.45098200 \\
\hline $\mathrm{Cl}$ & -8.49768100 & 0.59951300 & -1.50987500 \\
\hline $\mathrm{Ti}$ & -3.80313500 & -2.41599500 & 0.25258900 \\
\hline $\mathrm{Cl}$ & -5.99058400 & -2.61827800 & 1.27498700 \\
\hline $\mathrm{Cl}$ & -1.32870600 & -2.19725400 & -0.14692800 \\
\hline C & 5.52908100 & -2.56766400 & -0.58674600 \\
\hline $\mathrm{H}$ & 5.22172100 & -3.54770300 & -0.97784000 \\
\hline C & 5.20925800 & -2.58033400 & 0.94033900 \\
\hline $\mathrm{H}$ & 5.56928800 & -3.55009100 & 1.31074700 \\
\hline C & 4.71842500 & -1.53321900 & -1.34038800 \\
\hline C & 6.03436900 & -1.53927700 & 1.67782800 \\
\hline 0 & 6.97599200 & -2.06846000 & 2.43964000 \\
\hline 0 & 4.19139900 & -2.01237000 & -2.45259200 \\
\hline C & 7.91179000 & -1.15559800 & 3.08352000 \\
\hline $\mathrm{H}$ & 7.33988900 & -0.32955900 & 3.53104600 \\
\hline $\mathrm{H}$ & 8.35768600 & -1.76365500 & 3.88235500 \\
\hline C & 8.95448800 & -0.64462500 & 2.10383400 \\
\hline $\mathrm{H}$ & 9.48489200 & -1.48431900 & 1.62851100 \\
\hline $\mathrm{H}$ & 8.50873000 & -0.01356900 & 1.31875500 \\
\hline $\mathrm{H}$ & 9.69313400 & -0.03742700 & 2.65110000 \\
\hline C & 3.45572300 & -1.13277000 & -3.34761200 \\
\hline $\mathrm{H}$ & 3.26452500 & -0.17966000 & -2.83944000 \\
\hline $\mathrm{H}$ & 4.12045000 & -0.95351500 & -4.20729900 \\
\hline C & 2.17364600 & -1.82951100 & -3.74965300 \\
\hline $\mathrm{H}$ & 1.53279700 & -1.98610300 & -2.86994800 \\
\hline $\mathrm{H}$ & 1.62004000 & -1.19698500 & -4.46085800 \\
\hline 0 & 4.57864400 & -0.37489100 & -0.98592800 \\
\hline 0 & 5.89908100 & -0.32768000 & 1.58733900 \\
\hline C & 7.03258900 & -2.34417700 & -0.87505200 \\
\hline $\mathrm{H}$ & 7.59417900 & -3.14289900 & -0.35848600 \\
\hline $\mathrm{H}$ & 7.33283000 & -1.38357400 & -0.43061200 \\
\hline C & 7.44563600 & -2.29424000 & -2.35572000 \\
\hline $\mathrm{H}$ & 6.89077800 & -1.46175600 & -2.82478200 \\
\hline C & 8.93257400 & -1.93227500 & -2.44785500 \\
\hline $\mathrm{H}$ & 9.26107500 & -1.87230100 & -3.49817500 \\
\hline $\mathrm{H}$ & 9.11412700 & -0.95446700 & -1.97573500 \\
\hline $\mathrm{H}$ & 9.55714200 & -2.69184000 & -1.94483500 \\
\hline C & 7.12308700 & -3.58809800 & -3.11082700 \\
\hline $\mathrm{H}$ & 6.04099000 & -3.78707900 & -3.14132600 \\
\hline $\mathrm{H}$ & 7.47638100 & -3.53214100 & -4.15328000 \\
\hline $\mathrm{H}$ & 7.62079800 & -4.45357000 & -2.63860600 \\
\hline C & 3.69516500 & -2.45542800 & 1.21007600 \\
\hline $\mathrm{H}$ & 3.31592300 & -1.53771200 & 0.7419800 \\
\hline
\end{tabular}




$\begin{array}{llll}\text { H } & 3.20674000 & -3.30349800 & 0.69594500 \\ \mathrm{C} & 3.25191200 & -2.43365300 & 2.68112400 \\ \mathrm{H} & 3.70219400 & -1.53860300 & 3.14746700 \\ \mathrm{C} & 3.70504400 & -3.67239900 & 3.46191000 \\ \mathrm{H} & 3.33334100 & -3.64422300 & 4.49900800 \\ \mathrm{H} & 3.32061700 & -4.59627500 & 2.99473200 \\ \mathrm{H} & 4.80300900 & -3.74911100 & 3.51007700 \\ \mathrm{C} & 1.72872200 & -2.24844000 & 2.74214800 \\ \mathrm{H} & 1.20619700 & -3.08329600 & 2.24297800 \\ \mathrm{H} & 1.37496200 & -2.19917700 & 3.78483900 \\ \mathrm{H} & 1.44044500 & -1.31205500 & 2.23851700 \\ \mathrm{H} & 2.38007600 & -2.79958700 & -4.22798200 \\ \mathrm{C} & -3.90918900 & -3.85135100 & -1.22839500 \\ \mathrm{H} & -3.82730600 & -4.75923900 & -0.59279200 \\ \mathrm{H} & -4.97853000 & -3.75834600 & -1.50967900 \\ \mathrm{C} & -3.01068200 & -3.95856600 & -2.46540000 \\ \mathrm{C} & -3.05295800 & -2.68955000 & -3.32465800 \\ \mathrm{C} & -3.37923900 & -5.19724500 & -3.29812200 \\ \mathrm{H} & -1.97438700 & -4.08670900 & -2.10766700 \\ \mathrm{H} & -2.40898500 & -2.79641800 & -4.21225200 \\ \mathrm{H} & -2.70716100 & -1.81030300 & -2.76128800 \\ \mathrm{H} & -4.07857600 & -2.48124800 & -3.67255500 \\ \mathrm{H} & -2.70215600 & -5.30971200 & -4.16133900 \\ \mathrm{H} & -4.40883000 & -5.11476100 & -3.68627300 \\ \mathrm{H} & -3.32029500 & -6.11997700 & -2.69818100 \\ \mathrm{C} & -2.85233200 & -3.08447300 & 2.88497100 \\ \mathrm{C} & -3.08234600 & -4.14480900 & 2.08055100 \\ \mathrm{H} & -2.30913100 & -4.49276100 & 1.38831500 \\ \mathrm{H} & -3.99173600 & -4.74590400 & 2.17844700 \\ \mathrm{H} & -1.90271500 & -2.54876600 & 2.76479400 \\ \mathrm{H} & -3.76489400 & -2.59390400 & 3.96101200 \\ \mathrm{H} & -3.26723900 & -2.71765300 & 4.93892900 \\ & -3.96271000 & -1.51762900 & 3.84043100 \\ -4.72233500 & -3.13199900 & 3.97236300\end{array}$

$\mathrm{MgCl}_{2}$-Ti-i sobutyl-DiBS-Mg2-C-Propylene-2,1-si-TS

$\begin{array}{lrrr}\text { Cl } & 5.99122700 & -3.19816500 & -1.63271900 \\ \text { Mg } & 4.78576600 & -4.70267400 & -0.03214800 \\ \text { Cl } & 7.42725300 & -1.26521100 & 0.82708600 \\ \text { Mg } & 5.27133100 & -1.11840400 & -0.43844900 \\ \text { Cl } & 2.62839100 & -4.55367700 & -1.29777400 \\ \text { Cl } & -0.24868400 & -2.32339000 & -1.36864600 \\ \text { Cl } & 3.11511800 & -0.96784700 & -1.70288100 \\ \text { Cl } & 0.70315700 & -3.97697300 & 1.49731800\end{array}$




\begin{tabular}{|c|c|c|c|}
\hline Mg & -1.45405100 & -3.82797800 & 0.23179600 \\
\hline $\mathrm{Cl}$ & 4.06579900 & -2.62147900 & 1.16241500 \\
\hline Mg & 1.90856300 & -2.47253400 & -0.10329100 \\
\hline $\mathrm{Cl}$ & 1.18871800 & -0.39139500 & 1.09141700 \\
\hline Mg & -0.96979300 & -0.24244900 & -0.17416000 \\
\hline $\mathrm{Cl}$ & -3.61129600 & -3.67896100 & -1.03378100 \\
\hline $\mathrm{Cl}$ & -6.48864100 & -1.44885900 & -1.10484500 \\
\hline $\mathrm{Cl}$ & -3.12307700 & -0.06631200 & -1.51437800 \\
\hline $\mathrm{Cl}$ & -5.53681600 & -3.10227300 & 1.76116100 \\
\hline Mg & -7.69399400 & -2.95329900 & 0.49565800 \\
\hline $\mathrm{Cl}$ & -2.17397700 & -1.74691000 & 1.42634500 \\
\hline Mg & -4.33123000 & -1.59753900 & 0.16103500 \\
\hline $\mathrm{Cl}$ & -4.94067900 & 0.55514400 & 1.36487800 \\
\hline Mg & -7.20742100 & 0.63191300 & 0.08957100 \\
\hline $\mathrm{Cl}$ & -9.36580000 & 0.78133600 & -1.17574700 \\
\hline $\mathrm{Cl}$ & -8.41399000 & -0.87204200 & 1.69025800 \\
\hline $\mathrm{Ti}$ & -3.75168900 & 2.25362700 & -0.33492900 \\
\hline $\mathrm{Cl}$ & -6.08048600 & 2.48039400 & -1.07760400 \\
\hline $\mathrm{Cl}$ & -1.50679300 & 1.97685500 & 0.65347100 \\
\hline C & 5.38614900 & 2.51809800 & 0.78268800 \\
\hline $\mathrm{H}$ & 5.05497200 & 3.43850100 & 1.28385200 \\
\hline C & 4.93955600 & 2.63574400 & -0.71041400 \\
\hline $\mathrm{H}$ & 5.19443600 & 3.65834100 & -1.02089300 \\
\hline C & 4.69781200 & 1.36718000 & 1.48407100 \\
\hline C & 5.78847000 & 1.73230700 & -1.59209000 \\
\hline 0 & 6.61388200 & 2.40868600 & -2.37342600 \\
\hline 0 & 4.21184500 & 1.69489400 & 2.66728000 \\
\hline C & 7.58406200 & 1.64380700 & -3.14525700 \\
\hline $\mathrm{H}$ & 7.06053100 & 0.80904500 & -3.63422700 \\
\hline $\mathrm{H}$ & 7.92527600 & 2.35523000 & -3.90945800 \\
\hline C & 8.72795400 & 1.15044800 & -2.27461300 \\
\hline $\mathrm{H}$ & 9.21055200 & 1.99169000 & -1.75346800 \\
\hline $\mathrm{H}$ & 8.39046500 & 0.41437100 & -1.52831600 \\
\hline $\mathrm{H}$ & 9.48193400 & 0.66514100 & -2.91461500 \\
\hline C & 3.60019600 & 0.67082300 & 3.50088600 \\
\hline $\mathrm{H}$ & 3.39791800 & -0.21694000 & 2.88899300 \\
\hline $\mathrm{H}$ & 4.35057400 & 0.41245100 & 4.26475900 \\
\hline C & 2.33746700 & 1.24454800 & 4.10527700 \\
\hline $\mathrm{H}$ & 1.60964800 & 1.47778900 & 3.31463700 \\
\hline $\mathrm{H}$ & 1.88094800 & 0.50003400 & 4.77594500 \\
\hline 0 & 4.61417200 & 0.24310100 & 1.02190900 \\
\hline 0 & 5.78670500 & 0.50983500 & -1.58666200 \\
\hline C & 6.91622100 & 2.36029800 & 0.94460300 \\
\hline $\mathrm{H}$ & 7.39758500 & 3.23494300 & 0.4719250 \\
\hline
\end{tabular}




\begin{tabular}{|c|c|c|c|}
\hline $\mathrm{H}$ & 7.23516400 & 1.46373000 & 0.39285200 \\
\hline C & 7.43440500 & 2.19429500 & 2.38310800 \\
\hline $\mathrm{H}$ & 6.95798200 & 1.28899900 & 2.80069500 \\
\hline C & 8.94055400 & 1.91108300 & 2.34231500 \\
\hline $\mathrm{H}$ & 9.34432700 & 1.77064600 & 3.35814800 \\
\hline $\mathrm{H}$ & 9.13698300 & 0.99336000 & 1.76664300 \\
\hline $\mathrm{H}$ & 9.48934800 & 2.74920300 & 1.87706000 \\
\hline C & 7.09891500 & 3.38893600 & 3.28230400 \\
\hline $\mathrm{H}$ & 6.01272700 & 3.51954300 & 3.40460700 \\
\hline $\mathrm{H}$ & 7.52858800 & 3.25521000 & 4.28837700 \\
\hline $\mathrm{H}$ & 7.51501100 & 4.32364400 & 2.86624800 \\
\hline C & 3.41808800 & 2.41805400 & -0.85825800 \\
\hline $\mathrm{H}$ & 3.14915100 & 1.43381500 & -0.45636500 \\
\hline $\mathrm{H}$ & 2.92743500 & 3.16863100 & -0.21151300 \\
\hline C & 2.82475600 & 2.51019000 & -2.27243000 \\
\hline $\mathrm{H}$ & 3.29786300 & 1.71958800 & -2.88212100 \\
\hline C & 3.07808500 & 3.86433200 & -2.94375100 \\
\hline $\mathrm{H}$ & 2.60500400 & 3.90860300 & -3.93805800 \\
\hline $\mathrm{H}$ & 2.66256900 & 4.68971700 & -2.33894600 \\
\hline $\mathrm{H}$ & 4.15360300 & 4.05682400 & -3.08526700 \\
\hline C & 1.32386500 & 2.18224000 & -2.20580400 \\
\hline $\mathrm{H}$ & 0.78812700 & 2.89383700 & -1.55341300 \\
\hline $\mathrm{H}$ & 0.86106400 & 2.21750400 & -3.20541600 \\
\hline H & 1.18226200 & 1.16739000 & -1.79810600 \\
\hline $\mathrm{H}$ & 2.55009800 & 2.15590500 & 4.68555200 \\
\hline C & -3.90647800 & 4.22740400 & 0.51549400 \\
\hline $\mathrm{H}$ & -4.81141800 & 4.78503000 & 0.23089500 \\
\hline $\mathrm{H}$ & -4.28345500 & 3.36824300 & 1.15714300 \\
\hline C & -2.94023500 & 5.06373900 & 1.35475100 \\
\hline C & -3.26270300 & 4.90487700 & 2.85100000 \\
\hline C & -2.96542200 & 6.54206700 & 0.94560100 \\
\hline $\mathrm{H}$ & -1.91994100 & 4.67001600 & 1.20406100 \\
\hline $\mathrm{H}$ & -2.57254900 & 5.50602700 & 3.46473700 \\
\hline $\mathrm{H}$ & -3.16660900 & 3.85389100 & 3.16730800 \\
\hline $\mathrm{H}$ & -4.29025800 & 5.23909000 & 3.07268500 \\
\hline $\mathrm{H}$ & -2.24317900 & 7.12383000 & 1.53974800 \\
\hline $\mathrm{H}$ & -3.96598200 & 6.97503500 & 1.11480100 \\
\hline $\mathrm{H}$ & -2.71462500 & 6.68826500 & -0.11617000 \\
\hline C & -2.88752800 & 3.07828500 & -2.26128600 \\
\hline C & -2.92930200 & 4.26567400 & -1.53273600 \\
\hline $\mathrm{H}$ & -2.02921800 & 4.61199500 & -1.02340900 \\
\hline $\mathrm{H}$ & -3.66121100 & 5.03306500 & -1.79505000 \\
\hline $\mathrm{H}$ & -1.93740200 & 2.53067700 & -2.23036500 \\
\hline C & -3.77870400 & 2.79630900 & -3.4512050 \\
\hline
\end{tabular}




$\begin{array}{llll}H & -3.24100900 & 3.05889200 & -4.37937400 \\ H & -4.05049900 & 1.73337100 & -3.51847700 \\ H & -4.70784100 & 3.38105500 & -3.41021500\end{array}$

\begin{tabular}{|c|c|c|c|}
\hline $\mathrm{Cl}$ & -7.79932200 & -1.24703400 & 0.90086700 \\
\hline Mg & -6.82889300 & -3.05349900 & -0.53886300 \\
\hline $\mathrm{Cl}$ & -8.59198800 & 0.81807600 & -1.73694100 \\
\hline Mg & -6.55697600 & 0.56924800 & -0.29667300 \\
\hline $\mathrm{Cl}$ & -4.79388300 & -3.30232800 & 0.90140500 \\
\hline $\mathrm{Cl}$ & -1.51650800 & -1.73488100 & 1.14414000 \\
\hline $\mathrm{Cl}$ & -4.52196600 & 0.32041800 & 1.14359400 \\
\hline $\mathrm{Cl}$ & -2.58111000 & -3.29250800 & -1.73586700 \\
\hline Mg & -0.54615200 & -3.54132800 & -0.29564000 \\
\hline $\mathrm{Cl}$ & -5.58654900 & -1.23721500 & -1.73640400 \\
\hline Mg & -3.55154200 & -1.48603600 & -0.29612700 \\
\hline $\mathrm{Cl}$ & -2.30920900 & 0.33022700 & -1.49365600 \\
\hline Mg & -0.27383300 & 0.08137700 & -0.05330500 \\
\hline $\mathrm{Cl}$ & 1.48883600 & -3.79011500 & 1.14467100 \\
\hline $\mathrm{Cl}$ & 4.76626400 & -2.22266100 & 1.38749400 \\
\hline $\mathrm{Cl}$ & 1.87350200 & -0.08736500 & 1.31946900 \\
\hline $\mathrm{Cl}$ & 3.70179000 & -3.78034300 & -1.49253700 \\
\hline Mg & 5.73669200 & -4.02918100 & -0.05233100 \\
\hline $\mathrm{Cl}$ & 0.69629700 & -1.72505100 & -1.49315400 \\
\hline Mg & 2.73120500 & -1.97398900 & -0.05305400 \\
\hline $\mathrm{Cl}$ & 4.04656900 & -0.18134200 & -1.41667900 \\
\hline Mg & 6.00827100 & -0.40641800 & 0.18981300 \\
\hline $\mathrm{Cl}$ & 8.04363600 & -0.65526700 & 1.63010700 \\
\hline $\mathrm{Cl}$ & 6.97906400 & -2.21287200 & -1.24988700 \\
\hline $\mathrm{Ti}$ & 3.13014300 & 1.90991700 & -0.28176600 \\
\hline $\mathrm{Cl}$ & 5.07838800 & 1.67413200 & 1.14783900 \\
\hline $\mathrm{Cl}$ & 0.99850100 & 1.78895500 & -1.43029400 \\
\hline C & -3.84554600 & 5.14725900 & -0.64800800 \\
\hline C & -2.70059000 & 4.50949100 & -1.13581100 \\
\hline C & -2.04946600 & 3.55139900 & -0.35853400 \\
\hline C & -2.54199600 & 3.23612300 & 0.91755700 \\
\hline C & -3.68842100 & 3.88471700 & 1.41178500 \\
\hline C & -4.33836400 & 4.83544600 & 0.62702500 \\
\hline $\mathrm{H}$ & -5.23263900 & 5.33631400 & 1.00573900 \\
\hline $\mathrm{H}$ & -4.36007500 & 5.89026400 & -1.26291500 \\
\hline $\mathrm{H}$ & -2.31861800 & 4.74698800 & -2.13104800 \\
\hline $\mathrm{H}$ & -1.16846400 & 3.03749300 & -0.73957900 \\
\hline H & -4.06835900 & 3.62235900 & 2.39946900 \\
\hline
\end{tabular}




$\begin{array}{lrrr}\text { C } & -1.87281800 & 2.18049800 & 1.70310300 \\ \text { O } & -2.31057700 & 2.04926800 & 2.93513900 \\ \text { C } & -1.81654300 & 0.94020600 & 3.72890600 \\ \text { C } & -2.59141400 & 0.93681700 & 5.02647200 \\ \text { H } & -1.96659400 & 0.01569000 & 3.15503600 \\ \text { H } & -0.73285000 & 1.07402500 & 3.87438600 \\ \text { H } & -3.66522100 & 0.79961600 & 4.82855500 \\ \text { H } & -2.45060900 & 1.87951200 & 5.57715300 \\ \text { H } & -2.24570100 & 0.10690800 & 5.66144000 \\ \text { O } & -0.96014200 & 1.46988000 & 1.26582400 \\ \text { C } & 3.81774000 & 3.24659700 & -1.71367500 \\ \text { H } & 4.68186600 & 3.63238700 & -1.13406500 \\ \text { H } & 4.23461500 & 2.57572500 & -2.48860100 \\ \text { C } & 2.97309000 & 4.37278500 & -2.34109200 \\ \text { C } & 2.53738200 & 4.00497000 & -3.76418100 \\ \text { C } & 3.71271900 & 5.71799000 & -2.31495800 \\ \text { H } & 2.04357900 & 4.49489700 & -1.75249400 \\ \text { H } & 1.87211300 & 4.77456400 & -4.18922500 \\ \text { H } & 1.99907900 & 3.04577700 & -3.77453000 \\ \text { H } & 3.41539500 & 3.91333000 & -4.42645300 \\ \text { H } & 3.09493800 & 6.52483300 & -2.74374100 \\ \text { H } & 4.64745400 & 5.66060600 & -2.89784800 \\ \text { H } & 3.98343400 & 6.01162400 & -1.28653000 \\ \text { C } & 1.91170700 & 3.24530500 & 1.58315600 \\ \text { H } & 2.18132500 & 2.65160400 & 2.46283600 \\ \text { H } & 0.93832800 & 3.04566200 & 1.13094500 \\ \text { C } & 2.70369800 & 4.25110600 & 1.14802600 \\ \text { H } & 2.36323000 & 4.83242600 & 0.28295800 \\ \text { C } & 3.96224400 & 4.72968200 & 1.80045200 \\ \text { H } & 4.78909100 & 4.79347500 & 1.07602200 \\ \text { H } & 3.79702900 & 5.75068200 & 2.18730400 \\ \text { H } & 4.27516500 & 4.07943600 & 2.62835700 \\ & & & \\ \text { Hon } & & \end{array}$

$\mathrm{MgCl}_{2}$-Ti-isobutyl-EB-Mg1-Propylene-1, 2-re-insertion-TS

$\begin{array}{lrrr}\text { Cl } & -7.80170900 & -1.22471000 & 0.91030800 \\ \text { Mg } & -6.84138400 & -3.04509500 & -0.51862400 \\ \text { Cl } & -8.59244600 & 0.82452700 & -1.74042700 \\ \text { Mg } & -6.55516500 & 0.57822400 & -0.30293500 \\ \text { Cl } & -4.80410600 & -3.29139700 & 0.91886800 \\ \text { Cl } & -1.52029600 & -1.73475600 & 1.14311300 \\ \text { Cl } & -4.51788600 & 0.33192100 & 1.13455700 \\ \text { Cl } & -2.59723800 & -3.30884400 & -1.72330900 \\ \text { Mg } & -0.56004900 & -3.55513500 & -0.28589100 \\ \text { Cl } & -5.59484200 & -1.24215800 & -1.73186800\end{array}$




\begin{tabular}{|c|c|c|c|}
\hline Mg & -3.55757700 & -1.48843200 & -0.29434800 \\
\hline $\mathrm{Cl}$ & -2.31102900 & 0.31448900 & -1.50760300 \\
\hline Mg & -0.27306100 & 0.06799200 & -0.06997000 \\
\hline $\mathrm{Cl}$ & 1.47724300 & -3.80139000 & 1.15168600 \\
\hline $\mathrm{Cl}$ & 4.76114500 & -2.24474600 & 1.37604500 \\
\hline $\mathrm{Cl}$ & 1.88330700 & -0.10230000 & 1.33198600 \\
\hline $\mathrm{Cl}$ & 3.68430300 & -3.81889000 & -1.49043100 \\
\hline Mg & 5.72146500 & -4.06520800 & -0.05299800 \\
\hline $\mathrm{Cl}$ & 0.68663600 & -1.75220000 & -1.49908300 \\
\hline Mg & 2.72375400 & -1.99864600 & -0.06176200 \\
\hline $\mathrm{Cl}$ & 3.99405100 & -0.22109700 & -1.40850800 \\
\hline Mg & 6.00711700 & -0.44176900 & 0.16257800 \\
\hline $\mathrm{Cl}$ & 8.04497900 & -0.68819800 & 1.60015500 \\
\hline $\mathrm{Cl}$ & 6.96805400 & -2.26225000 & -1.26623900 \\
\hline $\mathrm{Ti}$ & 3.01902500 & 1.85757800 & -0.10998700 \\
\hline $\mathrm{Cl}$ & 5.21963200 & 1.72104100 & 1.00143200 \\
\hline $\mathrm{Cl}$ & 1.01638600 & 1.74325700 & -1.46994000 \\
\hline C & -3.78808300 & 5.14172800 & -0.70256900 \\
\hline C & -2.63804100 & 4.50096100 & -1.17454900 \\
\hline C & -1.99742900 & 3.54483300 & -0.38634400 \\
\hline C & -2.50588700 & 3.23454500 & 0.88455400 \\
\hline C & -3.65695100 & 3.88614900 & 1.36325800 \\
\hline C & -4.29637300 & 4.83502300 & 0.56751900 \\
\hline $\mathrm{H}$ & -5.19457100 & 5.33816700 & 0.93372500 \\
\hline $\mathrm{H}$ & -4.29457900 & 5.88297900 & -1.32620300 \\
\hline $\mathrm{H}$ & -2.24465000 & 4.73455500 & -2.16630700 \\
\hline $\mathrm{H}$ & -1.11160400 & 3.02887100 & -0.75343600 \\
\hline $\mathrm{H}$ & -4.04877200 & 3.62719800 & 2.34719400 \\
\hline C & -1.84669500 & 2.18086100 & 1.68080000 \\
\hline 0 & -2.28875400 & 2.06166500 & 2.91176000 \\
\hline C & -1.79928400 & 0.95887700 & 3.71806100 \\
\hline C & -2.58797400 & 0.96133300 & 5.00722900 \\
\hline $\mathrm{H}$ & -1.94007500 & 0.03021400 & 3.14860400 \\
\hline $\mathrm{H}$ & -0.71779600 & 1.09865200 & 3.87378100 \\
\hline $\mathrm{H}$ & -3.65907900 & 0.81877400 & 4.79871100 \\
\hline $\mathrm{H}$ & -2.45625700 & 1.90806900 & 5.55315600 \\
\hline $\mathrm{H}$ & -2.24589900 & 0.13690900 & 5.65123800 \\
\hline 0 & -0.93711200 & 1.46066300 & 1.25140000 \\
\hline C & 3.67344600 & 3.64046500 & -1.15683500 \\
\hline $\mathrm{H}$ & 4.64064000 & 4.08317200 & -0.88012900 \\
\hline $\mathrm{H}$ & 3.95601500 & 2.64774300 & -1.62511500 \\
\hline C & 2.91825300 & 4.46744000 & -2.20298900 \\
\hline C & 3.22315800 & 3.94808700 & -3.61823800 \\
\hline C & 3.22814800 & 5.96523900 & -2.08979400 \\
\hline
\end{tabular}




$\begin{array}{llll}\mathrm{H} & 1.83671800 & 4.32134300 & -2.03899500 \\ \mathrm{H} & 2.67768000 & 4.53237300 & -4.37709700 \\ \mathrm{H} & 2.91939100 & 2.89455200 & -3.72149800 \\ \mathrm{H} & 4.30042500 & 4.02241000 & -3.84456100 \\ \mathrm{H} & 2.67101400 & 6.53719800 & -2.84871000 \\ \mathrm{H} & 4.30328700 & 6.15645900 & -2.24769100 \\ \mathrm{H} & 2.95722700 & 6.37576700 & -1.10485800 \\ \mathrm{C} & 2.14468500 & 3.07927200 & 1.49437600 \\ \mathrm{H} & 2.64025100 & 2.71571400 & 2.40107000 \\ \mathrm{H} & 1.09207400 & 2.81198700 & 1.36700100 \\ \mathrm{C} & 2.66126700 & 4.19534000 & 0.82325800 \\ \mathrm{H} & 1.96626700 & 4.74354800 & 0.18215800 \\ \mathrm{C} & 3.79973600 & 4.98851800 & 1.41033600 \\ \mathrm{H} & 4.26172100 & 5.67546900 & 0.68967200 \\ \mathrm{H} & 3.39546100 & 5.59286700 & 2.24089600 \\ \mathrm{H} & 4.57528700 & 4.32533000 & 1.81816200\end{array}$

$\mathrm{MgCl}_{2}$-Ti-isobutyl-EB-Mg1-Propylene-1,2-si-coordination

$\begin{array}{lrrr}\text { Cl } & -7.90987100 & -1.11221000 & 0.76472500 \\ \text { Mg } & -6.92933800 & -2.95400700 & -0.62243200 \\ \text { Cl } & -8.62601400 & 0.91349300 & -1.92501400 \\ \text { Mg } & -6.62384300 & 0.66949700 & -0.43863000 \\ \text { Cl } & -4.92716800 & -3.19800400 & 0.86395200 \\ \text { Cl } & -1.63895700 & -1.66029400 & 1.14699300 \\ \text { Cl } & -4.62167400 & 0.42549900 & 1.04775300 \\ \text { Cl } & -2.66061100 & -3.25809300 & -1.72656500 \\ \text { Mg } & -0.65851300 & -3.50208300 & -0.24024800 \\ \text { Cl } & -5.64331300 & -1.17230000 & -1.82578900 \\ \text { Mg } & -3.64115900 & -1.41626400 & -0.33937600 \\ \text { Cl } & -2.35513000 & 0.36540400 & -1.54274400 \\ \text { Mg } & -0.35239100 & 0.12130700 & -0.05629200 \\ \text { Cl } & 1.34364900 & -3.74603500 & 1.24619500 \\ \text { Cl } & 4.63193000 & -2.20834000 & 1.52933200 \\ \text { Cl } & 1.77171300 & -0.04388200 & 1.34653900 \\ \text { Cl } & 3.61039600 & -3.80616400 & -1.34425100 \\ \text { Mg } & 5.61246100 & -4.05017400 & 0.14207700 \\ \text { Cl } & 0.62763700 & -1.72037100 & -1.44355200 \\ \text { Mg } & 2.62966000 & -1.96452500 & 0.04274400 \\ \text { Cl } & 4.00535900 & -0.25205400 & -1.34349300 \\ \text { Mg } & 5.91745900 & -0.42658200 & 0.32579000 \\ \text { Cl } & 7.92013400 & -0.67068100 & 1.81224300 \\ \text { Cl } & 6.89851600 & -2.26844300 & -1.06129200 \\ \text { Ti } & 3.09279400 & 1.88059100 & -0.33427200 \\ \text { Cl } & 4.97839600 & 1.70885200 & 1.18104400\end{array}$




\begin{tabular}{|c|c|c|c|}
\hline $\mathrm{Cl}$ & 0.92848900 & 1.83323800 & -1.42864600 \\
\hline C & -3.89254100 & 5.22545900 & -0.70050500 \\
\hline C & -2.76406800 & 4.55859600 & -1.18821400 \\
\hline C & -2.12806100 & 3.59559300 & -0.40427400 \\
\hline C & -2.61951000 & 3.30413800 & 0.87787200 \\
\hline C & -3.74978300 & 3.98066700 & 1.37121100 \\
\hline C & -4.38427500 & 4.93686400 & 0.58030700 \\
\hline $\mathrm{H}$ & -5.26608500 & 5.45955400 & 0.95869900 \\
\hline $\mathrm{H}$ & -4.39515100 & 5.97256600 & -1.32028400 \\
\hline $\mathrm{H}$ & -2.38404200 & 4.77694800 & -2.18861000 \\
\hline $\mathrm{H}$ & -1.26103600 & 3.05780800 & -0.78401500 \\
\hline $\mathrm{H}$ & -4.12978700 & 3.73591300 & 2.36336200 \\
\hline C & -1.96493400 & 2.24476200 & 1.67141300 \\
\hline 0 & -2.40871500 & 2.12527600 & 2.90257100 \\
\hline C & -1.91737600 & 1.02426200 & 3.70964600 \\
\hline C & -2.68441700 & 1.04464200 & 5.01164700 \\
\hline $\mathrm{H}$ & -2.07630500 & 0.09274400 & 3.14966200 \\
\hline $\mathrm{H}$ & -0.83202500 & 1.15311700 & 3.84694200 \\
\hline $\mathrm{H}$ & -3.76063600 & 0.91346500 & 4.82280200 \\
\hline $\mathrm{H}$ & -2.53270400 & 1.99344700 & 5.54878500 \\
\hline $\mathrm{H}$ & -2.34113600 & 0.22073400 & 5.65566600 \\
\hline 0 & -1.05696800 & 1.52400900 & 1.24179700 \\
\hline C & 3.80753800 & 2.98442700 & -1.95150800 \\
\hline $\mathrm{H}$ & 3.22033700 & 3.90467000 & -2.12028300 \\
\hline $\mathrm{H}$ & 3.51419800 & 2.28575200 & -2.75939400 \\
\hline C & 5.32015700 & 3.26173700 & -2.00422000 \\
\hline C & 5.77118700 & 3.52705400 & -3.45043200 \\
\hline $\mathrm{H}$ & 6.85859900 & 3.70480800 & -3.50452300 \\
\hline $\mathrm{H}$ & 5.26118900 & 4.41657300 & -3.85843200 \\
\hline $\mathrm{H}$ & 5.53242100 & 2.67413800 & -4.10542400 \\
\hline C & 1.93458000 & 3.27301500 & 1.50637800 \\
\hline $\mathrm{H}$ & 2.43063300 & 2.67697600 & 2.27798600 \\
\hline $\mathrm{H}$ & 0.88561500 & 3.04594100 & 1.30008500 \\
\hline C & 2.56630000 & 4.31066100 & 0.91440500 \\
\hline $\mathrm{H}$ & 3.60405500 & 4.52108600 & 1.19872100 \\
\hline C & 1.91561900 & 5.26938700 & -0.03549500 \\
\hline $\mathrm{H}$ & 2.59836100 & 5.56240600 & -0.84541600 \\
\hline $\mathrm{H}$ & 0.99858400 & 4.84706300 & -0.47148400 \\
\hline $\mathrm{H}$ & 1.65078200 & 6.19218900 & 0.51077300 \\
\hline $\mathrm{H}$ & 5.85648100 & 2.35757400 & -1.65758300 \\
\hline C & 5.72732100 & 4.42070200 & -1.08765300 \\
\hline $\mathrm{H}$ & 5.49948000 & 4.19759100 & -0.03547000 \\
\hline $\mathrm{H}$ & 6.80826000 & 4.62231300 & -1.15474800 \\
\hline $\mathrm{H}$ & 5.19629500 & 5.34604400 & -1.37219200 \\
\hline
\end{tabular}




\begin{tabular}{|c|c|c|c|}
\hline \multicolumn{4}{|c|}{$\mathrm{MgCl}_{2}$-Ti-isobutyl-EB-Mg1-Propylene-1,2-si-insertion-TS } \\
\hline $\mathrm{Cl}$ & -7.87246100 & -1.06267300 & 0.88691700 \\
\hline Mg & -6.93859500 & -2.94955600 & -0.47155100 \\
\hline $\mathrm{Cl}$ & -8.62350900 & 0.90051000 & -1.83941200 \\
\hline Mg & -6.59387300 & 0.67412500 & -0.38790000 \\
\hline $\mathrm{Cl}$ & -4.90896000 & -3.17594200 & 0.97996100 \\
\hline $\mathrm{Cl}$ & -1.60072400 & -1.66552900 & 1.15667000 \\
\hline $\mathrm{Cl}$ & -4.56424000 & 0.44773800 & 1.06361200 \\
\hline $\mathrm{Cl}$ & -2.69651000 & -3.32602500 & -1.65332700 \\
\hline Mg & -0.66694100 & -3.55241100 & -0.20188100 \\
\hline $\mathrm{Cl}$ & -5.66001000 & -1.21275800 & -1.74636900 \\
\hline Mg & -3.63039000 & -1.43911100 & -0.29482900 \\
\hline $\mathrm{Cl}$ & -2.35179800 & 0.29764300 & -1.56966400 \\
\hline Mg & -0.32167200 & 0.07117600 & -0.11802900 \\
\hline $\mathrm{Cl}$ & 1.36267500 & -3.77873600 & 1.24969700 \\
\hline $\mathrm{Cl}$ & 4.67097700 & -2.26832700 & 1.42651300 \\
\hline $\mathrm{Cl}$ & 1.81882000 & -0.09454900 & 1.30559300 \\
\hline $\mathrm{Cl}$ & 3.57532600 & -3.92887100 & -1.38352400 \\
\hline Mg & 5.60485700 & -4.15527100 & 0.06793800 \\
\hline $\mathrm{Cl}$ & 0.61176400 & -1.81560700 & -1.47665200 \\
\hline Mg & 2.64129100 & -2.04210800 & -0.02531400 \\
\hline $\mathrm{Cl}$ & 3.94387900 & -0.32584800 & -1.42377500 \\
\hline Mg & 5.94908600 & -0.53157500 & 0.15150600 \\
\hline $\mathrm{Cl}$ & 7.97921400 & -0.75797600 & 1.60307300 \\
\hline $\mathrm{Cl}$ & 6.88346800 & -2.41844300 & -1.20690600 \\
\hline $\mathrm{Ti}$ & 2.99184100 & 1.80283300 & -0.18171900 \\
\hline $\mathrm{Cl}$ & 5.18072400 & 1.64985100 & 0.95900000 \\
\hline $\mathrm{Cl}$ & 0.99538200 & 1.69865000 & -1.54881300 \\
\hline C & -3.85069400 & 5.16276600 & -0.87513500 \\
\hline C & -2.75374100 & 4.45325400 & -1.37430600 \\
\hline C & -2.10016400 & 3.51971400 & -0.56965700 \\
\hline C & -2.54258700 & 3.29962200 & 0.74428100 \\
\hline C & -3.64151900 & 4.01885100 & 1.24822200 \\
\hline C & -4.29348500 & 4.94602400 & 0.43734000 \\
\hline $\mathrm{H}$ & -5.15082100 & 5.50170800 & 0.82478700 \\
\hline $\mathrm{H}$ & -4.36698500 & 5.88701200 & -1.51059900 \\
\hline $\mathrm{H}$ & -2.41218800 & 4.61545500 & -2.39891600 \\
\hline $\mathrm{H}$ & -1.26107100 & 2.94633500 & -0.95940900 \\
\hline $\mathrm{H}$ & -3.98394000 & 3.82956300 & 2.26584000 \\
\hline C & -1.87403100 & 2.26849600 & 1.56269100 \\
\hline 0 & -2.28977900 & 2.19730600 & 2.80698500 \\
\hline C & -1.78934500 & 1.12022900 & 3.64100500 \\
\hline C & -2.55570000 & 1.16724900 & 4.94267000 \\
\hline
\end{tabular}




$\begin{array}{lrrr}\text { H } & -1.94170500 & 0.17357500 & 3.10483400 \\ \text { H } & -0.70501300 & 1.26152800 & 3.77425200 \\ \text { H } & -3.63081500 & 1.02271800 & 4.75740200 \\ \text { H } & -2.41124300 & 2.13023200 & 5.45604600 \\ \text { H } & -2.20545200 & 0.36237400 & 5.60668500 \\ \text { O } & -0.98019100 & 1.52545900 & 1.14066400 \\ \text { C } & 3.67218100 & 3.54093300 & -1.28598400 \\ \text { H } & 2.96170500 & 3.99281000 & -1.99338800 \\ \text { H } & 3.90375200 & 2.52781500 & -1.73885900 \\ \text { C } & 4.98743300 & 4.32049000 & -1.18829900 \\ \text { C } & 6.00880300 & 3.76769400 & -2.19734600 \\ \text { H } & 6.96341200 & 4.31383700 & -2.12937900 \\ \text { H } & 5.63732000 & 3.85968600 & -3.23210200 \\ \text { H } & 6.21782800 & 2.70405400 & -2.00047500 \\ \text { C } & 2.14073700 & 3.08407800 & 1.38824100 \\ \text { H } & 2.77697000 & 2.78075200 & 2.22575600 \\ \text { H } & 1.08915000 & 2.78460700 & 1.43304000 \\ \text { C } & 2.51426500 & 4.17396400 & 0.59099200 \\ \text { H } & 3.44425400 & 4.68294600 & 0.85487500 \\ \text { C } & 1.46416900 & 5.00104800 & -0.10435600 \\ \text { H } & 1.87858300 & 5.66374500 & -0.87454800 \\ \text { H } & 0.70203500 & 4.35619300 & -0.56133300 \\ \text { H } & 0.96739900 & 5.63113300 & 0.65342200 \\ \text { H } & 5.41293500 & 4.15080600 & -0.18494500 \\ \text { C } & 4.78876700 & 5.82795800 & -1.38792400 \\ \text { H } & 4.11693200 & 6.26718800 & -0.63468100 \\ \text { H } & 5.75102200 & 6.35911600 & -1.31671600 \\ \text { H } & 4.36125700 & 6.04008900 & -2.38296500\end{array}$

$\mathrm{MgCl}_{2}$-Ti-i sobutyl-EB-Mg1-Propylene-2,1-re-coordination

$\begin{array}{lrrr}\text { Cl } & -7.75462000 & -1.36571600 & 0.77365500 \\ \text { Mg } & -6.73254100 & -3.13268900 & -0.67928400 \\ \text { Cl } & -8.54154500 & 0.72813400 & -1.84313100 \\ \text { Mg } & -6.52471300 & 0.48968500 & -0.37578900 \\ \text { Cl } & -4.71571100 & -3.37113900 & 0.78805700 \\ \text { Cl } & -1.46894600 & -1.75419000 & 1.10597600 \\ \text { Cl } & -4.50788300 & 0.25123300 & 1.09155100 \\ \text { Cl } & -2.46372700 & -3.28270700 & -1.81432900 \\ \text { Mg } & -0.44694500 & -3.52114800 & -0.34702500 \\ \text { Cl } & -5.50263600 & -1.27728600 & -1.82873000 \\ \text { Mg } & -3.48580700 & -1.51573300 & -0.36138400 \\ \text { Cl } & -2.25591100 & 0.33964800 & -1.51082000 \\ \text { Mg } & -0.23874300 & 0.10118400 & -0.04338400 \\ \text { Cl } & 1.56986000 & -3.75955900 & 1.12035500\end{array}$




\begin{tabular}{|c|c|c|c|}
\hline $\mathrm{Cl}$ & 4.81667100 & -2.14260800 & 1.43835100 \\
\hline $\mathrm{Cl}$ & 1.93449100 & 0.01063700 & 1.33333700 \\
\hline $\mathrm{Cl}$ & 3.82202200 & -3.67117300 & -1.48196500 \\
\hline Mg & 5.83874700 & -3.90963200 & -0.01468706 \\
\hline $\mathrm{Cl}$ & 0.78307100 & -1.66573200 & -1.49645706 \\
\hline Mg & 2.79979000 & -1.90430500 & -0.02927006 \\
\hline $\mathrm{Cl}$ & 4.09397800 & -0.11790700 & -1.3773730 \\
\hline Mg & 6.04623300 & -0.28726000 & 0.2887560 \\
\hline $\mathrm{Cl}$ & 8.06342200 & -0.52571500 & 1.75612906 \\
\hline $\mathrm{Cl}$ & 7.06867700 & -2.05420500 & -1.1641470 \\
\hline Ti & 3.20997200 & 2.02618000 & -0.35282706 \\
\hline $\mathrm{Cl}$ & 5.21133300 & 1.87500200 & 1.04871406 \\
\hline $\mathrm{Cl}$ & 0.95779500 & 1.99572200 & -1.22962206 \\
\hline C & -3.96430600 & 5.15739700 & -0.37553506 \\
\hline C & -2.85740000 & 4.50638700 & -0.92919106 \\
\hline C & -2.20349700 & 3.50457800 & -0.2111300 \\
\hline C & -2.65530400 & 3.15693500 & 1.07121200 \\
\hline C & -3.76316100 & 3.81857300 & $1.6315620 e$ \\
\hline C & -4.41589600 & 4.81375500 & 0.90641906 \\
\hline $\mathrm{H}$ & -5.28061700 & 5.32418300 & 1.33734700 \\
\hline $\mathrm{H}$ & -4.48046600 & 5.93566400 & -0.94369506 \\
\hline $\mathrm{H}$ & -2.50625100 & 4.76920600 & -1.92945100 \\
\hline $\mathrm{H}$ & -1.35125500 & 2.98338000 & -0.64148506 \\
\hline $\mathrm{H}$ & -4.11229800 & 3.53243900 & $2.6239460 e$ \\
\hline C & -1.98309700 & 2.06094300 & 1.79883300 \\
\hline 0 & -2.37733200 & 1.91135700 & 3.04675300 \\
\hline C & -1.88649300 & 0.76720600 & 3.78870900 \\
\hline C & -2.57596000 & 0.77289400 & 5.13367900 \\
\hline $\mathrm{H}$ & -2.11318600 & -0.13820100 & 3.20892506 \\
\hline H & -0.79077200 & 0.84711300 & 3.86993300 \\
\hline $\mathrm{H}$ & -3.66581900 & 0.68910400 & 5.00599200 \\
\hline $\mathrm{H}$ & -2.35633400 & 1.69736400 & 5.68958600 \\
\hline $\mathrm{H}$ & -2.22907000 & -0.08386500 & 5.7312460 \\
\hline 0 & -1.10568200 & 1.34259100 & 1.31563000 \\
\hline C & 3.81047400 & 3.17558500 & -1.96567700 \\
\hline $\mathrm{H}$ & 4.75620500 & 3.51193500 & -1.48754000 \\
\hline $\mathrm{H}$ & 4.09091400 & 2.44011300 & -2.74140200 \\
\hline C & 2.99768400 & 4.34095000 & -2.55969200 \\
\hline C & 2.35543100 & 3.93397200 & -3.89095700 \\
\hline C & 3.85172400 & 5.60738200 & -2.71280000 \\
\hline $\mathrm{H}$ & 2.16617600 & 4.58637500 & -1.87313000 \\
\hline $\mathrm{H}$ & 1.71461900 & 4.73910100 & -4.28665300 \\
\hline $\mathrm{H}$ & 1.73543200 & 3.03351400 & $-3.7686280 e$ \\
\hline $\mathrm{H}$ & 3.13081300 & 3.71700200 & -4.6457420 \\
\hline
\end{tabular}




$\begin{array}{llll}\text { H } & 3.25977300 & 6.44334600 & -3.12193400 \\ \text { H } & 4.69945200 & 5.42673800 & -3.39522900 \\ \text { H } & 4.26792600 & 5.93151700 & -1.74410200 \\ \text { C } & 2.45779700 & 3.58324200 & 1.89112400 \\ \text { H } & 3.15385700 & 3.06335000 & 2.56119000 \\ \text { C } & 2.97721300 & 4.33889700 & 0.89984400 \\ \text { H } & 2.32900500 & 4.90520500 & 0.22453400 \\ \text { H } & 4.05526300 & 4.49256400 & 0.80971300 \\ \text { C } & 1.00756000 & 3.44181900 & 2.21080100 \\ \text { H } & 0.75669700 & 2.38816500 & 2.38683300 \\ \text { H } & 0.78597400 & 3.99181100 & 3.14274500 \\ \text { H } & 0.36409800 & 3.82959500 & 1.40932300\end{array}$

$\mathrm{MgCl}_{2}$-Ti-isobutyl-EB-Mg1-Propylene-2,1-re-insertion-TS

$\begin{array}{lrrr}\text { Cl } & -7.76480100 & -1.31606100 & 0.83292100 \\ \text { Mg } & -6.76874300 & -3.11288100 & -0.60136300 \\ \text { Cl } & -8.55884800 & 0.74331400 & -1.80895100 \\ \text { Mg } & -6.53111100 & 0.51203300 & -0.35555800 \\ \text { Cl } & -4.74100700 & -3.34416300 & 0.85202900 \\ \text { Cl } & -1.47958500 & -1.74734300 & 1.11694400 \\ \text { Cl } & -4.50337500 & 0.28075000 & 1.09783200 \\ \text { Cl } & -2.51125900 & -3.31288500 & -1.77074000 \\ \text { Mg } & -0.48361700 & -3.54415900 & -0.31743200 \\ \text { Cl } & -5.53505400 & -1.28478500 & -1.78984600 \\ \text { Mg } & -3.50732600 & -1.51605400 & -0.33643900 \\ \text { Cl } & -2.27364100 & 0.31205300 & -1.52491100 \\ \text { Mg } & -0.24513500 & 0.08063200 & -0.07141400 \\ \text { Cl } & 1.54414900 & -3.77542200 & 1.13602500 \\ \text { Cl } & 4.80565000 & -2.17861200 & 1.40104200 \\ \text { Cl } & 1.93879800 & 0.00244200 & 1.33357400 \\ \text { Cl } & 3.77408000 & -3.74414700 & -1.48667400 \\ \text { Mg } & 5.80172200 & -3.97545800 & -0.03331100 \\ \text { Cl } & 0.75025400 & -1.71600300 & -1.50585500 \\ \text { Mg } & 2.77768100 & -1.94752200 & -0.05234700 \\ \text { Cl } & 4.01323200 & -0.15377600 & -1.38266100 \\ \text { Mg } & 6.03850600 & -0.35051200 & 0.21213500 \\ \text { Cl } & 8.06707400 & -0.58185900 & 1.66585400 \\ \text { Cl } & 7.03562800 & -2.14729500 & -1.22173000 \\ \text { Ti } & 3.09782400 & 1.97942500 & -0.14077200 \\ \text { Cl } & 5.39054100 & 1.87314000 & 0.85438900 \\ \text { Cl } & 1.00349500 & 1.87742600 & -1.35049900 \\ \text { C } & -3.86463900 & 5.17541800 & -0.54756300 \\ \text { C } & -2.76167500 & 4.49477100 & -1.07288500 \\ \text { C } & -2.13219300 & 3.49983500 & -0.32435500\end{array}$




\begin{tabular}{|c|c|c|c|}
\hline C & -2.60402500 & 3.18919100 & 0.96021500 \\
\hline C & -3.70775200 & 3.88025900 & 1.49181200 \\
\hline C & -4.33668800 & 4.86824200 & 0.73614500 \\
\hline $\mathrm{H}$ & -5.19846100 & 5.40147100 & 1.14475800 \\
\hline $\mathrm{H}$ & -4.36205100 & 5.94800800 & -1.13967200 \\
\hline $\mathrm{H}$ & -2.39567400 & 4.72862900 & -2.07503400 \\
\hline $\mathrm{H}$ & -1.28484600 & 2.95370200 & -0.73336200 \\
\hline $\mathrm{H}$ & -4.07189700 & 3.62257300 & 2.48663400 \\
\hline C & -1.95638800 & 2.10105300 & 1.72078400 \\
\hline 0 & -2.36197300 & 1.98831200 & 2.96793600 \\
\hline C & -1.89285800 & 0.85624900 & 3.74225700 \\
\hline C & -2.62283200 & 0.88231900 & 5.06537600 \\
\hline $\mathrm{H}$ & -2.10171400 & -0.05860200 & 3.17086900 \\
\hline $\mathrm{H}$ & -0.80021900 & 0.94065600 & 3.85464600 \\
\hline $\mathrm{H}$ & -3.70792100 & 0.79284900 & 4.90500100 \\
\hline $\mathrm{H}$ & -2.42242900 & 1.81639700 & 5.61232300 \\
\hline H & -2.29229100 & 0.03646000 & 5.68725700 \\
\hline 0 & -1.08957300 & 1.35541300 & 1.26003200 \\
\hline C & 3.75826500 & 3.64383600 & -1.33673600 \\
\hline $\mathrm{H}$ & 4.79327400 & 3.99681900 & -1.21985700 \\
\hline $\mathrm{H}$ & 3.86366100 & 2.62094500 & -1.81546400 \\
\hline C & 2.92502100 & 4.54619200 & -2.25293300 \\
\hline C & 2.90855500 & 3.99198900 & -3.68737600 \\
\hline C & 3.43300200 & 5.99362700 & -2.23216500 \\
\hline $\mathrm{H}$ & 1.88119700 & 4.53175700 & -1.89365000 \\
\hline $\mathrm{H}$ & 2.31107800 & 4.63932200 & -4.34986900 \\
\hline $\mathrm{H}$ & 2.46786600 & 2.98363500 & -3.71189500 \\
\hline $\mathrm{H}$ & 3.92974900 & 3.93678400 & -4.10112500 \\
\hline $\mathrm{H}$ & 2.79277300 & 6.64391200 & -2.84926600 \\
\hline H & 4.45825800 & 6.05046500 & -2.63565100 \\
\hline $\mathrm{H}$ & 3.45564300 & 6.41612800 & -1.21525600 \\
\hline C & 2.61307400 & 3.35432200 & 1.61382100 \\
\hline $\mathrm{H}$ & 3.26664100 & 2.86720800 & 2.34730700 \\
\hline C & 3.19864200 & 4.30158400 & 0.77800900 \\
\hline $\mathrm{H}$ & 2.56410900 & 5.05258000 & 0.30318900 \\
\hline $\mathrm{H}$ & 4.24984100 & 4.56290800 & 0.90087100 \\
\hline C & 1.13791400 & 3.37625000 & 1.93711800 \\
\hline $\mathrm{H}$ & 0.79892000 & 2.39696000 & 2.29084700 \\
\hline $\mathrm{H}$ & 0.93928400 & 4.11286200 & 2.73632100 \\
\hline $\mathrm{H}$ & 0.52858100 & 3.64950800 & 1.064299 \\
\hline
\end{tabular}

$\mathrm{MgCl}_{2}$-Ti-isobutyl-EB-Mg1-Propylene-2,1-si-coordination
Cl $\quad-7.79036100$
$-1.32794200$
0.77905500
$\mathrm{Mg}$
$-6.74440700$
$-3.12155000$
$-0.62337800$ 


\begin{tabular}{|c|c|c|c|}
\hline $\mathrm{Cl}$ & -8.57804900 & 0.69386200 & -1.89356300 \\
\hline Mg & -6.56866100 & 0.50884800 & -0.40840500 \\
\hline $\mathrm{Cl}$ & -4.73502100 & -3.30656600 & 0.86178000 \\
\hline $\mathrm{Cl}$ & -1.50391800 & -1.65479800 & 1.15948500 \\
\hline $\mathrm{Cl}$ & -4.55927600 & 0.32383100 & 1.07675200 \\
\hline $\mathrm{Cl}$ & -2.46737000 & -3.26338300 & -1.72811500 \\
\hline Mg & -0.45803600 & -3.44839100 & -0.2430000 \\
\hline $\mathrm{Cl}$ & -5.52270900 & -1.28475900 & -1.81083900 \\
\hline Mg & -3.51332700 & -1.46976700 & -0.32567200 \\
\hline $\mathrm{Cl}$ & -2.29164100 & 0.36700300 & -1.51312200 \\
\hline Mg & -0.28189100 & 0.18197700 & -0.02787300 \\
\hline $\mathrm{Cl}$ & 1.55132600 & -3.63336500 & 1.24220000 \\
\hline $\mathrm{Cl}$ & 4.78248000 & -1.98158700 & 1.53999400 \\
\hline $\mathrm{Cl}$ & 1.86514200 & 0.09939000 & 1.35986600 \\
\hline $\mathrm{Cl}$ & 3.81915800 & -3.59022400 & -1.34762700 \\
\hline Mg & 5.82843700 & -3.77525200 & 0.13746700 \\
\hline $\mathrm{Cl}$ & 0.76376600 & -1.61160400 & -1.43043200 \\
\hline Mg & 2.77305600 & -1.79673200 & 0.05455700 \\
\hline $\mathrm{Cl}$ & 4.06791400 & -0.05502300 & -1.34012400 \\
\hline Mg & 6.00384500 & -0.14484700 & 0.35238600 \\
\hline $\mathrm{Cl}$ & 8.01358500 & -0.32987300 & 1.83757700 \\
\hline $\mathrm{Cl}$ & 7.05016200 & -1.93843500 & -1.05000900 \\
\hline $\mathrm{Ti}$ & 3.13884000 & 2.07621300 & -0.38741100 \\
\hline $\mathrm{Cl}$ & 5.05465400 & 2.00267700 & 1.10816300 \\
\hline $\mathrm{Cl}$ & 0.91653000 & 1.98407600 & -1.35739300 \\
\hline C & -4.08859200 & 5.09251300 & -0.57149300 \\
\hline C & -2.92537600 & 4.50109200 & -1.07436500 \\
\hline C & -2.22478700 & 3.57181400 & -0.30514800 \\
\hline C & -2.68796800 & 3.23774700 & 0.97696100 \\
\hline C & -3.85202100 & 3.84115400 & 1.48732300 \\
\hline C & -4.55032600 & 4.76383700 & 0.71110900 \\
\hline $\mathrm{H}$ & -5.45847800 & 5.22918300 & 1.10190600 \\
\hline $\mathrm{H}$ & -4.64113200 & 5.81316400 & -1.18001700 \\
\hline $\mathrm{H}$ & -2.56686400 & 4.75320300 & -2.07471200 \\
\hline $\mathrm{H}$ & -1.32647800 & 3.09626800 & -0.69484200 \\
\hline $\mathrm{H}$ & -4.20881800 & 3.56394300 & 2.47955400 \\
\hline C & -1.96899200 & 2.20771900 & 1.75089100 \\
\hline 0 & -2.37882900 & 2.06190500 & 2.99182900 \\
\hline C & -1.81518000 & 0.98233600 & 3.77986000 \\
\hline C & -2.51941700 & 0.98563500 & 5.11705000 \\
\hline $\mathrm{H}$ & -1.96862400 & 0.04274500 & 3.23120200 \\
\hline $\mathrm{H}$ & -0.72905400 & 1.14579900 & 3.86620400 \\
\hline $\mathrm{H}$ & -3.59904800 & 0.82058800 & 4.982138 \\
\hline $\mathrm{H}$ & -2.37202300 & 1.94174300 & 5.6423640 \\
\hline
\end{tabular}




$\begin{array}{lrrr}\text { H } & -2.11998600 & 0.17643700 & 5.74717900 \\ \text { O } & -1.03698700 & 1.53004800 & 1.30136800 \\ \text { C } & 3.94890300 & 3.23090400 & -1.90491700 \\ \text { H } & 4.00309400 & 4.20542000 & -1.37429800 \\ \text { H } & 4.98934000 & 2.85297100 & -1.96643900 \\ \text { C } & 3.33477800 & 3.39521500 & -3.30065200 \\ \text { C } & 3.30646600 & 2.07131500 & -4.07301000 \\ \text { C } & 4.07915600 & 4.47859500 & -4.09834300 \\ \text { H } & 2.29026300 & 3.72987200 & -3.16798500 \\ \text { H } & 2.88002600 & 2.21233800 & -5.07928900 \\ \text { H } & 2.70218600 & 1.31631300 & -3.55071300 \\ \text { H } & 4.32557300 & 1.66501500 & -4.19139200 \\ \text { H } & 3.61517800 & 4.63392400 & -5.08697500 \\ \text { H } & 5.13114700 & 4.18834000 & -4.26202300 \\ \text { H } & 4.07793500 & 5.44479000 & -3.56748000 \\ \text { C } & 1.82066000 & 3.42528200 & 1.79837200 \\ \text { H } & 0.86033700 & 2.95314200 & 1.56107600 \\ \text { C } & 2.36557200 & 4.24910100 & 0.87968500 \\ \text { H } & 1.82407700 & 4.48991100 & -0.04085400 \\ \text { H } & 3.30988900 & 4.76921800 & 1.06904700 \\ \text { C } & 2.40357600 & 3.11178500 & 3.13829800 \\ \text { H } & 1.72424100 & 3.48409300 & 3.92554100 \\ \text { H } & 2.48826700 & 2.02294800 & 3.27567400 \\ \text { H } & 3.39578900 & 3.56288000 & 3.27783800\end{array}$

$\mathrm{MgCl}_{2}$-Ti-isobutyl-EB-Mg1-Propylene-2,1-si-insertion-TS

$\begin{array}{lrrr}\text { Cl } & -7.77426300 & -1.28033600 & 0.90032300 \\ \text { Mg } & -6.79481100 & -3.09872300 & -0.51812900 \\ \text { Cl } & -8.59212900 & 0.74238700 & -1.76251800 \\ \text { Mg } & -6.55025300 & 0.52898600 & -0.32628400 \\ \text { Cl } & -4.75293700 & -3.31212600 & 0.91810500 \\ \text { Cl } & -1.48708900 & -1.71620600 & 1.12769700 \\ \text { Cl } & -4.50838100 & 0.31558300 & 1.10994900 \\ \text { Cl } & -2.54947800 & -3.32119100 & -1.72695600 \\ \text { Mg } & -0.50767000 & -3.53458900 & -0.29078300 \\ \text { Cl } & -5.57080300 & -1.28940100 & -1.74473700 \\ \text { Mg } & -3.52893100 & -1.50279900 & -0.30849800 \\ \text { Cl } & -2.30491800 & 0.30651600 & -1.53511700 \\ \text { Mg } & -0.26252700 & 0.09311200 & -0.09860600 \\ \text { Cl } & 1.53417800 & -3.74794100 & 1.14550200 \\ \text { Cl } & 4.80016400 & -2.15202000 & 1.35531800 \\ \text { Cl } & 1.89384100 & -0.05246100 & 1.30927000 \\ \text { Cl } & 3.73784000 & -3.75705000 & -1.49948700 \\ \text { Mg } & 5.77962100 & -3.97047700 & -0.06328600\end{array}$




\begin{tabular}{|c|c|c|c|}
\hline $\mathrm{Cl}$ & 0.71648000 & -1.72525300 & -1.51735300 \\
\hline Mg & 2.75841100 & -1.93865500 & -0.08124800 \\
\hline $\mathrm{Cl}$ & 3.99937600 & -0.14893900 & -1.42798800 \\
\hline Mg & 6.02322200 & -0.34302400 & 0.12827100 \\
\hline $\mathrm{Cl}$ & 8.06606700 & -0.55613900 & 1.56472100 \\
\hline $\mathrm{Cl}$ & 7.00385000 & -2.16103700 & -1.28989200 \\
\hline $\mathrm{Ti}$ & 3.01212400 & 1.93100700 & -0.12215400 \\
\hline $\mathrm{Cl}$ & 5.22754000 & 1.82379900 & 0.95373800 \\
\hline $\mathrm{Cl}$ & 1.01467200 & 1.78161300 & -1.48995700 \\
\hline C & -3.83459900 & 5.12431600 & -0.74069200 \\
\hline C & -2.68153600 & 4.49000400 & -1.21414000 \\
\hline C & -2.03018000 & 3.54318900 & -0.42355200 \\
\hline C & -2.53096800 & 3.23594700 & 0.85119000 \\
\hline C & -3.68502400 & 3.88111000 & 1.33132500 \\
\hline C & -4.33521200 & 4.82053000 & 0.53312000 \\
\hline $\mathrm{H}$ & -5.23580600 & 5.31860100 & 0.90033600 \\
\hline $\mathrm{H}$ & -4.34937000 & 5.85830900 & -1.36611700 \\
\hline $\mathrm{H}$ & -2.29411900 & 4.72149900 & -2.20872900 \\
\hline $\mathrm{H}$ & -1.14176600 & 3.03226000 & -0.79140200 \\
\hline $\mathrm{H}$ & -4.07063800 & 3.62456000 & 2.31835100 \\
\hline C & -1.85934200 & 2.19319300 & 1.65065000 \\
\hline 0 & -2.29110000 & 2.07971700 & 2.88593600 \\
\hline C & -1.78380200 & 0.98767000 & 3.69591600 \\
\hline C & -2.55454900 & 0.99717300 & 4.99578500 \\
\hline $\mathrm{H}$ & -1.92583800 & 0.05284700 & 3.13667200 \\
\hline $\mathrm{H}$ & -0.70116700 & 1.13573700 & 3.83591100 \\
\hline $\mathrm{H}$ & -3.62759600 & 0.84622700 & 4.80387700 \\
\hline $\mathrm{H}$ & -2.42122100 & 1.94968200 & 5.53118900 \\
\hline $\mathrm{H}$ & -2.19840800 & 0.18084100 & 5.64245900 \\
\hline 0 & -0.94687600 & 1.47659800 & 1.22126300 \\
\hline C & 3.65093800 & 3.70594100 & -1.17401700 \\
\hline $\mathrm{H}$ & 4.58818100 & 4.15124800 & -0.80722600 \\
\hline $\mathrm{H}$ & 3.95671300 & 2.71298800 & -1.62892400 \\
\hline C & 2.98713600 & 4.54373700 & -2.26765400 \\
\hline C & 3.49238400 & 4.10614700 & -3.65336800 \\
\hline C & 3.21269200 & 6.04564900 & -2.05382300 \\
\hline $\mathrm{H}$ & 1.90250500 & 4.33915600 & -2.24534800 \\
\hline $\mathrm{H}$ & 3.02027500 & 4.70540800 & -4.44881400 \\
\hline $\mathrm{H}$ & 3.25480200 & 3.04724700 & -3.84244700 \\
\hline $\mathrm{H}$ & 4.58481000 & 4.23512200 & -3.73688900 \\
\hline $\mathrm{H}$ & 2.70278000 & 6.63252700 & -2.83425700 \\
\hline $\mathrm{H}$ & 4.28823100 & 6.28762800 & -2.10126500 \\
\hline $\mathrm{H}$ & 2.83585500 & 6.39275200 & -1.07957900 \\
\hline C & 2.05405200 & 3.17417900 & 1.50171600 \\
\hline
\end{tabular}




$\begin{array}{lrrr}\text { H } & 1.05258300 & 2.74200400 & 1.39429700 \\ \text { C } & 2.37815600 & 4.20663200 & 0.62311300 \\ \text { H } & 1.62436600 & 4.56544400 & -0.07901500 \\ \text { H } & 3.16225900 & 4.91797100 & 0.89244000 \\ \text { C } & 2.72529300 & 2.98524800 & 2.84502700 \\ \text { H } & 2.12727500 & 3.48139600 & 3.63057700 \\ \text { H } & 2.81072300 & 1.92308400 & 3.11452600 \\ \text { H } & 3.73611400 & 3.41628100 & 2.85784200\end{array}$

$\begin{array}{llll}\mathrm{MgCl}_{2} \text {-Ti-isobutyl-CMDMS-Mg2-Propylene-1,2-re-coordination } \\ \mathrm{Cl} & 7.04079300 & -2.28691200 & -1.42707900 \\ \mathrm{Mg} & 5.95003300 & -4.00891300 & 0.02997000 \\ \mathrm{Cl} & 8.17639500 & -0.37505200 & 1.20090500 \\ \mathrm{Mg} & 6.06047800 & -0.37362700 & -0.14092200 \\ \mathrm{Cl} & 3.83402000 & -4.00748300 & -1.31168500 \\ \mathrm{Cl} & 0.73762800 & -2.09271000 & -1.36711100 \\ \mathrm{Cl} & 3.94438000 & -0.37215300 & -1.48240900 \\ \mathrm{Cl} & 1.76292400 & -3.81612100 & 1.43159900 \\ \mathrm{Mg} & -0.35312400 & -3.81469100 & 0.08991500 \\ \mathrm{Cl} & 4.96968500 & -2.09553500 & 1.31627400 \\ \mathrm{Mg} & 2.85367900 & -2.09415200 & -0.02544900 \\ \mathrm{Cl} & 1.87332200 & -0.18082100 & 1.26081400 \\ \mathrm{Mg} & -0.24246800 & -0.17961000 & -0.08083700 \\ \mathrm{Cl} & -2.46904700 & -3.81323100 & -1.25171500 \\ \mathrm{Cl} & -5.56547700 & -1.89849000 & -1.30715400 \\ \mathrm{Cl} & -2.37172000 & -0.12123800 & -1.45452600 \\ \mathrm{Cl} & -4.54028400 & -3.62190800 & 1.49149300 \\ \mathrm{Mg} & -6.65617700 & -3.62047100 & 0.14993300 \\ \mathrm{Cl} & -1.33343200 & -1.90138100 & 1.37626300 \\ \mathrm{Mg} & -3.44932000 & -1.89947500 & 0.03467500 \\ \mathrm{Cl} & -4.37941300 & 0.10662200 & 1.36961800 \\ \mathrm{Mg} & -6.54617100 & 0.01467400 & -0.02099600 \\ \mathrm{Cl} & -8.66185600 & 0.01626800 & -1.36248400 \\ \mathrm{Cl} & -7.63650800 & -1.70712200 & 1.43621600 \\ \mathrm{Ti} & -3.34637400 & 2.05043200 & -0.01391300 \\ \mathrm{Cl} & -5.47105700 & 1.95224800 & -1.15098400 \\ \mathrm{Cl} & -0.96348800 & 1.96694700 & 0.73609800 \\ \mathrm{C} & 3.88879900 & 3.15306800 & -0.40533300 \\ \mathrm{C} & 2.96816900 & 3.46692900 & 0.81001500 \\ \mathrm{C} & 3.92515700 & 4.34866100 & -1.37578900 \\ \mathrm{H} & 3.45906700 & 2.28779200 & -0.94011900 \\ \mathrm{C} & 1.78700600 & 4.38642300 & 0.42018300 \\ \mathrm{H} & 3.53649000 & 3.94809600 & 1.62753900 \\ & & & \\ & & & \\ & & & \end{array}$




\begin{tabular}{|c|c|c|c|}
\hline $\mathrm{H}$ & 2.58778800 & 2.51548400 & 1.21063600 \\
\hline C & 2.52684100 & 4.58695600 & -1.99730200 \\
\hline $\mathrm{H}$ & 4.25089200 & 5.24916400 & -0.82542600 \\
\hline $\mathrm{H}$ & 4.66520500 & 4.20283300 & -2.18008200 \\
\hline C & 1.38747400 & 4.16923900 & -1.04178700 \\
\hline $\mathrm{H}$ & 2.06571900 & 5.44644500 & 0.55705500 \\
\hline $\mathrm{H}$ & 0.93261000 & 4.19710200 & 1.08834300 \\
\hline $\mathrm{H}$ & 2.43973700 & 4.02269100 & -2.94095000 \\
\hline $\mathrm{H}$ & 2.42351200 & 5.65272200 & -2.26349700 \\
\hline $\mathrm{H}$ & 1.14858300 & 3.10059500 & -1.18068900 \\
\hline $\mathrm{H}$ & 0.46352800 & 4.72055500 & -1.28455100 \\
\hline C & 6.72182600 & 3.65029900 & 0.99571400 \\
\hline $\mathrm{H}$ & 7.58669600 & 3.06359900 & 1.34532700 \\
\hline $\mathrm{H}$ & 7.07454100 & 4.41283300 & 0.28246100 \\
\hline $\mathrm{H}$ & 6.26815700 & 4.17289900 & 1.85264200 \\
\hline 0 & 5.23926500 & 1.12667400 & 1.14687100 \\
\hline 0 & 6.32664400 & 1.57065100 & -1.02166800 \\
\hline C & 5.22741300 & 1.03084200 & 2.57444900 \\
\hline $\mathrm{H}$ & 4.36989100 & 0.40892900 & 2.86530200 \\
\hline $\mathrm{H}$ & 6.17139100 & 0.56556000 & 2.89601200 \\
\hline $\mathrm{H}$ & 5.11907400 & 2.02632000 & 3.03351000 \\
\hline C & 6.42468300 & 1.82508300 & -2.41841800 \\
\hline $\mathrm{H}$ & 5.44353000 & 1.70182300 & -2.90443600 \\
\hline $\mathrm{H}$ & 6.81719900 & 2.83754800 & -2.60942500 \\
\hline $\mathrm{H}$ & 7.12388300 & 1.09102600 & -2.84372100 \\
\hline $\mathrm{Si}$ & 5.53024700 & 2.47988400 & 0.18051100 \\
\hline C & -3.61601400 & 3.49044200 & 1.43939000 \\
\hline $\mathrm{H}$ & -4.57576800 & 3.86227600 & 1.01971900 \\
\hline $\mathrm{H}$ & -3.87748300 & 2.85970800 & 2.31150700 \\
\hline C & -2.64894700 & 4.62473200 & 1.82626200 \\
\hline C & -1.97613500 & 4.34212600 & 3.17478900 \\
\hline C & -3.34987400 & 5.99057000 & 1.82749100 \\
\hline $\mathrm{H}$ & -1.83722800 & 4.67256500 & 1.07638700 \\
\hline $\mathrm{H}$ & -1.22688200 & 5.11472800 & 3.41269700 \\
\hline $\mathrm{H}$ & -1.46643600 & 3.36726000 & 3.16587800 \\
\hline $\mathrm{H}$ & -2.72294500 & 4.33233600 & 3.98706700 \\
\hline $\mathrm{H}$ & -2.64531300 & 6.80037000 & 2.07957000 \\
\hline $\mathrm{H}$ & -4.16759500 & 6.01022200 & 2.56738300 \\
\hline $\mathrm{H}$ & -3.78821900 & 6.22133300 & 0.84209600 \\
\hline C & -2.35846500 & 3.15031300 & -2.16853100 \\
\hline $\mathrm{H}$ & -2.87339200 & 2.56424800 & -2.93683600 \\
\hline $\mathrm{H}$ & -1.33767400 & 2.86131600 & -1.91009200 \\
\hline C & -2.91200200 & 4.26829600 & -1.64727400 \\
\hline $\mathrm{H}$ & -2.32494800 & 4.82926800 & -0.9099180 \\
\hline
\end{tabular}




$\begin{array}{llll}\mathrm{C} & -4.21277100 & 4.88429000 & -2.05543400 \\ \mathrm{H} & -4.86065400 & 5.06594300 & -1.18378400 \\ \mathrm{H} & -4.01055900 & 5.86889300 & -2.51229600 \\ \mathrm{H} & -4.76170100 & 4.26182000 & -2.77426400\end{array}$

$\mathrm{MgCl}_{2}$-Ti-isobuty1-CMDMS-Mg2-Propylene-1,2-re-insertion-TS

\begin{tabular}{|c|c|c|c|}
\hline $\mathrm{Cl}$ & 7.07012100 & -2.23557400 & 800 \\
\hline Mg & 5.99086900 & -3.97087400 & 0.02726500 \\
\hline $\mathrm{Cl}$ & 8.19003000 & -0.32538700 & 1.21336400 \\
\hline Mg & 6.07508100 & -0.33426900 & -0.12995906 \\
\hline $\mathrm{Cl}$ & 3.87582400 & -3.97975400 & -1.31588706 \\
\hline $\mathrm{Cl}$ & 0.76569300 & -2.08725700 & -1.36637106 \\
\hline $\mathrm{Cl}$ & 3.95995000 & -0.34310500 & -1.47294306 \\
\hline $\mathrm{Cl}$ & 1.80151000 & -3.81367000 & 1.42661306 \\
\hline Mg & -0.31352800 & -3.82255300 & 0.0834720 \\
\hline $\mathrm{Cl}$ & 4.99579600 & -2.06947600 & 1.32001106 \\
\hline Mg & 2.88077800 & -2.07837200 & -0.0232410 \\
\hline $\mathrm{Cl}$ & 1.88568200 & -0.17702100 & 1.2694860 \\
\hline Mg & -0.22956600 & -0.18630400 & -0.07361500 \\
\hline $\mathrm{Cl}$ & -2.42852400 & -3.83139700 & $-1.259694 \ell$ \\
\hline $\mathrm{Cl}$ & -5.53871600 & -1.93893700 & -1.3102010 \\
\hline $\mathrm{Cl}$ & -2.34263800 & -0.14410900 & -1.4837550 \\
\hline $\mathrm{Cl}$ & -4.50298600 & -3.66534600 & 1.48273406 \\
\hline Mg & -6.61790600 & -3.67422400 & 0.13967406 \\
\hline $\mathrm{Cl}$ & -1.30858100 & -1.92120500 & 1.37625306 \\
\hline Mg & -3.42341800 & -1.92966100 & 0.03306200 \\
\hline $\mathrm{Cl}$ & -4.32240700 & 0.05026100 & 1.35328106 \\
\hline Mg & -6.53389900 & -0.03765800 & -0.0175500 \\
\hline $\mathrm{Cl}$ & -8.64885100 & -0.04648500 & -1.36057000 \\
\hline $\mathrm{Cl}$ & -7.61297700 & -1.77286400 & 1.43237800 \\
\hline $\mathrm{Ti}$ & -3.26766400 & 1.98987200 & -0.15904300 \\
\hline $\mathrm{Cl}$ & -5.57062400 & 1.99375400 & -1.01332800 \\
\hline $\mathrm{Cl}$ & -1.04749300 & 1.88354800 & 0.89660900 \\
\hline C & 3.83954700 & 3.13737200 & -0.38083100 \\
\hline C & 2.92686900 & 3.47432700 & 0.83300500 \\
\hline C & 3.82420300 & 4.29187700 & -1.39987700 \\
\hline $\mathrm{H}$ & 3.42645500 & 2.24052400 & -0.87442300 \\
\hline C & 1.70166100 & 4.32286000 & 0.41621200 \\
\hline $\mathrm{H}$ & 3.48746500 & 4.02072300 & 1.61386200 \\
\hline $\mathrm{H}$ & 2.59172400 & 2.53000600 & 1.28740400 \\
\hline C & 2.41113300 & 4.44972200 & -2.01451600 \\
\hline $\mathrm{H}$ & 4.12138400 & 5.22627000 & -0.89140800 \\
\hline $\mathrm{H}$ & 4.56003700 & 4.13981200 & $-2.206445 t$ \\
\hline C & 1.29854200 & 4.02589900 & $-1.030451 e$ \\
\hline
\end{tabular}




\begin{tabular}{|c|c|c|c|}
\hline $\mathrm{H}$ & 1.93416300 & 5.39908500 & 0.50375700 \\
\hline $\mathrm{H}$ & 0.86265600 & 4.12506200 & 1.10124500 \\
\hline $\mathrm{H}$ & 2.33724100 & 3.84489400 & -2.93366600 \\
\hline $\mathrm{H}$ & 2.26139100 & 5.49894300 & -2.32122100 \\
\hline $\mathrm{H}$ & 1.10195700 & 2.94314000 & -1.12204500 \\
\hline $\mathrm{H}$ & 0.35110200 & 4.52829000 & -1.28756200 \\
\hline C & 6.66551400 & 3.71348500 & 1.01200000 \\
\hline $\mathrm{H}$ & 7.54691400 & 3.15117100 & 1.35999700 \\
\hline $\mathrm{H}$ & 6.99582300 & 4.48496800 & 0.29755100 \\
\hline $\mathrm{H}$ & 6.20049000 & 4.22431900 & 1.86992900 \\
\hline 0 & 5.24117100 & 1.15331600 & 1.16663900 \\
\hline 0 & 6.31760300 & 1.61971700 & -1.00051200 \\
\hline C & 5.23546100 & 1.05386700 & 2.59395700 \\
\hline $\mathrm{H}$ & 4.38615100 & 0.42153500 & 2.88661800 \\
\hline $\mathrm{H}$ & 6.18571400 & 0.59821800 & 2.91074800 \\
\hline $\mathrm{H}$ & 5.11785900 & 2.04705700 & 3.05586600 \\
\hline C & 6.42979100 & 1.87813700 & -2.39537900 \\
\hline $\mathrm{H}$ & 5.45995200 & 1.72654500 & -2.89573000 \\
\hline $\mathrm{H}$ & 6.79545000 & 2.90168000 & -2.58032800 \\
\hline H & 7.15661500 & 1.16512300 & -2.81010400 \\
\hline Si & 5.50198100 & 2.51276700 & 0.20034200 \\
\hline C & -3.65371000 & 3.87699700 & 0.82767500 \\
\hline $\mathrm{H}$ & -4.61623900 & 4.36953100 & 0.63075200 \\
\hline $\mathrm{H}$ & -3.94052200 & 2.94027300 & 1.40245400 \\
\hline C & -2.72300300 & 4.71748700 & 1.70851400 \\
\hline C & -2.89203600 & 4.33178200 & 3.18794500 \\
\hline C & -2.93927700 & 6.22250500 & 1.50740800 \\
\hline $\mathrm{H}$ & -1.68057700 & 4.47886900 & 1.43610500 \\
\hline $\mathrm{H}$ & -2.21957000 & 4.92675900 & 3.82674700 \\
\hline $\mathrm{H}$ & -2.65277500 & 3.26835900 & 3.34623500 \\
\hline $\mathrm{H}$ & -3.92623800 & 4.50879900 & 3.52862100 \\
\hline $\mathrm{H}$ & -2.25847400 & 6.80173000 & 2.15089200 \\
\hline $\mathrm{H}$ & -3.97254000 & 6.50985100 & 1.76653100 \\
\hline $\mathrm{H}$ & -2.75542500 & 6.53853100 & 0.46908200 \\
\hline C & -2.51290900 & 3.03196200 & -1.94827200 \\
\hline $\mathrm{H}$ & -3.13564900 & 2.64524600 & -2.76236200 \\
\hline $\mathrm{H}$ & -1.46479900 & 2.71991100 & -1.93922100 \\
\hline C & -2.86603900 & 4.22926000 & -1.31310500 \\
\hline $\mathrm{H}$ & -2.06147500 & 4.76387000 & -0.80174300 \\
\hline C & -4.01469400 & 5.06475900 & -1.81135800 \\
\hline $\mathrm{H}$ & -4.34173400 & 5.82136400 & -1.08689900 \\
\hline $\mathrm{H}$ & -3.67136600 & 5.59298900 & -2.71790800 \\
\hline H & -4.87349500 & 4.43794800 & -2.0872320 \\
\hline
\end{tabular}


$\mathrm{MgCl}_{2}$-Ti-isobutyl-CMDMS-Mg2-Propylene-1,2-si-coordination

\begin{tabular}{|c|c|c|c|}
\hline Cl & 7.17421400 & -2.15398900 & \\
\hline Mg & 6.06699100 & -3.90779400 & 0.13127400 \\
\hline Cl & 8.21940700 & -0.26454600 & 1.40664600 \\
\hline & 6.13867000 & -0.26951200 & 0.01089500 \\
\hline & 3.98615700 & -3.91276100 & -1.26430900 \\
\hline 1 & 0.86975300 & -2.03318000 & -1.37440100 \\
\hline $\mathrm{Cl}$ & 4.05774600 & -0.27444100 & -1.38451800 \\
\hline $\mathrm{Cl}$ & 1.84337600 & -3.78202000 & 1.42698400 \\
\hline Mg & -0.23738400 & -3.78699800 & 0.03147800 \\
\hline $\mathrm{Cl}$ & 5.03141400 & -2.02323400 & 1.41683700 \\
\hline $\lg$ & 2.95063300 & -2.02817900 & 0.02112500 \\
\hline $\mathrm{Cl}$ & 1.91497400 & -0.14369300 & 1.30676100 \\
\hline $\mathrm{Mg}$ & -0.16647800 & -0.14881400 & -0.08900200 \\
\hline Cl & -2.31823000 & -3.79191300 & -1.36419200 \\
\hline Cl & -5.43468700 & -1.91235200 & -1.47436000 \\
\hline Cl & -2.26368500 & -0.09638700 & -1.50448400 \\
\hline $\mathrm{Cl}$ & -4.46119200 & -3.66120300 & 1.32702400 \\
\hline $\mathrm{Mg}$ & -6.54193800 & -3.66616500 & -0.06848700 \\
\hline Cl & -1.27302700 & -1.90243600 & 1.31702000 \\
\hline Mg & -3.35378100 & -1.90761000 & -0.07855300 \\
\hline $\mathrm{Cl}$ & -4.35491100 & 0.03083500 & 1.27050700 \\
\hline $\mathrm{Mg}$ & -6.46978200 & -0.02782200 & -0.18870700 \\
\hline $\mathrm{Cl}$ & -8.55113200 & -0.03281600 & -1.58431000 \\
\hline $\mathrm{Cl}$ & -7.57748900 & -1.78162100 & 1.21707800 \\
\hline Ti & -3.31229600 & 2.02441000 & -0.00266900 \\
\hline $\mathrm{Cl}$ & -5.37366900 & 1.94897700 & -1.24144000 \\
\hline $\mathrm{Cl}$ & -0.90715300 & 2.01022300 & 0.72279200 \\
\hline & 4.03095700 & 3.25037200 & -0.44560700 \\
\hline & 2.99447600 & 3.54705200 & 0.67526900 \\
\hline C & 4.14392300 & 4.44944700 & -1.40572900 \\
\hline & 3.66241200 & 2.38410100 & -1.02231500 \\
\hline & 1.84750300 & 4.45549700 & 0.17287600 \\
\hline $\mathrm{H}$ & 3.47402800 & 4.03107900 & 1.54611100 \\
\hline $\mathrm{H}$ & 2.58785100 & 2.58973800 & 1.03558800 \\
\hline & 2.81142400 & 4.65977700 & -2.16447600 \\
\hline & 4.39410800 & 5.35432400 & -0.82409200 \\
\hline & 4.96451000 & 4.32143800 & -2.13129100 \\
\hline & 1.58963100 & 4.23265500 & -1.32023500 \\
\hline & 2.10337200 & 5.51798700 & 0.33275300 \\
\hline 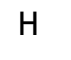 & 0.93716000 & 4.25857400 & 0.76005100 \\
\hline & 2.82590500 & 4.08529700 & -3.10579100 \\
\hline & 2.71825600 & 5.72115300 & -2.45098500 \\
\hline & 1.37218700 & 3.16177800 & -1.481111 \\
\hline
\end{tabular}




\begin{tabular}{|c|c|c|c|}
\hline $\mathrm{H}$ & 0.69196100 & 4.77951500 & -1.65196300 \\
\hline C & 6.78013300 & 3.76240700 & 1.11888800 \\
\hline $\mathrm{H}$ & 7.62872500 & 3.17955300 & 1.51206700 \\
\hline $\mathrm{H}$ & 7.16371500 & 4.51935600 & 0.41577300 \\
\hline $\mathrm{H}$ & 6.28821100 & 4.29146600 & 1.95059200 \\
\hline 0 & 5.27765600 & 1.25046300 & 1.23757800 \\
\hline 0 & 6.46815100 & 1.65021400 & -0.88703500 \\
\hline C & 5.21460100 & 1.16703100 & 2.66480200 \\
\hline $\mathrm{H}$ & 4.34995200 & 0.54258700 & 2.92784100 \\
\hline $\mathrm{H}$ & 6.14888100 & 0.71007000 & 3.02472000 \\
\hline $\mathrm{H}$ & 5.08368800 & 2.16574300 & 3.11089000 \\
\hline C & 6.62156800 & 1.87137900 & -2.28442700 \\
\hline $\mathrm{H}$ & 5.65426200 & 1.76928400 & -2.80213200 \\
\hline $\mathrm{H}$ & 7.05296200 & 2.86663800 & -2.48166000 \\
\hline $\mathrm{H}$ & 7.31011100 & 1.10595200 & -2.66971500 \\
\hline Si & 5.62562200 & 2.58490900 & 0.26252500 \\
\hline C & -3.63847700 & 3.21929400 & 1.66245000 \\
\hline $\mathrm{H}$ & -2.99799000 & 4.11889200 & 1.66340200 \\
\hline $\mathrm{H}$ & -3.21724100 & 2.54073600 & 2.43032300 \\
\hline C & -5.10836700 & 3.55013500 & 1.97787600 \\
\hline C & -5.28819300 & 3.83332200 & 3.47879100 \\
\hline $\mathrm{H}$ & -6.34224900 & 4.05217700 & 3.71874600 \\
\hline $\mathrm{H}$ & -4.68262000 & 4.70321900 & 3.78509700 \\
\hline $\mathrm{H}$ & -4.97165900 & 2.97175800 & 4.08777000 \\
\hline C & -2.32197700 & 3.26151900 & -2.03412500 \\
\hline $\mathrm{H}$ & -2.85024300 & 2.57086200 & -2.69878700 \\
\hline $\mathrm{H}$ & -1.24682600 & 3.11402800 & -1.90379600 \\
\hline C & -2.95143000 & 4.33486900 & -1.50423000 \\
\hline $\mathrm{H}$ & -4.01699700 & 4.47115700 & -1.72346100 \\
\hline C & -2.27733500 & 5.42147300 & -0.72418900 \\
\hline $\mathrm{H}$ & -2.91528500 & 5.78659700 & 0.09262700 \\
\hline $\mathrm{H}$ & -1.31185000 & 5.09329700 & -0.31336800 \\
\hline H & -2.09402600 & 6.27980500 & -1.39495700 \\
\hline $\mathrm{H}$ & -5.72542500 & 2.66181400 & 1.74287500 \\
\hline C & -5.63400500 & 4.71729100 & 1.13583400 \\
\hline $\mathrm{H}$ & -5.57003700 & 4.49779900 & 0.06027400 \\
\hline $\mathrm{H}$ & -6.68960600 & 4.93077100 & 1.36672900 \\
\hline $\mathrm{H}$ & -5.05633000 & 5.63535900 & 1.34035400 \\
\hline
\end{tabular}

$\mathrm{MgCl}_{2}$-Ti-isobutyl-CMDMS-Mg2-Propylene-1,2-si-insertion-TS

$\begin{array}{llll}\text { Cl } & 7.17227000 & -2.10118700 & -1.29953600 \\ \text { Mg } & 6.09028600 & -3.88205000 & 0.09181600 \\ \text { Cl } & 8.21350800 & -0.23246400 & 1.39764100 \\ \text { Mg } & 6.12522800 & -0.24208600 & 0.01322500\end{array}$




\begin{tabular}{|c|c|c|c|}
\hline $\mathrm{Cl}$ & 4.00191000 & -3.89167600 & -1.29243000 \\
\hline $\mathrm{Cl}$ & 0.86650400 & -2.04210400 & $-1.3638460 e$ \\
\hline $\mathrm{Cl}$ & 4.03676300 & -0.25167800 & -1.37085200 \\
\hline $\mathrm{Cl}$ & 1.87286900 & -3.81337100 & 1.41177200 \\
\hline Mg & -0.21537700 & -3.82301400 & $0.0276510 e$ \\
\hline $\mathrm{Cl}$ & 5.04321100 & -2.02286800 & 1.40473000 \\
\hline Mg & 2.95491700 & -2.03242600 & 0.02030600 \\
\hline $\mathrm{Cl}$ & 1.90774900 & -0.17332400 & 1.33330900 \\
\hline Mg & -0.18200200 & -0.18334600 & -0.05095400 \\
\hline $\mathrm{Cl}$ & -2.30383100 & -3.83259100 & $-1.3567310 e$ \\
\hline $\mathrm{Cl}$ & -5.43937500 & -1.98309000 & -1.42816700 \\
\hline $\mathrm{Cl}$ & -2.27358800 & -0.10625200 & -1.47504900 \\
\hline $\mathrm{Cl}$ & -4.43306100 & -3.75429100 & 1.34740700 \\
\hline Mg & -6.52133300 & -3.76393000 & -0.03678000 \\
\hline $\mathrm{Cl}$ & -1.26252800 & -1.96381400 & $1.3405510 e$ \\
\hline Mg & -3.35058100 & -1.97382900 & -0.04398906 \\
\hline $\mathrm{Cl}$ & -4.32399700 & -0.06574500 & $1.3287170 e$ \\
\hline Mg & -6.48553300 & -0.12362900 & -0.11519406 \\
\hline $\mathrm{Cl}$ & -8.57481000 & -0.13359400 & -1.49947200 \\
\hline $\mathrm{Cl}$ & -7.56840100 & -1.90477100 & 1.27611200 \\
\hline $\mathrm{Ti}$ & -3.26457900 & 1.94208300 & -0.06270800 \\
\hline $\mathrm{Cl}$ & -5.51800700 & 1.89865500 & -1.07915300 \\
\hline $\mathrm{Cl}$ & -1.03361900 & 1.88373900 & 0.95799700 \\
\hline C & 4.06899400 & 3.25516700 & -0.44940000 \\
\hline C & 3.02387300 & 3.51452500 & 0.66871300 \\
\hline C & 4.12583800 & 4.44733900 & -1.42068000 \\
\hline $\mathrm{H}$ & 3.73016500 & 2.37137600 & -1.01790900 \\
\hline C & 1.81283100 & 4.31972400 & 0.14125200 \\
\hline $\mathrm{H}$ & 3.47400200 & 4.06221600 & 1.51727200 \\
\hline $\mathrm{H}$ & 2.68768300 & 2.54368800 & 1.06383500 \\
\hline C & 2.78517300 & 4.57245500 & -2.18604100 \\
\hline $\mathrm{H}$ & 4.32227200 & 5.37043400 & -0.84711400 \\
\hline $\mathrm{H}$ & 4.95622300 & 4.35637900 & -2.14057400 \\
\hline C & 1.59094200 & 4.05736100 & -1.35164000 \\
\hline $\mathrm{H}$ & 1.97806300 & 5.40191000 & 0.28817100 \\
\hline $\mathrm{H}$ & 0.91854800 & 4.05600800 & 0.72626600 \\
\hline $\mathrm{H}$ & 2.84174300 & 4.00684200 & -3.13102300 \\
\hline $\mathrm{H}$ & 2.61939900 & 5.62684000 & -2.46556100 \\
\hline $\mathrm{H}$ & 1.46313200 & 2.97048600 & -1.50375400 \\
\hline $\mathrm{H}$ & 0.65790900 & 4.52545200 & -1.70258300 \\
\hline C & 6.84271100 & 3.77440000 & 1.10762200 \\
\hline $\mathrm{H}$ & 7.68170200 & 3.17719000 & 1.50033600 \\
\hline $\mathrm{H}$ & 7.23812500 & 4.52416000 & 0.40343300 \\
\hline $\mathrm{H}$ & 6.35919500 & 4.31128100 & 1.9393030 \\
\hline
\end{tabular}




$\begin{array}{lrrr}\text { O } & 5.29902800 & 1.28594400 & 1.23714100 \\ \text { O } & 6.48741800 & 1.66158300 & -0.89686400 \\ \text { C } & 5.23639800 & 1.20180100 & 2.66417600 \\ \text { H } & 4.37721100 & 0.56898000 & 2.92539900 \\ \text { H } & 6.17406300 & 0.75275500 & 3.02525800 \\ \text { H } & 5.09456100 & 2.19929500 & 3.10966400 \\ \text { C } & 6.61696100 & 1.87165400 & -2.29873700 \\ \text { H } & 5.63780700 & 1.78457300 & -2.79667200 \\ \text { H } & 7.06258400 & 2.85759000 & -2.51036700 \\ \text { H } & 7.28306500 & 1.09079900 & -2.69215100 \\ \text { Si } & 5.67028700 & 2.61221800 & 0.25708200 \\ \text { C } & -3.79296300 & 3.69772900 & 1.08411000 \\ \text { H } & -2.98671300 & 4.18448800 & 1.65267000 \\ \text { H } & -3.92159000 & 2.69195200 & 1.59450500 \\ \text { C } & -5.12668100 & 4.44092800 & 1.20203700 \\ \text { C } & -5.87901200 & 3.96352100 & 2.45771200 \\ \text { H } & -6.85113800 & 4.47392000 & 2.54922700 \\ \text { H } & -5.29922700 & 4.17010600 & 3.37332600 \\ \text { H } & -6.07298000 & 2.87923700 & 2.41797000 \\ \text { C } & -2.58195600 & 3.17312600 & -1.75992100 \\ \text { H } & -3.25661400 & 2.80041200 & -2.53621900 \\ \text { H } & -1.51761200 & 2.94449100 & -1.87910000 \\ \text { C } & -2.95394200 & 4.28548500 & -0.99274700 \\ \text { H } & -3.95038800 & 4.69682900 & -1.17497500 \\ \text { C } & -1.91256000 & 5.23393900 & -0.46109100 \\ \text { H } & -2.30112800 & 5.92303100 & 0.29804800 \\ \text { H } & -1.05938900 & 4.68536500 & -0.04252000 \\ \text { H } & -1.54267800 & 5.83860300 & -1.30699200 \\ \text { H } & -5.74694600 & 4.16486200 & 0.33412700 \\ \text { C } & -4.96210900 & 5.96528400 & 1.22100900 \\ \text { H } & -4.52361000 & 6.35365500 & 0.28971700 \\ \text { H } & -5.93999300 & 6.45575600 & 1.34714400 \\ & -4.31714500 & 6.28429700 & 2.05785600\end{array}$

$\mathrm{MgCl}_{2}$-Ti-isobutyl-CMDMS-Mg2-Propylene-2,1-re-coordination

$\begin{array}{lrrr}\text { Cl } & -6.90408700 & -2.47057700 & 1.41118100 \\ \text { Mg } & -5.76948500 & -4.14764500 & -0.06467900 \\ \text { Cl } & -8.09857300 & -0.56595200 & -1.19585100 \\ \text { Mg } & -5.98028600 & -0.51862300 & 0.14140100 \\ \text { Cl } & -3.65110900 & -4.10030600 & 1.27240300 \\ \text { Cl } & -0.60892700 & -2.10093900 & 1.33961100 \\ \text { Cl } & -3.86182400 & -0.47123700 & 1.47830600 \\ \text { Cl } & -1.59271200 & -3.82534100 & -1.47333200 \\ \text { Mg } & 0.52565300 & -3.77799900 & -0.13625200\end{array}$




\begin{tabular}{|c|c|c|c|}
\hline $\mathrm{Cl}$ & -4.84566000 & -2.19559600 & -1.33460900 \\
\hline Mg & -2.72731200 & -2.14828000 & 0.00255700 \\
\hline $\mathrm{Cl}$ & -1.80346300 & -0.19630100 & -1.26737200 \\
\hline Mg & 0.31527500 & -0.14911200 & 0.06980200 \\
\hline $\mathrm{Cl}$ & 2.64395400 & -3.73061500 & 1.20084600 \\
\hline $\mathrm{Cl}$ & 5.68624500 & -1.73126900 & 1.26812500 \\
\hline $\mathrm{Cl}$ & 2.47079100 & -0.07513400 & 1.44144100 \\
\hline $\mathrm{Cl}$ & 4.70254300 & -3.45568200 & -1.54483600 \\
\hline Mg & 6.82079800 & -3.40836700 & -0.20781800 \\
\hline $\mathrm{Cl}$ & 1.44948200 & -1.82595200 & -1.40622700 \\
\hline Mg & 3.56772500 & -1.77863300 & -0.06921200 \\
\hline $\mathrm{Cl}$ & 4.42321000 & 0.26744300 & -1.38138700 \\
\hline Mg & 6.60985500 & 0.22065800 & -0.00183900 \\
\hline $\mathrm{Cl}$ & 8.72845800 & 0.26802000 & 1.33520300 \\
\hline $\mathrm{Cl}$ & 7.74465800 & -1.45631700 & -1.47772200 \\
\hline $\mathrm{Ti}$ & 3.33076500 & 2.15299900 & 0.05003600 \\
\hline $\mathrm{Cl}$ & 5.50244000 & 2.13035500 & 1.13360700 \\
\hline $\mathrm{Cl}$ & 0.88297400 & 2.11236300 & -0.47994200 \\
\hline C & -4.02521400 & 3.16293600 & 0.31469400 \\
\hline C & -3.12611900 & 3.42906400 & -0.93035700 \\
\hline C & -4.15921200 & 4.43695000 & 1.16963500 \\
\hline $\mathrm{H}$ & -3.53798100 & 2.38016000 & 0.92409300 \\
\hline C & -2.03379400 & 4.48483100 & -0.64413000 \\
\hline $\mathrm{H}$ & -3.72979400 & 3.76788100 & -1.79275500 \\
\hline $\mathrm{H}$ & -2.66241500 & 2.47744400 & -1.23003100 \\
\hline C & -2.78706800 & 4.85710100 & 1.74986000 \\
\hline $\mathrm{H}$ & -4.56343500 & 5.24894900 & 0.53966000 \\
\hline $\mathrm{H}$ & -4.88360000 & 4.30409300 & 1.99074500 \\
\hline C & -1.62022700 & 4.44362600 & 0.82843100 \\
\hline $\mathrm{H}$ & -2.40525300 & 5.49697300 & -0.88374800 \\
\hline $\mathrm{H}$ & -1.16413300 & 4.30687800 & -1.29611100 \\
\hline $\mathrm{H}$ & -2.64716500 & 4.40093400 & 2.74465800 \\
\hline $\mathrm{H}$ & -2.77690100 & 5.94910700 & 1.90719800 \\
\hline $\mathrm{H}$ & -1.30321900 & 3.41397400 & 1.06074700 \\
\hline $\mathrm{H}$ & -0.74085700 & 5.08380100 & 1.01076300 \\
\hline C & -6.91528100 & 3.38996600 & -1.04472600 \\
\hline $\mathrm{H}$ & -7.72159200 & 2.71093400 & -1.36746100 \\
\hline $\mathrm{H}$ & -7.33180400 & 4.13101200 & -0.34356200 \\
\hline $\mathrm{H}$ & -6.52297400 & 3.93175000 & -1.91975700 \\
\hline 0 & -5.23030400 & 1.00098100 & -1.16654200 \\
\hline 0 & -6.31197500 & 1.40883300 & 1.01784800 \\
\hline C & -5.21002200 & 0.88936800 & -2.59298700 \\
\hline $\mathrm{H}$ & -4.32407600 & 0.30406500 & -2.87399100 \\
\hline $\mathrm{H}$ & -6.13087200 & 0.37864300 & -2.9122880 \\
\hline
\end{tabular}




$\begin{array}{lrrr}\text { H } & -5.14511600 & 1.88326200 & -3.06333400 \\ \text { C } & -6.35412200 & 1.68596600 & 2.41356600 \\ \text { H } & -5.34069800 & 1.66246400 & 2.84555300 \\ \text { H } & -6.82554400 & 2.66376100 & 2.60695400 \\ \text { H } & -6.95899400 & 0.90290000 & 2.89229100 \\ \text { Si } & -5.61601600 & 2.34675000 & -0.22469300 \\ \text { C } & 3.49673100 & 3.56616700 & -1.43339600 \\ \text { H } & 4.49363100 & 3.89474900 & -1.06146900 \\ \text { H } & 3.67820700 & 2.96049300 & -2.34165200 \\ \text { C } & 2.54417700 & 4.74373100 & -1.70472700 \\ \text { C } & 1.71001000 & 4.49695100 & -2.96735700 \\ \text { C } & 3.30323800 & 6.07579500 & -1.78716300 \\ \text { H } & 1.82987000 & 4.82557500 & -0.86521300 \\ \text { H } & 0.97119700 & 5.30109500 & -3.11710300 \\ \text { H } & 1.16516700 & 3.54366600 & -2.90246800 \\ \text { H } & 2.35772500 & 4.46146800 & -3.86013100 \\ \text { H } & 2.61250700 & 6.91773900 & -1.96045000 \\ \text { H } & 4.03250000 & 6.06015500 & -2.61456500 \\ \text { H } & 3.85835800 & 6.28121200 & -0.85660900 \\ \text { C } & 2.55019700 & 3.11475000 & 2.60192000 \\ \text { H } & 3.36026000 & 2.56345400 & 3.09654900 \\ \text { C } & 2.88626000 & 4.10486000 & 1.74505700 \\ \text { H } & 2.11442900 & 4.70185400 & 1.24992300 \\ \text { H } & 3.92652100 & 4.41497800 & 1.61585900 \\ \text { C } & 1.15548000 & 2.76053500 & 3.00782700 \\ \text { H } & 1.00627200 & 1.67093200 & 3.01950300 \\ \text { H } & 0.99007900 & 3.11133600 & 4.04196600 \\ \text { H } & 0.40567500 & 3.22538500 & 2.35568800\end{array}$

$\mathrm{MgCl}_{2}$-Ti-isobutyl-CMDMS-Mg2-Propylene-2,1-re-insertion-TS

$\begin{array}{lrrr}\text { Cl } & -6.90440000 & -2.42382700 & 1.50369800 \\ \text { Mg } & -5.79385600 & -4.13600500 & 0.05001600 \\ \text { Cl } & -8.11892900 & -0.56694200 & -1.12835800 \\ \text { Mg } & -5.98576100 & -0.50257300 & 0.18432000 \\ \text { Cl } & -3.66061900 & -4.07163000 & 1.36252900 \\ \text { Cl } & -0.60855500 & -2.08595200 & 1.35566000 \\ \text { Cl } & -3.85244000 & -0.43815200 & 1.49664100 \\ \text { Cl } & -1.63150200 & -3.86247500 & -1.41066700 \\ \text { Mg } & 0.50171700 & -3.79811400 & -0.09818800 \\ \text { Cl } & -4.87522100 & -2.21466200 & -1.26952300 \\ \text { Mg } & -2.74201300 & -2.15030100 & 0.04305600 \\ \text { Cl } & -1.82338000 & -0.22898500 & -1.27646400 \\ \text { Mg } & 0.30989200 & -0.16491300 & 0.03595200 \\ \text { Cl } & 2.63489900 & -3.73368200 & 1.21437100\end{array}$




\begin{tabular}{|c|c|c|c|}
\hline $\mathrm{Cl}$ & 5.68681200 & -1.74799900 & 1.20746400 \\
\hline $\mathrm{Cl}$ & 2.45907500 & -0.12343300 & 1.47211300 \\
\hline $\mathrm{Cl}$ & 4.66420900 & -3.52453400 & -1.55878100 \\
\hline Mg & 6.79730300 & -3.46019500 & -0.24633500 \\
\hline $\mathrm{Cl}$ & 1.42037000 & -1.87670600 & -1.41780400 \\
\hline Mg & 3.55333000 & -1.81223800 & -0.10514400 \\
\hline $\mathrm{Cl}$ & 4.35016300 & 0.22990000 & -1.38398600 \\
\hline Mg & 6.60557900 & 0.17316500 & -0.11216600 \\
\hline $\mathrm{Cl}$ & 8.73871700 & 0.23763000 & 1.20037400 \\
\hline $\mathrm{Cl}$ & 7.71599100 & -1.53886800 & -1.56581300 \\
\hline $\mathrm{Ti}$ & 3.28584900 & 2.07996900 & 0.31094300 \\
\hline $\mathrm{Cl}$ & 5.62549900 & 2.17905900 & 0.95780500 \\
\hline $\mathrm{Cl}$ & 0.98876200 & 2.01789400 & -0.67850600 \\
\hline C & -4.00172500 & 3.17525100 & 0.16088400 \\
\hline C & -3.14934900 & 3.38671100 & -1.12695500 \\
\hline C & -4.08842200 & 4.48050000 & 0.97254700 \\
\hline $\mathrm{H}$ & -3.49959900 & 2.40829500 & 0.77836000 \\
\hline C & -2.03124800 & 4.43448800 & -0.92163500 \\
\hline $\mathrm{H}$ & -3.78290900 & 3.70619900 & -1.97498200 \\
\hline $\mathrm{H}$ & -2.71092400 & 2.41907200 & -1.41208000 \\
\hline C & -2.68976000 & 4.90459900 & 1.48330900 \\
\hline $\mathrm{H}$ & -4.50836800 & 5.27347200 & 0.32870100 \\
\hline $\mathrm{H}$ & -4.78141400 & 4.38677300 & 1.82535300 \\
\hline C & -1.56376600 & 4.44184100 & 0.53478500 \\
\hline $\mathrm{H}$ & -2.39498300 & 5.44298500 & -1.18732800 \\
\hline $\mathrm{H}$ & -1.18872600 & 4.21628200 & -1.59723300 \\
\hline $\mathrm{H}$ & -2.51612200 & 4.48403200 & 2.48823200 \\
\hline $\mathrm{H}$ & -2.66104000 & 6.00153600 & 1.59856700 \\
\hline $\mathrm{H}$ & -1.25053100 & 3.41761200 & 0.79343400 \\
\hline $\mathrm{H}$ & -0.66972400 & 5.07537200 & 0.66090800 \\
\hline C & -6.93643200 & 3.35517200 & -1.12177200 \\
\hline $\mathrm{H}$ & -7.74547800 & 2.65721300 & -1.39415400 \\
\hline $\mathrm{H}$ & -7.33939200 & 4.12107500 & -0.43968200 \\
\hline $\mathrm{H}$ & -6.57284800 & 3.86320300 & -2.02880200 \\
\hline 0 & -5.24825900 & 0.96680100 & -1.19376500 \\
\hline 0 & -6.27778600 & 1.45959100 & 0.99785200 \\
\hline C & -5.24925300 & 0.80873900 & -2.61597300 \\
\hline $\mathrm{H}$ & -4.36335600 & 0.22120800 & -2.89263400 \\
\hline $\mathrm{H}$ & -6.17134400 & 0.28216500 & -2.90418800 \\
\hline $\mathrm{H}$ & -5.20034000 & 1.78705000 & -3.11979500 \\
\hline C & -6.29258300 & 1.79481900 & 2.38126800 \\
\hline $\mathrm{H}$ & -5.27156400 & 1.78431900 & 2.79536300 \\
\hline $\mathrm{H}$ & -6.75632700 & 2.78216300 & 2.54202300 \\
\hline $\mathrm{H}$ & -6.89169900 & 1.03577500 & 2.90384300 \\
\hline
\end{tabular}




$\begin{array}{lrrr}\text { Si } & -5.61253800 & 2.34795300 & -0.29652200 \\ \text { C } & 3.46838000 & 4.03070200 & -0.60979800 \\ \text { H } & 4.43873000 & 4.53168500 & -0.48334100 \\ \text { H } & 3.70137400 & 3.15121800 & -1.29564500 \\ \text { C } & 2.42513200 & 4.93084100 & -1.28180200 \\ \text { C } & 2.32250900 & 4.62883000 & -2.78573300 \\ \text { C } & 2.73101000 & 6.41452200 & -1.04013000 \\ \text { H } & 1.43988800 & 4.70061200 & -0.84245800 \\ \text { H } & 1.56621500 & 5.27453500 & -3.26051200 \\ \text { H } & 2.02727900 & 3.58244000 & -2.95800300 \\ \text { H } & 3.28540700 & 4.80749100 & -3.29352500 \\ \text { H } & 1.93989700 & 7.05083300 & -1.46723300 \\ \text { H } & 3.68524500 & 6.69820000 & -1.51539100 \\ \text { H } & 2.81051300 & 6.65536000 & 0.03178300 \\ \text { C } & 2.63416500 & 2.99863700 & 2.26055900 \\ \text { H } & 3.39852900 & 2.48201300 & 2.85852300 \\ \text { C } & 3.04696500 & 4.16131000 & 1.60538400 \\ \text { H } & 2.29744900 & 4.90829700 & 1.33816000 \\ \text { H } & 4.06332300 & 4.53117500 & 1.74794800 \\ \text { C } & 1.19632300 & 2.78315300 & 2.68025000 \\ \text { H } & 0.95617700 & 1.71716200 & 2.79837900 \\ \text { H } & 1.01718800 & 3.26329300 & 3.65821900 \\ \text { H } & 0.49804800 & 3.21866600 & 1.95497100\end{array}$

$\mathrm{MgCl}_{2}$-Ti-isobutyl-CMDMS-Mg2-Propylene-2,1-si-coordination

$\begin{array}{lrrr}\text { Cl } & -6.98356200 & -2.37894400 & 1.36539500 \\ \text { Mg } & -5.85702100 & -4.06699800 & -0.10410200 \\ \text { Cl } & -8.14128300 & -0.46061600 & -1.24816700 \\ \text { Mg } & -6.03128400 & -0.43608100 & 0.10273100 \\ \text { Cl } & -3.74693200 & -4.04245300 & 1.24662600 \\ \text { Cl } & -0.68455800 & -2.07497100 & 1.33459700 \\ \text { Cl } & -3.92110700 & -0.41149000 & 1.45328300 \\ \text { Cl } & -1.66811700 & -3.78756400 & -1.48563000 \\ \text { Mg } & 0.44196100 & -3.76301600 & -0.13490400 \\ \text { Cl } & -4.90471700 & -2.12403900 & -1.36691600 \\ \text { Mg } & -2.79465700 & -2.09951700 & -0.01610400 \\ \text { Cl } & -1.84232900 & -0.15662900 & -1.27891700 \\ \text { Mg } & 0.26812000 & -0.13223900 & 0.07190600 \\ \text { Cl } & 2.55197500 & -3.73842600 & 1.21584100 \\ \text { Cl } & 5.61445700 & -1.77096600 & 1.30388300 \\ \text { Cl } & 2.40593800 & -0.04445900 & 1.43576600 \\ \text { Cl } & 4.63098100 & -3.48357100 & -1.51636200 \\ \text { Mg } & 6.74094900 & -3.45904900 & -0.16569800 \\ \text { Cl } & 1.39426900 & -1.82006000 & -1.39776300\end{array}$




\begin{tabular}{|c|c|c|c|}
\hline Mg & 3.50422500 & -1.79553400 & -0.04710200 \\
\hline $\mathrm{Cl}$ & 4.40550600 & 0.20602600 & -1.37617600 \\
\hline Mg & 6.56654500 & 0.17187300 & 0.04103300 \\
\hline $\mathrm{Cl}$ & 8.67686200 & 0.19643800 & 1.39172400 \\
\hline $\mathrm{Cl}$ & 7.69328800 & -1.51609000 & -1.42848600 \\
\hline $\mathrm{Ti}$ & 3.33037000 & 2.15696700 & -0.04100200 \\
\hline $\mathrm{Cl}$ & 5.46795100 & 2.13379700 & 1.09862000 \\
\hline $\mathrm{Cl}$ & 0.89888800 & 2.08654400 & -0.61313500 \\
\hline$C$ & -3.95560400 & 3.13561900 & 0.47131100 \\
\hline C & -3.01796400 & 3.48434700 & -0.72142500 \\
\hline C & -4.04120200 & 4.31874500 & 1.45403100 \\
\hline $\mathrm{H}$ & -3.51519000 & 2.27591900 & 1.00596500 \\
\hline C & -1.87620100 & 4.43862700 & -0.30013500 \\
\hline $\mathrm{H}$ & -3.58163400 & 3.95145200 & -1.55035700 \\
\hline $\mathrm{H}$ & -2.59863600 & 2.54722200 & -1.11783700 \\
\hline C & -2.66230600 & 4.58466300 & 2.10620900 \\
\hline $\mathrm{H}$ & -4.37902800 & 5.21734900 & 0.90797200 \\
\hline $\mathrm{H}$ & -4.79328600 & 4.14445200 & 2.24154500 \\
\hline C & -1.49347700 & 4.21594100 & 1.16580700 \\
\hline $\mathrm{H}$ & -2.18953800 & 5.48996200 & -0.42849100 \\
\hline $\mathrm{H}$ & -1.00554000 & 4.28802900 & -0.95705400 \\
\hline $\mathrm{H}$ & -2.57790800 & 4.00599300 & 3.04146700 \\
\hline $\mathrm{H}$ & -2.59465000 & 5.64785800 & 2.39322400 \\
\hline $\mathrm{H}$ & -1.22102400 & 3.15417300 & 1.29587400 \\
\hline $\mathrm{H}$ & -0.59238300 & 4.79421800 & 1.43045000 \\
\hline C & -6.78262900 & 3.58927900 & -0.95525800 \\
\hline $\mathrm{H}$ & -7.62803600 & 2.98874600 & -1.32822800 \\
\hline $\mathrm{H}$ & -7.16315800 & 4.32556400 & -0.22884500 \\
\hline $\mathrm{H}$ & -6.33289300 & 4.14327100 & -1.79439900 \\
\hline 0 & -5.23534100 & 1.10768700 & -1.14380400 \\
\hline 0 & -6.35466300 & 1.47759800 & 1.02280300 \\
\hline C & -5.20985600 & 1.03886800 & -2.57274400 \\
\hline $\mathrm{H}$ & -4.33846400 & 0.43818600 & -2.86701300 \\
\hline $\mathrm{H}$ & -6.14223300 & 0.56232400 & -2.91131700 \\
\hline $\mathrm{H}$ & -5.11561600 & 2.04459600 & -3.01210100 \\
\hline C & -6.46833500 & 1.69741500 & 2.42425400 \\
\hline $\mathrm{H}$ & -5.48810500 & 1.58433500 & 2.91460100 \\
\hline $\mathrm{H}$ & -6.88401300 & 2.69649700 & 2.63554300 \\
\hline $\mathrm{H}$ & -7.15424200 & 0.93859300 & 2.82712500 \\
\hline Si & -5.57029000 & 2.43240700 & -0.15193800 \\
\hline C & 3.58969400 & 3.50917000 & -1.57419800 \\
\hline $\mathrm{H}$ & 4.46956700 & 3.95744200 & -1.05763800 \\
\hline $\mathrm{H}$ & 3.98893400 & 2.89162000 & -2.40100600 \\
\hline C & 2.60889200 & 4.57313600 & -2.08977000 \\
\hline
\end{tabular}




$\begin{array}{llll}\text { C } & 1.87211000 & 4.07863000 & -3.34003400 \\ \text { C } & 3.31967500 & 5.90869400 & -2.35728000 \\ \text { H } & 1.84123500 & 4.75924900 & -1.31510800 \\ \text { H } & 1.11342800 & 4.80833200 & -3.66626100 \\ \text { H } & 1.36417000 & 3.12161500 & -3.15299200 \\ \text { H } & 2.58078900 & 3.93349800 & -4.17348200 \\ \text { H } & 2.60976200 & 6.67179100 & -2.71763800 \\ \text { H } & 4.10388700 & 5.78750900 & -3.12355200 \\ \text { H } & 3.80087400 & 6.29866700 & -1.44498300 \\ \text { C } & 2.33108800 & 3.21659800 & 2.37602000 \\ \text { C } & 2.80185600 & 4.13832200 & 1.50619700 \\ \text { H } & 2.15512200 & 4.53813700 & 0.71966200 \\ \text { H } & 3.78590500 & 4.59834900 & 1.63856400 \\ \text { H } & 1.31311100 & 2.84003700 & 2.21756200 \\ \text { C } & 3.05876800 & 2.70664800 & 3.57750300 \\ \text { H } & 2.52370300 & 3.03581800 & 4.48579700 \\ \text { H } & 3.06545700 & 1.60627100 & 3.59378200 \\ \text { H } & 4.09480800 & 3.06925700 & 3.61918200\end{array}$

$\mathrm{MgCl}_{2}$-Ti-isobutyl-CMDMS-Mg2-Propylene-2,1-si-insertion-TS

$\begin{array}{lrrr}\text { Cl } & 7.03686900 & -2.27798400 & -1.39753300 \\ \text { Mg } & 5.93873000 & -4.00221200 & 0.05131200 \\ \text { Cl } & 8.17671600 & -0.38110400 & 1.23946300 \\ \text { Mg } & 6.06208100 & -0.36665100 & -0.10429400 \\ \text { Cl } & 3.82398700 & -3.98775600 & -1.29227100 \\ \text { Cl } & 0.73485400 & -2.06183700 & -1.34230100 \\ \text { Cl } & 3.94724700 & -0.35215700 & -1.44770700 \\ \text { Cl } & 1.75101200 & -3.80058000 & 1.44988200 \\ \text { Mg } & -0.36372700 & -3.78612400 & 0.10630000 \\ \text { Cl } & 4.96389000 & -2.09078800 & 1.34469500 \\ \text { Mg } & 2.84919300 & -2.07633300 & 0.00098900 \\ \text { Cl } & 1.87429400 & -0.16504000 & 1.29441200 \\ \text { Mg } & -0.24156000 & -0.15090700 & -0.04974500 \\ \text { Cl } & -2.47837600 & -3.77159400 & -1.23732700 \\ \text { Cl } & -5.56801600 & -1.84570800 & -1.28769300 \\ \text { Cl } & -2.35670600 & -0.08764300 & -1.45787400 \\ \text { Cl } & -4.55156500 & -3.58450200 & 1.50476600 \\ \text { Mg } & -6.66619600 & -3.57005500 & 0.16125400 \\ \text { Cl } & -1.33853900 & -1.87478400 & 1.39970000 \\ \text { Mg } & -3.45316700 & -1.86014700 & 0.05656500 \\ \text { Cl } & -4.32609400 & 0.12368700 & 1.37416000 \\ \text { Mg } & -6.54238100 & 0.06554000 & 0.00588600 \\ \text { Cl } & -8.65764500 & 0.08001000 & -1.33777000 \\ \text { Cl } & -7.64105100 & -1.65863600 & 1.45462200\end{array}$




\begin{tabular}{|c|c|c|c|}
\hline $\mathrm{Ti}$ & -3.27054900 & 2.06358200 & -0.13389400 \\
\hline $\mathrm{Cl}$ & -5.58924600 & 2.12017600 & $-0.9432180 e$ \\
\hline $\mathrm{Cl}$ & -1.03723700 & 1.93664000 & 0.90007100 \\
\hline C & 3.88866500 & 3.12950600 & -0.39420800 \\
\hline C & 2.96195800 & 3.48777800 & 0.80208700 \\
\hline C & 3.89641600 & 4.27146100 & -1.42736100 \\
\hline $\mathrm{H}$ & 3.47322900 & 2.23123300 & -0.88310100 \\
\hline C & 1.74564400 & 4.33396300 & 0.35411400 \\
\hline $\mathrm{H}$ & 3.51415800 & 4.04426800 & 1.58181800 \\
\hline $\mathrm{H}$ & 2.61673300 & 2.55159200 & 1.26625600 \\
\hline C & 2.49392800 & 4.42727100 & -2.06589500 \\
\hline $\mathrm{H}$ & 4.19014800 & 5.21068100 & -0.92585200 \\
\hline $\mathrm{H}$ & 4.64429600 & 4.10592400 & -2.22010200 \\
\hline C & 1.36520000 & 4.01720800 & -1.09464900 \\
\hline $\mathrm{H}$ & 1.98053300 & 5.41056300 & 0.42962900 \\
\hline $\mathrm{H}$ & 0.89522900 & 4.14895000 & 1.02866700 \\
\hline $\mathrm{H}$ & 2.43227100 & 3.81281100 & -2.97957100 \\
\hline $\mathrm{H}$ & 2.35180200 & 5.47356900 & -2.38585700 \\
\hline $\mathrm{H}$ & 1.17000600 & 2.93317100 & -1.17512500 \\
\hline $\mathrm{H}$ & 0.42311200 & 4.51588600 & -1.37543700 \\
\hline C & 6.71518300 & 3.67646900 & 1.01626500 \\
\hline $\mathrm{H}$ & 7.58578200 & 3.10307800 & 1.37333900 \\
\hline $\mathrm{H}$ & 7.06148900 & 4.43730500 & 0.29799000 \\
\hline $\mathrm{H}$ & 6.25312500 & 4.20094800 & 1.86759600 \\
\hline 0 & 5.24875700 & 1.14033500 & 1.18099500 \\
\hline 0 & 6.34280500 & 1.57413600 & -0.98464600 \\
\hline C & 5.23984800 & 1.04715700 & 2.60869500 \\
\hline $\mathrm{H}$ & 4.38594400 & 0.42130200 & 2.90191200 \\
\hline $\mathrm{H}$ & 6.18630400 & 0.58647600 & 2.92966800 \\
\hline $\mathrm{H}$ & 5.12702500 & 2.04307700 & 3.06590100 \\
\hline C & 6.46686500 & 1.81842000 & -2.38105800 \\
\hline $\mathrm{H}$ & 5.49735100 & 1.67859800 & -2.88551200 \\
\hline $\mathrm{H}$ & 6.85043200 & 2.83406900 & -2.57294200 \\
\hline $\mathrm{H}$ & 7.18391900 & 1.08966000 & -2.78530500 \\
\hline Si & 5.53714600 & 2.48882100 & 0.20680000 \\
\hline $\mathrm{C}$ & -3.64473100 & 3.93385500 & 0.86925600 \\
\hline $\mathrm{H}$ & -4.59570900 & 4.42656500 & 0.61784500 \\
\hline $\mathrm{H}$ & -3.94446600 & 2.99225000 & 1.43060200 \\
\hline C & -2.76330000 & 4.78596000 & 1.78397300 \\
\hline C & -3.03871900 & 4.44750200 & 3.25913300 \\
\hline C & -2.95505000 & 6.28459000 & 1.52025400 \\
\hline $\mathrm{H}$ & -1.70822700 & 4.52520300 & 1.59040200 \\
\hline $\mathrm{H}$ & -2.40882800 & 5.06073300 & 3.92355800 \\
\hline $\mathrm{H}$ & -2.81823400 & 3.38872300 & 3.46884000 \\
\hline
\end{tabular}




$\begin{array}{llll}\text { H } & -4.09282400 & 4.63949500 & 3.52156400 \\ \text { H } & -2.28462500 & 6.88233400 & 2.15766600 \\ \text { H } & -3.99166900 & 6.58962500 & 1.74311500 \\ \text { H } & -2.74641000 & 6.55589700 & 0.47386000 \\ \text { C } & -2.46605900 & 3.12606200 & -1.96672600 \\ \text { C } & -2.67605300 & 4.24294000 & -1.16030900 \\ \text { H } & -1.83810800 & 4.66926100 & -0.60790500 \\ \text { H } & -3.50011300 & 4.92296200 & -1.38836600 \\ \text { H } & -1.45576500 & 2.69983100 & -1.93594800 \\ \text { C } & -3.27855600 & 2.82387000 & -3.20711400 \\ \text { H } & -2.73812100 & 3.19791400 & -4.09453400 \\ \text { H } & -3.43277100 & 1.74462900 & -3.34730200 \\ \text { H } & -4.26531200 & 3.30511100 & -3.17293000\end{array}$

\begin{tabular}{|c|c|c|c|}
\hline \multicolumn{4}{|c|}{$\mathrm{MgCl}_{2}$-Ti-isobutyl-DCPDMS-Mg2-Propylene-1,2-re-coordination } \\
\hline $\mathrm{Cl}$ & 6.36541200 & -3.06670100 & -1.37689500 \\
\hline Mg & 5.11582300 & -4.64656800 & 0.11357000 \\
\hline $\mathrm{Cl}$ & 7.67203400 & -1.21276900 & 1.21282500 \\
\hline Mg & 5.56722900 & -1.04285300 & -0.13567000 \\
\hline $\mathrm{Cl}$ & 3.01101900 & -4.47598900 & -1.23484100 \\
\hline $\mathrm{Cl}$ & 0.10815500 & -2.28082600 & -1.34205100 \\
\hline $\mathrm{Cl}$ & 3.46231400 & -0.87143900 & -1.48383600 \\
\hline $\mathrm{Cl}$ & 0.96351100 & -4.03116200 & 1.49673400 \\
\hline Mg & -1.14128200 & -3.86057900 & 0.14832500 \\
\hline $\mathrm{Cl}$ & 4.31759000 & -2.62165800 & 1.35500900 \\
\hline Mg & 2.21303500 & -2.45156900 & 0.00622300 \\
\hline $\mathrm{Cl}$ & 1.41487200 & -0.42685600 & 1.24760200 \\
\hline Mg & -0.69027200 & -0.25635800 & -0.10097600 \\
\hline $\mathrm{Cl}$ & -3.24602900 & -3.68996400 & -1.20010700 \\
\hline $\mathrm{Cl}$ & -6.14897300 & -1.49480400 & -1.30739100 \\
\hline $\mathrm{Cl}$ & -2.79839900 & -0.01724400 & -1.47298400 \\
\hline $\mathrm{Cl}$ & -5.29372500 & -3.24515800 & 1.53140800 \\
\hline Mg & -7.39843300 & -3.07460000 & 0.18305100 \\
\hline $\mathrm{Cl}$ & -1.93938700 & -1.83597400 & 1.38961700 \\
\hline Mg & -4.04410000 & -1.66545900 & 0.04131000 \\
\hline $\mathrm{Cl}$ & -4.79889100 & 0.42665500 & 1.34413800 \\
\hline Mg & -6.94680400 & 0.52965400 & -0.06606900 \\
\hline $\mathrm{Cl}$ & -9.05188300 & 0.70029600 & -1.41448800 \\
\hline $\mathrm{Cl}$ & -8.19650700 & -1.05000700 & 1.42429000 \\
\hline $\mathrm{Ti}$ & -3.58159500 & 2.25075800 & -0.03607100 \\
\hline $\mathrm{Cl}$ & -5.68016100 & 2.32791100 & -1.22339300 \\
\hline $\mathrm{Cl}$ & -1.23941300 & 1.94231700 & 0.74896600 \\
\hline C & 4.17423800 & 2.99153000 & -0.81539900 \\
\hline
\end{tabular}




\begin{tabular}{|c|c|c|c|}
\hline C & 2.75268800 & 2.37969900 & -0.97376500 \\
\hline C & 3.91714900 & 4.31227600 & -0.04618700 \\
\hline $\mathrm{H}$ & 4.54881300 & 3.27640300 & -1.81787300 \\
\hline C & 1.75654400 & 3.57083400 & -0.84971900 \\
\hline $\mathrm{H}$ & 2.56109800 & 1.63106300 & -0.19332700 \\
\hline $\mathrm{H}$ & 2.65909600 & 1.84184800 & -1.92679800 \\
\hline C & 2.62377100 & 4.83472200 & -0.68319200 \\
\hline $\mathrm{H}$ & 4.75481400 & 5.02408800 & -0.11121000 \\
\hline $\mathrm{H}$ & 3.74471400 & 4.10815100 & 1.02718700 \\
\hline $\mathrm{H}$ & 1.08395200 & 3.64702600 & -1.72007200 \\
\hline $\mathrm{H}$ & 1.11183900 & 3.43062600 & 0.03137900 \\
\hline $\mathrm{H}$ & 2.86242800 & 5.27009300 & -1.66932900 \\
\hline $\mathrm{H}$ & 2.13410800 & 5.62286200 & -0.08883100 \\
\hline C & 6.86882900 & 2.99378800 & 0.67605700 \\
\hline C & 8.03559900 & 2.25656400 & 1.38655400 \\
\hline C & 7.54409900 & 3.82128100 & -0.48063800 \\
\hline $\mathrm{H}$ & 6.35701500 & 3.67410400 & 1.37786000 \\
\hline C & 9.08284800 & 2.08050600 & 0.28312500 \\
\hline $\mathrm{H}$ & 7.75721800 & 1.29299800 & 1.83442500 \\
\hline $\mathrm{H}$ & 8.43961500 & 2.90355400 & 2.18547000 \\
\hline C & 9.04079500 & 3.42347600 & -0.45874400 \\
\hline $\mathrm{H}$ & 7.40353500 & 4.90410400 & -0.33981600 \\
\hline $\mathrm{H}$ & 7.10479800 & 3.59507900 & -1.46809400 \\
\hline $\mathrm{H}$ & 10.08057100 & 1.83028100 & 0.67539600 \\
\hline $\mathrm{H}$ & 8.77640400 & 1.24794800 & -0.37166100 \\
\hline $\mathrm{H}$ & 9.61325300 & 4.17520500 & 0.11100300 \\
\hline $\mathrm{H}$ & 9.48073600 & 3.38279200 & -1.46809900 \\
\hline 0 & 4.95631900 & 0.71407900 & 0.98610400 \\
\hline 0 & 6.12457300 & 0.76771600 & -1.16474700 \\
\hline C & 6.23223000 & 0.85772700 & -2.58186000 \\
\hline $\mathrm{H}$ & 5.23563700 & 0.94243900 & -3.04329700 \\
\hline $\mathrm{H}$ & 6.86135900 & 1.71310600 & -2.87560500 \\
\hline $\mathrm{H}$ & 6.70592900 & -0.06759200 & -2.93940100 \\
\hline C & 4.85509100 & 0.72275700 & 2.41274500 \\
\hline $\mathrm{H}$ & 3.84986300 & 0.37329700 & 2.68747300 \\
\hline $\mathrm{H}$ & 5.62486300 & 0.04614900 & 2.81365600 \\
\hline $\mathrm{H}$ & 5.01343900 & 1.73631800 & 2.81479700 \\
\hline Si & 5.52235000 & 1.92010200 & -0.05704100 \\
\hline C & -3.78332000 & 3.70776200 & 1.41281900 \\
\hline $\mathrm{H}$ & -4.67054700 & 4.17645400 & 0.93411000 \\
\hline $\mathrm{H}$ & -4.16357300 & 3.12174200 & 2.27146500 \\
\hline C & -2.72772100 & 4.73733600 & 1.85532400 \\
\hline C & -2.13170900 & 4.36017800 & 3.21663200 \\
\hline C & -3.29922500 & 6.16232400 & 1.87089400 \\
\hline
\end{tabular}




$\begin{array}{llll}\text { H } & -1.89080300 & 4.72535700 & 1.13209600 \\ \text { H } & -1.32605400 & 5.05536300 & 3.50367900 \\ \text { H } & -1.71166500 & 3.34363500 & 3.19598300 \\ \text { H } & -2.90585800 & 4.39608500 & 4.00228100 \\ \text { H } & -2.53280700 & 6.89702300 & 2.16876800 \\ \text { H } & -4.13781300 & 6.23977600 & 2.58318800 \\ \text { H } & -3.67908700 & 6.45653600 & 0.87806800 \\ \text { C } & -2.45798900 & 3.26598800 & -2.16270800 \\ \text { H } & -3.00662700 & 2.73292700 & -2.94618400 \\ \text { H } & -1.47333800 & 2.88362300 & -1.88484400 \\ \text { C } & -2.92012900 & 4.42658300 & -1.64504500 \\ \text { H } & -2.30252000 & 4.92920700 & -0.89072300 \\ \text { C } & -4.14986500 & 5.15962300 & -2.07907300 \\ \text { H } & -4.78807700 & 5.41834800 & -1.21976500 \\ \text { H } & -3.84613400 & 6.11303000 & -2.54613400 \\ \text { H } & -4.74725300 & 4.58405800 & -2.79828200\end{array}$

$\mathrm{MgCl}_{2}$-Ti-isobuty1-DCPDMS-Mg2-Propylene-1, 2-re-insertion-TS

\begin{tabular}{|c|c|c|c|}
\hline $\mathrm{Cl}$ & 6.37438800 & -3.04652000 & -1.40746800 \\
\hline Mg & 5.13366500 & -4.64606300 & $0.0693550 e$ \\
\hline $\mathrm{Cl}$ & 7.68307900 & -1.21689400 & 1.19844 \\
\hline Mg & 5.57392300 & -1.03855800 & -0.1421450 \\
\hline $\mathrm{Cl}$ & 3.02450900 & -4.46706600 & -1.2711410 \\
\hline $\mathrm{Cl}$ & 0.11503100 & -2.27936000 & $-1.346332 e$ \\
\hline $\mathrm{Cl}$ & 3.46465400 & -0.85872900 & $-1.482386 e$ \\
\hline $\mathrm{Cl}$ & 0.98358300 & -4.05780000 & 1.4708996 \\
\hline Mg & -1.12552000 & -3.87880600 & 0.1304450 \\
\hline $\mathrm{Cl}$ & 4.33314800 & -2.63704200 & 1.3349050 \\
\hline $\mathrm{Mg}$ & 2.22426500 & -2.45850200 & -0.0060060 \\
\hline $\mathrm{Cl}$ & 1.42380100 & -0.44967600 & $1.259507 €$ \\
\hline $\mathrm{Mg}$ & -0.68613800 & -0.27090200 & -0.081110 \\
\hline $\mathrm{Cl}$ & -3.23466100 & -3.69976500 & -1.2101110 \\
\hline $\mathrm{Cl}$ & -6.14424600 & -1.51206500 & -1.2853850 \\
\hline $\mathrm{Cl}$ & -2.79006800 & -0.04701400 & $-1.488377 €$ \\
\hline $\mathrm{Cl}$ & -5.27578200 & -3.29051100 & 1.53187400 \\
\hline Mg & -7.38483700 & -3.11154300 & $0.1914280 €$ \\
\hline $\mathrm{Cl}$ & -1.92593000 & -1.87006800 & 1.39583200 \\
\hline Mg & -4.03490900 & -1.69119900 & 0.0553320 \\
\hline $\mathrm{Cl}$ & -4.73521400 & 0.38711800 & $1.349036 e$ \\
\hline Mg & -6.94411100 & 0.49658400 & $-0.019911 €$ \\
\hline $\mathrm{Cl}$ & -9.05379000 & 0.67555200 & -1.360446 \\
\hline $\mathrm{Cl}$ & -8.18520900 & -1.10284300 & 1.4567536 \\
\hline $\mathrm{Ti}$ & -3.50202300 & 2.18806200 & -0.19397200 \\
\hline & .79065600 & .40568300 & -1.05366 \\
\hline
\end{tabular}




\begin{tabular}{|c|c|c|c|}
\hline $\mathrm{Cl}$ & -1.29848900 & 1.88228700 & 0.86190600 \\
\hline C & 4.13469000 & 2.99132400 & -0.75701200 \\
\hline c & 2.71470700 & 2.36553600 & -0.89315400 \\
\hline C & 3.88541200 & 4. 30748000 & 0.02383700 \\
\hline $\mathrm{H}$ & 4.48909900 & 3.28287400 & -1.76439500 \\
\hline C & 1.70707600 & 3.53203200 & -0.67308200 \\
\hline $\mathrm{H}$ & 2.55943600 & 1.57412000 & -0.14892400 \\
\hline $\mathrm{H}$ & 2.59499600 & 1.87884500 & -1.87074400 \\
\hline C & 2.55947800 & 4.80960100 & -0.56015500 \\
\hline $\mathrm{H}$ & 4.70957500 & 5.03147100 & -0.07200500 \\
\hline $\mathrm{H}$ & 3.75699700 & 4.10156400 & 1.10302700 \\
\hline $\mathrm{H}$ & 0.95884500 & 3.60126100 & -1.48007500 \\
\hline $\mathrm{H}$ & 1.14477000 & 3.36849300 & 0.25952200 \\
\hline $\mathrm{H}$ & 2.74731300 & 5.23700800 & -1.56088300 \\
\hline $\mathrm{H}$ & 2.08627400 & 5.59729200 & 0.04826100 \\
\hline C & 6.84509600 & 2.99951100 & 0.71238500 \\
\hline C & 8.01987200 & 2.25881900 & 1.40638500 \\
\hline C & 7.50902000 & 3.84231900 & -0.43975200 \\
\hline $\mathrm{H}$ & 6.33541600 & 3.66977200 & 1.42516000 \\
\hline C & 9.06045600 & 2.09942500 & 0.29418700 \\
\hline $\mathrm{H}$ & 7.74855700 & 1.28890700 & 1.84496000 \\
\hline $\mathrm{H}$ & 8.42654400 & 2.89802200 & 2.21017600 \\
\hline C & 9.00735300 & 3.44980800 & -0.43345700 \\
\hline H & 7.36584000 & 4.92294900 & -0.28536700 \\
\hline $\mathrm{H}$ & 7.06305400 & 3.62606400 & -1.42649300 \\
\hline $\mathrm{H}$ & 10.06188500 & 1.84993700 & 0.67732300 \\
\hline $\mathrm{H}$ & 8.75388900 & 1.27223000 & -0.36720300 \\
\hline $\mathrm{H}$ & 9.58134000 & 4.19795400 & 0.13943200 \\
\hline $\mathrm{H}$ & 9.43965600 & 3.42102600 & -1.44650100 \\
\hline 0 & 4.95548100 & 0.69910300 & 1.00620900 \\
\hline 0 & 6.11126400 & 0.79259900 & -1.14928400 \\
\hline C & 6.21567300 & 0.90097400 & -2.56538500 \\
\hline $\mathrm{H}$ & 5.21782500 & 0.98538800 & -3.02413400 \\
\hline $\mathrm{H}$ & 6.83930100 & 1.76359100 & -2.84951400 \\
\hline $\mathrm{H}$ & 6.69408700 & -0.01701800 & -2.93529100 \\
\hline C & 4.86091100 & 0.68902800 & 2.43317300 \\
\hline $\mathrm{H}$ & 3.85782600 & 0.33383600 & 2.70852300 \\
\hline $\mathrm{H}$ & 5.63412900 & 0.00890200 & 2.82119000 \\
\hline $\mathrm{H}$ & 5.01943800 & 1.69761200 & 2.84758100 \\
\hline Si & 5.50198600 & 1.92449200 & -0.02474400 \\
\hline C & -3.70232300 & 4.12106300 & 0.75942600 \\
\hline $\mathrm{H}$ & -4.61098100 & 4.70272400 & 0.54932300 \\
\hline $\mathrm{H}$ & -4.08467800 & 3.22651200 & 1.34550800 \\
\hline C & -2.69840500 & 4.87823600 & 1.6352510 \\
\hline
\end{tabular}




$\begin{array}{llll}\text { C } & -2.90460500 & 4.52189100 & 3.11738200 \\ \text { C } & -2.77447800 & 6.39488000 & 1.42058700 \\ \text { H } & -1.68272200 & 4.54060100 & 1.36654800 \\ \text { H } & -2.18153500 & 5.05778000 & 3.75314700 \\ \text { H } & -2.76406500 & 3.44228000 & 3.28458400 \\ \text { H } & -3.91884600 & 4.79530500 & 3.45459000 \\ \text { H } & -2.04355800 & 6.91520900 & 2.05940400 \\ \text { H } & -3.77724900 & 6.77760600 & 1.67624000 \\ \text { H } & -2.56221000 & 6.68212600 & 0.37932700 \\ \text { C } & -2.64506400 & 3.12106800 & -1.99637700 \\ \text { H } & -3.30394900 & 2.79117000 & -2.80708500 \\ \text { H } & -1.63634700 & 2.69885200 & -1.98068600 \\ \text { C } & -2.87158200 & 4.35760800 & -1.37779700 \\ \text { H } & -2.01670300 & 4.81132700 & -0.86998000 \\ \text { C } & -3.92519600 & 5.30035100 & -1.89511100 \\ \text { H } & -4.16935100 & 6.10259800 & -1.18745800 \\ \text { H } & -3.52855600 & 5.76996400 & -2.81208400 \\ \text { H } & -4.84575200 & 4.76221900 & -2.15959100\end{array}$

$\mathrm{MgCl}_{2}$-Ti-i sobutyl-DCPDMS-Mg2-Propylene-1, 2-si-coordination

$\begin{array}{lrrr}\text { Cl } & 6.50097500 & -2.94100400 & -1.28061000 \\ \text { Mg } & 5.25082600 & -4.54609100 & 0.18217000 \\ \text { Cl } & 7.73564500 & -1.07870500 & 1.33822100 \\ \text { Mg } & 5.65135700 & -0.93490000 & -0.04473100 \\ \text { Cl } & 3.16654500 & -4.40162900 & -1.20071100 \\ \text { Cl } & 0.23276900 & -2.25031700 & -1.34774100 \\ \text { Cl } & 3.56694500 & -0.78959800 & -1.42738500 \\ \text { Cl } & 1.06704700 & -3.99976700 & 1.49785800 \\ \text { Mg } & -1.01723100 & -3.85529900 & 0.11497100 \\ \text { Cl } & 4.40115700 & -2.53892800 & 1.41827800 \\ \text { Mg } & 2.31712100 & -2.39494000 & 0.03502100 \\ \text { Cl } & 1.46751800 & -0.38797700 & 1.27104800 \\ \text { Mg } & -0.61720100 & -0.24358600 & -0.11201000 \\ \text { Cl } & -3.10145700 & -3.71079600 & -1.26793900 \\ \text { Cl } & -6.03534500 & -1.55950000 & -1.41503500 \\ \text { Cl } & -2.71710300 & -0.04797100 & -1.51513700 \\ \text { Cl } & -5.20115300 & -3.30895500 & 1.43057200 \\ \text { Mg } & -7.28533200 & -3.16452400 & 0.04773900 \\ \text { Cl } & -1.86677800 & -1.84842100 & 1.35093600 \\ \text { Mg } & -3.95097300 & -1.70409300 & -0.03187900 \\ \text { Cl } & -4.77002300 & 0.35474300 & 1.27060000 \\ \text { Mg } & -6.88452900 & 0.44724600 & -0.17904600 \\ \text { Cl } & -8.96917500 & 0.59173100 & -1.56194600 \\ \text { Cl } & -8.13486600 & -1.15767100 & 1.28362400\end{array}$




\begin{tabular}{|c|c|c|c|}
\hline $\mathrm{Ti}$ & -3.55128100 & 2.19823800 & -0.05923300 \\
\hline $\mathrm{Cl}$ & -5.62946700 & 2.30936700 & -1.27104200 \\
\hline $\mathrm{Cl}$ & -1.15985600 & 1.98429300 & 0.65367500 \\
\hline C & 4.23501400 & 3.06797000 & -0.79546200 \\
\hline C & 2.80770500 & 2.45533000 & -0.91212100 \\
\hline C & 3.99479500 & 4.43428900 & -0.10386000 \\
\hline $\mathrm{H}$ & 4.60291300 & 3.29162100 & -1.81550100 \\
\hline C & 1.81867800 & 3.65331300 & -0.80538400 \\
\hline $\mathrm{H}$ & 2.62285200 & 1.72429000 & -0.11357200 \\
\hline $\mathrm{H}$ & 2.69865200 & 1.89660800 & -1.85120000 \\
\hline C & 2.69208400 & 4.92074200 & -0.74959900 \\
\hline $\mathrm{H}$ & 4.83385900 & 5.13677600 & -0.22643000 \\
\hline $\mathrm{H}$ & 3.83971000 & 4.29944800 & 0.98291700 \\
\hline $\mathrm{H}$ & 1.10201700 & 3.68101100 & -1.64233700 \\
\hline $\mathrm{H}$ & 1.22024800 & 3.56255200 & 0.11426800 \\
\hline $\mathrm{H}$ & 2.91122000 & 5.28267200 & -1.76952800 \\
\hline $\mathrm{H}$ & 2.21845800 & 5.75227600 & -0.20310300 \\
\hline C & 6.90625400 & 3.11803000 & 0.74561700 \\
\hline C & 8.06393300 & 2.40215400 & 1.48719600 \\
\hline C & 7.60128500 & 3.92559400 & -0.41195500 \\
\hline $\mathrm{H}$ & 6.37511800 & 3.80770100 & 1.42362200 \\
\hline C & 9.09398000 & 2.14372000 & 0.38405800 \\
\hline $\mathrm{H}$ & 7.77237600 & 1.47150300 & 1.99280400 \\
\hline $\mathrm{H}$ & 8.48651400 & 3.08699700 & 2.24443500 \\
\hline C & 9.07688200 & 3.44508600 & -0.43246700 \\
\hline $\mathrm{H}$ & 7.53067000 & 5.01053600 & -0.23848100 \\
\hline $\mathrm{H}$ & 7.12171100 & 3.75637200 & -1.39214900 \\
\hline $\mathrm{H}$ & 10.09027700 & 1.89263400 & 0.77915300 \\
\hline $\mathrm{H}$ & 8.76151200 & 1.28142800 & -0.21634800 \\
\hline $\mathrm{H}$ & 9.71477200 & 4.19812700 & 0.06012700 \\
\hline $\mathrm{H}$ & 9.46410900 & 3.32083600 & -1.45643700 \\
\hline 0 & 5.01011600 & 0.82095100 & 1.04874600 \\
\hline 0 & 6.21425700 & 0.86641500 & -1.08214000 \\
\hline C & 6.35979000 & 0.94679200 & -2.49602100 \\
\hline $\mathrm{H}$ & 5.37511400 & 1.01873000 & -2.98450400 \\
\hline $\mathrm{H}$ & 6.98860000 & 1.80620300 & -2.77866100 \\
\hline $\mathrm{H}$ & 6.85140900 & 0.02321200 & -2.83319900 \\
\hline C & 4.88322000 & 0.83960800 & 2.47343900 \\
\hline $\mathrm{H}$ & 3.87725300 & 0.48060900 & 2.73275100 \\
\hline $\mathrm{H}$ & 5.65337700 & 0.17490300 & 2.89311100 \\
\hline $\mathrm{H}$ & 5.02295300 & 1.85798300 & 2.87010900 \\
\hline Si & 5.58148700 & 2.02302600 & 0.00346500 \\
\hline C & -3.74112600 & 3.48421700 & 1.55832900 \\
\hline $\mathrm{H}$ & -2.98585500 & 4. 28964400 & 1.54850900 \\
\hline
\end{tabular}




$\begin{array}{llll}\text { H } & -3.42337300 & 2.77722500 & 2.35083300 \\ \text { C } & -5.15294800 & 4.02492000 & 1.84785200 \\ \text { C } & -5.28450500 & 4.41329900 & 3.33030000 \\ \text { H } & -6.29853200 & 4.78326800 & 3.55697600 \\ \text { H } & -4.56673100 & 5.21091300 & 3.58695800 \\ \text { H } & -5.08077000 & 3.55347100 & 3.98794800 \\ \text { C } & -2.47746700 & 3.22074100 & -2.18165600 \\ \text { H } & -3.15224500 & 2.62325800 & -2.80186600 \\ \text { H } & -1.43795400 & 2.89049400 & -2.10725400 \\ \text { C } & -2.88232900 & 4.38743600 & -1.63197500 \\ \text { H } & -3.92198300 & 4.69916700 & -1.78665400 \\ \text { C } & -1.98621700 & 5.34589600 & -0.91013400 \\ \text { H } & -2.48653500 & 5.78431900 & -0.03513500 \\ \text { H } & -1.04746800 & 4.86903900 & -0.59304600 \\ \text { H } & -1.74083000 & 6.18249500 & -1.58838400 \\ \text { H } & -5.88542400 & 3.21725800 & 1.65850400 \\ \text { C } & -5.51742500 & 5.20614000 & 0.94175200 \\ \text { H } & -5.51742600 & 4.91577700 & -0.11888100 \\ \text { H } & -6.52208300 & 5.59192600 & 1.17644000 \\ \text { H } & -4.80162200 & 6.03555500 & 1.07718700\end{array}$

$\mathrm{MgCl}_{2}$-Ti-isobutyl-DCPDMS-Mg2-Propylene-1,2-si-insertion-TS

$\begin{array}{lrrr}\text { Cl } & 6.50098600 & -2.90712400 & -1.37132800 \\ \text { Mg } & 5.28558500 & -4.53237100 & 0.09846000 \\ \text { Cl } & 7.75402800 & -1.04911400 & 1.24181200 \\ \text { Mg } & 5.64990400 & -0.91634600 & -0.11189800 \\ \text { Cl } & 3.18146400 & -4.39894700 & -1.25517600 \\ \text { Cl } & 0.22638700 & -2.27399400 & -1.34940700 \\ \text { Cl } & 3.54564800 & -0.78208500 & -1.46529800 \\ \text { Cl } & 1.11524800 & -4.03256800 & 1.47395800 \\ \text { Mg } & -0.98886500 & -3.89914600 & 0.12033200 \\ \text { Cl } & 4.43444800 & -2.54053700 & 1.35812700 \\ \text { Mg } & 2.33057800 & -2.40757500 & 0.00410300 \\ \text { Cl } & 1.47951900 & -0.41593400 & 1.26367400 \\ \text { Mg } & -0.62528300 & -0.28261400 & -0.09005400 \\ \text { Cl } & -3.09294100 & -3.76567100 & -1.23335500 \\ \text { Cl } & -6.04815700 & -1.64075200 & -1.32761700 \\ \text { Cl } & -2.72731700 & -0.08215700 & -1.49472100 \\ \text { Cl } & -5.15936700 & -3.39930200 & 1.49573300 \\ \text { Mg } & -7.26336500 & -3.26592400 & 0.14214300 \\ \text { Cl } & -1.83986700 & -1.90758400 & 1.37987200 \\ \text { Mg } & -3.94378200 & -1.77436600 & 0.02616100 \\ \text { Cl } & -4.71435600 & 0.27142300 & 1.33365300 \\ \text { Mg } & -6.89868000 & 0.35075500 & -0.06808700\end{array}$




\begin{tabular}{|c|c|c|c|}
\hline $\mathrm{Cl}$ & -9.00326800 & 0.48411500 & -1.42175100 \\
\hline $\mathrm{Cl}$ & -8.11438500 & -1.27439500 & 1.40157100 \\
\hline $\mathrm{Ti}$ & -3.50332800 & 2.11189700 & -0.16281900 \\
\hline $\mathrm{Cl}$ & -5.77803700 & 2.26608500 & -1.10169200 \\
\hline $\mathrm{Cl}$ & -1.29478500 & 1.85846200 & 0.88082800 \\
\hline$C$ & 4.21833500 & 3.11963200 & -0.64590000 \\
\hline C & 2.84578700 & 2.43877800 & -0.90627200 \\
\hline C & 3.85532500 & 4.34330900 & 0.23275400 \\
\hline $\mathrm{H}$ & 4.60449200 & 3.51850300 & -1.60446200 \\
\hline C & 1.77227700 & 3.54834000 & -0.71562800 \\
\hline $\mathrm{H}$ & 2.67781800 & 1.62034200 & -0.19428600 \\
\hline $\mathrm{H}$ & 2.81281300 & 1.97742400 & -1.90234700 \\
\hline C & 2.54735100 & 4.84217100 & -0.39563700 \\
\hline $\mathrm{H}$ & 4.64561400 & 5.10965600 & 0.26050000 \\
\hline $\mathrm{H}$ & 3.66818100 & 4.02863800 & 1.27648100 \\
\hline $\mathrm{H}$ & 1.12666300 & 3.66442000 & -1.60084400 \\
\hline $\mathrm{H}$ & 1.11191700 & 3.28205200 & 0.12410500 \\
\hline $\mathrm{H}$ & 2.78332100 & 5.38839000 & -1.32559900 \\
\hline $\mathrm{H}$ & 1.98850200 & 5.53406200 & 0.25492300 \\
\hline C & 6.89638700 & 3.11678400 & 0.86445600 \\
\hline C & 8.11559800 & 2.42610700 & 1.51458600 \\
\hline C & 7.57239300 & 4.07306100 & -0.15737200 \\
\hline $\mathrm{H}$ & 6.32384500 & 3.69920900 & 1.61032900 \\
\hline C & 9.08640100 & 2.17740700 & 0.34352400 \\
\hline $\mathrm{H}$ & 7.88233400 & 1.49095300 & 2.04228900 \\
\hline $\mathrm{H}$ & 8.55988000 & 3.12937000 & 2.24181200 \\
\hline C & 8.77407300 & 3.27175500 & -0.71517800 \\
\hline $\mathrm{H}$ & 7.93513500 & 4.96171400 & 0.38651700 \\
\hline $\mathrm{H}$ & 6.90018500 & 4.44967800 & -0.94712700 \\
\hline $\mathrm{H}$ & 10.13554900 & 2.20000600 & 0.67550400 \\
\hline $\mathrm{H}$ & 8.90118100 & 1.17021200 & -0.05728300 \\
\hline $\mathrm{H}$ & 9.63228600 & 3.93491400 & -0.90604000 \\
\hline $\mathrm{H}$ & 8.52190500 & 2.81123600 & -1.68378100 \\
\hline 0 & 5.02547600 & 0.79752700 & 1.05450800 \\
\hline 0 & 6.17588600 & 0.93669300 & -1.10458700 \\
\hline C & 6.24739600 & 1.10266600 & -2.51825500 \\
\hline $\mathrm{H}$ & 5.25910100 & 0.92962100 & -2.97215600 \\
\hline $\mathrm{H}$ & 6.61069700 & 2.10838400 & -2.78254500 \\
\hline $\mathrm{H}$ & 6.95561500 & 0.35823900 & -2.90900400 \\
\hline C & 4.91564200 & 0.75872600 & 2.48031700 \\
\hline $\mathrm{H}$ & 3.91782900 & 0.37551800 & 2.73624200 \\
\hline $\mathrm{H}$ & 5.70014100 & 0.08976500 & 2.86462000 \\
\hline $\mathrm{H}$ & 5.04447500 & 1.76298000 & 2.91444900 \\
\hline Si & 5.58904800 & 2.04187500 & 0.05563200 \\
\hline
\end{tabular}




$\begin{array}{lrrr}\text { C } & -3.78855100 & 3.99271800 & 0.86707000 \\ \text { H } & -2.92491600 & 4.38555900 & 1.42389100 \\ \text { H } & -4.07808100 & 3.05045300 & 1.43093000 \\ \text { C } & -4.99428600 & 4.93660700 & 0.90353100 \\ \text { C } & -5.86875000 & 4.62065800 & 2.13029400 \\ \text { H } & -6.74836900 & 5.28313100 & 2.16599300 \\ \text { H } & -5.30362800 & 4.75535000 & 3.06815900 \\ \text { H } & -6.23432900 & 3.58153400 & 2.09893500 \\ \text { C } & -2.69226800 & 3.14028200 & -1.93584900 \\ \text { H } & -3.44164300 & 2.84013100 & -2.67466700 \\ \text { H } & -1.68233600 & 2.73978400 & -2.07082800 \\ \text { C } & -2.85910800 & 4.33369500 & -1.22092200 \\ \text { H } & -3.77302300 & 4.90049900 & -1.41620600 \\ \text { C } & -1.66261300 & 5.11209200 & -0.74123300 \\ \text { H } & -1.91828400 & 5.88557800 & -0.00699300 \\ \text { H } & -0.90780900 & 4.44216600 & -0.30971200 \\ \text { H } & -1.21037300 & 5.61195700 & -1.61515500 \\ \text { H } & -5.61241900 & 4.73614500 & 0.01291100 \\ \text { C } & -4.58853700 & 6.41543600 & 0.89687400 \\ \text { H } & -4.04535700 & 6.70008100 & -0.01683100 \\ \text { H } & -5.47932800 & 7.06002100 & 0.95873200 \\ \text { H } & -3.94332800 & 6.65168800 & 1.76047000\end{array}$

$\mathrm{MgCl}_{2}$-Ti-isobuty1-DCPDMS-Mg2-Propylene-2,1-re-coordination

$\begin{array}{lrrr}\text { Cl } & -6.25287800 & -3.13091300 & 1.30787400 \\ \text { Mg } & -4.97251500 & -4.67972900 & -0.18904900 \\ \text { Cl } & -7.57966500 & -1.27969200 & -1.27351900 \\ \text { Mg } & -5.48284900 & -1.08562500 & 0.08413100 \\ \text { Cl } & -2.87571100 & -4.48499900 & 1.16852100 \\ \text { Cl } & -0.00901100 & -2.24424500 & 1.30237800 \\ \text { Cl } & -3.38594600 & -0.89005600 & 1.44146400 \\ \text { Cl } & -0.82559700 & -3.98768500 & -1.55202600 \\ \text { Mg } & 1.27119800 & -3.79295100 & -0.19445700 \\ \text { Cl } & -4.20245200 & -2.63337600 & -1.41299900 \\ \text { Mg } & -2.10589000 & -2.43914300 & -0.05505800 \\ \text { Cl } & -1.33589500 & -0.39298900 & -1.27894900 \\ \text { Mg } & 0.76125100 & -0.19833400 & 0.07879000 \\ \text { Cl } & 3.36794500 & -3.59818500 & 1.16313500 \\ \text { Cl } & 6.23472400 & -1.35743500 & 1.29706600 \\ \text { Cl } & 2.88303300 & 0.02816000 & 1.46560000 \\ \text { Cl } & 5.41824600 & -3.10088900 & -1.55735100 \\ \text { Mg } & 7.51495600 & -2.90618100 & -0.19983500 \\ \text { Cl } & 2.04113700 & -1.74690600 & -1.41826200 \\ \text { Mg } & 4.13785200 & -1.55224000 & -0.06079600\end{array}$




\begin{tabular}{|c|c|c|c|}
\hline $\mathrm{Cl}$ & 4.85585000 & 0.56998100 & -1.33770200 \\
\hline Mg & 7.00439100 & 0.68845900 & 0.07322900 \\
\hline $\mathrm{Cl}$ & 9.10147000 & 0.88325700 & 1.43081000 \\
\hline $\mathrm{Cl}$ & 8.28486300 & -0.86014900 & -1.42358600 \\
\hline $\mathrm{Ti}$ & 3.59517800 & 2.33814100 & 0.08240700 \\
\hline $\mathrm{Cl}$ & 5.70862700 & 2.44618500 & 1.26544600 \\
\hline $\mathrm{Cl}$ & 1.21706400 & 2.06606900 & -0.58253900 \\
\hline C & -4.27218000 & 3.01608300 & 0.69038500 \\
\hline C & -2.81005000 & 2.45889400 & 0.71340900 \\
\hline C & -4.17200300 & 4.35514000 & -0.09239700 \\
\hline $\mathrm{H}$ & -4.56955800 & 3.26891200 & 1.72491600 \\
\hline C & -1.91500900 & 3.60654100 & 0.17702400 \\
\hline $\mathrm{H}$ & -2.69677800 & 1.55501500 & 0.10295300 \\
\hline $\mathrm{H}$ & -2.53412800 & 2.16472500 & 1.73735800 \\
\hline C & -2.77535400 & 4.87310800 & 0.26455200 \\
\hline $\mathrm{H}$ & -4.98426200 & 5.05701800 & 0.15175400 \\
\hline $\mathrm{H}$ & -4.21760000 & 4.17823000 & -1.18268100 \\
\hline $\mathrm{H}$ & -0.96172100 & 3.69396200 & 0.71791300 \\
\hline $\mathrm{H}$ & -1.64931600 & 3.40918400 & -0.87439400 \\
\hline $\mathrm{H}$ & -2.78175000 & 5.26438100 & 1.29800700 \\
\hline $\mathrm{H}$ & -2.42914900 & 5.68585100 & -0.39422700 \\
\hline C & -7.03424600 & 2.88647000 & -0.65323100 \\
\hline C & -8.23709400 & 2.14790100 & -1.27770200 \\
\hline C & -7.69456900 & 3.71049400 & 0.49526000 \\
\hline $\mathrm{H}$ & -6.56427900 & 3.56731300 & -1.38494000 \\
\hline C & -9.08105300 & 1.72791400 & -0.06575000 \\
\hline $\mathrm{H}$ & -7.96583700 & 1.28229900 & -1.89757600 \\
\hline $\mathrm{H}$ & -8.79598700 & 2.86320300 & -1.90885600 \\
\hline C & -8.91050000 & 2.86632800 & 0.97219000 \\
\hline $\mathrm{H}$ & -8.03909200 & 4.67474700 & 0.08760100 \\
\hline $\mathrm{H}$ & -7.00471300 & 3.96297600 & 1.31841700 \\
\hline $\mathrm{H}$ & -10.13346900 & 1.54748400 & -0.33253000 \\
\hline $\mathrm{H}$ & -8.68373700 & 0.77691600 & 0.31811000 \\
\hline $\mathrm{H}$ & -9.80922400 & 3.50004000 & 1.03378600 \\
\hline $\mathrm{H}$ & -8.75204500 & 2.46120600 & 1.98450600 \\
\hline 0 & -4.98847500 & 0.69995600 & -1.03172100 \\
\hline 0 & -6.08796100 & 0.68912100 & 1.15415200 \\
\hline C & -6.16862300 & 0.80902100 & 2.57126400 \\
\hline $\mathrm{H}$ & -5.16528100 & 0.72798200 & 3.01825700 \\
\hline $\mathrm{H}$ & -6.63482500 & 1.76377400 & 2.86249200 \\
\hline $\mathrm{H}$ & -6.79192600 & -0.01646100 & 2.94341500 \\
\hline C & -4.88103500 & 0.72355400 & -2.45761700 \\
\hline $\mathrm{H}$ & -3.87514600 & 0.37266700 & -2.72935600 \\
\hline $\mathrm{H}$ & -5.65154700 & 0.05467200 & -2.86986500 \\
\hline
\end{tabular}




$\begin{array}{lrrr}\text { H } & -5.02919400 & 1.74300100 & -2.84883200 \\ \text { Si } & -5.60682400 & 1.87144500 & 0.02155000 \\ \text { C } & 3.74339400 & 3.79409500 & -1.36414700 \\ \text { H } & 4.65865600 & 4.22099800 & -0.89498600 \\ \text { H } & 4.07547500 & 3.23893400 & -2.26172200 \\ \text { C } & 2.69886700 & 4.86895500 & -1.71004100 \\ \text { C } & 1.98133100 & 4.53231200 & -3.02244700 \\ \text { C } & 3.32761000 & 6.26918700 & -1.76109800 \\ \text { H } & 1.92646800 & 4.88436000 & -0.91914300 \\ \text { H } & 1.18023100 & 5.25918900 & -3.23362900 \\ \text { H } & 1.52950600 & 3.53029300 & -2.98259700 \\ \text { H } & 2.69034100 & 4.55541500 & -3.86787500 \\ \text { H } & 2.57157600 & 7.03782700 & -1.99302200 \\ \text { H } & 4.10937800 & 6.31809900 & -2.53778100 \\ \text { H } & 3.79597800 & 6.53583000 & -0.79887300 \\ \text { C } & 2.57157800 & 3.19560400 & 2.57284700 \\ \text { H } & 3.36986100 & 2.68258000 & 3.12469200 \\ \text { C } & 2.91672200 & 4.22497800 & 1.76755900 \\ \text { H } & 2.15577300 & 4.78670700 & 1.21745600 \\ \text { H } & 3.94329900 & 4.59869600 & 1.73394900 \\ \text { C } & 1.17312800 & 2.74652700 & 2.85445500 \\ \text { H } & 1.09272000 & 1.64971700 & 2.83151900 \\ \text { H } & 0.90099900 & 3.05843400 & 3.87820300 \\ \text { H } & 0.44854100 & 3.17790800 & 2.15176700\end{array}$

$\mathrm{MgCl}_{2}$-Ti-isobutyl-DCPDMS-Mg2-Propylene-2,1-re-insertion-TS

$\begin{array}{lrrr}\text { Cl } & 6.25029400 & -3.09836700 & -1.46864600 \\ \text { Mg } & 5.00234700 & -4.68338700 & 0.01771800 \\ \text { Cl } & 7.61911400 & -1.29888800 & 1.12733900 \\ \text { Mg } & 5.49700900 & -1.08254300 & -0.18696100 \\ \text { Cl } & 2.88025700 & -4.46638200 & -1.29648900 \\ \text { Cl } & 0.00537400 & -2.23281500 & -1.32881900 \\ \text { Cl } & 3.37482100 & -0.86470600 & -1.50091000 \\ \text { Cl } & 0.87930900 & -4.03473400 & 1.47146200 \\ \text { Mg } & -1.24274500 & -3.81774500 & 0.15730400 \\ \text { Cl } & 4.24903200 & -2.66650500 & 1.29963500 \\ \text { Mg } & 2.12721800 & -2.44993900 & -0.01500700 \\ \text { Cl } & 1.37394900 & -0.43330600 & 1.26687800 \\ \text { Mg } & -0.74955300 & -0.21648300 & -0.04767300 \\ \text { Cl } & -3.36481100 & -3.60068500 & -1.15697700 \\ \text { Cl } & -6.24010100 & -1.36709000 & -1.18959200 \\ \text { Cl } & -2.88558300 & -0.02265000 & -1.47472800 \\ \text { Cl } & -5.36595700 & -3.16903800 & 1.61092200 \\ \text { Mg } & -7.48795400 & -2.95206000 & 0.29676000\end{array}$




\begin{tabular}{|c|c|c|c|}
\hline $\mathrm{Cl}$ & -1.99593300 & -1.80115800 & 1.43905200 \\
\hline Mg & -4.11778000 & -1.58430500 & 0.12473200 \\
\hline $\mathrm{Cl}$ & -4.75899400 & 0.53508800 & 1.37576906 \\
\hline Mg & -6.99259600 & 0.64946100 & 0.09244800 \\
\hline $\mathrm{Cl}$ & -9.11539500 & 0.86637500 & -1.2220300 \\
\hline $\mathrm{Cl}$ & -8.24113300 & -0.93551800 & 1.5784260 \\
\hline Ti & -3.53845200 & 2.25326800 & -0.3274470 \\
\hline $\mathrm{Cl}$ & -5.84148400 & 2.51920300 & -1.0495300 \\
\hline $\mathrm{Cl}$ & -1.30596100 & 1.98724200 & 0.7404940 \\
\hline C & 4.26170700 & 3.06390100 & $-0.4820810 e$ \\
\hline C & 2.87490200 & 2.47732100 & -0.83205100 \\
\hline C & 3.91399900 & 4.13226200 & 0.5836250 \\
\hline $\mathrm{H}$ & 4.66145100 & 3.59822300 & -1.36675100 \\
\hline C & 1.88232900 & 3.66703900 & -0.70925300 \\
\hline $\mathrm{H}$ & 2.61545200 & 1.68522500 & -0.11429300 \\
\hline $\mathrm{H}$ & 2.85968300 & 1.99930300 & -1.82120100 \\
\hline C & 2.64746800 & 4.79939600 & 0.02279200 \\
\hline $\mathrm{H}$ & 4.72824300 & 4.84969700 & 0.76886100 \\
\hline $\mathrm{H}$ & 3.68506100 & 3.63996100 & 1.54699700 \\
\hline $\mathrm{H}$ & 1.54003300 & 4.00804900 & -1.69889800 \\
\hline $\mathrm{H}$ & 0.98681900 & 3.36242000 & -0.14790600 \\
\hline $\mathrm{H}$ & 2.94481800 & 5.58399300 & -0.69296500 \\
\hline $\mathrm{H}$ & 2.04592200 & 5.29035400 & 0.80418600 \\
\hline C & 6.97857800 & 2.84862900 & 0.90062600 \\
\hline C & 8.19144000 & 2.09331100 & 1.48500500 \\
\hline C & 7.65946200 & 3.81987200 & -0.10373800 \\
\hline $\mathrm{H}$ & 6.45495100 & 3.42071200 & 1.68902400 \\
\hline C & 9.12514300 & 1.86283300 & 0.27932900 \\
\hline $\mathrm{H}$ & 7.93736200 & 1.14636300 & 1.97942300 \\
\hline $\mathrm{H}$ & 8.67700900 & 2.75164200 & 2.22753800 \\
\hline C & 8.79522900 & 2.98651800 & -0.74306300 \\
\hline $\mathrm{H}$ & 8.08772600 & 4.66294200 & 0.46496800 \\
\hline $\mathrm{H}$ & 6.97901900 & 4.26644900 & -0.84865700 \\
\hline $\mathrm{H}$ & 10.18346300 & 1.86922800 & 0.58190800 \\
\hline $\mathrm{H}$ & 8.91965900 & 0.86771300 & -0.14105700 \\
\hline $\mathrm{H}$ & 9.66557400 & 3.61922200 & -0.97748500 \\
\hline $\mathrm{H}$ & 8.46524100 & 2.55188000 & -1.70019700 \\
\hline 0 & 4.97288900 & 0.62478500 & 1.05390600 \\
\hline 0 & 6.06740500 & 0.78475200 & -1.13294700 \\
\hline C & 6.11838400 & 1.01458100 & -2.53861500 \\
\hline $\mathrm{H}$ & 5.14221900 & 0.78159400 & -2.99179500 \\
\hline $\mathrm{H}$ & 6.39270300 & 2.05756200 & -2.76311900 \\
\hline $\mathrm{H}$ & 6.88327200 & 0.34977900 & $-2.965144 €$ \\
\hline c & 4.87624700 & 0.54821200 & $2.478615 e$ \\
\hline
\end{tabular}




$\begin{array}{lrrr}\text { H } & 3.86733000 & 0.19473700 & 2.73395300 \\ \text { H } & 5.63893700 & -0.15940600 & 2.83650300 \\ \text { H } & 5.04462700 & 1.53449400 & 2.93982200 \\ \text { Si } & 5.59283600 & 1.87108200 & 0.09301400 \\ \text { C } & -3.60915200 & 4.22095300 & 0.56241000 \\ \text { H } & -4.52823600 & 4.79796300 & 0.38608600 \\ \text { H } & -3.94492600 & 3.37376900 & 1.24583600 \\ \mathrm{C} & -2.52658800 & 5.04177700 & 1.27109000 \\ \mathrm{C} & -2.52523200 & 4.75828100 & 2.78223200 \\ \mathrm{C} & -2.69317300 & 6.54162400 & 0.99466500 \\ \mathrm{H} & -1.54474700 & 4.72073600 & 0.88377400 \\ \mathrm{H} & -1.74348900 & 5.34715200 & 3.28855000 \\ \mathrm{H} & -2.32824200 & 3.69386900 & 2.98261900 \\ \mathrm{H} & -3.49491500 & 5.02497000 & 3.23563400 \\ \mathrm{H} & -1.87588100 & 7.11756700 & 1.45669100 \\ \mathrm{H} & -3.64467800 & 6.91199000 & 1.41241100 \\ \mathrm{H} & -2.69384500 & 6.77033100 & -0.08283700 \\ \mathrm{C} & -2.73894500 & 3.09000700 & -2.26985000 \\ \mathrm{H} & -3.51510300 & 2.62417500 & -2.89333500 \\ \mathrm{C} & -3.08317400 & 4.28992400 & -1.64745800 \\ \mathrm{H} & -2.28897800 & 4.97619500 & -1.34799400 \\ \mathrm{H} & -4.06151800 & 4.73591100 & -1.83192900 \\ \mathrm{C} & -1.30509300 & 2.74624300 & -2.61013000 \\ \mathrm{H} & -1.15150600 & 1.66170400 & -2.70205100 \\ \mathrm{H} & -1.03489800 & 3.19128700 & -3.58362600 \\ \mathrm{H} & -0.60985800 & 3.13167000 & -1.85469900\end{array}$

$\mathrm{MgCl}_{2}$-Ti-isobutyl-DCPDMS-Mg2-Propylene-2,1-si-coordination

$\begin{array}{lrrr}\text { Cl } & -6.31372900 & -3.09869600 & 1.31182600 \\ \text { Mg } & -5.03878200 & -4.65755900 & -0.17928000 \\ \text { Cl } & -7.62981200 & -1.24923300 & -1.27629100 \\ \text { Mg } & -5.53346800 & -1.06044500 & 0.08283500 \\ \text { Cl } & -2.94245000 & -4.46810900 & 1.17976500 \\ \text { Cl } & -0.06597100 & -2.23967300 & 1.30985400 \\ \text { Cl } & -3.43703300 & -0.87015600 & 1.44163700 \\ \text { Cl } & -0.88752700 & -3.98787800 & -1.54022300 \\ \text { Mg } & 1.20879300 & -3.79842100 & -0.18117700 \\ \text { Cl } & -4.25848600 & -2.61824500 & -1.40848300 \\ \text { Mg } & -2.16240800 & -2.42927800 & -0.04905700 \\ \text { Cl } & -1.38217700 & -0.39016900 & -1.27821100 \\ \text { Mg } & 0.71460400 & -0.20070700 & 0.08100700 \\ \text { Cl } & 3.30509000 & -3.60894300 & 1.17789100 \\ \text { Cl } & 6.18161100 & -1.38048300 & 1.30803500 \\ \text { Cl } & 2.83224700 & 0.05519000 & 1.45046200\end{array}$




\begin{tabular}{|c|c|c|c|}
\hline $\mathrm{Cl}$ & 5.36017400 & -3.12872900 & -1.54204000 \\
\hline Mg & 7.45643700 & -2.93929000 & -0.18303800 \\
\hline $\mathrm{Cl}$ & 1.98896000 & -1.75943100 & -1.41023700 \\
\hline Mg & 4.08525400 & -1.57011100 & -0.05138600 \\
\hline $\mathrm{Cl}$ & 4.81812400 & 0.51281300 & -1.35410300 \\
\hline Mg & 6.96132000 & 0.65833200 & 0.07897300 \\
\hline $\mathrm{Cl}$ & 9.05810100 & 0.84788200 & 1.43801800 \\
\hline $\mathrm{Cl}$ & 8.23656300 & -0.90030500 & -1.41204300 \\
\hline $\mathrm{Ti}$ & 3.57365900 & 2.34852500 & -0.01644800 \\
\hline $\mathrm{Cl}$ & 5.68912800 & 2.49341000 & 1.15686200 \\
\hline $\mathrm{Cl}$ & 1.16862900 & 2.06710000 & -0.60793500 \\
\hline C & -4.22880100 & 2.96892600 & 0.87225400 \\
\hline C & -2.77790000 & 2.40278200 & 0.94274400 \\
\hline C & -4.05394600 & 4.36070000 & 0.21222800 \\
\hline $\mathrm{H}$ & -4.58143300 & 3.15238300 & 1.90545700 \\
\hline C & -1.83280500 & 3.63260300 & 0.81002600 \\
\hline $\mathrm{H}$ & -2.59208500 & 1.67730000 & 0.13940500 \\
\hline $\mathrm{H}$ & -2.62384600 & 1.84839500 & 1.87835300 \\
\hline C & -2.74643600 & 4.87124400 & 0.82845700 \\
\hline $\mathrm{H}$ & -4.91096600 & 5.03123000 & 0.38091500 \\
\hline $\mathrm{H}$ & -3.92987300 & 4.26124500 & -0.88229700 \\
\hline $\mathrm{H}$ & -1.06974500 & 3.66547400 & 1.60469400 \\
\hline $\mathrm{H}$ & -1.28415200 & 3.58166400 & -0.14314300 \\
\hline $\mathrm{H}$ & -2.94033300 & 5.19220300 & 1.86720100 \\
\hline $\mathrm{H}$ & -2.32025800 & 5.73528100 & 0.29362600 \\
\hline C & -6.91939000 & 2.96210200 & -0.63938900 \\
\hline C & -8.06922900 & 2.21985100 & -1.36622100 \\
\hline C & -7.61774700 & 3.74305700 & 0.53394000 \\
\hline $\mathrm{H}$ & -6.41763200 & 3.67005500 & -1.32109900 \\
\hline C & -9.06728500 & 1.91600300 & -0.24550000 \\
\hline $\mathrm{H}$ & -7.75974900 & 1.30429700 & -1.88869300 \\
\hline $\mathrm{H}$ & -8.52804200 & 2.90017400 & -2.10633500 \\
\hline C & -9.07331200 & 3.20584000 & 0.58998000 \\
\hline $\mathrm{H}$ & -7.59459700 & 4.82931300 & 0.35607300 \\
\hline $\mathrm{H}$ & -7.10726100 & 3.59689900 & 1.50220000 \\
\hline $\mathrm{H}$ & -10.06368800 & 1.63973200 & -0.62310900 \\
\hline $\mathrm{H}$ & -8.69611400 & 1.05635900 & 0.33538800 \\
\hline $\mathrm{H}$ & -9.75368800 & 3.94146400 & 0.12911600 \\
\hline $\mathrm{H}$ & -9.42483900 & 3.05170600 & 1.62273500 \\
\hline 0 & -4.95684900 & 0.72882700 & -0.99512200 \\
\hline 0 & -6.13948700 & 0.70627600 & 1.14777200 \\
\hline C & -6.28229100 & 0.76590200 & 2.56280200 \\
\hline $\mathrm{H}$ & -5.29731700 & 0.84909700 & 3.04906400 \\
\hline $\mathrm{H}$ & -6.92490000 & 1.61064100 & 2.85837500 \\
\hline
\end{tabular}




$\begin{array}{lrrr}\text { H } & -6.75695600 & -0.17032900 & 2.88933600 \\ \text { C } & -4.84625300 & 0.76897400 & -2.42063900 \\ \text { H } & -3.83688400 & 0.43130600 & -2.69524700 \\ \text { H } & -5.60874800 & 0.09635700 & -2.84192700 \\ \text { H } & -5.00728300 & 1.79007300 & -2.80198300 \\ \text { Si } & -5.55370100 & 1.89791500 & 0.07320200 \\ \text { C } & 3.76343000 & 3.73216600 & -1.53393900 \\ \text { H } & 4.56003200 & 4.27239600 & -0.97232600 \\ \text { H } & 4.27066400 & 3.17277900 & -2.34253800 \\ \text { C } & 2.70088200 & 4.68619500 & -2.09747800 \\ \text { C } & 2.04309700 & 4.09375200 & -3.34942700 \\ \text { C } & 3.29360000 & 6.07351400 & -2.38956600 \\ \text { H } & 1.90349900 & 4.82076700 & -1.34239500 \\ \text { H } & 1.23212700 & 4.74388600 & -3.71553000 \\ \text { H } & 1.61718800 & 3.10065200 & -3.14712400 \\ \text { H } & 2.78365900 & 3.99008000 & -4.16112500 \\ \text { H } & 2.52627100 & 6.75847600 & -2.78734300 \\ \text { H } & 4.10282700 & 6.00232500 & -3.13586100 \\ \text { H } & 3.71666000 & 6.53105500 & -1.47987800 \\ \text { C } & 2.46468100 & 3.30239200 & 2.39292500 \\ \text { C } & 2.86200800 & 4.26573700 & 1.53174000 \\ \text { H } & 2.19444300 & 4.60701600 & 0.73473100 \\ \text { H } & 3.79885700 & 4.81151400 & 1.67876100 \\ \text { H } & 1.48909100 & 2.83237400 & 2.21752200 \\ \text { C } & 3.21864600 & 2.85578800 & 3.60351200 \\ \text { H } & 2.64259800 & 3.12903400 & 4.50523900 \\ \text { H } & 3.32723200 & 1.76061200 & 3.61504100 \\ \text { H } & 4.21603600 & 3.31226100 & 3.66196400\end{array}$

$\mathrm{MgCl}_{2}$-Ti-isobutyl-DCPDMS-Mg2-Propylene-2,1-si-insertion-TS

$\begin{array}{lrrr}\text { Cl } & 6.35717400 & -3.05933500 & -1.39047600 \\ \text { Mg } & 5.11354400 & -4.63374300 & 0.11071100 \\ \text { Cl } & 7.68299800 & -1.20271900 & 1.18752900 \\ \text { Mg } & 5.57041900 & -1.03142700 & -0.14858200 \\ \text { Cl } & 3.00097200 & -4.46178900 & -1.22531800 \\ \text { Cl } & 0.10172000 & -2.26119300 & -1.31950300 \\ \text { Cl } & 3.45773900 & -0.85863600 & -1.48436500 \\ \text { Cl } & 0.97086100 & -4.00744400 & 1.51765300 \\ \text { Mg } & -1.14169900 & -3.83548100 & 0.18162100 \\ \text { Cl } & 4.32674600 & -2.60477300 & 1.35282000 \\ \text { Mg } & 2.21442700 & -2.43330000 & 0.01640700 \\ \text { Cl } & 1.42769800 & -0.40453000 & 1.25846100 \\ \text { Mg } & -0.68525200 & -0.23254400 & -0.07773500 \\ \text { Cl } & -3.25423700 & -3.66349300 & -1.15444100\end{array}$




\begin{tabular}{|c|c|c|c|}
\hline $\mathrm{Cl}$ & -6.15348400 & -1.46286300 & -1.24865200 \\
\hline $\mathrm{Cl}$ & -2.79199200 & -0.01381100 & -1.47871900 \\
\hline $\mathrm{Cl}$ & -5.28451600 & -3.20917700 & 1.58847600 \\
\hline Mg & -7.39702300 & -3.03722200 & 0.25249900 \\
\hline $\mathrm{Cl}$ & -1.92838300 & -1.80683100 & 1.4236040 \\
\hline Mg & -4.04093500 & -1.63503500 & 0.0877920 \\
\hline $\mathrm{Cl}$ & -4.72979400 & 0.45512800 & 1.3543700 \\
\hline Mg & -6.93971900 & 0.56555700 & -0.00671600 \\
\hline $\mathrm{Cl}$ & -9.05272600 & 0.73764800 & -1.34272400 \\
\hline $\mathrm{Cl}$ & -8.18362600 & -1.00858100 & 1.4944240 \\
\hline $\mathrm{Ti}$ & -3.49825900 & 2.24439600 & -0.20528300 \\
\hline $\mathrm{Cl}$ & -5.79195400 & 2.48276100 & -1.04107800 \\
\hline $\mathrm{Cl}$ & -1.31216000 & 1.91215500 & 0.8804130 \\
\hline C & 4.16362200 & 3.03080300 & -0.69491800 \\
\hline C & 2.76973400 & 2.38881200 & -0.94151900 \\
\hline C & 3.83995300 & 4.26768100 & 0.18063500 \\
\hline $\mathrm{H}$ & 4.55242400 & 3.41561600 & -1.65844100 \\
\hline C & 1.72997000 & 3.53566300 & -0.76647600 \\
\hline $\mathrm{H}$ & 2.58181500 & 1.58774500 & -0.21481200 \\
\hline $\mathrm{H}$ & 2.72050900 & 1.91131400 & -1.92951900 \\
\hline C & 2.54615800 & 4.80469400 & -0.44578900 \\
\hline $\mathrm{H}$ & 4.65275200 & 5.01012600 & 0.20426000 \\
\hline H & 3.64679600 & 3.96084400 & 1.22560700 \\
\hline $\mathrm{H}$ & 1.09854500 & 3.67016500 & -1.66032700 \\
\hline $\mathrm{H}$ & 1.04920900 & 3.29873900 & 0.06559700 \\
\hline $\mathrm{H}$ & 2.79756000 & 5.34481100 & -1.37518900 \\
\hline $\mathrm{H}$ & 2.01034300 & 5.51284100 & 0.20667800 \\
\hline C & 6.84993900 & 2.99440500 & 0.80220200 \\
\hline C & 8.06038500 & 2.28103400 & 1.44475300 \\
\hline C & 7.53719000 & 3.94100700 & -0.22107000 \\
\hline $\mathrm{H}$ & 6.29215100 & 3.58522100 & 1.55250100 \\
\hline C & 9.02178600 & 2.01918600 & 0.26887000 \\
\hline $\mathrm{H}$ & 7.81461700 & 1.34811100 & 1.97093700 \\
\hline $\mathrm{H}$ & 8.51983800 & 2.97414600 & 2.17226100 \\
\hline C & 8.72392000 & 3.12187300 & -0.78520700 \\
\hline $\mathrm{H}$ & 7.91622300 & 4.82312400 & 0.32240300 \\
\hline $\mathrm{H}$ & 6.86821400 & 4.32954800 & -1.00784300 \\
\hline $\mathrm{H}$ & 10.07250900 & 2.02243200 & 0.59653800 \\
\hline $\mathrm{H}$ & 8.81778100 & 1.01650900 & -0.13395600 \\
\hline $\mathrm{H}$ & 9.59226100 & 3.77150900 & -0.97673000 \\
\hline $\mathrm{H}$ & 8.46103100 & 2.66876200 & -1.75434800 \\
\hline 0 & 4.96561400 & 0.69863100 & 1.01399300 \\
\hline 0 & 6.10346600 & 0.82023000 & -1.15341800 \\
\hline C & 6.16704900 & 0.97988700 & -2.56816900 \\
\hline & & & S101 \\
\hline
\end{tabular}




$\begin{array}{lrrr}\text { H } & 5.17282400 & 0.82218500 & -3.01470200 \\ \text { H } & 6.54560400 & 1.97831600 & -2.83882500 \\ \text { H } & 6.85980800 & 0.22235600 & -2.96150200 \\ \text { C } & 4.86951900 & 0.67163000 & 2.44103100 \\ \text { H } & 3.86708300 & 0.31042400 & 2.71023600 \\ \text { H } & 5.64429900 & -0.01046800 & 2.82195300 \\ \text { H } & 5.02366100 & 1.67573000 & 2.86717200 \\ \text { Si } & 5.52428200 & 1.93447000 & 0.00256400 \\ \mathrm{C} & -3.70016000 & 4.17075400 & 0.74023500 \\ \text { H } & -4.58663600 & 4.74758600 & 0.43645300 \\ \text { H } & -4.11403200 & 3.28048000 & 1.31215500 \\ \mathrm{C} & -2.77791300 & 4.95447100 & 1.67479800 \\ \mathrm{C} & -3.17467500 & 4.70869300 & 3.14111700 \\ \mathrm{C} & -2.78223100 & 6.45400300 & 1.35195300 \\ \mathrm{H} & -1.75131000 & 4.56760100 & 1.55234600 \\ \mathrm{H} & -2.51677400 & 5.27178100 & 3.82255200 \\ \mathrm{H} & -3.09282100 & 3.64092200 & 3.39998000 \\ \mathrm{H} & -4.21242000 & 5.03105600 & 3.33055200 \\ \mathrm{H} & -2.09024000 & 6.99783300 & 2.01409900 \\ \mathrm{H} & -3.78980700 & 6.87968100 & 1.49608400 \\ \mathrm{H} & -2.47832200 & 6.66044500 & 0.31438400 \\ \mathrm{C} & -2.54236500 & 3.17407600 & -2.03541300 \\ \mathrm{C} & -2.61204700 & 4.31791000 & -1.24188000 \\ \mathrm{H} & -1.73487900 & 4.62256400 & -0.66939600 \\ \mathrm{H} & -3.32251000 & 5.10797900 & -1.49536900 \\ \mathrm{H} & -1.59745000 & 2.61898700 & -1.98864200 \\ \mathrm{C} & -3.37740200 & 2.96515400 & -3.27992200 \\ \mathrm{H} & -2.79933700 & 3.28205800 & -4.16593500 \\ \mathrm{H} & -3.64500400 & 1.90849600 & -3.42240100 \\ \mathrm{H} & -4.30856600 & 3.54737500 & -3.24649600\end{array}$

\section{$\mathrm{MgCl}_{2}$-Ti-isobutyl-CMDMS-Mg2-AlEt ${ }_{2} \mathrm{Cl}$}

$\begin{array}{lrrr}\text { Cl } & -8.05983600 & -1.66535500 & 1.49062600 \\ \text { Mg } & -7.09662600 & -3.53444200 & 0.12799000 \\ \text { Cl } & -9.06828900 & 0.17831200 & -1.23609900 \\ \text { Mg } & -6.95291700 & 0.10362300 & 0.10450800 \\ \text { Cl } & -4.98115800 & -3.60914100 & 1.46842300 \\ \text { Cl } & -1.75874200 & -1.91479800 & 1.42265500 \\ \text { Cl } & -4.83735900 & 0.02895100 & 1.44477000 \\ \text { Cl } & -2.91099900 & -3.70918100 & -1.28041300 \\ \text { Mg } & -0.79555100 & -3.78386600 & 0.06002700 \\ \text { Cl } & -5.98966500 & -1.76538200 & -1.25828100 \\ \text { Mg } & -3.87422700 & -1.84009200 & 0.08225500 \\ \text { Cl } & -2.76721700 & -0.07114300 & -1.30403900 \\ & & & \\ & & & \text { S102 }\end{array}$




\begin{tabular}{|c|c|c|c|}
\hline Mg & -0.65119300 & -0.14585200 & 0.03664700 \\
\hline $\mathrm{Cl}$ & 1.31985200 & -3.85851300 & 1.40047400 \\
\hline $\mathrm{Cl}$ & 4.54239600 & -2.16418800 & 1.35482700 \\
\hline $\mathrm{Cl}$ & 1.38975800 & -0.22059000 & 1.57548200 \\
\hline $\mathrm{Cl}$ & 3.39021100 & -3.95861900 & -1.34829800 \\
\hline Mg & 5.50557200 & -4.03333500 & -0.00791600 \\
\hline $\mathrm{Cl}$ & 0.31139100 & -2.01486600 & -1.32626000 \\
\hline Mg & 2.42690700 & -2.08962200 & 0.01379700 \\
\hline $\mathrm{Cl}$ & 3.41771200 & -0.17687700 & -1.31492200 \\
\hline Mg & 5.64869700 & -0.39533300 & -0.03155700 \\
\hline $\mathrm{Cl}$ & 7.76484500 & -0.46992500 & 1.30894600 \\
\hline $\mathrm{Cl}$ & 6.61258600 & -2.26423400 & -1.39420900 \\
\hline $\mathrm{Ti}$ & 2.55203100 & 1.65438100 & 0.31757700 \\
\hline $\mathrm{Cl}$ & 4.60616600 & 1.26958700 & 1.55361300 \\
\hline $\mathrm{Cl}$ & 0.36018900 & 1.84623300 & -0.84764400 \\
\hline $\mathrm{C}$ & -4.55885100 & 3.48206900 & 0.20267900 \\
\hline C & -3.60860500 & 3.62961100 & -1.02100900 \\
\hline C & -4.49663400 & 4.74441700 & 1.08220300 \\
\hline $\mathrm{H}$ & -4.20550000 & 2.62573700 & 0.80366300 \\
\hline C & -2.34663200 & 4.45582200 & -0.67794400 \\
\hline $\mathrm{H}$ & -4.12805900 & 4.11015100 & -1.87087400 \\
\hline $\mathrm{H}$ & -3.32030700 & 2.62356600 & -1.35898000 \\
\hline C & -3.08725400 & 4.89959800 & 1.70560400 \\
\hline $\mathrm{H}$ & -4.73336500 & 5.62610600 & 0.46059300 \\
\hline $\mathrm{H}$ & -5.25485800 & 4.72733700 & 1.88277300 \\
\hline C & -1.98263500 & 4.30815400 & 0.80191800 \\
\hline $\mathrm{H}$ & -2.51922900 & 5.52503200 & -0.89468200 \\
\hline $\mathrm{H}$ & -1.50862300 & 4.13710900 & -1.31696200 \\
\hline $\mathrm{H}$ & -3.06107400 & 4.40270700 & 2.68991400 \\
\hline $\mathrm{H}$ & -2.88802000 & 5.96841400 & 1.89340400 \\
\hline $\mathrm{H}$ & -1.84446500 & 3.23550300 & 1.02334800 \\
\hline $\mathrm{H}$ & -1.01308100 & 4.78685200 & 1.01842100 \\
\hline C & -7.35186000 & 4.10073100 & -1.23484300 \\
\hline $\mathrm{H}$ & -8.25702500 & 3.55903600 & -1.55310800 \\
\hline $\mathrm{H}$ & -7.64657900 & 4.92676200 & -0.56758000 \\
\hline $\mathrm{H}$ & -6.86266000 & 4.53871300 & -2.11928100 \\
\hline 0 & -6.04297200 & 1.47889400 & -1.25226900 \\
\hline 0 & -7.09033000 & 2.10147400 & 0.89127600 \\
\hline C & -6.03346500 & 1.31321500 & -2.67345900 \\
\hline $\mathrm{H}$ & -5.20588400 & 0.63826300 & -2.93137300 \\
\hline $\mathrm{H}$ & -6.99869900 & 0.87967200 & -2.97562100 \\
\hline $\mathrm{H}$ & -5.87838500 & 2.27871700 & -3.18057900 \\
\hline C & -7.14833300 & 2.43244100 & 2.27431100 \\
\hline $\mathrm{H}$ & -6.16241900 & 2.29863400 & 2.74782200 \\
\hline
\end{tabular}




$\begin{array}{lrrr}\text { H } & -7.49919500 & 3.46779100 & 2.41753700 \\ \text { H } & -7.86449100 & 1.74873100 & 2.75186500 \\ \text { Si } & -6.24177400 & 2.89726800 & -0.35544900 \\ \text { C } & 2.64927300 & 3.70162000 & 0.53395500 \\ \text { H } & 1.66231700 & 4.18137800 & 0.46983400 \\ \text { H } & 3.19724400 & 3.99194500 & 1.44202400 \\ \text { C } & 3.47006300 & 3.72174400 & -0.74181200 \\ \text { C } & 4.89306200 & 4.26088600 & -0.54664000 \\ \text { C } & 2.75351900 & 4.37682400 & -1.93038500 \\ \text { H } & 3.64197600 & 2.63503000 & -1.08486400 \\ \text { H } & 5.49878900 & 4.12356900 & -1.45416100 \\ \text { H } & 5.40225400 & 3.74343700 & 0.27679000 \\ \text { H } & 4.84331300 & 5.33610800 & -0.31207000 \\ \text { H } & 3.33146700 & 4.24889000 & -2.85893800 \\ \text { H } & 2.64238500 & 5.45617100 & -1.74037500 \\ \text { H } & 1.75256400 & 3.94902100 & -2.08360100 \\ \text { C } & 8.23353200 & 2.92715500 & 1.29034800 \\ \text { H } & 7.16816900 & 2.91991800 & 1.58353600 \\ \text { H } & 8.37741600 & 3.85912000 & 0.71096000 \\ \text { C } & 10.12795300 & 0.83890400 & -0.84807200 \\ \text { H } & 10.44522800 & 1.67962700 & -1.49497100 \\ \text { H } & 10.93776400 & 0.73259800 & -0.10000700 \\ \text { C } & 10.00558300 & -0.44932100 & -1.67987400 \\ \text { H } & 10.95253800 & -0.72382400 & -2.17771600 \\ \text { H } & 9.69971100 & -1.30464600 & -1.05472600 \\ \text { H } & 9.23818800 & -0.34932500 & -2.46534200 \\ \mathrm{C} & 9.11618900 & 2.94430700 & 2.55152200 \\ \text { H } & 10.19147200 & 2.98025700 & 2.30345300 \\ \text { H } & 8.90902100 & 3.81324500 & 3.20124000 \\ \text { H } & 8.95726100 & 2.04001600 & 3.16392800 \\ \text { Cl } & 6.74529000 & 1.33869600 & -1.41396000 \\ \text { Al } & 8.49929400 & 1.37670100 & 0.11119000\end{array}$

$\mathrm{MgCl}_{2}$-Ti-isobutyl-CMDMS-Mg2-AlEt ${ }_{2} \mathrm{Cl}$-Propylene-1, 2-re-coordination

$\begin{array}{lrrr}\text { Cl } & 8.11555000 & -1.69433600 & -1.52309700 \\ \text { Mg } & 7.19466300 & -3.59006100 & -0.16802300 \\ \text { Cl } & 9.11056700 & 0.14648500 & 1.21047400 \\ \text { Mg } & 6.98754500 & 0.04464900 & -0.11618100 \\ \text { Cl } & 5.07154800 & -3.69191200 & -1.49450400 \\ \text { Cl } & 1.82038500 & -2.05472600 & -1.41397900 \\ \text { Cl } & 4.86434100 & -0.05717600 & -1.44249100 \\ \text { Cl } & 3.02263100 & -3.84858200 & 1.26757100 \\ \text { Mg } & 0.89950600 & -3.95043600 & -0.05891700 \\ \text { Cl } & 6.06661900 & -1.85099700 & 1.23904800\end{array}$




\begin{tabular}{|c|c|c|c|}
\hline Mg & 3.94351600 & -1.95288500 & -0.08751000 \\
\hline $\mathrm{Cl}$ & 2.81546400 & -0.21385700 & 1.31952000 \\
\hline Mg & 0.69223700 & -0.31590900 & -0.00706100 \\
\hline $\mathrm{Cl}$ & -1.22353700 & -4.05222800 & -1.38541300 \\
\hline $\mathrm{Cl}$ & -4.47479300 & -2.41507600 & -1.30493600 \\
\hline $\mathrm{Cl}$ & -1.36098400 & -0.46024600 & -1.47480200 \\
\hline $\mathrm{Cl}$ & -3.27262200 & -4.20895600 & 1.37660200 \\
\hline Mg & -5.39561200 & -4.31081300 & 0.05019100 \\
\hline $\mathrm{Cl}$ & -0.22850900 & -2.21141400 & 1.34819300 \\
\hline Mg & -2.35153700 & -2.31298000 & 0.02181500 \\
\hline $\mathrm{Cl}$ & -3.37347100 & -0.44114000 & 1.35338600 \\
\hline Mg & -5.60271800 & -0.67619900 & 0.10207900 \\
\hline $\mathrm{Cl}$ & -7.72597500 & -0.77795500 & -1.22432800 \\
\hline $\mathrm{Cl}$ & -6.52367900 & -2.57174600 & 1.45725000 \\
\hline $\mathrm{Ti}$ & -2.64188400 & 1.56220100 & -0.12389800 \\
\hline $\mathrm{Cl}$ & -4.57059900 & 1.03448400 & -1.46217800 \\
\hline $\mathrm{Cl}$ & -0.30348100 & 1.72827300 & 0.79884800 \\
\hline $\mathrm{C}$ & 4.53710800 & 3.39173000 & -0.18932700 \\
\hline C & 3.58275300 & 3.51534200 & 1.03422600 \\
\hline C & 4.46825400 & 4.66574500 & -1.05189900 \\
\hline $\mathrm{H}$ & 4.19307900 & 2.54015800 & -0.80241300 \\
\hline C & 2.32001600 & 4.34568300 & 0.70415100 \\
\hline $\mathrm{H}$ & 4.09841600 & 3.98125700 & 1.89439500 \\
\hline $\mathrm{H}$ & 3.29581700 & 2.50262500 & 1.35327900 \\
\hline C & 3.05907400 & 4.82389700 & -1.67441000 \\
\hline $\mathrm{H}$ & 4.70007400 & 5.54013800 & -0.41829100 \\
\hline $\mathrm{H}$ & 5.22693400 & 4.66357600 & -1.85225700 \\
\hline C & 1.95644800 & 4.21867900 & -0.77774300 \\
\hline $\mathrm{H}$ & 2.49226700 & 5.41188100 & 0.93549000 \\
\hline $\mathrm{H}$ & 1.48220900 & 4.01695300 & 1.33805700 \\
\hline $\mathrm{H}$ & 3.03476000 & 4.33814800 & -2.66438800 \\
\hline $\mathrm{H}$ & 2.85692300 & 5.89428200 & -1.85006800 \\
\hline $\mathrm{H}$ & 1.82207300 & 3.14857100 & -1.01255100 \\
\hline $\mathrm{H}$ & 0.98613400 & 4.69937600 & -0.98692400 \\
\hline C & 7.32070600 & 4.03921500 & 1.24671300 \\
\hline $\mathrm{H}$ & 8.23568800 & 3.51068700 & 1.55887800 \\
\hline $\mathrm{H}$ & 7.59966100 & 4.87403300 & 0.58363600 \\
\hline $\mathrm{H}$ & 6.82721800 & 4.46355400 & 2.13542900 \\
\hline 0 & 6.05741500 & 1.39608500 & 1.25140500 \\
\hline 0 & 7.08578500 & 2.04987800 & -0.89182300 \\
\hline C & 6.05280100 & 1.22002700 & 2.67151400 \\
\hline $\mathrm{H}$ & 5.23354500 & 0.53384000 & 2.92611400 \\
\hline $\mathrm{H}$ & 7.02381100 & 0.79584600 & 2.96839700 \\
\hline $\mathrm{H}$ & 5.88802600 & 2.18007700 & 3.18592300 \\
\hline
\end{tabular}




\begin{tabular}{|c|c|c|c|}
\hline$c$ & 7.12986000 & 2.38671000 & -2.27370500 \\
\hline $\mathrm{H}$ & 6.14321200 & 2.23894000 & -2.74148900 \\
\hline H & 7.46374700 & 3.42792100 & -2.41533200 \\
\hline-1 & 7.85345300 & 1.71591700 & -2.75833100 \\
\hline Si & 6.22826400 & 2.82254300 & 0.36312800 \\
\hline C & -3.25037600 & 2.97651000 & 1.24182800 \\
\hline $\mathrm{H}$ & -4.14248000 & 3.25837800 & 0.63852300 \\
\hline H & -3.63543500 & 2.37378800 & 2.08523900 \\
\hline C & -2.44908200 & 4.19484300 & 1.72923300 \\
\hline C & -1.83811000 & 3.93055100 & 3.11032500 \\
\hline C & -3.31140400 & 5.46617300 & 1.73821400 \\
\hline $\mathrm{H}$ & -1.60445600 & 4.37089400 & 1.03591600 \\
\hline $\mathrm{H}$ & -1.19842400 & 4.76999100 & 3.42809500 \\
\hline$H$ & -1.22418200 & 3.01824100 & 3.10499100 \\
\hline $\mathrm{H}$ & -2.63243500 & 3.80419700 & 3.86579600 \\
\hline $\mathrm{H}$ & -2.73185600 & 6.34103900 & 2.07733800 \\
\hline H & -4.17318200 & 5.34517100 & 2.41561200 \\
\hline H & -3.70938600 & 5.69303200 & 0.73462900 \\
\hline C & -1.66543400 & 2.87532600 & -2.15625300 \\
\hline H & -1.91816000 & 2.17095300 & -2.95568200 \\
\hline $\mathrm{H}$ & -0.65033800 & 2.84052600 & -1.75398600 \\
\hline C & -2.53097600 & 3.84056600 & -1.77178100 \\
\hline $\mathrm{H}$ & -2.20070900 & 4.53941800 & -0.99403100 \\
\hline C & -3.87282700 & 4.12081200 & -2.37062400 \\
\hline $\mathrm{H}$ & -4.65957700 & 4.15030300 & -1.59997300 \\
\hline $\mathrm{H}$ & -3.84972800 & 5.12005600 & -2.83963800 \\
\hline $\mathrm{H}$ & -4.15872300 & 3.37680600 & -3.12553100 \\
\hline C & -7.84961500 & 2.70922200 & -1.35139900 \\
\hline $\mathrm{H}$ & -6.86338700 & 3.05874900 & -0.99355300 \\
\hline $\mathrm{H}$ & -8.54773300 & 3.54678200 & -1.16049700 \\
\hline C & -10.06001400 & 0.84633800 & 0.71809600 \\
\hline $\mathrm{H}$ & -10.34957700 & 1.74741300 & 1.29264800 \\
\hline $\mathrm{H}$ & -10.83815800 & 0.74252700 & -0.06320700 \\
\hline C & -10.06380100 & -0.38894600 & 1.63579500 \\
\hline $\mathrm{H}$ & -11.04958300 & -0.56881800 & 2.10010500 \\
\hline $\mathrm{H}$ & -9.78707000 & -1.30238400 & 1.08296700 \\
\hline $\mathrm{H}$ & -9.32960600 & -0.28380300 & 2.45175400 \\
\hline C & -7.77276600 & 2.42396400 & -2.86098900 \\
\hline $\mathrm{H}$ & -8.74792900 & 2.10966800 & -3.271148 \\
\hline $\mathrm{H}$ & -7.45196700 & 3.30940700 & $-3.439324 €$ \\
\hline H & -7.05873500 & 1.61375300 & -3.07912900 \\
\hline $\mathrm{Cl}$ & -6.69122500 & 1.08002800 & $1.453757 €$ \\
\hline Al & -8.34622100 & 1.20184500 & $-0.17540 s$ \\
\hline
\end{tabular}


$\mathrm{MgCl}_{2}$-Ti-isobutyl-CMDMS-Mg2-AlEt ${ }_{2} \mathrm{Cl}$-Propylene-1, 2-re-TS

\begin{tabular}{|c|c|c|c|}
\hline $\mathrm{Cl}$ & 8.14136300 & -1.64757400 & -1.50931100 \\
\hline Mg & 7.22352200 & -3.56087600 & -0.17706500 \\
\hline 1 & 9.12543300 & 0.16595400 & 1.24637900 \\
\hline Mg & 7.00535000 & 0.07240600 & -0.08557500 \\
\hline $\mathrm{cl}$ & 5.10334400 & -3.65444200 & -1.50884600 \\
\hline $\mathrm{Cl}$ & 1.84710200 & -2.02796500 & -1.41681800 \\
\hline $\mathrm{Cl}$ & 4.88508400 & -0.02113500 & -1.41718500 \\
\hline $\mathrm{Cl}$ & 3.04946300 & -3.84769400 & 1.24721800 \\
\hline Mg & 0.92929300 & -3.94126300 & -0.08455300 \\
\hline $\mathrm{Cl}$ & 6.08747000 & -1.84081700 & $1.2468250 e$ \\
\hline $\mathrm{Mg}$ & 3.96731900 & -1.93439000 & -0.08505900 \\
\hline $\mathrm{Cl}$ & 2.83124700 & -0.21437900 & 1.33880900 \\
\hline Mg & 0.71090100 & -0.30836500 & 0.00705106 \\
\hline $\mathrm{Cl}$ & -1.19083900 & -4.03478800 & -1.4163460 \\
\hline $\mathrm{Cl}$ & -4.44713900 & -2.40835000 & -1.3243450 \\
\hline $\mathrm{Cl}$ & -1.31485500 & -0.46871000 & -1.5122140 \\
\hline $\mathrm{Cl}$ & -3.24486600 & -4.22808000 & 1.33964506 \\
\hline Mg & -5.36491900 & -4.32164000 & 0.00795506 \\
\hline $\mathrm{Cl}$ & -0.20673500 & -2.22125400 & 1.33936700 \\
\hline Mg & -2.32674100 & -2.31437900 & 0.00757500 \\
\hline $\mathrm{Cl}$ & -3.32942800 & -0.45356100 & 1.32234500 \\
\hline Mg & -5.58327800 & -0.68838100 & 0.09941700 \\
\hline $\mathrm{Cl}$ & -7.70338900 & -0.78194400 & -1.23219100 \\
\hline $\mathrm{Cl}$ & -6.50096700 & -2.60161500 & 1.43180006 \\
\hline $\mathrm{Ti}$ & -2.52242000 & 1.51990800 & -0.31020900 \\
\hline $\mathrm{Cl}$ & -4.62683300 & 1.05578800 & -1.45774400 \\
\hline $\mathrm{Cl}$ & -0.36329400 & 1.67847200 & 0.91900000 \\
\hline C & 4.50671900 & 3.37578000 & -0.14598600 \\
\hline C & 3.55172000 & 3.50811900 & 1.07548100 \\
\hline C & 4.40432300 & 4.62514100 & -1.04058600 \\
\hline $\mathrm{H}$ & 4.17842600 & 2.50363100 & -0.73758100 \\
\hline C & 2.26405400 & 4.29006700 & 0.72170700 \\
\hline $\mathrm{H}$ & 4.05452000 & 4.01506400 & 1.91981900 \\
\hline $\mathrm{H}$ & 3.29334400 & 2.49754500 & 1.42467000 \\
\hline C & 2.99131400 & 4.72531300 & -1.66748700 \\
\hline $\mathrm{H}$ & 4.61057100 & 5.52232000 & -0.43041700 \\
\hline $\mathrm{H}$ & 5.16260300 & 4.62341400 & -1.84106500 \\
\hline C & 1.90585200 & 4.11104100 & -0.75609300 \\
\hline $\mathrm{H}$ & 2.40342900 & 5.36715200 & 0.92279700 \\
\hline $\mathrm{H}$ & 1.43639600 & 3.95350700 & 1.36486200 \\
\hline $\mathrm{H}$ & 2.98235000 & 4.21374800 & -2.64451500 \\
\hline $\mathrm{H}$ & 2.75703900 & 5.78426900 & -1.87041700 \\
\hline & 1.80421000 & 3.03120600 & $-0.962334 \ell$ \\
\hline
\end{tabular}




\begin{tabular}{|c|c|c|c|}
\hline $\mathrm{H}$ & 0.92122900 & 4.55491200 & -0.98011200 \\
\hline C & 7. 27496100 & 4.07934200 & 1.29620100 \\
\hline $\mathrm{H}$ & 8.19992300 & 3.57106000 & 1.61221700 \\
\hline $\mathrm{H}$ & 7.53897200 & 4.91922400 & 0.63328400 \\
\hline $\mathrm{H}$ & 6.76977800 & 4.49455000 & 2.18265100 \\
\hline 0 & 6.06385200 & 1.40936300 & 1.29115800 \\
\hline 0 & 7.08709900 & 2.08749300 & -0.84414700 \\
\hline C & 6.06041900 & 1.22504900 & 2.71013900 \\
\hline $\mathrm{H}$ & 5.24548100 & 0.53236100 & 2.96130500 \\
\hline $\mathrm{H}$ & 7.03398000 & 0.80478800 & 3.00426200 \\
\hline $\mathrm{H}$ & 5.89024700 & 2.18115900 & 3.23024600 \\
\hline C & 7.14931900 & 2.43462700 & -2.22269200 \\
\hline $\mathrm{H}$ & 6.17452700 & 2.26938700 & -2.70902400 \\
\hline $\mathrm{H}$ & 7.46371900 & 3.48349400 & -2.35195900 \\
\hline $\mathrm{H}$ & 7.89552000 & 1.78229700 & -2.69821000 \\
\hline Si & 6.20975200 & 2.84188900 & 0.40808900 \\
\hline C & -3.33443800 & 3.29620900 & 0.60096100 \\
\hline $\mathrm{H}$ & -4.32493600 & 3.66313100 & 0.29920200 \\
\hline $\mathrm{H}$ & -3.57501800 & 2.31545700 & 1.11671700 \\
\hline C & -2.63456600 & 4.22121200 & 1.60116400 \\
\hline C & -2.90835800 & 3.74830200 & 3.03916400 \\
\hline C & -3.04889400 & 5.68568700 & 1.41278500 \\
\hline $\mathrm{H}$ & -1.54447700 & 4.14472200 & 1.44171600 \\
\hline $\mathrm{H}$ & -2.41697600 & 4.40981000 & 3.77083100 \\
\hline $\mathrm{H}$ & -2.52501200 & 2.72743500 & 3.19499700 \\
\hline $\mathrm{H}$ & -3.99001100 & 3.74361600 & 3.25468600 \\
\hline $\mathrm{H}$ & -2.52151700 & 6.33659500 & 2.12787900 \\
\hline $\mathrm{H}$ & -4.13236800 & 5.80911200 & 1.57999800 \\
\hline $\mathrm{H}$ & -2.82312500 & 6.05728200 & 0.40091500 \\
\hline C & -1.75056800 & 2.69340600 & -2.00820600 \\
\hline $\mathrm{H}$ & -2.19430500 & 2.21433400 & -2.88724200 \\
\hline $\mathrm{H}$ & -0.67176000 & 2.57386000 & -1.87326900 \\
\hline C & -2.38742800 & 3.79872500 & -1.42625800 \\
\hline $\mathrm{H}$ & -1.75669300 & 4.46764800 & -0.83476600 \\
\hline C & -3.60546500 & 4.41560000 & -2.06335700 \\
\hline $\mathrm{H}$ & -4.13102500 & 5.11125600 & -1.39649200 \\
\hline $\mathrm{H}$ & -3.26916600 & 4.98342700 & -2.94813400 \\
\hline $\mathrm{H}$ & -4.31149400 & 3.64390300 & -2.40026000 \\
\hline C & -7.94926400 & 2.70035600 & -1.24542300 \\
\hline $\mathrm{H}$ & -6.95649500 & 3.06739900 & -0.92617500 \\
\hline $\mathrm{H}$ & -8.66118400 & 3.51069300 & -0.99578500 \\
\hline C & -10.03992600 & 0.70181000 & 0.81118600 \\
\hline H & -10.34482200 & 1.57242800 & 1.42361206 \\
\hline $\mathrm{H}$ & -10.82963700 & 0.60296500 & 0.0407900 \\
\hline
\end{tabular}




$\begin{array}{lrrr}\text { C } & -9.99024100 & -0.56536600 & 1.68260300 \\ \text { H } & -10.96148600 & -0.79230300 & 2.15706100 \\ \text { H } & -9.69579000 & -1.44893700 & 1.09181700 \\ \text { H } & -9.24443100 & -0.46790500 & 2.48893200 \\ \text { C } & -7.93807300 & 2.46107200 & -2.76532800 \\ \text { H } & -8.91886000 & 2.11666800 & -3.13661000 \\ \text { H } & -7.68422300 & 3.37525200 & -3.33200400 \\ \text { H } & -7.20222300 & 1.68893100 & -3.04176700 \\ \text { Cl } & -6.67188200 & 1.03417000 & 1.49953500 \\ \text { Al } & -8.35699800 & 1.14209700 & -0.10456700\end{array}$

$\mathrm{MgCl}_{2}$-Ti-isobutyl-CMDMS-Mg2-AlEt ${ }_{2} \mathrm{Cl}$-Propylene-1, 2-si-coordination

$\begin{array}{lrrr}\text { Cl } & 8.19618600 & -1.69213800 & -1.41044100 \\ \text { Mg } & 7.23344000 & -3.61261400 & -0.12090700 \\ \text { Cl } & 9.15152000 & 0.07258300 & 1.38675200 \\ \text { Mg } & 7.05451900 & 0.02137100 & 0.01656400 \\ \text { Cl } & 5.13631000 & -3.66384900 & -1.49093100 \\ \text { Cl } & 1.89743200 & -2.00152100 & -1.43388400 \\ \text { Cl } & 4.95726800 & -0.02985300 & -1.35329700 \\ \text { Cl } & 3.03181200 & -3.87076600 & 1.22567300 \\ \text { Mg } & 0.93475500 & -3.92201200 & -0.14427600 \\ \text { Cl } & 6.09168000 & -1.89904100 & 1.30623300 \\ \text { Mg } & 3.99460500 & -1.95024400 & -0.06391500 \\ \text { Cl } & 2.85277700 & -0.23675900 & 1.36329300 \\ \text { Mg } & 0.75504700 & -0.28817200 & -0.00690800 \\ \text { Cl } & -1.16239300 & -3.97319300 & -1.51438000 \\ \text { Cl } & -4.40124600 & -2.31082800 & -1.45738900 \\ \text { Cl } & -1.30983500 & -0.38947200 & -1.49475800 \\ \text { Cl } & -3.26706700 & -4.18014600 & 1.20214500 \\ \text { Mg } & -5.36420400 & -4.23138000 & -0.16788300 \\ \text { Cl } & -0.20706200 & -2.20844500 & 1.28284300 \\ \text { Mg } & -2.30415700 & -2.25999700 & -0.08709100 \\ \text { Cl } & -3.33288300 & -0.38256500 & 1.28455100 \\ \text { Mg } & -5.54238700 & -0.59672200 & -0.03034000 \\ \text { Cl } & -7.64042200 & -0.64903900 & -1.40010500 \\ \text { Cl } & -6.50585000 & -2.51786200 & 1.25926500 \\ \text { Ti } & -2.50077400 & 1.66131800 & -0.10100200 \\ \text { Cl } & -4.54213500 & 1.31025800 & -1.35380100 \\ \text { Cl } & -0.06527800 & 1.86688400 & 0.61677400 \\ \text { C } & 4.76183700 & 3.44996600 & -0.20206300 \\ \text { C } & 3.71022600 & 3.57626700 & 0.93752100 \\ \text { C } & 4.81708700 & 4.74592400 & -1.03243500 \\ \text { H } & 4.44038100 & 2.62784600 & -0.86527100 \\ \text { C } & 2.52584300 & 4.48268300 & 0.52930600\end{array}$




\begin{tabular}{|c|c|c|c|}
\hline $\mathrm{H}$ & 4.16609200 & 3.98290800 & 1.85938300 \\
\hline $\mathrm{H}$ & 3.34845000 & 2.56778400 & 1.18967900 \\
\hline C & 3.47712200 & 4.97151900 & -1.77208400 \\
\hline $\mathrm{H}$ & 5.02368100 & 5.59546100 & -0.35767800 \\
\hline $\mathrm{H}$ & 5.64394900 & 4.73370000 & -1.76229200 \\
\hline C & 2.27651800 & 4.40665800 & -0.98011400 \\
\hline $\mathrm{H}$ & 2.73665000 & 5.53231700 & 0.80133900 \\
\hline $\mathrm{H}$ & 1.62434200 & 4.18766900 & 1.08784700 \\
\hline $\mathrm{H}$ & 3.51808200 & 4.49513400 & -2.76603800 \\
\hline $\mathrm{H}$ & 3.33726500 & 6.05116700 & -1.95182100 \\
\hline $\mathrm{H}$ & 2.10272300 & 3.35100600 & -1.25237000 \\
\hline H & 1.35608500 & 4.94672000 & -1.25534200 \\
\hline C & 7.49103600 & 3.99330700 & 1.37721200 \\
\hline $\mathrm{H}$ & 8.37498300 & 3.43242400 & 1.72114900 \\
\hline $\mathrm{H}$ & 7.82244200 & 4.81881600 & 0.72686600 \\
\hline $\mathrm{H}$ & 6.98014200 & 4.43323500 & 2.24852300 \\
\hline 0 & 6.12467200 & 1.40434300 & 1.34109800 \\
\hline 0 & 7.26237200 & 2.00353200 & -0.76628500 \\
\hline C & 6.07794500 & 1.22499300 & 2.76030600 \\
\hline $\mathrm{H}$ & 5.24621200 & 0.54393400 & 2.98679600 \\
\hline $\mathrm{H}$ & 7.03653100 & 0.79225300 & 3.08461000 \\
\hline $\mathrm{H}$ & 5.90422300 & 2.18487000 & 3.27207200 \\
\hline C & 7.36915600 & 2.31873200 & -2.14977000 \\
\hline $\mathrm{H}$ & 6.38917400 & 2.23357600 & -2.64662500 \\
\hline $\mathrm{H}$ & 7.77841500 & 3.33250800 & -2.29212200 \\
\hline $\mathrm{H}$ & 8.05799800 & 1.59268200 & -2.60431600 \\
\hline Si & 6.39023200 & 2.81626500 & 0.45213100 \\
\hline C & -2.79673300 & 2.97881300 & 1.46928300 \\
\hline $\mathrm{H}$ & -2.20550400 & 3.90829300 & 1.41171400 \\
\hline $\mathrm{H}$ & -2.33174700 & 2.36140900 & 2.26407800 \\
\hline C & -4.28264100 & 3.21087800 & 1.77540300 \\
\hline C & -4.48682000 & 3.57277400 & 3.25605100 \\
\hline $\mathrm{H}$ & -5.55968400 & 3.68210300 & 3.48572800 \\
\hline $\mathrm{H}$ & -3.98292000 & 4.52324200 & 3.50225600 \\
\hline $\mathrm{H}$ & -4.07837900 & 2.79166600 & 3.91664000 \\
\hline C & -1.60619100 & 2.88687100 & -2.18194700 \\
\hline $\mathrm{H}$ & -2.04446400 & 2.09958200 & -2.80261100 \\
\hline $\mathrm{H}$ & -0.52415700 & 2.86250900 & -2.02740200 \\
\hline C & -2.35603300 & 3.92316200 & -1.74319300 \\
\hline $\mathrm{H}$ & -3.42566900 & 3.92542400 & -1.98396600 \\
\hline C & -1.81690000 & 5.13315900 & -1.04626500 \\
\hline $\mathrm{H}$ & -2.50025500 & 5.48486800 & -0.26174800 \\
\hline $\mathrm{H}$ & -0.82674800 & 4.94309100 & -0.60824000 \\
\hline $\mathrm{H}$ & -1.72002600 & 5.95323000 & -1.78003400 \\
\hline
\end{tabular}




$\begin{array}{lrrr}\text { H } & -4.82020100 & 2.26441700 & 1.61455800 \\ \text { C } & -4.93310700 & 4.25494400 & 0.86418600 \\ \text { H } & -4.83963900 & 3.97668900 & -0.19553700 \\ \text { H } & -6.00899500 & 4.34493400 & 1.07971700 \\ \text { H } & -4.47627600 & 5.24952000 & 1.00794400 \\ \text { C } & -8.24109300 & 2.72182400 & -1.05980100 \\ \text { H } & -7.15092900 & 2.90185100 & -1.06896800 \\ \text { H } & -8.67175600 & 3.55497700 & -0.47125700 \\ \text { C } & -10.20975500 & 0.35775600 & 0.68580900 \\ \text { H } & -10.57127800 & 1.09917700 & 1.42470100 \\ \text { H } & -10.97747000 & 0.35304900 & -0.11233100 \\ \text { C } & -10.11733100 & -1.03246100 & 1.33813800 \\ \text { H } & -11.08200800 & -1.36831700 & 1.75845600 \\ \text { H } & -9.78967300 & -1.79520400 & 0.61215600 \\ \text { H } & -9.37846800 & -1.04525100 & 2.15640300 \\ \text { C } & -8.78574600 & 2.75549500 & -2.49880200 \\ \text { H } & -9.88004900 & 2.61130200 & -2.53018100 \\ \text { H } & -8.57602400 & 3.71253000 & -3.00948800 \\ \text { H } & -8.33942200 & 1.95542300 & -3.11348500 \\ \text { Cl } & -6.88820300 & 0.87137400 & 1.52112700 \\ \text { Al } & -8.53299500 & 1.03737100 & -0.08288800\end{array}$

$\mathrm{MgCl}_{2}$-Ti-isobutyl-CMDMS-Mg2-AlEt ${ }_{2} \mathrm{Cl}_{\text {-Propylene-1, 2-si-TS }}$

$\begin{array}{lrrr}\text { Cl } & -8.20852400 & -1.60807000 & 1.41233000 \\ \text { Mg } & -7.26844000 & -3.55949300 & 0.15287900 \\ \text { Cl } & -9.15034800 & 0.12056900 & -1.41182400 \\ \text { Mg } & -7.05144300 & 0.06965200 & -0.04448300 \\ \text { Cl } & -5.16944200 & -3.61043300 & 1.52005000 \\ \text { Cl } & -1.91336800 & -1.98355800 & 1.43033200 \\ \text { Cl } & -4.95235500 & 0.01872700 & 1.32251600 \\ \text { Cl } & -3.07225700 & -3.88407400 & -1.19630700 \\ \text { Mg } & -0.97339100 & -3.93503200 & 0.17074200 \\ \text { Cl } & -6.11132200 & -1.88170300 & -1.30409200 \\ \text { Mg } & -4.01239800 & -1.93255700 & 0.06325200 \\ \text { Cl } & -2.85519400 & -0.25486000 & -1.39380300 \\ \text { Mg } & -0.75485000 & -0.30628800 & -0.02659500 \\ \text { Cl } & 1.12569000 & -3.98591800 & 1.53804400 \\ \text { Cl } & 4.38189700 & -2.35912300 & 1.44835100 \\ \text { Cl } & 1.27653700 & -0.39823000 & 1.48905100 \\ \text { Cl } & 3.22306400 & -4.25957200 & -1.17824900 \\ \text { Mg } & 5.32195600 & -4.31052300 & 0.18886400 \\ \text { Cl } & 0.18380400 & -2.25722800 & -1.28621700 \\ \text { Mg } & 2.28245700 & -2.30851100 & 0.08127900 \\ \text { Cl } & 3.31031300 & -0.50434300 & -1.31461200\end{array}$




\begin{tabular}{|c|c|c|c|}
\hline Mg & 5.53811900 & -0.68099500 & -0.00869500 \\
\hline $\mathrm{Cl}$ & 7.63799300 & -0.73233400 & 1.35852800 \\
\hline $\mathrm{Cl}$ & 6.47906600 & -2.63275400 & -1.26808300 \\
\hline $\mathrm{Ti}$ & 2.45830100 & 1.54666500 & 0.16295000 \\
\hline $\mathrm{Cl}$ & 4.59380000 & 1.18839600 & 1.34447100 \\
\hline $\mathrm{Cl}$ & 0.26148400 & 1.70523100 & -0.96369400 \\
\hline C & -4.76333600 & 3.46109800 & 0.20991200 \\
\hline C & -3.67503800 & 3.55267000 & -0.89457100 \\
\hline C & -4.78535700 & 4.74861600 & 1.05351700 \\
\hline $\mathrm{H}$ & -4.48846500 & 2.62274500 & 0.87323300 \\
\hline C & -2.43657800 & 4.34588600 & -0.41098900 \\
\hline $\mathrm{H}$ & -4.07289400 & 4.03283500 & -1.80774200 \\
\hline $\mathrm{H}$ & -3.38337500 & 2.53062700 & -1.18120300 \\
\hline C & -3.46114800 & 4.89366500 & 1.84282500 \\
\hline $\mathrm{H}$ & -4.92437800 & 5.61502200 & 0.38291400 \\
\hline $\mathrm{H}$ & -5.63756100 & 4.77024000 & 1.75333500 \\
\hline C & -2.27432000 & 4.23262500 & 1.10824700 \\
\hline $\mathrm{H}$ & -2.53864200 & 5.41335100 & -0.67604200 \\
\hline $\mathrm{H}$ & -1.53815300 & 3.97824700 & -0.93062600 \\
\hline $\mathrm{H}$ & -3.57158100 & 4.44034600 & 2.84217600 \\
\hline $\mathrm{H}$ & -3.24951700 & 5.96360900 & 2.00984200 \\
\hline $\mathrm{H}$ & -2.21194600 & 3.16314900 & 1.37767000 \\
\hline $\mathrm{H}$ & -1.32752900 & 4.68558900 & 1.44051500 \\
\hline C & -7.47697200 & 4.05406800 & -1.40009800 \\
\hline $\mathrm{H}$ & -8.36421200 & 3.50359200 & -1.75213200 \\
\hline $\mathrm{H}$ & -7.80449500 & 4.88096500 & -0.74956700 \\
\hline $\mathrm{H}$ & -6.95531800 & 4.49128600 & -2.26650000 \\
\hline 0 & -6.12659300 & 1.45409400 & -1.36581300 \\
\hline 0 & -7.27443000 & 2.04875200 & 0.73723000 \\
\hline C & -6.08015500 & 1.27241300 & -2.78472400 \\
\hline $\mathrm{H}$ & -5.25843500 & 0.57822400 & -3.00819600 \\
\hline $\mathrm{H}$ & -7.04422700 & 0.85314400 & -3.11061400 \\
\hline $\mathrm{H}$ & -5.88987800 & 2.22892200 & -3.29698100 \\
\hline C & -7.39467300 & 2.35850600 & 2.12091200 \\
\hline $\mathrm{H}$ & -6.42043100 & 2.26676800 & 2.62791000 \\
\hline $\mathrm{H}$ & -7.80066800 & 3.37352100 & 2.26357000 \\
\hline $\mathrm{H}$ & -8.09138200 & 1.63311300 & 2.56433500 \\
\hline Si & -6.39316700 & 2.86404300 & -0.47256900 \\
\hline C & 3.19527400 & 3.29342100 & -0.88971100 \\
\hline $\mathrm{H}$ & 2.45190700 & 3.89115100 & -1.43875100 \\
\hline $\mathrm{H}$ & 3.21949100 & 2.30128300 & -1.44261400 \\
\hline C & 4.60414300 & 3.87255400 & -1.02531900 \\
\hline C & 5.04827000 & 3.73953000 & -2.49561200 \\
\hline $\mathrm{H}$ & 6.08831300 & 4.08236300 & -2.61580000 \\
\hline
\end{tabular}




$\begin{array}{lrrr}\text { H } & 4.40697200 & 4.34920500 & -3.15499900 \\ \text { H } & 5.00812300 & 2.69544600 & -2.83861100 \\ \text { C } & 1.78420500 & 2.77127000 & 1.85675200 \\ \text { H } & 2.33981200 & 2.29560000 & 2.66964400 \\ \text { H } & 0.69373700 & 2.67425400 & 1.88387400 \\ \text { C } & 2.34948700 & 3.85845900 & 1.17247900 \\ \text { H } & 3.37110600 & 4.12609600 & 1.45023600 \\ \mathrm{C} & 1.47626700 & 4.96335500 & 0.63809500 \\ \text { H } & 2.01162300 & 5.64957700 & -0.02843300 \\ \text { H } & 0.60584500 & 4.55153100 & 0.10890600 \\ \text { H } & 1.10434200 & 5.54817500 & 1.49662200 \\ \text { H } & 5.28285700 & 3.25596700 & -0.41564500 \\ \mathrm{C} & 4.73505500 & 5.33568300 & -0.58446800 \\ \text { H } & 4.50770300 & 5.48933500 & 0.48016400 \\ \text { H } & 5.76809600 & 5.68118300 & -0.74497400 \\ \text { H } & 4.07238500 & 5.99163400 & -1.17470500 \\ \mathrm{C} & 8.19385800 & 2.66185600 & 1.04022300 \\ \mathrm{H} & 7.10531200 & 2.76427000 & 1.19944000 \\ \mathrm{H} & 8.48314200 & 3.51379800 & 0.39451600 \\ \mathrm{C} & 10.11358400 & 0.33339900 & -0.80172100 \\ \mathrm{H} & 10.48035400 & 1.11264200 & -1.49775900 \\ \mathrm{H} & 10.89139900 & 0.26241100 & -0.01652100 \\ \mathrm{C} & 9.98185300 & -1.01112100 & -1.53757700 \\ \mathrm{H} & 10.93619800 & -1.34829100 & -1.97991900 \\ \mathrm{H} & 9.63082000 & -1.80711200 & -0.86001500 \\ \mathrm{H} & 9.24285200 & -0.95158200 & -2.35367200 \\ \mathrm{C} & 8.92530200 & 2.74920200 & 2.39148700 \\ \mathrm{H} & 10.02105300 & 2.67934300 & 2.27441900 \\ \mathrm{H} & 8.72164100 & 3.69526500 & 2.92464900 \\ \mathrm{H} & 8.62102200 & 1.92877200 & 3.06377000 \\ \mathrm{Cl} & 6.77014900 & 0.86013100 & -1.53439200 \\ \mathrm{Al} & 8.46212600 & 0.98799400 & 0.04033400\end{array}$

$\mathrm{MgCl}_{2}$-Ti-isobutyl-CMDMS-Mg2-AlEt ${ }_{2} \mathrm{Cl}$-Propylene-2,1-re-coordination

$\begin{array}{lrrr}\text { Cl } & -8.03308800 & -1.84397000 & 1.50251900 \\ \text { Mg } & -7.07753100 & -3.70072700 & 0.11781200 \\ \text { Cl } & -9.06111300 & 0.02175200 & -1.20176000 \\ \text { Mg } & -6.93674500 & -0.06248300 & 0.12403100 \\ \text { Cl } & -4.95310800 & -3.78497000 & 1.44342700 \\ \text { Cl } & -1.73248500 & -2.08762900 & 1.39037400 \\ \text { Cl } & -4.81225700 & -0.14668700 & 1.44946300 \\ \text { Cl } & -2.90126900 & -3.86015800 & -1.31990000 \\ \text { Mg } & -0.77686300 & -3.94440200 & 0.00569900 \\ \text { Cl } & -5.98121100 & -1.91915100 & -1.26085600\end{array}$




\begin{tabular}{|c|c|c|c|}
\hline Mg & -3.85680900 & -2.00339700 & 0.06485700 \\
\hline $\mathrm{Cl}$ & -2.76045900 & -0.22191400 & -1.31380400 \\
\hline Mg & -0.63529300 & -0.30630600 & 0.01203300 \\
\hline $\mathrm{Cl}$ & 1.34749200 & -4.02856500 & 1.33136400 \\
\hline $\mathrm{Cl}$ & 4.56836300 & -2.33125000 & 1.27848000 \\
\hline $\mathrm{Cl}$ & 1.43722600 & -0.47870200 & 1.48348100 \\
\hline $\mathrm{Cl}$ & 3.39954800 & -4.10381300 & -1.43192600 \\
\hline Mg & 5.52384100 & -4.18808500 & -0.10635800 \\
\hline $\mathrm{Cl}$ & 0.31947200 & -2.16281400 & -1.37300400 \\
\hline Mg & 2.44381600 & -2.24724100 & -0.04748200 \\
\hline $\mathrm{Cl}$ & 3.41914800 & -0.31620300 & -1.33466100 \\
\hline Mg & 5.66406000 & -0.54975700 & -0.10037700 \\
\hline $\mathrm{Cl}$ & 7.78913800 & -0.63408400 & 1.22534300 \\
\hline $\mathrm{Cl}$ & 6.62025100 & -2.40648300 & -1.48499900 \\
\hline $\mathrm{Ti}$ & 2.63373400 & 1.62206900 & 0.20990400 \\
\hline $\mathrm{Cl}$ & 4.62704300 & 1.13786100 & 1.49215400 \\
\hline $\mathrm{Cl}$ & 0.24723100 & 1.84814400 & -0.56015400 \\
\hline C & -4.63265200 & 3.41474300 & 0.08005500 \\
\hline C & -3.72392000 & 3.52144600 & -1.18100300 \\
\hline C & -4.63207200 & 4.74392200 & 0.85802900 \\
\hline $\mathrm{H}$ & -4.22106600 & 2.62263500 & 0.73213700 \\
\hline C & -2.51892600 & 4.46436700 & -0.95475600 \\
\hline $\mathrm{H}$ & -4.29790900 & 3.88400100 & -2.05393100 \\
\hline $\mathrm{H}$ & -3.37094800 & 2.51200000 & -1.43862800 \\
\hline C & -3.22032600 & 5.05799700 & 1.41026300 \\
\hline $\mathrm{H}$ & -4.95569400 & 5.55281200 & 0.17942000 \\
\hline $\mathrm{H}$ & -5.36139300 & 4.73552300 & 1.68542700 \\
\hline C & -2.10753100 & 4.46795300 & 0.51885700 \\
\hline $\mathrm{H}$ & -2.77569600 & 5.49503400 & -1.25756800 \\
\hline $\mathrm{H}$ & -1.67576900 & 4.15089100 & -1.59003700 \\
\hline $\mathrm{H}$ & -3.12156100 & 4.65591100 & 2.43305300 \\
\hline $\mathrm{H}$ & -3.09761500 & 6.15119300 & 1.49568700 \\
\hline $\mathrm{H}$ & -1.90063700 & 3.42686400 & 0.81421400 \\
\hline $\mathrm{H}$ & -1.16406200 & 5.02038700 & 0.66368000 \\
\hline C & -7.49165400 & 3.84813100 & -1.28759500 \\
\hline $\mathrm{H}$ & -8.37070700 & 3.24313200 & -1.56385100 \\
\hline $\mathrm{H}$ & -7.81637500 & 4.67401700 & -0.63436000 \\
\hline $\mathrm{H}$ & -7.05205600 & 4.28726200 & -2.19701000 \\
\hline 0 & -6.05394000 & 1.29732100 & -1.26977500 \\
\hline 0 & -7.07219400 & 1.93706700 & 0.88836400 \\
\hline C & -6.05137500 & 1.10831500 & -2.68845300 \\
\hline $\mathrm{H}$ & -5.21623700 & 0.44062200 & -2.93994300 \\
\hline $\mathrm{H}$ & -7.01274800 & 0.65813100 & -2.97786200 \\
\hline \multirow[t]{2}{*}{$\mathrm{H}$} & -5.91184000 & 2.06745000 & -3.21170600 \\
\hline & & & S114 \\
\hline
\end{tabular}




\begin{tabular}{|c|c|c|c|}
\hline C & -7.07343600 & 2.29736500 & 2.26522100 \\
\hline $\mathrm{H}$ & -6.06124000 & 2.20925600 & 2.69161300 \\
\hline $\mathrm{H}$ & -7.45444600 & 3.32277300 & 2.40308600 \\
\hline-1 & -7.73941200 & 1.60046000 & 2.79343100 \\
\hline $\mathrm{Si}$ & -6.29838600 & 2.72825600 & -0.40837900 \\
\hline C & 3.12508600 & 3.07320000 & -1.15594300 \\
\hline $\mathrm{H}$ & 4.09420500 & 3.27362500 & -0.64289700 \\
\hline H & 3.37403000 & 2.50829300 & -2.07336900 \\
\hline C & 2.32704600 & 4.35160000 & -1.46175300 \\
\hline C & 1.61210400 & 4.23881500 & -2.81327200 \\
\hline C & 3.21878400 & 5.60021200 & -1.40175700 \\
\hline $\mathrm{H}$ & 1.53784700 & 4.47365000 & -0.69641400 \\
\hline $\mathrm{H}$ & 0.96933300 & 5.11533500 & -2.99671500 \\
\hline $\mathrm{H}$ & 0.97996800 & 3.33940200 & -2.85191100 \\
\hline $\mathrm{H}$ & 2.34522200 & 4.17720700 & -3.63569000 \\
\hline $\mathrm{H}$ & 2.63859900 & 6.51569100 & -1.60601500 \\
\hline H & 4.02814800 & 5.53686100 & -2.14823700 \\
\hline $\mathrm{H}$ & 3.68855300 & 5.71151300 & -0.41015600 \\
\hline C & 1.75449500 & 2.63524400 & 2.68677500 \\
\hline $\mathrm{H}$ & 2.36914100 & 1.90927900 & 3.23396700 \\
\hline C & 2.38752500 & 3.57644700 & 1.95201500 \\
\hline $\mathrm{H}$ & 1.82148200 & 4.33933800 & 1.40938100 \\
\hline $\mathrm{H}$ & 3.47635300 & 3.67062500 & 1.96679100 \\
\hline C & 0.27643900 & 2.54448500 & 2.89221200 \\
\hline $\mathrm{H}$ & -0.08251200 & 1.51473100 & 2.74583500 \\
\hline $\mathrm{H}$ & 0.04550200 & 2.81057400 & 3.93861500 \\
\hline $\mathrm{H}$ & -0.27573000 & 3.21806000 & 2.22567400 \\
\hline C & 8.17026100 & 2.78735200 & 1.28635300 \\
\hline H & 7.07427200 & 2.89762900 & 1.37753200 \\
\hline $\mathrm{H}$ & 8.52282300 & 3.71448800 & 0.79468200 \\
\hline C & 10.11916200 & 0.77889500 & -0.89089500 \\
\hline $\mathrm{H}$ & 10.42769800 & 1.64114600 & -1.51311400 \\
\hline H & 10.92801000 & 0.66154700 & -0.14331800 \\
\hline C & 10.01442700 & -0.48712400 & -1.75869200 \\
\hline $\mathrm{H}$ & 10.96641500 & -0.73738600 & -2.25973000 \\
\hline $\mathrm{H}$ & 9.71528000 & -1.36302600 & -1.15917200 \\
\hline $\mathrm{H}$ & 9.24942000 & -0.37301600 & -2.54460800 \\
\hline C & 8.79679300 & 2.66134000 & 2.68661100 \\
\hline $\mathrm{H}$ & 9.89725200 & 2.58183600 & 2.64204600 \\
\hline $\mathrm{H}$ & 8.56614000 & 3.52721500 & 3.33282700 \\
\hline H & 8.43067500 & 1.76065400 & 3.20784500 \\
\hline $\mathrm{Cl}$ & 6.72586900 & 1.23414900 & -1.4426906 \\
\hline Al & 8.47939300 & 1.26653900 & 0.076565 \\
\hline
\end{tabular}




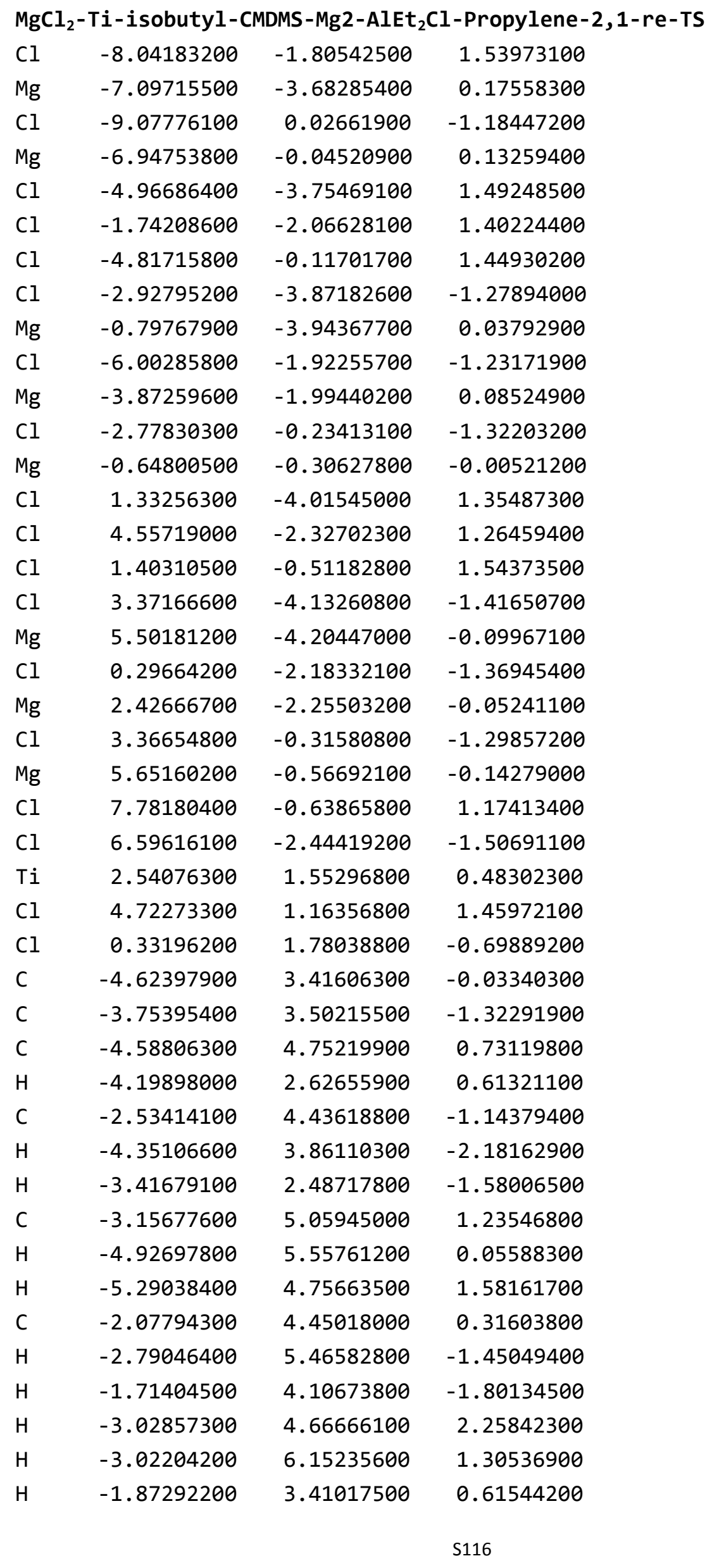




\begin{tabular}{|c|c|c|c|}
\hline $\mathrm{H}$ & -1.12448400 & 4.99299400 & 0.42775900 \\
\hline C & -7.51031100 & 3.83415500 & -1.35304200 \\
\hline $\mathrm{H}$ & -8.39249500 & 3.22253700 & -1.60376600 \\
\hline $\mathrm{H}$ & -7.82648400 & 4.67175200 & -0.71057500 \\
\hline $\mathrm{H}$ & -7.08576300 & 4.25628900 & -2.27746000 \\
\hline 0 & -6.07512500 & 1.28062100 & -1.30266600 \\
\hline 0 & -7.05943300 & 1.97157000 & 0.85597900 \\
\hline C & -6.08674100 & 1.06506200 & -2.71740900 \\
\hline $\mathrm{H}$ & -5.25423700 & 0.39265700 & -2.96519200 \\
\hline $\mathrm{H}$ & -7.05101700 & 0.61007900 & -2.98889800 \\
\hline $\mathrm{H}$ & -5.95257900 & 2.01435200 & -3.25972600 \\
\hline C & -7.03818600 & 2.36588400 & 2.22363000 \\
\hline $\mathrm{H}$ & -6.02121300 & 2.27718500 & 2.63810400 \\
\hline $\mathrm{H}$ & -7.40624200 & 3.39858500 & 2.34086800 \\
\hline $\mathrm{H}$ & -7.70420200 & 1.68980100 & 2.77811700 \\
\hline Si & -6.30438800 & 2.73082400 & -0.47025700 \\
\hline C & 3.16205800 & 3.43594400 & -0.35429100 \\
\hline $\mathrm{H}$ & 4.16783600 & 3.80478200 & -0.10681100 \\
\hline $\mathrm{H}$ & 3.37467800 & 2.52773800 & -1.00776300 \\
\hline C & 2.33539300 & 4.45103500 & -1.15152300 \\
\hline C & 2.37424100 & 4.12609700 & -2.65380100 \\
\hline C & 2.80523800 & 5.88664900 & -0.88580400 \\
\hline $\mathrm{H}$ & 1.28296600 & 4.36204100 & -0.83121400 \\
\hline $\mathrm{H}$ & 1.77713500 & 4.85444700 & -3.22594100 \\
\hline $\mathrm{H}$ & 1.96227200 & 3.12397600 & -2.84942200 \\
\hline $\mathrm{H}$ & 3.40736200 & 4.15683300 & -3.03883000 \\
\hline $\mathrm{H}$ & 2.16433600 & 6.61210600 & -1.41148200 \\
\hline $\mathrm{H}$ & 3.83955100 & 6.02979500 & -1.24166700 \\
\hline $\mathrm{H}$ & 2.78598200 & 6.13994600 & 0.18623900 \\
\hline C & 1.83922200 & 2.55973000 & 2.38132100 \\
\hline $\mathrm{H}$ & 2.42963500 & 1.92262800 & 3.05308400 \\
\hline C & 2.51334100 & 3.63608500 & 1.80137400 \\
\hline $\mathrm{H}$ & 1.93896400 & 4.49937400 & 1.45944400 \\
\hline $\mathrm{H}$ & 3.55443100 & 3.82658400 & 2.06561700 \\
\hline C & 0.34530500 & 2.57079400 & 2.61661300 \\
\hline $\mathrm{H}$ & -0.07983700 & 1.55628700 & 2.61724400 \\
\hline $\mathrm{H}$ & 0.12555000 & 3.00994900 & 3.60550200 \\
\hline $\mathrm{H}$ & -0.17707500 & 3.16289900 & 1.85593200 \\
\hline C & 8.19887700 & 2.78582600 & 1.14357100 \\
\hline $\mathrm{H}$ & 7.10567300 & 2.93281700 & 1.21085400 \\
\hline $\mathrm{H}$ & 8.59254400 & 3.68804000 & 0.63669000 \\
\hline C & 10.11018000 & 0.70299900 & -0.98997400 \\
\hline $\mathrm{H}$ & 10.41967800 & 1.54757900 & -1.63562700 \\
\hline $\mathrm{H}$ & 10.92338300 & 0.59951800 & -0.24502000 \\
\hline
\end{tabular}




$\begin{array}{lrrr}\text { C } & 9.99243400 & -0.58362900 & -1.82497300 \\ \text { H } & 10.93944900 & -0.85228100 & -2.32608000 \\ \text { H } & 9.69216400 & -1.44211000 & -1.20140100 \\ \text { H } & 9.22243100 & -0.48503400 & -2.60807900 \\ \text { C } & 8.79297300 & 2.67392100 & 2.55900200 \\ \text { H } & 9.89066000 & 2.55510200 & 2.53930400 \\ \text { H } & 8.58023100 & 3.56336300 & 3.17898400 \\ \text { H } & 8.38482900 & 1.79987300 & 3.09389800 \\ \text { Cl } & 6.71954700 & 1.17980800 & -1.53628200 \\ \text { Al } & 8.48000800 & 1.22577200 & -0.02362300\end{array}$

$\mathrm{MgCl}_{2}$-Ti-isobutyl-CMDMS-Mg2-AlEt ${ }_{2} \mathrm{Cl}$-Propylene-2,1-si-coordination

$\begin{array}{lrrr}\text { Cl } & -8.10289300 & -1.76590300 & 1.42936500 \\ \text { Mg } & -7.12597000 & -3.63530700 & 0.07696300 \\ \text { Cl } & -9.09508700 & 0.07116400 & -1.30773100 \\ \text { Mg } & -6.98941200 & 0.00299400 & 0.04841200 \\ \text { Cl } & -5.02020900 & -3.70348600 & 1.43293500 \\ \text { Cl } & -1.80135400 & -2.00265700 & 1.40759000 \\ \text { Cl } & -4.88356000 & -0.06515700 & 1.40420400 \\ \text { Cl } & -2.92981700 & -3.80391800 & -1.30053100 \\ \text { Mg } & -0.82408400 & -3.87210600 & 0.05540800 \\ \text { Cl } & -6.01246200 & -1.86632700 & -1.30415000 \\ \text { Mg } & -3.90674400 & -1.93449500 & 0.05192500 \\ \text { Cl } & -2.79321000 & -0.16564500 & -1.32920200 \\ \text { Mg } & -0.68599900 & -0.23393000 & 0.02738600 \\ \text { Cl } & 1.28159900 & -3.94016900 & 1.41147100 \\ \text { Cl } & 4.50096600 & -2.23930400 & 1.38656000 \\ \text { Cl } & 1.36916300 & -0.36131200 & 1.50658200 \\ \text { Cl } & 3.37224800 & -4.04068100 & -1.32197400 \\ \text { Mg } & 5.47786900 & -4.10890800 & 0.03396800 \\ \text { Cl } & 0.28941800 & -2.10310900 & -1.32574300 \\ \text { Mg } & 2.39515800 & -2.17169500 & 0.03000500 \\ \text { Cl } & 3.39803100 & -0.29843100 & -1.29061600 \\ \text { Mg } & 5.61333400 & -0.47050400 & 0.00490000 \\ \text { Cl } & 7.72028200 & -0.53875400 & 1.36129000 \\ \text { Cl } & 6.59150100 & -2.33987100 & -1.34713200 \\ \text { Ti } & 2.59721600 & 1.70508800 & 0.12326000 \\ \text { Cl } & 4.55844800 & 1.31592600 & 1.47333800 \\ \text { Cl } & 0.20875400 & 1.90774300 & -0.63852800 \\ \text { C } & -4.65630800 & 3.41372700 & 0.25829500 \\ \text { C } & -3.65768200 & 3.58029400 & -0.92366200 \\ \text { C } & -4.65796900 & 4.67544900 & 1.14146600 \\ \text { H } & -4.31246000 & 2.56335000 & 0.87284400 \\ \text { C } & -2.44083100 & 4.45058500 & -0.53234000\end{array}$




\begin{tabular}{|c|c|c|c|}
\hline $\mathrm{H}$ & -4.15176700 & 4.03550800 & -1.80212500 \\
\hline $\mathrm{H}$ & -3.32296000 & 2.58047000 & -1.23803100 \\
\hline C & -3.28100800 & 4.85839100 & 1.82503800 \\
\hline $\mathrm{H}$ & -4.88552500 & 5.55353200 & 0.51153500 \\
\hline $\mathrm{H}$ & -5.44952000 & 4.64171300 & 1.90860400 \\
\hline C & -2.12590700 & 4.30690300 & 0.95954200 \\
\hline $\mathrm{H}$ & -2.64540400 & 5.51396300 & -0.74991700 \\
\hline $\mathrm{H}$ & -1.56975400 & 4.16663100 & -1.14226300 \\
\hline $\mathrm{H}$ & -3.28312000 & 4.34871800 & 2.80334300 \\
\hline $\mathrm{H}$ & -3.11769000 & 5.92930000 & 2.03446900 \\
\hline $\mathrm{H}$ & -1.96243800 & 3.23746200 & 1.17925400 \\
\hline $\mathrm{H}$ & -1.18118000 & 4.81557100 & 1.21415500 \\
\hline C & -7.41597500 & 4.00848800 & -1.24745900 \\
\hline $\mathrm{H}$ & -8.30958300 & 3.46135400 & -1.58828700 \\
\hline $\mathrm{H}$ & -7.73113300 & 4.82501500 & -0.57790000 \\
\hline $\mathrm{H}$ & -6.91451800 & 4.45991700 & -2.11826400 \\
\hline 0 & -6.07282400 & 1.40532500 & -1.27183900 \\
\hline 0 & -7.17258700 & 1.98333900 & 0.85896700 \\
\hline C & -6.04417000 & 1.25205300 & -2.69448500 \\
\hline $\mathrm{H}$ & -5.21272400 & 0.57940400 & -2.94566600 \\
\hline $\mathrm{H}$ & -7.00497600 & 0.82050500 & -3.01345800 \\
\hline $\mathrm{H}$ & -5.88214300 & 2.22196100 & -3.19088100 \\
\hline C & -7.26416400 & 2.28715900 & 2.24601700 \\
\hline H & -6.28494500 & 2.16543200 & 2.73657600 \\
\hline $\mathrm{H}$ & -7.64019100 & 3.31185500 & 2.40147900 \\
\hline $\mathrm{H}$ & -7.97418600 & 1.57909400 & 2.69649100 \\
\hline Si & -6.31031500 & 2.80738600 & -0.35942200 \\
\hline C & 3.27451400 & 3.02047800 & -1.30363700 \\
\hline $\mathrm{H}$ & 3.38626800 & 3.91370200 & -0.65116200 \\
\hline $\mathrm{H}$ & 4. 29871800 & 2.62581600 & -1.46645200 \\
\hline C & 2.59235900 & 3.38588200 & -2.62589300 \\
\hline C & 2.43049400 & 2.16861600 & -3.54324700 \\
\hline C & 3.36376600 & 4.50937000 & -3.33800000 \\
\hline $\mathrm{H}$ & 1.58242100 & 3.76538200 & -2.38694600 \\
\hline $\mathrm{H}$ & 1.96732300 & 2.45744400 & -4.50046400 \\
\hline $\mathrm{H}$ & 1.79727300 & 1.39906800 & -3.07881700 \\
\hline $\mathrm{H}$ & 3.40953300 & 1.71076300 & -3.76295000 \\
\hline H & 2.85700700 & 4.80956400 & -4.27046900 \\
\hline $\mathrm{H}$ & 4.38248500 & 4.17543300 & -3.59817100 \\
\hline $\mathrm{H}$ & 3.45757900 & 5.40299400 & -2.69947200 \\
\hline C & 1.47585100 & 2.81471000 & 2.56790100 \\
\hline C & 2.02700000 & 3.71712000 & 1.72999200 \\
\hline $\mathrm{H}$ & 1.43946800 & 4.14308400 & 0.91055800 \\
\hline $\mathrm{H}$ & 3.02817500 & 4.12203000 & 1.90918800 \\
\hline
\end{tabular}




$\begin{array}{lrrr}\text { H } & 0.45327900 & 2.48077100 & 2.35348200 \\ \text { C } & 2.12008800 & 2.25647200 & 3.79443700 \\ \text { H } & 1.55853100 & 2.59522700 & 4.68286100 \\ \text { H } & 2.07612500 & 1.15671200 & 3.78860100 \\ \text { H } & 3.16879200 & 2.56976300 & 3.88947600 \\ \text { C } & 8.13988900 & 2.87793400 & 1.28690100 \\ \text { H } & 7.04411600 & 3.01758200 & 1.32338900 \\ \text { H } & 8.53726800 & 3.77610200 & 0.77602900 \\ \text { C } & 10.12207800 & 0.75660400 & -0.75098300 \\ \text { H } & 10.46100200 & 1.59035700 & -1.39580500 \\ \text { H } & 10.90790000 & 0.65557100 & 0.02303700 \\ \text { C } & 10.02171500 & -0.53947100 & -1.57369600 \\ \text { H } & 10.98308500 & -0.82197800 & -2.03838500 \\ \text { H } & 9.69281300 & -1.38748900 & -0.95023300 \\ \text { H } & 9.27979200 & -0.44445000 & -2.38377800 \\ \text { C } & 8.69997400 & 2.79345600 & 2.71800200 \\ \text { H } & 9.79915800 & 2.68834200 & 2.72713100 \\ \text { H } & 8.46109100 & 3.68907200 & 3.31921600 \\ \text { H } & 8.29000100 & 1.92207400 & 3.25603500 \\ \text { Cl } & 6.75193500 & 1.22343300 & -1.41363900 \\ \text { Al } & 8.46385000 & 1.30411900 & 0.15100700\end{array}$

$\mathrm{MgCl}_{2}$-Ti-isobutyl-CMDMS-Mg2-AlEt ${ }_{2} \mathrm{Cl}_{\text {-Propylene-2,1-si-TS }}$

$\begin{array}{lrrr}\text { Cl } & -8.13591600 & -1.67923800 & 1.46805000 \\ \text { Mg } & -7.20070900 & -3.57838200 & 0.12765400 \\ \text { Cl } & -9.12299600 & 0.14390300 & -1.28021900 \\ \text { Mg } & -7.00681300 & 0.05677000 & 0.05834200 \\ \text { Cl } & -5.08441800 & -3.66553300 & 1.46604200 \\ \text { Cl } & -1.83941100 & -2.01653100 & 1.39440000 \\ \text { Cl } & -4.89042900 & -0.03036100 & 1.39655700 \\ \text { Cl } & -3.02005300 & -3.82860700 & -1.28413500 \\ \text { Mg } & -0.90376700 & -3.91575800 & 0.05425200 \\ \text { Cl } & -6.07154600 & -1.84229800 & -1.28220300 \\ \text { Mg } & -3.95530000 & -1.92943700 & 0.05630800 \\ \text { Cl } & -2.82609100 & -0.19349100 & -1.35358100 \\ \text { Mg } & -0.70871900 & -0.28102400 & -0.01460200 \\ \text { Cl } & 1.21243700 & -4.00282400 & 1.39267900 \\ \text { Cl } & 4.45794800 & -2.35387800 & 1.32137500 \\ \text { Cl } & 1.31024600 & -0.42616000 & 1.51415600 \\ \text { Cl } & 3.27700800 & -4.16599800 & -1.35743200 \\ \text { Mg } & 5.39318500 & -4.25315100 & -0.01911500 \\ \text { Cl } & 0.22536000 & -2.17975600 & -1.35561700 \\ \text { Mg } & 2.34154900 & -2.26674400 & -0.01768800 \\ \text { Cl } & 3.34053500 & -0.40151200 & -1.31715400\end{array}$




\begin{tabular}{|c|c|c|c|}
\hline $\mathrm{Mg}$ & 5.58662000 & -0.61793900 & -0.08866700 \\
\hline $\mathrm{Cl}$ & 7.70343200 & -0.70511600 & 1.24979200 \\
\hline $\mathrm{Cl}$ & 6.52236200 & -2.51707200 & -1.42895700 \\
\hline $\mathrm{Ti}$ & 2.52071400 & 1.57190100 & 0.33229600 \\
\hline $\mathrm{Cl}$ & 4.60718400 & 1.10413700 & 1.48775200 \\
\hline $\mathrm{Cl}$ & 0.37403400 & 1.69618500 & -0.94154800 \\
\hline C & -4.56304400 & 3.38693100 & 0.15712200 \\
\hline C & -3.59337700 & 3.52499900 & -1.05156900 \\
\hline C & -4.47925600 & 4.63546900 & 1.05421100 \\
\hline $\mathrm{H}$ & -4.23651900 & 2.51626400 & 0.75204100 \\
\hline C & -2.31206200 & 4.30759500 & -0.67691600 \\
\hline $\mathrm{H}$ & -4.08640000 & 4.03415000 & -1.90035900 \\
\hline $\mathrm{H}$ & -3.32876600 & 2.51609700 & -1.40101500 \\
\hline C & -3.07630900 & 4.73744300 & 1.70214500 \\
\hline $\mathrm{H}$ & -4.67846100 & 5.53253000 & 0.44157800 \\
\hline $\mathrm{H}$ & -5.25002500 & 4.63106400 & 1.84266700 \\
\hline C & -1.97675000 & 4.12584200 & 0.80592800 \\
\hline $\mathrm{H}$ & -2.44929700 & 5.38494400 & -0.87808800 \\
\hline $\mathrm{H}$ & -1.47449800 & 3.97291700 & -1.30813100 \\
\hline $\mathrm{H}$ & -3.08063400 & 4.22467800 & 2.67860300 \\
\hline $\mathrm{H}$ & -2.84601500 & 5.79636100 & 1.90967600 \\
\hline $\mathrm{H}$ & -1.87717600 & 3.04547600 & 1.01167100 \\
\hline $\mathrm{H}$ & -0.99756000 & 4.57009900 & 1.04823900 \\
\hline C & -7.33378400 & 4.06271200 & -1.30089300 \\
\hline $\mathrm{H}$ & -8.24939200 & 3.54143700 & -1.62306300 \\
\hline $\mathrm{H}$ & -7.61323400 & 4.89604300 & -0.63609500 \\
\hline $\mathrm{H}$ & -6.83064800 & 4.48854600 & -2.18350200 \\
\hline 0 & -6.07844300 & 1.41306200 & -1.30533900 \\
\hline 0 & -7.12266000 & 2.06113000 & 0.83077700 \\
\hline C & -6.06856200 & 1.23708300 & -2.72539100 \\
\hline $\mathrm{H}$ & -5.24959500 & 0.54922700 & -2.97661300 \\
\hline $\mathrm{H}$ & -7.03901000 & 0.81428800 & -3.02612900 \\
\hline $\mathrm{H}$ & -5.89968400 & 2.19689000 & -3.23903800 \\
\hline C & -7.19058500 & 2.39568800 & 2.21221400 \\
\hline $\mathrm{H}$ & -6.21281800 & 2.24415700 & 2.69712600 \\
\hline $\mathrm{H}$ & -7.52397800 & 3.43752700 & 2.35007600 \\
\hline $\mathrm{H}$ & -7.92463500 & 1.72590600 & 2.68232200 \\
\hline Si & -6.25398900 & 2.83738500 & -0.41415300 \\
\hline C & 3.37553400 & 3.31767700 & -0.58717600 \\
\hline $\mathrm{H}$ & 4.31659500 & 3.69163000 & -0.15676400 \\
\hline $\mathrm{H}$ & 3.66073300 & 2.31520600 & -1.03392700 \\
\hline C & 2.85022200 & 4.20208200 & -1.71647400 \\
\hline C & 3.48885400 & 3.76562800 & -3.04787300 \\
\hline C & 3.10740700 & 5.68989700 & -1.44880100 \\
\hline
\end{tabular}




$\begin{array}{lrrr}\text { H } & 1.76186500 & 4.03746000 & -1.80753700 \\ \text { H } & 3.14400300 & 4.40626500 & -3.87549400 \\ \text { H } & 3.22430900 & 2.72435300 & -3.29154700 \\ \text { H } & 4.58852100 & 3.83029500 & -2.99856400 \\ \text { H } & 2.70370800 & 6.30864400 & -2.26561500 \\ \text { H } & 4.19041100 & 5.88894700 & -1.37990400 \\ \text { H } & 2.64362200 & 6.03536700 & -0.51236800 \\ \mathrm{C} & 1.61999600 & 2.81441000 & 1.97666700 \\ \mathrm{C} & 2.07342700 & 3.84986400 & 1.15369900 \\ \mathrm{H} & 1.37690400 & 4.31159000 & 0.45169000 \\ \mathrm{H} & 2.90179000 & 4.47849600 & 1.48753900 \\ \mathrm{H} & 0.57582900 & 2.51374100 & 1.83179100 \\ \mathrm{C} & 2.22246900 & 2.51008500 & 3.33085400 \\ \mathrm{H} & 1.66428000 & 3.05086100 & 4.11573600 \\ \mathrm{H} & 2.17263800 & 1.43758100 & 3.56720800 \\ \mathrm{H} & 3.27642800 & 2.81774600 & 3.38191900 \\ \mathrm{C} & 8.10171700 & 2.72286500 & 1.23911400 \\ \mathrm{H} & 7.00622600 & 2.84816800 & 1.31254400 \\ \mathrm{H} & 8.47539600 & 3.63300500 & 0.73120900 \\ \mathrm{C} & 10.04548700 & 0.65382300 & -0.88520700 \\ \mathrm{H} & 10.36432200 & 1.50358700 & -1.51934300 \\ \mathrm{H} & 10.85033000 & 0.54025300 & -0.13273200 \\ \mathrm{C} & 9.93205600 & -0.62443700 & -1.73375700 \\ \mathrm{H} & 10.88367500 & -0.89201000 & -2.22652200 \\ \mathrm{H} & 9.62135900 & -1.48773700 & -1.12202900 \\ \mathrm{H} & 9.17159700 & -0.51495000 & -2.52472300 \\ \mathrm{C} & 8.70537400 & 2.62033400 & 2.65125600 \\ \mathrm{H} & 9.80505500 & 2.52323300 & 2.62594400 \\ \mathrm{H} & 8.47809900 & 3.50436700 & 3.27372100 \\ \mathrm{H} & 8.31691400 & 1.73781600 & 3.18695300 \\ \mathrm{Cl} & 6.66453500 & 1.13120500 & -1.47287100 \\ \mathrm{Al} & 8.40657900 & 1.17006200 & 0.06921600\end{array}$

$\mathrm{MgCl}_{2}$-Ti-isobutyl-DCPDMS-Mg2-AlEt ${ }_{2} \mathrm{Cl}$

$\begin{array}{lllr}\text { Cl } & -7.47978000 & -2.40942900 & 1.47548800 \\ \text { Mg } & -6.38476500 & -4.17636400 & 0.07626800 \\ \text { Cl } & -8.62709900 & -0.59312800 & -1.21443500 \\ \text { Mg } & -6.50890700 & -0.53835700 & 0.12264200 \\ \text { Cl } & -4.26651400 & -4.12093800 & 1.41322300 \\ \text { Cl } & -1.17749000 & -2.19367700 & 1.39731200 \\ \text { Cl } & -4.39047300 & -0.48212400 & 1.45930700 \\ \text { Cl } & -2.20081000 & -4.01593000 & -1.33877500 \\ \text { Mg } & -0.08262600 & -3.96048700 & -0.00184300\end{array}$




\begin{tabular}{|c|c|c|c|}
\hline $\mathrm{Cl}$ & -5.41374600 & -2.30425100 & -1.27685300 \\
\hline Mg & -3.29580800 & -2.24923900 & 0.06053800 \\
\hline $\mathrm{Cl}$ & -2.32483100 & -0.37737600 & -1.29257800 \\
\hline Mg & -0.20547300 & -0.32180200 & 0.04479000 \\
\hline $\mathrm{Cl}$ & 2.03559200 & -3.90500000 & 1.33517300 \\
\hline $\mathrm{Cl}$ & 5.12479800 & -1.97773400 & 1.31944100 \\
\hline $\mathrm{Cl}$ & 1.84262500 & -0.27479800 & 1.57675200 \\
\hline $\mathrm{Cl}$ & 4.10154300 & -3.80004300 & -1.41678000 \\
\hline Mg & 6.21970100 & -3.74465800 & -0.07984000 \\
\hline $\mathrm{Cl}$ & 0.88825800 & -2.08865000 & -1.35477300 \\
\hline Mg & 3.00658200 & -2.03378500 & -0.01820100 \\
\hline $\mathrm{Cl}$ & 3.85665400 & -0.03293900 & -1.31678600 \\
\hline Mg & 6.09442500 & -0.10612100 & -0.03384900 \\
\hline $\mathrm{Cl}$ & 8.21386800 & -0.05055900 & 1.30342900 \\
\hline $\mathrm{Cl}$ & 7.19059600 & -1.87276000 & -1.43278800 \\
\hline $\mathrm{Ti}$ & 2.86350500 & 1.70093300 & 0.34486500 \\
\hline $\mathrm{Cl}$ & 4.93964400 & 1.44523600 & 1.58080500 \\
\hline $\mathrm{Cl}$ & 0.66700200 & 1.74368700 & -0.81909200 \\
\hline C & 2.81936200 & 3.74605500 & 0.59211500 \\
\hline C & 3.63657600 & 3.84289800 & -0.68278200 \\
\hline $\mathrm{H}$ & 1.80411200 & 4.16300700 & 0.53496800 \\
\hline $\mathrm{H}$ & 3.34743700 & 4.05730000 & 1.50505700 \\
\hline $\mathrm{H}$ & 3.89343100 & 2.77708200 & -1.03619800 \\
\hline C & 2.87157000 & 4.45416200 & -1.86434900 \\
\hline $\mathrm{H}$ & 2.67948700 & 5.52020800 & -1.66371200 \\
\hline $\mathrm{H}$ & 3.45666400 & 4.37949900 & -2.79425100 \\
\hline $\mathrm{H}$ & 1.90551600 & 3.95370300 & -2.02152900 \\
\hline C & 5.01417100 & 4.48689600 & -0.48067500 \\
\hline $\mathrm{H}$ & 5.56509300 & 3.99295200 & 0.33005700 \\
\hline $\mathrm{H}$ & 5.62479100 & 4.41494000 & -1.39246200 \\
\hline $\mathrm{H}$ & 4.88290100 & 5.55017000 & -0.22414000 \\
\hline C & -4.77234700 & 3.37702200 & 0.57131700 \\
\hline C & -3.39944900 & 2.64436300 & 0.67611700 \\
\hline C & -4.45286500 & 4.66331100 & -0.23548100 \\
\hline $\mathrm{H}$ & -5.07014000 & 3.70589100 & 1.58510400 \\
\hline C & -2.31687200 & 3.71163700 & 0.34692800 \\
\hline $\mathrm{H}$ & -3.33953400 & 1.80067500 & -0.02248900 \\
\hline $\mathrm{H}$ & -3.27199200 & 2.20727600 & 1.67614400 \\
\hline C & -3.06191500 & 5.05530200 & 0.27660400 \\
\hline $\mathrm{H}$ & -5.21025100 & 5.45263700 & -0.10816500 \\
\hline $\mathrm{H}$ & -4.39450000 & 4.44109600 & -1.31749700 \\
\hline $\mathrm{H}$ & -1.49603300 & 3.72285600 & 1.08155700 \\
\hline $\mathrm{H}$ & -1.85526500 & 3.48684600 & -0.62775200 \\
\hline $\mathrm{H}$ & -3.15994500 & 5.49472900 & 1.28516100 \\
\hline
\end{tabular}




\begin{tabular}{|c|c|c|c|}
\hline $\mathrm{H}$ & -2.55760200 & 5.80155100 & -0.35816500 \\
\hline C & -7.50037600 & 3.55308300 & -0.85438600 \\
\hline C & -8.75806900 & 2.90789300 & -1.48252800 \\
\hline C & -8.06365200 & 4.48902200 & 0.27310200 \\
\hline $\mathrm{H}$ & -6.94668000 & 4.14833300 & -1.60070800 \\
\hline C & -9.70036800 & 2.72194100 & -0.28936900 \\
\hline $\mathrm{H}$ & -8.56958500 & 1.95707200 & -1.99984900 \\
\hline $\mathrm{H}$ & -9.19892500 & 3.61437200 & -2.20952800 \\
\hline C & -9.52161900 & 4.01115700 & 0.53426000 \\
\hline $\mathrm{H}$ & -8.04568100 & 5.53975400 & -0.05574400 \\
\hline $\mathrm{H}$ & -7.45735300 & 4.46363600 & 1.19512700 \\
\hline $\mathrm{H}$ & -10.74368400 & 2.54720000 & -0.59366600 \\
\hline $\mathrm{H}$ & -9.38138800 & 1.83135600 & 0.27407100 \\
\hline $\mathrm{H}$ & -10.22878200 & 4.78218500 & 0.18732600 \\
\hline $\mathrm{H}$ & -9.72530400 & 3.85922600 & 1.60648800 \\
\hline 0 & -5.78210000 & 1.09672500 & -1.08751900 \\
\hline 0 & -6.90513300 & 1.35213800 & 1.06719800 \\
\hline C & -7.04680900 & 1.55201300 & 2.46868700 \\
\hline $\mathrm{H}$ & -6.06106200 & 1.64759700 & 2.95146700 \\
\hline $\mathrm{H}$ & -7.65911400 & 2.44422600 & 2.67704900 \\
\hline $\mathrm{H}$ & -7.55538200 & 0.66938400 & 2.88165000 \\
\hline C & -5.68763600 & 1.02629700 & -2.51267500 \\
\hline $\mathrm{H}$ & -4.72821000 & 0.55524100 & -2.76964800 \\
\hline $\mathrm{H}$ & -6.52890000 & 0.42010200 & -2.88111900 \\
\hline $\mathrm{H}$ & -5.73257900 & 2.03105000 & -2.96270700 \\
\hline Si & -6.23080800 & 2.39878200 & -0.10220700 \\
\hline C & 8.42143600 & 3.37561600 & 1.35433700 \\
\hline $\mathrm{H}$ & 7.36604600 & 3.26910100 & 1.66322500 \\
\hline $\mathrm{H}$ & 8.47145700 & 4.32390900 & 0.78573300 \\
\hline C & 10.47345000 & 1.48317200 & -0.81675500 \\
\hline $\mathrm{H}$ & 10.73436700 & 2.36732100 & -1.43013500 \\
\hline $\mathrm{H}$ & 11.28521200 & 1.40605400 & -0.06723900 \\
\hline C & 10.44480100 & 0.22098200 & -1.69567400 \\
\hline $\mathrm{H}$ & 11.41074900 & 0.03096800 & -2.19645300 \\
\hline $\mathrm{H}$ & 10.19600700 & -0.67592900 & -1.10427400 \\
\hline $\mathrm{H}$ & 9.67581300 & 0.29689300 & -2.48223200 \\
\hline C & 9.31919900 & 3.45710100 & 2.60225200 \\
\hline $\mathrm{H}$ & 10.38297800 & 3.59137700 & 2.33885300 \\
\hline $\mathrm{H}$ & 9.04619000 & 4.29678600 & 3.26603000 \\
\hline $\mathrm{H}$ & 9.25115400 & 2.53572300 & 3.20605100 \\
\hline $\mathrm{Cl}$ & 7.06502400 & 1.72748400 & -1.38428300 \\
\hline $\mathrm{Al}$ & 8.80606400 & 1.87156000 & 0.14832800 \\
\hline
\end{tabular}

$\mathrm{MgCl}_{2}$-Ti-isobutyl-DCPDMS-Mg2-AlEt ${ }_{2} \mathrm{Cl}$-Propylene-1, 2-re-coordination 


\begin{tabular}{|c|c|c|c|}
\hline $\mathrm{Cl}$ & 7.54019900 & -2.48378300 & -1.54054100 \\
\hline Mg & 6.48421600 & -4.26443700 & -0.12876700 \\
\hline $\mathrm{Cl}$ & 8.69383700 & -0.65174300 & 1.13597200 \\
\hline Mg & 6.56020100 & -0.62513400 & -0.17719100 \\
\hline $\mathrm{Cl}$ & 4.35051300 & -4.23717400 & -1.44180800 \\
\hline $\mathrm{Cl}$ & 1.23688500 & -2.35051500 & -1.39146700 \\
\hline $\mathrm{Cl}$ & 4.42630600 & -0.59706600 & -1.48993900 \\
\hline $\mathrm{Cl}$ & 2.31470500 & -4.15830500 & 1.33324600 \\
\hline Mg & 0.18105800 & -4.13103800 & 0.02024000 \\
\hline $\mathrm{Cl}$ & 5.50406200 & -2.40475000 & 1.23485800 \\
\hline Mg & 3.37065600 & -2.37792200 & -0.07859700 \\
\hline $\mathrm{Cl}$ & 2.39058300 & -0.51841500 & 1.28496900 \\
\hline Mg & 0.25605500 & -0.49117200 & -0.02835000 \\
\hline $\mathrm{Cl}$ & -1.95262100 & -4.10370800 & -1.29287600 \\
\hline $\mathrm{Cl}$ & -5.06640000 & -2.21702800 & -1.24267500 \\
\hline $\mathrm{Cl}$ & -1.81431100 & -0.50934000 & -1.47490500 \\
\hline $\mathrm{Cl}$ & -3.98865400 & -4.02488300 & 1.48214300 \\
\hline Mg & -6.12225700 & -3.99765900 & 0.16912100 \\
\hline $\mathrm{Cl}$ & -0.79897400 & -2.27160000 & 1.38369500 \\
\hline Mg & -2.93260200 & -2.24470700 & 0.07076800 \\
\hline $\mathrm{Cl}$ & -3.80811400 & -0.27602000 & 1.36663100 \\
\hline Mg & -6.04548900 & -0.35780300 & 0.12102100 \\
\hline $\mathrm{Cl}$ & -8.18008600 & -0.33048500 & -1.19222300 \\
\hline $\mathrm{Cl}$ & -7.10228300 & -2.13820100 & 1.53255400 \\
\hline $\mathrm{Ti}$ & -2.93418600 & 1.63540000 & -0.14742000 \\
\hline $\mathrm{Cl}$ & -4.89277900 & 1.22379900 & -1.48412200 \\
\hline $\mathrm{Cl}$ & -0.60283800 & 1.61558500 & 0.79085100 \\
\hline C & 4.75915500 & 3.28585700 & -0.58502100 \\
\hline C & 3.41770800 & 2.53825000 & -0.82167500 \\
\hline C & 4.34397000 & 4.48993600 & 0.29756900 \\
\hline $\mathrm{H}$ & 5.10555700 & 3.70433600 & -1.55053500 \\
\hline C & 2.29726100 & 3.60965800 & -0.66927300 \\
\hline $\mathrm{H}$ & 3.28887800 & 1.73679900 & -0.08126600 \\
\hline $\mathrm{H}$ & 3.40576700 & 2.04043000 & -1.80026300 \\
\hline C & 3.01465900 & 4.93283600 & -0.32715700 \\
\hline $\mathrm{H}$ & 5.09840900 & 5.29172100 & 0.32573300 \\
\hline $\mathrm{H}$ & 4.17490000 & 4.16274400 & 1.34059900 \\
\hline $\mathrm{H}$ & 1.68469500 & 3.70439100 & -1.58107800 \\
\hline $\mathrm{H}$ & 1.60931500 & 3.32343000 & 0.14054000 \\
\hline $\mathrm{H}$ & 3.22733700 & 5.50384500 & -1.24768300 \\
\hline $\mathrm{H}$ & 2.42381900 & 5.58869200 & 0.33214000 \\
\hline C & 7.44927500 & 3.46392400 & 0.89566900 \\
\hline C & 8.66690800 & 2.80952500 & 1.60434000 \\
\hline C & 8.06412000 & 4.39936400 & -0.21187800 \\
\hline
\end{tabular}




\begin{tabular}{|c|c|c|c|}
\hline $\mathrm{H}$ & 6.86614600 & 4.06487800 & 1.61356200 \\
\hline C & 9.76481800 & 2.81837500 & 0.53739400 \\
\hline $\mathrm{H}$ & 8.47996700 & 1.79436600 & 1.97864900 \\
\hline $\mathrm{H}$ & 8.97042500 & 3.44031500 & 2.45870400 \\
\hline C & 9.59494100 & 4.19004300 & -0.12874400 \\
\hline $\mathrm{H}$ & 7.77897600 & 5.45208300 & -0.06117500 \\
\hline $\mathrm{H}$ & 7.70534100 & 4.13859300 & -1.22303700 \\
\hline $\mathrm{H}$ & 10.77036800 & 2.65699300 & 0.95567300 \\
\hline $\mathrm{H}$ & 9.57676400 & 1.99867300 & -0.17565300 \\
\hline $\mathrm{H}$ & 10.03967500 & 4.96866300 & 0.51471400 \\
\hline $\mathrm{H}$ & 10.08514300 & 4.26673500 & -1.11254200 \\
\hline 0 & 5.78692500 & 0.98083700 & 1.05352300 \\
\hline 0 & 6.91844000 & 1.29888300 & -1.09392200 \\
\hline C & 6.97989500 & 1.48267200 & -2.50490400 \\
\hline $\mathrm{H}$ & 5.97020300 & 1.46309000 & -2.94506500 \\
\hline $\mathrm{H}$ & 7.48528500 & 2.42822400 & -2.75848800 \\
\hline $\mathrm{H}$ & 7.56123000 & 0.64875100 & -2.92347100 \\
\hline C & 5.68829800 & 0.89255700 & 2.47762700 \\
\hline $\mathrm{H}$ & 4.72970500 & 0.41636600 & 2.72669300 \\
\hline $\mathrm{H}$ & 6.52992100 & 0.28437000 & 2.84182900 \\
\hline $\mathrm{H}$ & 5.73090200 & 1.89170100 & 2.94012600 \\
\hline Si & 6.21560700 & 2.30747600 & 0.09293000 \\
\hline C & -3.44973000 & 3.11397200 & 1.18917300 \\
\hline $\mathrm{H}$ & -4.32128000 & 3.45005200 & 0.58403900 \\
\hline $\mathrm{H}$ & -3.87568800 & 2.56032000 & 2.04617900 \\
\hline C & -2.55364600 & 4.27652700 & 1.64791600 \\
\hline C & -1.97497000 & 4.00205400 & 3.04089300 \\
\hline C & -3.30739200 & 5.61465000 & 1.61418900 \\
\hline $\mathrm{H}$ & -1.69273400 & 4.36226900 & 0.95710800 \\
\hline $\mathrm{H}$ & -1.27441800 & 4.79730400 & 3.34357400 \\
\hline $\mathrm{H}$ & -1.43376300 & 3.04502800 & 3.06402700 \\
\hline $\mathrm{H}$ & -2.78247000 & 3.95734100 & 3.79159200 \\
\hline $\mathrm{H}$ & -2.65786800 & 6.44826800 & 1.92961400 \\
\hline $\mathrm{H}$ & -4.17832200 & 5.58721700 & 2.29024700 \\
\hline $\mathrm{H}$ & -3.68284100 & 5.84365000 & 0.60240500 \\
\hline C & -1.84710900 & 2.84250200 & -2.18403600 \\
\hline $\mathrm{H}$ & -2.12038900 & 2.13344400 & -2.97255300 \\
\hline $\mathrm{H}$ & -0.84509800 & 2.75919800 & -1.75594500 \\
\hline C & -2.66503500 & 3.86493800 & -1.84508800 \\
\hline $\mathrm{H}$ & -2.31418500 & 4.56410500 & -1.07697300 \\
\hline C & -3.97411600 & 4.20490500 & -2.48373400 \\
\hline $\mathrm{H}$ & -4.77381900 & 4.30353500 & -1.73267700 \\
\hline $\mathrm{H}$ & -3.88121600 & 5.18626700 & -2.98107400 \\
\hline $\mathrm{H}$ & -4.28735600 & 3.45580100 & -3.22263300 \\
\hline & & & \\
\hline
\end{tabular}




$\begin{array}{lrrr}\text { C } & -8.03732600 & 3.15104700 & -1.40939600 \\ \text { H } & -7.03091800 & 3.44050900 & -1.05402000 \\ \text { H } & -8.67550800 & 4.04073200 & -1.24677200 \\ \text { C } & -10.37636700 & 1.51430000 & 0.70951900 \\ \text { H } & -10.60283300 & 2.45208600 & 1.25269900 \\ \text { H } & -11.15972500 & 1.43818100 & -0.06978200 \\ \text { C } & -10.46598700 & 0.31343500 & 1.66771100 \\ \text { H } & -11.46365900 & 0.21443100 & 2.13095100 \\ \text { H } & -10.24734700 & -0.63412400 & 1.14728900 \\ \text { H } & -9.73036100 & 0.39708900 & 2.48476600 \\ \text { C } & -7.97373300 & 2.81825100 & -2.90974900 \\ \text { H } & -8.96715100 & 2.56368400 & -3.31743500 \\ \text { H } & -7.58687100 & 3.66136300 & -3.51050200 \\ \text { H } & -7.31940600 & 1.95263100 & -3.10015800 \\ \text { Cl } & -6.99480200 & 1.51103300 & 1.43290200 \\ \text { Al } & -8.64287600 & 1.71641500 & -0.19367500\end{array}$

$\mathrm{MgCl}_{2}$-Ti-i sobutyl-DCPDMS-Mg2-AlEt ${ }_{2} \mathrm{Cl}_{\text {-Propylene-1, 2-re-TS }}$

$\begin{array}{lrrr}\text { Cl } & 7.57231900 & -2.43299500 & -1.51661300 \\ \text { Mg } & 6.51833900 & -4.23318600 & -0.12831300 \\ \text { Cl } & 8.70763400 & -0.62510500 & 1.18404600 \\ \text { Mg } & 6.57909100 & -0.59328400 & -0.13724300 \\ \text { Cl } & 4.38972800 & -4.20071400 & -1.44947500 \\ \text { Cl } & 1.26795200 & -2.32777500 & -1.39124200 \\ \text { Cl } & 4.45028300 & -0.56001200 & -1.45810200 \\ \text { Cl } & 2.34269100 & -4.16032100 & 1.31813700 \\ \text { Mg } & 0.21413400 & -4.12784900 & -0.00298300 \\ \text { Cl } & 5.52495100 & -2.39244200 & 1.25134400 \\ \text { Mg } & 3.39665500 & -2.36037300 & -0.07025800 \\ \text { Cl } & 2.40334000 & -0.51981500 & 1.30935000 \\ \text { Mg } & 0.27397200 & -0.48764100 & -0.01194200 \\ \text { Cl } & -1.91446800 & -4.09532900 & -1.32420200 \\ \text { Cl } & -5.03635000 & -2.22240300 & -1.26605200 \\ \text { Cl } & -1.77088200 & -0.52839800 & -1.51249700 \\ \text { Cl } & -3.96169700 & -4.05496500 & 1.44335500 \\ \text { Mg } & -6.09020400 & -4.02251500 & 0.12224100 \\ \text { Cl } & -0.77912000 & -2.28736600 & 1.37649400 \\ \text { Mg } & -2.90754200 & -2.25500300 & 0.05532200 \\ \text { Cl } & -3.76727600 & -0.29995900 & 1.33257700 \\ \text { Mg } & -6.02905200 & -0.38195600 & 0.11350600 \\ \text { Cl } & -8.15818000 & -0.34956600 & -1.20769400 \\ \text { Cl } & -7.08341700 & -2.18206200 & 1.50165500 \\ \text { Ti } & -2.82464200 & 1.56805400 & -0.34384500 \\ \text { Cl } & -4.94653800 & 1.22731700 & -1.50019900\end{array}$




\begin{tabular}{|c|c|c|c|}
\hline $\mathrm{Cl}$ & -0.66134200 & 1.58086800 & 0.88329800 \\
\hline C & 4.76074400 & 3.31653100 & -0.47691900 \\
\hline C & 3.42845200 & 2.55307300 & -0.71701000 \\
\hline C & 4.33784500 & 4.48784200 & 0.44543000 \\
\hline $\mathrm{H}$ & 5.08698100 & 3.76670500 & -1.43526500 \\
\hline C & 2.29103600 & 3.59737500 & -0.51134600 \\
\hline $\mathrm{H}$ & 3.32526800 & 1.72629500 & -0.00157600 \\
\hline $\mathrm{H}$ & 3.40863000 & 2.08815600 & -1.71156500 \\
\hline C & 2.99085300 & 4.92348600 & -0.14602700 \\
\hline $\mathrm{H}$ & 5.07724800 & 5.30283500 & 0.48635900 \\
\hline $\mathrm{H}$ & 4.19060800 & 4.12786800 & 1.48090000 \\
\hline $\mathrm{H}$ & 1.65645600 & 3.70505700 & -1.40650400 \\
\hline $\mathrm{H}$ & 1.62594100 & 3.27531000 & 0.30453300 \\
\hline $\mathrm{H}$ & 3.17859500 & 5.52191000 & -1.05453600 \\
\hline $\mathrm{H}$ & 2.40020000 & 5.55184600 & 0.53978600 \\
\hline C & 7.46497000 & 3.49084400 & 0.98139000 \\
\hline C & 8.75393700 & 2.87541400 & 1.56841100 \\
\hline C & 8.02410800 & 4.54302800 & -0.01727800 \\
\hline $\mathrm{H}$ & 6.86842100 & 3.98969500 & 1.76728200 \\
\hline C & 9.70030700 & 2.75253300 & 0.36061600 \\
\hline $\mathrm{H}$ & 8.61111400 & 1.90369600 & 2.06124200 \\
\hline $\mathrm{H}$ & 9.16581500 & 3.58091900 & 2.31267200 \\
\hline C & 9.30389200 & 3.89796500 & -0.61109200 \\
\hline H & 8.28896500 & 5.45378400 & 0.54550500 \\
\hline $\mathrm{H}$ & 7.30494400 & 4.86024500 & -0.79123400 \\
\hline $\mathrm{H}$ & 10.75736900 & 2.80091200 & 0.66325700 \\
\hline $\mathrm{H}$ & 9.54813900 & 1.76666300 & -0.10255300 \\
\hline $\mathrm{H}$ & 10.10092500 & 4.65001800 & -0.72059400 \\
\hline $\mathrm{H}$ & 9.11605400 & 3.50305100 & -1.62224700 \\
\hline 0 & 5.81966700 & 1.00221400 & 1.10709000 \\
\hline 0 & 6.92016700 & 1.34260100 & -1.05268100 \\
\hline C & 6.98492100 & 1.57631500 & -2.45652100 \\
\hline $\mathrm{H}$ & 6.02914600 & 1.30365200 & -2.93066300 \\
\hline $\mathrm{H}$ & 7. 22416000 & 2.62939600 & -2.67477200 \\
\hline $\mathrm{H}$ & 7.78294000 & 0.94124800 & -2.86706000 \\
\hline C & 5.72674800 & 0.89648000 & 2.53050200 \\
\hline $\mathrm{H}$ & 4.78135000 & 0.39205700 & 2.77477500 \\
\hline $\mathrm{H}$ & 6.58483600 & 0.30691300 & 2.88681400 \\
\hline $\mathrm{H}$ & 5.74134900 & 1.89154800 & 3.00320200 \\
\hline Si & 6.23786900 & 2.33745100 & 0.15454400 \\
\hline C & -3.52989500 & 3.40528600 & 0.53614500 \\
\hline $\mathrm{H}$ & -4.49219900 & 3.82951400 & 0.21845300 \\
\hline $\mathrm{H}$ & -3.83831800 & 2.44734600 & 1.05740700 \\
\hline C & -2.79563000 & 4.29224600 & 1.5448260 \\
\hline
\end{tabular}




$\begin{array}{llll}\text { C } & -3.18554100 & 3.88596600 & 2.97705900 \\ \text { C } & -3.06710100 & 5.78265800 & 1.30603000 \\ \text { H } & -1.71096300 & 4.11266300 & 1.44205400 \\ \text { H } & -2.67705800 & 4.52282800 & 3.71885800 \\ \text { H } & -2.90474100 & 2.84017200 & 3.17918600 \\ \text { H } & -4.27345300 & 3.98188900 & 3.13128900 \\ \text { H } & -2.52220500 & 6.40049300 & 2.03714400 \\ \text { H } & -4.14256000 & 6.00495500 & 1.41287200 \\ \text { H } & -2.75660000 & 6.11172400 & 0.30210100 \\ \text { C } & -1.96854600 & 2.66375900 & -2.05172100 \\ \text { H } & -2.44602100 & 2.21202600 & -2.92760300 \\ \text { H } & -0.90171600 & 2.46478500 & -1.91454800 \\ \text { C } & -2.52341500 & 3.81851300 & -1.48013400 \\ \text { H } & -1.84683300 & 4.44321200 & -0.89133500 \\ \text { C } & -3.68907400 & 4.51906800 & -2.12904900 \\ \text { H } & -4.15629100 & 5.26690400 & -1.47551400 \\ \text { H } & -3.31079100 & 5.04100700 & -3.02497800 \\ \text { H } & -4.45469500 & 3.79934400 & -2.45031400 \\ \text { C } & -8.10415800 & 3.13973200 & -1.34230600 \\ \text { H } & -7.09122000 & 3.44123400 & -1.01763300 \\ \text { H } & -8.75489400 & 4.01130600 & -1.13587200 \\ \text { C } & -10.36034800 & 1.39909000 & 0.77573400 \\ \text { H } & -10.59493700 & 2.31764900 & 1.34775400 \\ \text { H } & -11.15544300 & 1.32913200 & 0.00776100 \\ \text { C } & -10.41182000 & 0.17190900 & 1.70254100 \\ \text { H } & -11.39898700 & 0.04242800 & 2.18075700 \\ \text { H } & -10.18572500 & -0.75722900 & 1.15301200 \\ \text { H } & -9.66280600 & 0.24707800 & 2.50818100 \\ \text { C } & -8.08728300 & 2.84268300 & -2.85179400 \\ \text { H } & -9.08682000 & 2.56584300 & -3.22936900 \\ \text { H } & -7.74935600 & 3.71087900 & -3.44622000 \\ \text { H } & -7.41314300 & 2.00306500 & -3.08480900 \\ \text { Al } & -6.97744700 & 1.46300100 & 1.46222300 \\ & -8.64662600 & 1.66134900 & -0.15141900\end{array}$

$\mathrm{MgCl}_{2}$-Ti-isobutyl-DCPDMS-Mg2-AlEt ${ }_{2} \mathrm{Cl}$-Propylene-1,2-si-coordination

$\begin{array}{lllr}\text { Cl } & 7.62171700 & -2.43922600 & -1.46801600 \\ \text { Mg } & 6.54321900 & -4.24525900 & -0.10640200 \\ \text { Cl } & 8.73634500 & -0.65958300 & 1.25986100 \\ \text { Mg } & 6.62273000 & -0.60577900 & -0.08447200 \\ \text { Cl } & 4.42955500 & -4.19080600 & -1.45061800 \\ \text { Cl } & 1.31697200 & -2.30215100 & -1.41126000 \\ \text { Cl } & 4.50886600 & -0.55051900 & -1.42840000 \\ \text { Cl } & 2.35226200 & -4.16252000 & 1.29450700\end{array}$




\begin{tabular}{|c|c|c|c|}
\hline Mg & 0.23861700 & -4.10807000 & -0.04969200 \\
\hline $\mathrm{Cl}$ & 5.54409200 & -2.41077900 & 1.27744600 \\
\hline Mg & 3.43070800 & -2.35674900 & -0.06718200 \\
\hline $\mathrm{Cl}$ & 2.43166000 & -0.52245600 & 1.31657600 \\
\hline Mg & 0.31733200 & -0.46806700 & -0.02788600 \\
\hline $\mathrm{Cl}$ & -1.87500200 & -4.05355000 & -1.39396600 \\
\hline $\mathrm{Cl}$ & -4.98772600 & -2.16490800 & -1.35470400 \\
\hline $\mathrm{Cl}$ & -1.76162000 & -0.48844700 & -1.49687306 \\
\hline $\mathrm{Cl}$ & -3.95251600 & -4.02530100 & $1.3511140 e$ \\
\hline Mg & -6.06607800 & -3.97088700 & 0.00692200 \\
\hline $\mathrm{Cl}$ & -0.76037800 & -2.27382900 & 1.33400000 \\
\hline Mg & -2.87393100 & -2.21962700 & -0.0102140 \\
\hline $\mathrm{Cl}$ & -3.75842200 & -0.22565900 & $1.3018440 e$ \\
\hline Mg & -5.98607400 & -0.33081500 & 0.02914700 \\
\hline $\mathrm{Cl}$ & -8.10036300 & -0.27637600 & -1.31525200 \\
\hline $\mathrm{Cl}$ & -7.06506400 & -2.13666300 & 1.39054500 \\
\hline $\mathrm{Ti}$ & -2.81163100 & 1.69338100 & -0.16978000 \\
\hline $\mathrm{Cl}$ & -4.86993200 & 1.45199000 & $-1.4026080 e$ \\
\hline $\mathrm{Cl}$ & -0.40770800 & 1.71397900 & 0.62505900 \\
\hline C & 4.89654900 & 3.31736800 & -0.55814400 \\
\hline C & 3.52877000 & 2.60441900 & -0.75922300 \\
\hline C & 4.52760900 & 4.58972000 & 0.24547100 \\
\hline $\mathrm{H}$ & 5.25850000 & 3.66304100 & -1.54633400 \\
\hline C & 2.44689600 & 3.71853100 & -0.63821200 \\
\hline $\mathrm{H}$ & 3.37540300 & 1.82618800 & 0.00188100 \\
\hline $\mathrm{H}$ & 3.49564900 & 2.07999800 & -1.72314000 \\
\hline C & 3.21359200 & 5.03625400 & -0.40684300 \\
\hline $\mathrm{H}$ & 5.31149100 & 5.36271800 & 0.22151800 \\
\hline $\mathrm{H}$ & 4.34850900 & 4.33808400 & 1.30750300 \\
\hline $\mathrm{H}$ & 1.80269300 & 3.77076900 & -1.53082800 \\
\hline $\mathrm{H}$ & 1.78322000 & 3.50601800 & 0.21327700 \\
\hline $\mathrm{H}$ & 3.44213400 & 5.52318200 & -1.37108100 \\
\hline $\mathrm{H}$ & 2.65210300 & 5.76456000 & 0.20010100 \\
\hline C & 7.55209500 & 3.46922300 & 0.99199100 \\
\hline C & 8.74725200 & 2.80200100 & 1.72505600 \\
\hline C & 8.20071900 & 4.38945400 & -0.10895900 \\
\hline $\mathrm{H}$ & 6.96235900 & 4.08157600 & 1.69489700 \\
\hline C & 9.85486900 & 2.76885300 & 0.66858900 \\
\hline $\mathrm{H}$ & 8.53479200 & 1.79771100 & 2.11502500 \\
\hline $\mathrm{H}$ & 9.05551400 & 3.44129700 & 2.57148000 \\
\hline C & 9.72500600 & 4.13429200 & -0.01928100 \\
\hline $\mathrm{H}$ & 7.94842800 & 5.44970000 & 0.04709300 \\
\hline $\mathrm{H}$ & 7.83669800 & 4.14665500 & -1.12275400 \\
\hline $\mathrm{H}$ & 10.85229200 & 2.58938100 & 1.09867900 \\
\hline
\end{tabular}




\begin{tabular}{|c|c|c|c|}
\hline $\mathrm{H}$ & 9.65317000 & 1.94294800 & -0.03315100 \\
\hline $\mathrm{H}$ & 10.19201000 & 4.90990500 & 0.61172400 \\
\hline $\mathrm{H}$ & 10.21866500 & 4.18032200 & -1.00322700 \\
\hline 0 & 5.85087200 & 1.01026200 & 1.12703500 \\
\hline 0 & 7.03138200 & 1.30165900 & -0.99817300 \\
\hline C & 7.12698100 & 1.47512100 & -2.40835600 \\
\hline $\mathrm{H}$ & 6.12650300 & 1.47623100 & -2.86938400 \\
\hline $\mathrm{H}$ & 7.65983000 & 2.40704000 & -2.65620600 \\
\hline $\mathrm{H}$ & 7.69661400 & 0.62470200 & -2.80929900 \\
\hline C & 5.72233500 & 0.92142500 & 2.54870600 \\
\hline $\mathrm{H}$ & 4.75527900 & 0.45156200 & 2.77662700 \\
\hline $\mathrm{H}$ & 6.55189400 & 0.30669400 & 2.92944600 \\
\hline $\mathrm{H}$ & 5.76242100 & 1.91965200 & 3.01342500 \\
\hline Si & 6.31944800 & 2.32577800 & 0.16955500 \\
\hline C & -3.06600300 & 3.17235600 & 1.25428200 \\
\hline $\mathrm{H}$ & -2.32089600 & 3.98402900 & 1.19998500 \\
\hline $\mathrm{H}$ & -2.76571700 & 2.53912200 & 2.11639300 \\
\hline C & -4.50026900 & 3.68752500 & 1.44770200 \\
\hline C & -4.67256700 & 4.27848500 & 2.85714400 \\
\hline $\mathrm{H}$ & -5.71333500 & 4.60382900 & 3.02164300 \\
\hline $\mathrm{H}$ & -4.01313300 & 5.15183900 & 3.00067600 \\
\hline $\mathrm{H}$ & -4.42472900 & 3.53687300 & 3.63302200 \\
\hline C & -1.78340400 & 2.67439100 & -2.33912300 \\
\hline $\mathrm{H}$ & -2.40853000 & 1.98449300 & -2.91327000 \\
\hline $\mathrm{H}$ & -0.72663600 & 2.41841200 & -2.22376100 \\
\hline C & -2.26838800 & 3.85887300 & -1.90716200 \\
\hline $\mathrm{H}$ & -3.32274000 & 4.08732700 & -2.09986300 \\
\hline C & -1.44370800 & 4.93722800 & -1.27619800 \\
\hline $\mathrm{H}$ & -1.98722900 & 5.44186900 & -0.46552800 \\
\hline $\mathrm{H}$ & -0.49061100 & 4.54901100 & -0.88920600 \\
\hline $\mathrm{H}$ & -1.22223500 & 5.70574200 & -2.03823300 \\
\hline $\mathrm{H}$ & -5.18765800 & 2.83180400 & 1.37838900 \\
\hline C & -4.92695900 & 4.69730800 & 0.37817000 \\
\hline $\mathrm{H}$ & -4.91605300 & 4.24622000 & -0.62457000 \\
\hline $\mathrm{H}$ & -5.95472500 & 5.04840800 & 0.56031600 \\
\hline $\mathrm{H}$ & -4.26622800 & 5.58179800 & 0.37368200 \\
\hline C & -8.42931300 & 3.13668900 & -1.06726400 \\
\hline $\mathrm{H}$ & -7.32809200 & 3.21764300 & -1.10754000 \\
\hline $\mathrm{H}$ & -8.76899200 & 4.02159300 & -0.49502200 \\
\hline C & -10.53899800 & 0.97879500 & 0.78830200 \\
\hline $\mathrm{H}$ & -10.83307700 & 1.76354600 & 1.51207600 \\
\hline $\mathrm{H}$ & -11.31984000 & 1.00738500 & 0.00344300 \\
\hline C & -10.53300200 & -0.39682500 & 1.47710100 \\
\hline $\mathrm{H}$ & -11.51232900 & -0.65405000 & 1.91845400 \\
\hline
\end{tabular}




$\begin{array}{lrrr}\text { H } & -10.26839100 & -1.19899900 & 0.76812800 \\ \text { H } & -9.78503500 & -0.43917900 & 2.28602800 \\ \text { C } & -9.00645400 & 3.17527300 & -2.49317000 \\ \text { H } & -10.10962300 & 3.12778800 & -2.49384900 \\ \text { H } & -8.72663500 & 4.09403900 & -3.03924900 \\ \text { H } & -8.64837500 & 2.32059400 & -3.09190200 \\ \text { Cl } & -7.17021600 & 1.28137900 & 1.54872900 \\ \text { Al } & -8.83404300 & 1.51254100 & -0.03145100\end{array}$

\begin{tabular}{|c|c|c|c|}
\hline \multicolumn{4}{|c|}{$\mathrm{MgCl}_{2}$-Ti-isobutyl-DCPDMS-Mg2-AlEt ${ }_{2} \mathrm{Cl}$-Propylene-1,2-si-TS } \\
\hline $\mathrm{Cl}$ & -7.64012200 & -2.37769000 & 1.49274800 \\
\hline Mg & -6.58294900 & -4.20387300 & 0.14132400 \\
\hline $\mathrm{Cl}$ & -8.74768100 & -0.60859900 & -1.24485100 \\
\hline Mg & -6.62906200 & -0.56408600 & 0.09192500 \\
\hline $\mathrm{Cl}$ & -4.46427600 & -4.15871400 & 1.47797900 \\
\hline $\mathrm{Cl}$ & -1.33460600 & -2.29919100 & 1.41378300 \\
\hline $\mathrm{Cl}$ & -4.51018500 & -0.51812300 & 1.42828400 \\
\hline $\mathrm{Cl}$ & -2.39623700 & -4.17042100 & -1.27424400 \\
\hline Mg & -0.27757300 & -4.12526300 & 0.06240100 \\
\hline $\mathrm{Cl}$ & -5.57174500 & -2.38924100 & -1.25981000 \\
\hline Mg & -3.45336000 & -2.34449100 & 0.07726500 \\
\hline $\mathrm{Cl}$ & -2.44225100 & -0.53003900 & -1.32376100 \\
\hline Mg & -0.32290800 & -0.48503500 & 0.01299300 \\
\hline $\mathrm{Cl}$ & 1.84106000 & -4.08004500 & 1.39910400 \\
\hline $\mathrm{Cl}$ & 4.97086600 & -2.22056700 & 1.33494100 \\
\hline $\mathrm{Cl}$ & 1.73680200 & -0.51314300 & 1.50608400 \\
\hline $\mathrm{Cl}$ & 3.90930800 & -4.09177900 & -1.35307300 \\
\hline Mg & 6.02785300 & -4.04665700 & -0.01646000 \\
\hline $\mathrm{Cl}$ & 0.73347400 & -2.31088100 & -1.33858700 \\
\hline Mg & 2.85192300 & -2.26590300 & -0.00184200 \\
\hline $\mathrm{Cl}$ & 3.72283200 & -0.32374900 & -1.32115400 \\
\hline Mg & 5.98143000 & -0.40619700 & -0.06603300 \\
\hline $\mathrm{Cl}$ & 8.10056300 & -0.36114100 & 1.27065400 \\
\hline $\mathrm{Cl}$ & 7.03891500 & -2.23230200 & -1.41733400 \\
\hline $\mathrm{Ti}$ & 2.75219600 & 1.58043200 & 0.27396900 \\
\hline $\mathrm{Cl}$ & 4.93347900 & 1.34374200 & 1.38498500 \\
\hline $\mathrm{Cl}$ & 0.55607000 & 1.60598000 & -0.86262100 \\
\hline C & -4.85852000 & 3.35649600 & 0.42195500 \\
\hline C & -3.52353400 & 2.59831500 & 0.66372500 \\
\hline C & -4.44007100 & 4.53579100 & -0.49182100 \\
\hline $\mathrm{H}$ & -5.19077700 & 3.79914400 & 1.38162400 \\
\hline C & -2.39216600 & 3.64819800 & 0.45957300 \\
\hline $\mathrm{H}$ & -3.41357400 & 1.76933500 & -0.04861600 \\
\hline $\mathrm{H}$ & -3.50272200 & 2.13599100 & 1.65932300 \\
\hline
\end{tabular}




\begin{tabular}{|c|c|c|c|}
\hline C & -3.09725400 & 4.97429400 & 0.10669400 \\
\hline $\mathrm{H}$ & -5.18425600 & 5.34653600 & -0.52959400 \\
\hline $\mathrm{H}$ & -4.28753900 & 4.18379600 & -1.52925600 \\
\hline $\mathrm{H}$ & -1.75408000 & 3.75044100 & 1.35176800 \\
\hline $\mathrm{H}$ & -1.73220200 & 3.33343400 & -0.36335200 \\
\hline $\mathrm{H}$ & -3.29016400 & 5.56210600 & 1.02106000 \\
\hline $\mathrm{H}$ & -2.50793000 & 5.61267400 & -0.57090600 \\
\hline C & -7.55270000 & 3.50408200 & -1.05712300 \\
\hline C & -8.83528300 & 2.87974600 & -1.64834000 \\
\hline C & -8.12303500 & 4.55791600 & -0.06611800 \\
\hline $\mathrm{H}$ & -6.95391700 & 4.00287500 & -1.84149500 \\
\hline C & -9.78479700 & 2.75416600 & -0.44353300 \\
\hline $\mathrm{H}$ & -8.68446100 & 1.90781200 & -2.13829100 \\
\hline $\mathrm{H}$ & -9.24842100 & 3.58149300 & -2.39549100 \\
\hline C & -9.40367900 & 3.90948800 & 0.52241100 \\
\hline $\mathrm{H}$ & -8.38830600 & 5.46500500 & -0.63454006 \\
\hline $\mathrm{H}$ & -7.41043400 & 4.88189000 & 0.71110100 \\
\hline $\mathrm{H}$ & -10.84132300 & 2.78912000 & -0.74985900 \\
\hline $\mathrm{H}$ & -9.62304700 & 1.77289500 & 0.02617800 \\
\hline $\mathrm{H}$ & -10.20624600 & 4.65741400 & 0.61879000 \\
\hline $\mathrm{H}$ & -9.22201800 & 3.52403900 & 1.53836000 \\
\hline 0 & -5.87791400 & 1.02886100 & -1.15676100 \\
\hline 0 & -6.99803500 & 1.36936200 & 0.99341600 \\
\hline C & -7.07066900 & 1.60731700 & 2.39614500 \\
\hline $\mathrm{H}$ & -6.11076000 & 1.35552600 & 2.87350400 \\
\hline $\mathrm{H}$ & -7.33209100 & 2.65610200 & 2.60944600 \\
\hline $\mathrm{H}$ & -7.85624900 & 0.95726700 & 2.80708000 \\
\hline C & -5.77089700 & 0.91105200 & -2.57827300 \\
\hline $\mathrm{H}$ & -4.82533100 & 0.39979200 & -2.80754900 \\
\hline $\mathrm{H}$ & -6.62798800 & 0.32264200 & -2.93901100 \\
\hline $\mathrm{H}$ & -5.77476900 & 1.90231700 & -3.05915000 \\
\hline $\mathrm{Si}$ & -6.32245100 & 2.36462200 & -0.21672900 \\
\hline C & 3.29209400 & 3.46088900 & -0.66033600 \\
\hline $\mathrm{H}$ & 2.48314100 & 3.97236200 & -1.20393200 \\
\hline $\mathrm{H}$ & 3.47991500 & 2.50976500 & -1.25363300 \\
\hline C & 4.60310400 & 4.24651100 & -0.71741800 \\
\hline C & 5.13431300 & 4.21734500 & -2.16457700 \\
\hline $\mathrm{H}$ & 6.11076400 & 4.72420200 & -2.22553400 \\
\hline $\mathrm{H}$ & 4.43757800 & 4.73451900 & -2.84637200 \\
\hline $\mathrm{H}$ & 5.27739700 & 3.18894400 & -2.52539400 \\
\hline C & 1.96858800 & 2.61477600 & 2.05018000 \\
\hline $\mathrm{H}$ & 2.60638300 & 2.17046900 & 2.81934400 \\
\hline $\mathrm{H}$ & 0.90284300 & 2.36856700 & 2.09534000 \\
\hline C & 2.36349300 & 3.80293800 & 1.4194600 \\
\hline
\end{tabular}




$\begin{array}{lrrr}\text { H } & 3.34017500 & 4.20069900 & 1.70192800 \\ \mathrm{C} & 1.32980300 & 4.79483200 & 0.95306300 \\ \mathrm{H} & 1.73730600 & 5.56578500 & 0.28826700 \\ \mathrm{H} & 0.49554900 & 4.28681900 & 0.44940000 \\ \mathrm{H} & 0.92417500 & 5.30209700 & 1.84543000 \\ \mathrm{H} & 5.33701800 & 3.72645600 & -0.08077000 \\ \mathrm{C} & 4.48483900 & 5.70378100 & -0.25297100 \\ \mathrm{H} & 4.19075000 & 5.80279400 & 0.80175000 \\ \mathrm{H} & 5.45588800 & 6.21086600 & -0.36431400 \\ \mathrm{H} & 3.75214000 & 6.25710900 & -0.86517400 \\ \mathrm{C} & 8.40704800 & 3.06915100 & 1.10509800 \\ \mathrm{H} & 7.31489700 & 3.09114200 & 1.27087200 \\ \mathrm{H} & 8.63760100 & 3.97160100 & 0.50627300 \\ \mathrm{C} & 10.45725000 & 0.97196400 & -0.87125900 \\ \mathrm{H} & 10.76143700 & 1.80549000 & -1.53369000 \\ \mathrm{H} & 11.24730200 & 0.91921500 & -0.09682800 \\ \mathrm{C} & 10.41000200 & -0.34372700 & -1.66722300 \\ \mathrm{H} & 11.38078000 & -0.59444900 & -2.13076500 \\ \mathrm{H} & 10.12030900 & -1.19193800 & -1.02480700 \\ \mathrm{H} & 9.66102200 & -0.29765700 & -2.47503700 \\ \mathrm{C} & 9.13707600 & 3.12997900 & 2.45855700 \\ \mathrm{H} & 10.23457800 & 3.13639400 & 2.33719000 \\ \mathrm{H} & 8.87583400 & 4.03100400 & 3.04226000 \\ \mathrm{H} & 8.88816300 & 2.25651900 & 3.08521900 \\ \mathrm{Cl} & 7.07198300 & 1.28753300 & -1.53590400 \\ \mathrm{Al} & 8.77651900 & 1.47000700 & 0.01897300\end{array}$

\begin{tabular}{|c|c|c|c|}
\hline $\mathrm{Cl}$ & -7.46750000 & -2.53501100 & 1.45863300 \\
\hline Mg & -6.38060300 & -4.30050100 & 0.05128100 \\
\hline $\mathrm{Cl}$ & -8.62034200 & -0.70970600 & -1.22281500 \\
\hline Mg & -6.49756600 & -0.66239200 & 0.10725400 \\
\hline $\mathrm{Cl}$ & -4.25776900 & -4.25253200 & 1.38123200 \\
\hline $\mathrm{Cl}$ & -1.16525500 & -2.33116700 & 1.35969100 \\
\hline $\mathrm{Cl}$ & -4.37454400 & -0.61361600 & 1.43692000 \\
\hline $\mathrm{Cl}$ & -2.20112900 & -4.14453200 & -1.37743300 \\
\hline Mg & -0.07830300 & -4.09657200 & -0.04750000 \\
\hline $\mathrm{Cl}$ & -5.41052800 & -2.42684300 & -1.30037100 \\
\hline Mg & -3.28798700 & -2.37930600 & 0.02999200 \\
\hline $\mathrm{Cl}$ & -2.31799600 & -0.50586000 & -1.32159000 \\
\hline Mg & -0.19429400 & -0.45798400 & 0.00872400 \\
\hline $\mathrm{Cl}$ & 2.04446300 & -4.04852300 & 1.28251300 \\
\hline $\mathrm{Cl}$ & 5.13727100 & -2.12716200 & 1.26118800 \\
\hline $\mathrm{Cl}$ & 1.87839900 & -0.50039800 & 1.47793600 \\
\hline
\end{tabular}




\begin{tabular}{|c|c|c|c|}
\hline $\mathrm{Cl}$ & 4.10132300 & -3.94057400 & -1.47611100 \\
\hline Mg & 6.22404800 & -3.89263600 & -0.14620200 \\
\hline $\mathrm{Cl}$ & 0.89160100 & -2.22318100 & -1.39898600 \\
\hline Mg & 3.01441700 & -2.17542600 & -0.06920600 \\
\hline $\mathrm{Cl}$ & 3.86964100 & -0.17822400 & -1.3396910 \\
\hline $\mathrm{Mg}$ & 6.10647400 & -0.25391700 & -0.0905340 \\
\hline $\mathrm{Cl}$ & 8.23005500 & -0.20598500 & 395980 \\
\hline $\mathrm{Cl}$ & 7.19398600 & -2.01924300 & -1.4976280 \\
\hline $\mathrm{Ti}$ & 2.94222400 & 1.68886900 & 0.20573306 \\
\hline $\mathrm{Cl}$ & 4.93604800 & 1.32735900 & 1.52365100 \\
\hline $\mathrm{Cl}$ & 0.58835400 & 1.71346500 & -0.64881106 \\
\hline C & -4.84471300 & 3.30528800 & 0.46370906 \\
\hline C & -3.45262200 & 2.59131900 & 0.50354806 \\
\hline C & -4.60560700 & 4.58445000 & -0.38622506 \\
\hline $\mathrm{H}$ & -5.09727200 & 3.64174200 & 1.48651506 \\
\hline C & -2.44059900 & 3.60877300 & -0.0854490 \\
\hline $\mathrm{H}$ & -3.44609600 & 1.65555800 & -0.06815006 \\
\hline $\mathrm{H}$ & -3.20137300 & 2.30881100 & 1.53694806 \\
\hline C & -3.15846200 & 4.96406900 & -0.05747100 \\
\hline $\mathrm{H}$ & -5.33573600 & 5.38139600 & -0.1774760 \\
\hline $\mathrm{H}$ & -4.67737700 & 4.35838400 & -1.46604506 \\
\hline $\mathrm{H}$ & -1.47973600 & 3.61652700 & 0.44899200 \\
\hline $\mathrm{H}$ & -2.20462600 & 3.33697400 & -1.12737206 \\
\hline H & -3.11534700 & 5.40306300 & 0.95581106 \\
\hline $\mathrm{H}$ & -2.73100200 & 5.70198100 & $-0.7553810 e$ \\
\hline C & -7.61910500 & 3.40092900 & -0.8681340 \\
\hline C & -8.89733800 & 2.76463000 & -1.45544806 \\
\hline C & -8.19193000 & 4.36109000 & 0.21587706 \\
\hline $\mathrm{H}$ & -7.07695800 & 3.97935400 & -1.63737806 \\
\hline C & -9.80020400 & 2.52508100 & -0.2328950 \\
\hline $\mathrm{H}$ & -8.72611900 & 1.83287600 & -2.0120800 \\
\hline $\mathrm{H}$ & -9.36185500 & 3.49587400 & -2.1418080 \\
\hline C & -9.43488200 & 3.63307500 & 0.79148900 \\
\hline $\mathrm{H}$ & -8.49811600 & 5.30006200 & -0.27448606 \\
\hline $\mathrm{H}$ & -7.46519400 & 4.64812200 & 0.99490100 \\
\hline $\mathrm{H}$ & -10.86704800 & 2.53289700 & -0.50352200 \\
\hline $\mathrm{H}$ & -9.58175500 & 1.52512500 & 0.16900200 \\
\hline $\mathrm{H}$ & -10.25956800 & 4.34556900 & 0.95009000 \\
\hline $\mathrm{H}$ & -9.21661000 & 3.19351900 & 1.77756800 \\
\hline 0 & -5.83228700 & 0.98620400 & -1.11232000 \\
\hline 0 & -6.90298000 & 1.22887900 & 1.07519100 \\
\hline C & -6.95049000 & 1.43688600 & 2.48366000 \\
\hline $\mathrm{H}$ & -5.97444200 & 1.20064600 & $2.935477 €$ \\
\hline - & -7.23256800 & 2.47442200 & 2.7232170 \\
\hline
\end{tabular}




\begin{tabular}{|c|c|c|c|}
\hline H & -7.71079100 & 0.75987300 & 2.89870600 \\
\hline C & -5.73536900 & 0.91134000 & -2.53710800 \\
\hline $\mathrm{H}$ & -4.77394100 & 0.44172600 & -2.78976700 \\
\hline $\mathrm{H}$ & -6.57440900 & 0.30198200 & -2.90511800 \\
\hline $\mathrm{H}$ & -5.78064300 & 1.91470000 & -2.99022306 \\
\hline Si & -6.30670800 & 2.28052600 & -0.12920900 \\
\hline C & 3.35934900 & 3.18254300 & $-1.1384970 e$ \\
\hline $\mathrm{H}$ & 4.32156100 & 3.43590200 & -0.63745400 \\
\hline $\mathrm{H}$ & 3.62736300 & 2.63574300 & -2.06128800 \\
\hline C & 2.47896200 & 4.41081600 & -1.42624900 \\
\hline C & 1.84232200 & 4. 31180800 & -2.81728100 \\
\hline C & 3.26264700 & 5.71943700 & $-1.2553210 e$ \\
\hline $\mathrm{H}$ & 1.64296200 & 4.43051600 & -0.70191500 \\
\hline $\mathrm{H}$ & 1.15431900 & 5.15332700 & -3.00077100 \\
\hline $\mathrm{H}$ & 1.27153800 & 3.37676700 & -2.92129400 \\
\hline $\mathrm{H}$ & 2.61803000 & 4.32964600 & -3.60183000 \\
\hline $\mathrm{H}$ & 2.62072700 & 6.59711000 & -1.43955100 \\
\hline $\mathrm{H}$ & 4.10901900 & 5.76301700 & -1.96116600 \\
\hline $\mathrm{H}$ & 3.67562100 & 5.81052100 & -0.23663900 \\
\hline C & 1.89126700 & 2.62684000 & 2.62619300 \\
\hline $\mathrm{H}$ & 2.50081800 & 1.91693400 & 3.19961300 \\
\hline C & 2.52766000 & 3.61285700 & 1.95569200 \\
\hline $\mathrm{H}$ & 1.96551400 & 4.36527200 & 1.39500700 \\
\hline H & 3.60838300 & 3.75197700 & 2.04104100 \\
\hline C & 0.40811400 & 2.46444900 & 2.73447000 \\
\hline $\mathrm{H}$ & 0.11007700 & 1.41887100 & 2.56590000 \\
\hline $\mathrm{H}$ & 0.09462700 & 2.71686000 & 3.76248100 \\
\hline $\mathrm{H}$ & -0.13487500 & 3.10833100 & 2.03062200 \\
\hline C & 8.35469500 & 3.23165400 & 1.37368700 \\
\hline $\mathrm{H}$ & 7.25449700 & 3.24316700 & 1.47923600 \\
\hline $\mathrm{H}$ & 8.61760100 & 4.19441900 & 0.89449400 \\
\hline C & 10.45282300 & 1.42083700 & -0.83956200 \\
\hline $\mathrm{H}$ & 10.70206100 & 2.32083700 & -1.43430900 \\
\hline $\mathrm{H}$ & 11.26704800 & 1.33637800 & -0.09349100 \\
\hline C & 10.43561400 & 0.17750800 & -1.74554700 \\
\hline $\mathrm{H}$ & 11.40295200 & 0.00762900 & -2.25086600 \\
\hline $\mathrm{H}$ & 10.19579500 & -0.73441100 & -1.17366200 \\
\hline $\mathrm{H}$ & 9.66545100 & 0.26347200 & -2.52993700 \\
\hline C & 9.00844700 & 3.13764100 & 2.76388300 \\
\hline $\mathrm{H}$ & 10.11091900 & 3.15704700 & 2.70485700 \\
\hline $\mathrm{H}$ & 8.71065200 & 3.96809900 & 3.42887300 \\
\hline $\mathrm{H}$ & 8.73062400 & 2.19909300 & 3.27285500 \\
\hline $\mathrm{Cl}$ & 7.03997900 & 1.63155000 & -1.39358606 \\
\hline Al & 8.78013600 & 1.76439700 & 0.1337680 \\
\hline
\end{tabular}




\begin{tabular}{|c|c|c|c|}
\hline \multicolumn{4}{|c|}{$\mathrm{MgCl}_{2}-\mathrm{Ti}$-i sobutyl-DCPDMS-Mg2-AlEt ${ }_{2} \mathrm{Cl}$-Propylene-2,1-re-TS } \\
\hline $\mathrm{Cl}$ & -7.47898300 & -2.51422400 & 1.51893600 \\
\hline Mg & -6.40484800 & -4.29095000 & 0.11593200 \\
\hline $\mathrm{Cl}$ & -8.64734100 & -0.70488300 & -1.16660900 \\
\hline Mg & -6.51530400 & -0.65234900 & 0.14837200 \\
\hline $\mathrm{Cl}$ & -4.27275500 & -4.23776400 & 1.43078900 \\
\hline $\mathrm{Cl}$ & -1.17739400 & -2.32192600 & 1.37485200 \\
\hline $\mathrm{Cl}$ & -4.38302500 & -0.59836000 & 1.46293300 \\
\hline $\mathrm{Cl}$ & -2.23513400 & -4.15174100 & -1.34272300 \\
\hline Mg & -0.10307900 & -4.09856800 & -0.02791400 \\
\hline $\mathrm{Cl}$ & -5.44102700 & -2.42803800 & -1.25491400 \\
\hline Mg & -3.30925500 & -2.37523800 & 0.06039800 \\
\hline $\mathrm{Cl}$ & -2.34550600 & -0.51256700 & -1.31040400 \\
\hline Mg & -0.21203000 & -0.45969500 & 0.00481200 \\
\hline $\mathrm{Cl}$ & 2.02899600 & -4.04532200 & 1.28701500 \\
\hline $\mathrm{Cl}$ & 5.12476400 & -2.12948700 & 1.23136400 \\
\hline $\mathrm{Cl}$ & 1.85118000 & -0.53619000 & 1.53281100 \\
\hline $\mathrm{Cl}$ & 4.06681400 & -3.95931800 & -1.48644400 \\
\hline Mg & 6.19881100 & -3.90615100 & -0.17163100 \\
\hline $\mathrm{Cl}$ & 0.86058500 & -2.23592800 & -1.39857700 \\
\hline Mg & 2.99242000 & -2.18286200 & -0.08360200 \\
\hline $\mathrm{Cl}$ & 3.80764200 & -0.17807900 & -1.31281200 \\
\hline Mg & 6.08773500 & -0.26682200 & -0.13959400 \\
\hline $\mathrm{Cl}$ & 8.22052200 & -0.21378900 & 1.17554300 \\
\hline $\mathrm{Cl}$ & 7.16246100 & -2.04355100 & -1.54221100 \\
\hline $\mathrm{Ti}$ & 2.85369200 & 1.60753700 & 0.46565500 \\
\hline $\mathrm{Cl}$ & 5.02987300 & 1.34694300 & 1.50057900 \\
\hline $\mathrm{Cl}$ & 0.66410100 & 1.66200000 & -0.74953900 \\
\hline C & -4.87521000 & 3.34656800 & 0.33244800 \\
\hline C & -3.53905400 & 2.63960300 & 0.65855300 \\
\hline C & -4.45229200 & 4. 38199600 & -0.73873100 \\
\hline $\mathrm{H}$ & -5.21078000 & 3.91132900 & 1.22520400 \\
\hline C & -2.44879100 & 3.73846600 & 0.52299300 \\
\hline $\mathrm{H}$ & -3.36071900 & 1.82916600 & -0.06355700 \\
\hline $\mathrm{H}$ & -3.55010900 & 2.15890000 & 1.64614700 \\
\hline C & -3.12113400 & 4.93321300 & -0.20175700 \\
\hline $\mathrm{H}$ & -5.20319900 & 5.16861000 & -0.91148900 \\
\hline $\mathrm{H}$ & -4.28398000 & 3.87254500 & -1.70569600 \\
\hline $\mathrm{H}$ & -2.07037000 & 4.04917000 & 1.50938900 \\
\hline $\mathrm{H}$ & -1.58596000 & 3.35736400 & -0.04365300 \\
\hline $\mathrm{H}$ & -3.33293400 & 5.74433500 & 0.51486000 \\
\hline $\mathrm{H}$ & -2.49171600 & 5.36484600 & -0.99589300 \\
\hline C & -7.60893900 & 3.37072800 & -1.03957700 \\
\hline
\end{tabular}




\begin{tabular}{|c|c|c|c|}
\hline C & -8.90214300 & 2.72631600 & -1.58101600 \\
\hline C & -8.16643300 & 4.43951000 & -0.05830700 \\
\hline $\mathrm{H}$ & -7.04063900 & 3.85939900 & -1.85271100 \\
\hline C & -9.81849600 & 2.61449200 & -0.34809006 \\
\hline $\mathrm{H}$ & -8.75498900 & 1.74897500 & -2.05978906 \\
\hline $\mathrm{H}$ & -9.34112500 & 3.41415900 & -2.32626306 \\
\hline C & -9.39523100 & 3.76728300 & 0.60521200 \\
\hline H & -8.49102500 & 5.31482700 & -0.6459380 \\
\hline $\mathrm{H}$ & -7.43307400 & 4.81654300 & $0.6742380 e$ \\
\hline $\mathrm{H}$ & -10.88243700 & 2.66569400 & -0.6253880 \\
\hline $\mathrm{H}$ & -9.65856300 & 1.63196900 & 0.11976900 \\
\hline $\mathrm{H}$ & -10.20127100 & 4.50027600 & 0.7661410 \\
\hline $\mathrm{H}$ & -9.13817800 & 3.37278000 & 1.60153800 \\
\hline 0 & -5.83259900 & 0.95137300 & -1.13914206 \\
\hline 0 & -6.90548700 & 1.28013100 & 1.03749800 \\
\hline C & -6.94117200 & 1.54979600 & $2.4362610 e$ \\
\hline $\mathrm{H}$ & -5.97818600 & 1.27874700 & 2.89647100 \\
\hline $\mathrm{H}$ & -7.16267600 & 2.61097600 & 2.63257500 \\
\hline $\mathrm{H}$ & -7.73807800 & 0.93548600 & 2.87941400 \\
\hline C & -5.74747700 & 0.82093900 & -2.5608210 \\
\hline $\mathrm{H}$ & -4.79382600 & 0.33017000 & -2.80105706 \\
\hline $\mathrm{H}$ & -6.59636700 & 0.20923500 & -2.90143100 \\
\hline $\mathrm{H}$ & -5.78201700 & 1.80697700 & -3.0510780 \\
\hline Si & -6.32158600 & 2.28115700 & -0.21435600 \\
\hline C & 3.39323700 & 3.51480700 & -0.36099200 \\
\hline $\mathrm{H}$ & 4.35569400 & 3.96069400 & -0.07179200 \\
\hline $\mathrm{H}$ & 3.70659000 & 2.61994200 & -0.99084800 \\
\hline C & 2.53234400 & 4.45921400 & -1.20643300 \\
\hline C & 2.64644100 & 4.10839400 & -2.69914000 \\
\hline C & 2.90118000 & 5.92645400 & -0.95355700 \\
\hline $\mathrm{H}$ & 1.47691500 & 4.31003900 & -0.92081000 \\
\hline $\mathrm{H}$ & 2.02718500 & 4.78608300 & -3.30871300 \\
\hline $\mathrm{H}$ & 2.30564900 & 3.07834800 & -2.88680400 \\
\hline $\mathrm{H}$ & 3.68951800 & 4.19607800 & -3.04669300 \\
\hline $\mathrm{H}$ & 2.23587500 & 6.60121800 & -1.51523700 \\
\hline $\mathrm{H}$ & 3.93687100 & 6.12790600 & -1.27534200 \\
\hline $\mathrm{H}$ & 2.82710000 & 6.19593300 & 0.11228700 \\
\hline C & 2.02925700 & 2.57249000 & 2.34772200 \\
\hline $\mathrm{H}$ & 2.63713400 & 1.97049100 & 3.03598200 \\
\hline C & 2.65175500 & 3.68670500 & 1.78766600 \\
\hline $\mathrm{H}$ & 2.03944300 & 4.50925100 & 1.41289900 \\
\hline $\mathrm{H}$ & 3.67243500 & 3.93889200 & 2.07833000 \\
\hline C & 0.53047300 & 2.48247800 & 2.52821900 \\
\hline $\mathrm{H}$ & 0.17716900 & 1.44078900 & 2.5260740 \\
\hline
\end{tabular}




$\begin{array}{lrrr}\text { H } & 0.24512200 & 2.91285700 & 3.50408600 \\ \text { H } & -0.00568700 & 3.02865600 & 1.74314400 \\ \text { C } & 8.37319000 & 3.23133400 & 1.23889900 \\ \text { H } & 7.27257900 & 3.27642600 & 1.32749000 \\ \text { H } & 8.67416100 & 4.17689500 & 0.74772700 \\ \mathrm{C} & 10.43710300 & 1.36122300 & -0.94942900 \\ \text { H } & 10.68636700 & 2.24982800 & -1.56117900 \\ \text { H } & 11.25579400 & 1.28625500 & -0.20714600 \\ \mathrm{C} & 10.40952600 & 0.10260200 & -1.83361800 \\ \mathrm{H} & 11.37304200 & -0.08012900 & -2.34189600 \\ \mathrm{H} & 10.16939700 & -0.79812100 & -1.24443400 \\ \mathrm{H} & 9.63471100 & 0.17796000 & -2.61450800 \\ \mathrm{C} & 9.00113800 & 3.14136700 & 2.64120000 \\ \mathrm{H} & 10.10450700 & 3.12316300 & 2.60060800 \\ \mathrm{H} & 8.72042200 & 3.99317200 & 3.28647200 \\ \mathrm{H} & 8.68350500 & 2.22188600 & 3.16125500 \\ \mathrm{Cl} & 7.02337100 & 1.59004900 & -1.48868400 \\ \mathrm{Al} & 8.77322400 & 1.73012900 & 0.03054700\end{array}$

$\mathrm{MgCl}_{2}$-Ti-isobutyl-DCPDMS-Mg2-AlEt ${ }_{2} \mathrm{Cl}$-Propylene-2,1-si-coordination

$\begin{array}{lrrr}\text { Cl } & -7.53642000 & -2.49259700 & 1.41365400 \\ \text { Mg } & -6.43209200 & -4.25688200 & 0.01841200 \\ \text { Cl } & -8.66668500 & -0.67174000 & -1.28039700 \\ \text { Mg } & -6.55716300 & -0.61898400 & 0.07040200 \\ \text { Cl } & -4.32251700 & -4.20347300 & 1.36909200 \\ \text { Cl } & -1.23398200 & -2.27567500 & 1.37636700 \\ \text { Cl } & -4.44740000 & -0.56476600 & 1.42079100 \\ \text { Cl } & -2.23913100 & -4.09327300 & -1.36934200 \\ \text { Mg } & -0.12955400 & -4.03987000 & -0.01867300 \\ \text { Cl } & -5.45269400 & -2.38222900 & -1.32511300 \\ \text { Mg } & -3.34342800 & -2.32923800 & 0.02598800 \\ \text { Cl } & -2.36411200 & -0.45481200 & -1.31748100 \\ \text { Mg } & -0.25348200 & -0.40143900 & 0.03361900 \\ \text { Cl } & 1.97996200 & -3.98638800 & 1.33206500 \\ \text { Cl } & 5.06880000 & -2.05856400 & 1.33957000 \\ \text { Cl } & 1.81321700 & -0.41774000 & 1.50446400 \\ \text { Cl } & 4.06354000 & -3.87623900 & -1.40632800 \\ \text { Mg } & 6.17303500 & -3.82284600 & -0.05568800 \\ \text { Cl } & 0.84964400 & -2.16551900 & -1.36203900 \\ \text { Mg } & 2.95922200 & -2.11234700 & -0.01163500 \\ \text { Cl } & 3.82680400 & -0.14731300 & -1.29548700 \\ \text { Mg } & 6.04719300 & -0.18433400 & -0.00399300 \\ \text { Cl } & 8.15763100 & -0.13094900 & 1.34685600 \\ \text { Cl } & 7.15226400 & -1.94848000 & -1.39898400\end{array}$




\begin{tabular}{|c|c|c|c|}
\hline $\mathrm{Ti}$ & 2.88890900 & 1.76450100 & 0.15958100 \\
\hline $\mathrm{Cl}$ & 4.87138500 & 1.48446400 & 1.50718400 \\
\hline $\mathrm{Cl}$ & 0.49700300 & 1.80289300 & -0.60442500 \\
\hline C & -4.85577700 & 3.28843800 & 0.64079500 \\
\hline C & -3.46508400 & 2.58636200 & 0.71105400 \\
\hline C & -4.56225600 & 4.63207200 & -0.07623900 \\
\hline $\mathrm{H}$ & -5.16742600 & 3.54421900 & 1.67162500 \\
\hline C & -2.40912700 & 3.70786000 & 0.49174300 \\
\hline $\mathrm{H}$ & -3.37068200 & 1.80265700 & -0.05213300 \\
\hline $\mathrm{H}$ & -3.34180300 & 2.07155400 & 1.67347200 \\
\hline C & -3.19441800 & 5.03072200 & 0.48964000 \\
\hline $\mathrm{H}$ & -5.34545800 & 5.38898900 & 0.08563400 \\
\hline $\mathrm{H}$ & -4.47708600 & 4.48214000 & -1.16874000 \\
\hline $\mathrm{H}$ & -1.61375000 & 3.69301200 & 1.25402900 \\
\hline $\mathrm{H}$ & -1.90740500 & 3.56512200 & -0.47797400 \\
\hline $\mathrm{H}$ & -3.32659500 & 5.40275000 & 1.52118600 \\
\hline $\mathrm{H}$ & -2.70032200 & 5.83029700 & -0.08547800 \\
\hline C & -7.55585000 & 3.48118000 & -0.84142400 \\
\hline C & -8.79435000 & 2.83569300 & -1.50809400 \\
\hline C & -8.14845500 & 4.38524600 & 0.29776200 \\
\hline $\mathrm{H}$ & -6.99468500 & 4.09871900 & -1.56365000 \\
\hline C & -9.76416300 & 2.62806000 & -0.34073300 \\
\hline $\mathrm{H}$ & -8.58710500 & 1.89287200 & -2.03275300 \\
\hline $\mathrm{H}$ & -9.22289400 & 3.54795800 & -2.23670400 \\
\hline C & -9.61611700 & 3.91020300 & 0.49826000 \\
\hline $\mathrm{H}$ & -8.11174100 & 5.44667500 & 0.00707000 \\
\hline $\mathrm{H}$ & -7.57227800 & 4.32295900 & 1.23742400 \\
\hline $\mathrm{H}$ & -10.79804800 & 2.44760700 & -0.67264000 \\
\hline $\mathrm{H}$ & -9.45126300 & 1.73435200 & 0.22136900 \\
\hline $\mathrm{H}$ & -10.30665400 & 4.68476800 & 0.12624200 \\
\hline $\mathrm{H}$ & -9.86299000 & 3.75336600 & 1.56064200 \\
\hline 0 & -5.82509400 & 1.04047500 & -1.09872900 \\
\hline 0 & -6.98166000 & 1.24655300 & 1.04484400 \\
\hline C & -7.13836700 & 1.40549200 & 2.44977100 \\
\hline $\mathrm{H}$ & -6.15722500 & 1.47555800 & 2.94604900 \\
\hline $\mathrm{H}$ & -7.74443400 & 2.29693400 & 2.67863200 \\
\hline $\mathrm{H}$ & -7.66005000 & 0.51555900 & 2.82942600 \\
\hline C & -5.71612500 & 0.99476700 & -2.52412400 \\
\hline $\mathrm{H}$ & -4.75037000 & 0.53578700 & -2.77920900 \\
\hline $\mathrm{H}$ & -6.54879000 & 0.38787700 & -2.91063100 \\
\hline $\mathrm{H}$ & -5.76572900 & 2.00642800 & -2.95778500 \\
\hline Si & -6.29227000 & 2.31910800 & -0.09170700 \\
\hline C & 3.47547400 & 3.16121900 & -1.22850300 \\
\hline $\mathrm{H}$ & 3.51058100 & 4.04308700 & -0.55221200 \\
\hline
\end{tabular}




\begin{tabular}{lrrr} 
H & 4.52911400 & 2.85417200 & -1.39178900 \\
$\mathrm{C}$ & 2.77438200 & 3.50351400 & -2.54700600 \\
$\mathrm{C}$ & 2.72229900 & 2.30138700 & -3.49669300 \\
$\mathrm{C}$ & 3.45425700 & 4.70577000 & -3.22273300 \\
$\mathrm{H}$ & 1.73459200 & 3.79013900 & -2.30737100 \\
$\mathrm{H}$ & 2.24519500 & 2.57585200 & -4.45130600 \\
$\mathrm{H}$ & 2.15114900 & 1.46992100 & -3.05906500 \\
$\mathrm{H}$ & 3.73806200 & 1.93227100 & -3.71720700 \\
$\mathrm{H}$ & 2.93091600 & 4.98740600 & -4.15185600 \\
$\mathrm{H}$ & 4.49911500 & 4.46460600 & -3.48193800 \\
$\mathrm{H}$ & 3.46871000 & 5.58694400 & -2.56058400 \\
$\mathrm{C}$ & 1.69865600 & 2.71800700 & 2.63658900 \\
$\mathrm{C}$ & 2.16655700 & 3.68439000 & 1.81974100 \\
$\mathrm{H}$ & 1.54318500 & 4.07670900 & 1.00998800 \\
$\mathrm{H}$ & 3.12967400 & 4.16979600 & 2.00761500 \\
$\mathrm{H}$ & 0.71018500 & 2.29745800 & 2.41395800 \\
$\mathrm{C}$ & 2.39319300 & 2.18894700 & 3.84854700 \\
$\mathrm{H}$ & 1.80067900 & 2.44298100 & 4.74500500 \\
$\mathrm{H}$ & 2.45817300 & 1.09089900 & 3.80823800 \\
$\mathrm{H}$ & 3.40613000 & 2.60029800 & 3.95672300 \\
$\mathrm{C}$ & 8.33235000 & 3.30476100 & 1.36040200 \\
$\mathrm{H}$ & 7.22996000 & 3.34725700 & 1.42763300 \\
$\mathrm{H}$ & 8.63648000 & 4.24494800 & 0.86125400 \\
$\mathrm{C}$ & 10.45252300 & 1.38852000 & -0.73849900 \\
$\mathrm{H}$ & 10.73151700 & 2.26661400 & -1.35252300 \\
$\mathrm{H}$ & 11.24601700 & 1.31345000 & 0.03057400 \\
$\mathrm{C}$ & 10.43877000 & 0.11963500 & -1.60835200 \\
$\mathrm{H}$ & 11.41518200 & -0.07780300 & -2.08547900 \\
$\mathrm{H}$ & 10.17296100 & -0.77185500 & -1.01611600 \\
$\mathrm{H}$ & 9.68799900 & 0.19267600 & -2.41250500 \\
$\mathrm{C}$ & 8.93683600 & 3.23796700 & 2.77425500 \\
$\mathrm{H}$ & 10.04079700 & 3.22739800 & 2.75236900 \\
$\mathrm{H}$ & 8.63923000 & 4.09638500 & 3.40292400 \\
\hline & 8.61767000 & 2.32321000 & 3.30189500 \\
\hline & 8.75765400 & 1.62225100 & -1.38177200 \\
$\mathrm{H}$ & & 1.78898500 & 0.18194600
\end{tabular}

$\mathrm{MgCl}_{2}$-Ti-isobutyl-DCPDMS-Mg2-AlEt ${ }_{2} \mathrm{Cl}$-Propylene-2,1-si-TS

$\begin{array}{lllr}\text { Cl } & -7.56977000 & -2.45032600 & 1.48039800 \\ \text { Mg } & -6.50447800 & -4.23419500 & 0.07971900 \\ \text { Cl } & -8.70836100 & -0.62605800 & -1.20783600 \\ \text { Mg } & -6.58260100 & -0.59481600 & 0.11794500 \\ \text { Cl } & -4.37865600 & -4.20230100 & 1.40537700 \\ \text { Cl } & -1.26566500 & -2.31415700 & 1.36858800\end{array}$




\begin{tabular}{|c|c|c|c|}
\hline $\mathrm{Cl}$ & -4.45658700 & -0.56211600 & 1.44330600 \\
\hline $\mathrm{Cl}$ & -2.32638100 & -4.12978700 & -1.35769500 \\
\hline Mg & -0.20057000 & -4.09788100 & -0.03203500 \\
\hline $\mathrm{Cl}$ & -5.51715700 & -2.37764900 & -1.28301200 \\
\hline Mg & -3.39163600 & -2.34618200 & 0.04305800 \\
\hline $\mathrm{Cl}$ & -2.40440500 & -0.48982400 & -1.31960800 \\
\hline Mg & -0.27820300 & -0.45792600 & 0.00621600 \\
\hline $\mathrm{Cl}$ & 1.92522100 & -4.06594700 & 1.29365400 \\
\hline $\mathrm{Cl}$ & 5.03821100 & -2.17776700 & 1.25688800 \\
\hline $\mathrm{Cl}$ & 1.75849700 & -0.49786900 & 1.51706500 \\
\hline $\mathrm{Cl}$ & 3.97766100 & -3.99347700 & -1.46936300 \\
\hline Mg & 6.10341800 & -3.96157700 & -0.14375800 \\
\hline $\mathrm{Cl}$ & 0.78660400 & -2.24163300 & -1.39462300 \\
\hline Mg & 2.91238800 & -2.20990600 & -0.06917100 \\
\hline $\mathrm{Cl}$ & 3.76829100 & -0.24264600 & -1.32206900 \\
\hline Mg & 6.02491400 & -0.32169800 & -0.10563900 \\
\hline $\mathrm{Cl}$ & 8.15118300 & -0.28971300 & 1.21999100 \\
\hline $\mathrm{Cl}$ & 7.09051000 & -2.10532600 & -1.50628600 \\
\hline $\mathrm{Ti}$ & 2.80753300 & 1.61537700 & 0.37953200 \\
\hline $\mathrm{Cl}$ & 4.92075900 & 1.27165500 & 1.52776200 \\
\hline $\mathrm{Cl}$ & 0.66995800 & 1.59073600 & -0.91203500 \\
\hline C & -4.76444000 & 3.31582800 & 0.47927800 \\
\hline C & -3.43147500 & 2.55195100 & 0.71685300 \\
\hline C & -4.34264500 & 4.49189700 & -0.43784400 \\
\hline $\mathrm{H}$ & -5.09159600 & 3.76159900 & 1.43929800 \\
\hline C & -2.29338800 & 3.59426000 & 0.50415200 \\
\hline $\mathrm{H}$ & -3.33119600 & 1.72311200 & 0.00381500 \\
\hline $\mathrm{H}$ & -3.40891000 & 2.09049400 & 1.71305900 \\
\hline C & -2.99389000 & 4.92328000 & 0.15257000 \\
\hline $\mathrm{H}$ & -5.08174700 & 5.30744800 & -0.47248500 \\
\hline $\mathrm{H}$ & -4.19787900 & 4.13779300 & -1.47568200 \\
\hline $\mathrm{H}$ & -1.64757900 & 3.69561500 & 1.39194700 \\
\hline $\mathrm{H}$ & -1.63900300 & 3.27430000 & -0.32147800 \\
\hline $\mathrm{H}$ & -3.17840200 & 5.51395200 & 1.06681700 \\
\hline $\mathrm{H}$ & -2.40567000 & 5.55740200 & -0.53004700 \\
\hline C & -7.46799700 & 3.49491500 & -0.98101200 \\
\hline C & -8.75469300 & 2.87957400 & -1.57327900 \\
\hline C & -8.03084800 & 4.54058200 & 0.02240200 \\
\hline $\mathrm{H}$ & -6.87133300 & 3.99913100 & -1.76331000 \\
\hline C & -9.70261900 & 2.74843900 & -0.36762400 \\
\hline $\mathrm{H}$ & -8.60899900 & 1.91073200 & -2.07092700 \\
\hline $\mathrm{H}$ & -9.16701000 & 3.58789000 & -2.31462000 \\
\hline C & -9.31088800 & 3.89033700 & 0.61005700 \\
\hline $\mathrm{H}$ & -8.29582600 & 5.45418000 & -0.53567200 \\
\hline & & & S142 \\
\hline
\end{tabular}




\begin{tabular}{|c|c|c|c|}
\hline $\mathrm{H}$ & -7.31356000 & 4.85442200 & 0.79949300 \\
\hline $\mathrm{H}$ & -10.75932800 & 2.79528100 & -0.67170100 \\
\hline $\mathrm{H}$ & -9.54847400 & 1.76078200 & 0.09108500 \\
\hline $\mathrm{H}$ & -10.10967700 & 4.64035500 & 0.72066900 \\
\hline $\mathrm{H}$ & -9.12506200 & 3.49137300 & 1.61998700 \\
\hline 0 & -5.82229800 & 1.00813400 & -1.11696300 \\
\hline 0 & -6.92578700 & 1.33797400 & 1.04288900 \\
\hline C & -6.99296900 & 1.56559300 & 2.44760500 \\
\hline $\mathrm{H}$ & -6.03687100 & 1.29433000 & 2.92190200 \\
\hline $\mathrm{H}$ & -7.23623400 & 2.61688700 & 2.66994600 \\
\hline $\mathrm{H}$ & -7.78928500 & 0.92613100 & 2.85463100 \\
\hline C & -5.72877900 & 0.91055500 & -2.54098506 \\
\hline $\mathrm{H}$ & -4.78083200 & 0.41245000 & -2.78824100 \\
\hline $\mathrm{H}$ & -6.58388900 & 0.31848400 & -2.90019900 \\
\hline $\mathrm{H}$ & -5.74891400 & 1.90805200 & -3.00828900 \\
\hline Si & -6.24087300 & 2.33868700 & -0.15812400 \\
\hline C & 3.52452800 & 3.45142200 & -0.48248800 \\
\hline $\mathrm{H}$ & 4.42451000 & 3.88366500 & -0.01966000 \\
\hline $\mathrm{H}$ & 3.89518400 & 2.48853400 & -0.95264600 \\
\hline C & 2.95980700 & 4.32317800 & -1.60192000 \\
\hline C & 3.69794400 & 4.00531700 & -2.91573100 \\
\hline C & 3.05252000 & 5.81865700 & -1.27494800 \\
\hline $\mathrm{H}$ & 1.89903300 & 4.05255400 & -1.74848400 \\
\hline $\mathrm{H}$ & 3.32226000 & 4.63640600 & -3.73730300 \\
\hline $\mathrm{H}$ & 3.55751500 & 2.95176100 & -3.20543000 \\
\hline $\mathrm{H}$ & 4.78131500 & 4.18359900 & -2.81348900 \\
\hline $\mathrm{H}$ & 2.63761800 & 6.42250400 & -2.09727800 \\
\hline $\mathrm{H}$ & 4.10480900 & 6.11964100 & -1.13528900 \\
\hline $\mathrm{H}$ & 2.50311600 & 6.08672100 & -0.35985800 \\
\hline C & 1.78972300 & 2.73891000 & 2.03878600 \\
\hline C & 2.14192600 & 3.82654100 & 1.23294200 \\
\hline $\mathrm{H}$ & 1.40696900 & 4.22255900 & 0.52987100 \\
\hline $\mathrm{H}$ & 2.89806400 & 4.53291100 & 1.58225700 \\
\hline $\mathrm{H}$ & 0.77975000 & 2.33995900 & 1.88714500 \\
\hline C & 2.42093700 & 2.46983400 & 3.38758900 \\
\hline $\mathrm{H}$ & 1.81489300 & 2.93844600 & 4.18330300 \\
\hline $\mathrm{H}$ & 2.47689500 & 1.39325200 & 3.60323300 \\
\hline $\mathrm{H}$ & 3.44012700 & 2.87746500 & 3.44550500 \\
\hline C & 8.29381300 & 3.15495300 & 1.32172500 \\
\hline $\mathrm{H}$ & 7.19385500 & 3.17823500 & 1.42506400 \\
\hline $\mathrm{H}$ & 8.57028500 & 4.10915300 & 0.83279500 \\
\hline C & 10.37132100 & 1.31080800 & -0.88058200 \\
\hline $\mathrm{H}$ & 10.62183300 & 2.20539400 & -1.48299800 \\
\hline $\mathrm{H}$ & 11.18845400 & 1.22827600 & -0.13743200 \\
\hline & & & S143 \\
\hline
\end{tabular}




$\begin{array}{lrrr}\text { C } & 10.34511000 & 0.06088900 & -1.77717800 \\ \text { H } & 11.30967700 & -0.11706700 & -2.28505500 \\ \text { H } & 10.10349300 & -0.84555400 & -1.19745800 \\ \text { H } & 9.57214900 & 0.14431000 & -2.55910100 \\ \text { C } & 8.94218200 & 3.06749300 & 2.71487300 \\ \text { H } & 10.04504200 & 3.07095500 & 2.65966800 \\ \text { H } & 8.65352200 & 3.90915700 & 3.36978100 \\ \text { H } & 8.64901400 & 2.13866700 & 3.23278300 \\ \text { Cl } & 6.96244200 & 1.54809600 & -1.43581300 \\ \text { Al } & 8.70532100 & 1.66925300 & 0.09919400\end{array}$

